\title{
Optimizing Rehabilitation in Single-Sided Deafness
}

Restoration of binaural hearing by cochlear implantation

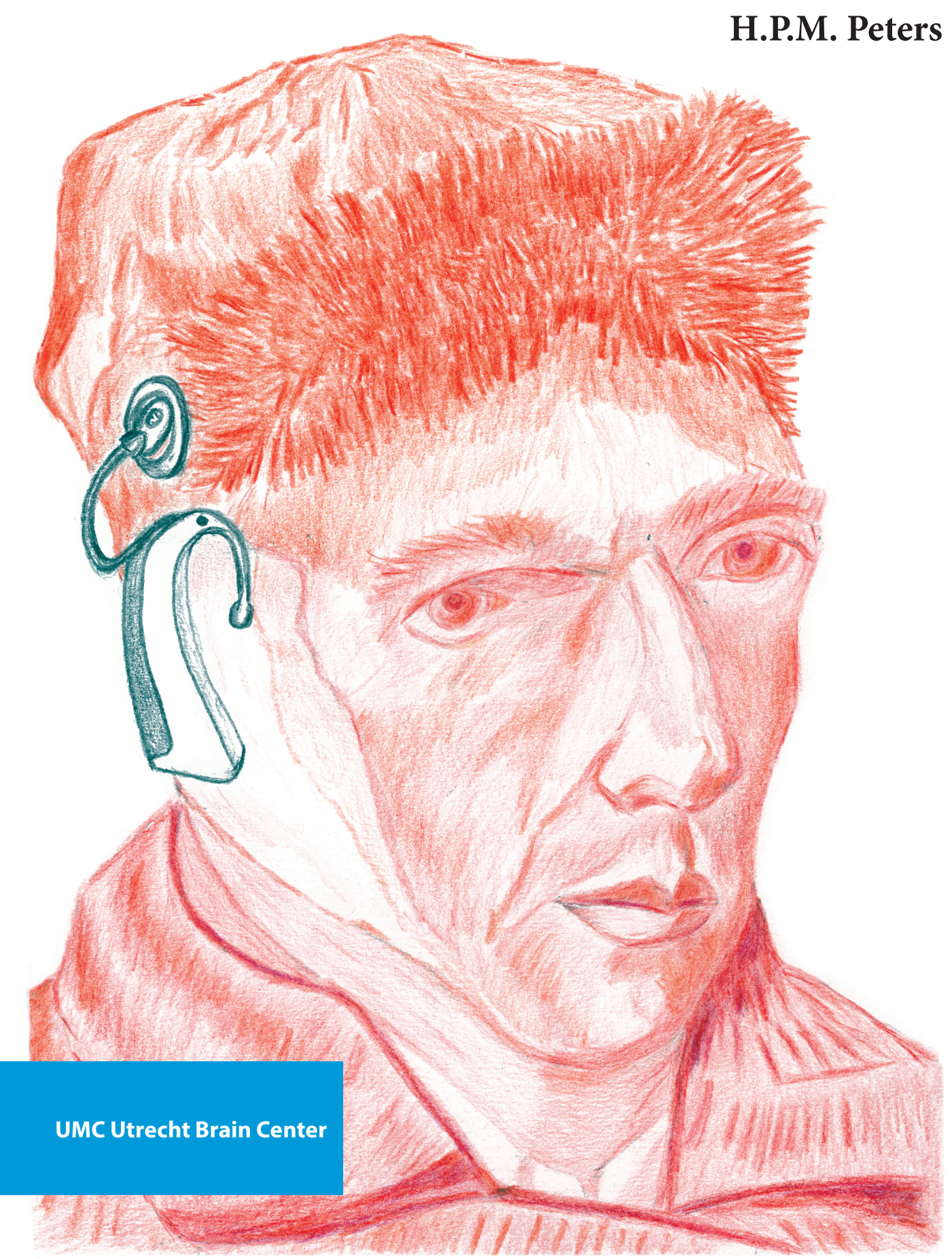





\section{Optimizing Rehabilitation in Single-Sided Deafness}

\section{Restoration of binaural hearing by cochlear implantation}

H.P.M. (Jeroen) Peters 


\section{COLOFON}

Cover: The cover is a drawing of Vincent van Gogh, famous for his paintings, but also for cutting off one of his ears (and thus having only one ear - although he probably heard with two ears). In this artist impression, he was rehabilitated with a cochlear implant. The colors of the drawing are red and white, after the city colors of Utrecht.

Idea: Jeroen Peters. Artist: Ten van Riel.

Design/lay-out: Wendy Bour-van Telgen, GildePrint, Enschede

Print: GildePrint, Enschede

ISBN: 978-94-6402-369-5

The research described in this thesis was performed at the University Medical Center Utrecht, Utrecht, the Netherlands. The research was partly funded by an unrestricted research grant from Cochlear Ltd. to the Department of Otorhinolaryngology and Head \& Neck Surgery.

Printing of this thesis was kindly supported by:

Amplifon, ALK, Allergy Therapeutics, Chipsoft, Cochlear, EmiD audiologische apparatuur, Makker hoortoestellen, MED-EL, MediTop, Mylan Healthcare B.V., Oticon Medical, Phonak, Stichting ORLU.

(C) H.P.M. (Jeroen) Peters, 2020.

All rights are reserved. No part of this book may be reproduced, distributed, stored in a retrieval system, or transmitted in any form or by any means, without prior written permission of the author. 


\title{
Optimizing Rehabilitation in Single-Sided Deafness \\ Restoration of binaural hearing by cochlear implantation
}

\author{
Optimaliseren van de behandeling van eenzijdige doofheid \\ Herstellen van binauraal horen door cochleaire implantatie \\ (met een samenvatting in het Nederlands)
}

\begin{abstract}
Proefschrift
ter verkrijging van de graad van doctor aan de Universiteit Utrecht op gezag van de rector magnificus, prof. dr. H.R.B.M. Kummeling, ingevolge het besluit van het college voor promoties in het openbaar te verdedigen op donderdag 3 december 2020 des middags te 2.30 uur

door
\end{abstract}

Hieronymus Petrus Maria Peters

geboren op

4 december 1986 te Houten 
Promotor:

Copromotoren:

Dr. A.L. Smit

Dr. G.A. van Zanten 
It never gets easier, you just go faster

Greg Lemond 


\section{TABLE OF CONTENTS}

\section{Part I. Introduction and literature reviews}

Chapter 1 Introduction and outline of thesis

Chapter 2 Review: bone conduction devices and contralateral routing of sound systems in single-sided deafness Laryngoscope 2015;125:218-226

Chapter 3 Cochlear implantation for patients with single-sided deafness or asymmetrical hearing loss: a systematic review of the evidence Otology \& Neurotology 2015;36:209-219

\section{Part II. CINGLE-trial}

Chapter 4 Study protocol CINGLE-trial: Cochlear Implantation for siNGLE-sided deafness, a randomised controlled trial and economic evaluation BMC Ear, Nose and Throat Disorders 2015;15:3

Chapter 5 Short-term outcomes of cochlear implantation for single-sided deafness compared to bone conduction devices and contralateral routing of sound hearing aids - results of a randomised controlled trial (CINGLE-trial) Submitted

\section{Part III. Vocoder and pitch match experiments}

Chapter 6 The sound of a cochlear implant investigated in patients with single-sided deafness and a cochlear implant Otology \& Neurotology 2018;39(6):707-714 
Chapter 7 Electro-acoustic pitch matching experiments in patients with single-sided deafness and a cochlear implant; is there a need for adjustment of the default frequency allocation tables? Hearing Research 2016;342:124-133

Chapter 8 Comparison of place-versus-pitch mismatch between a perimodiolar and lateral wall cochlear implant electrode array in patients with single-sided deafness and a cochlear implant Audiology \& Neurotology 2019;24(1):38-48

\section{Part IV. Quality of reporting}

Chapter 9 Reporting quality of systematic reviews and meta-analyses of otorhinolaryngologic articles PLoS One 2015;10(8):e0136540

Chapter 10 Assessment of the quality of reporting of randomised controlled trials in otorhinolaryngologic literature

PLoS One 2015;10(3):e0122328

\section{Part V. Discussion}

Chapter 11 Summary and General Discussion

\section{Appendices}

List of Abbreviations

List of Publications

PhD Portfolio

Acknowledgements | Dankwoord

About the Author | Curriculum Vitae 


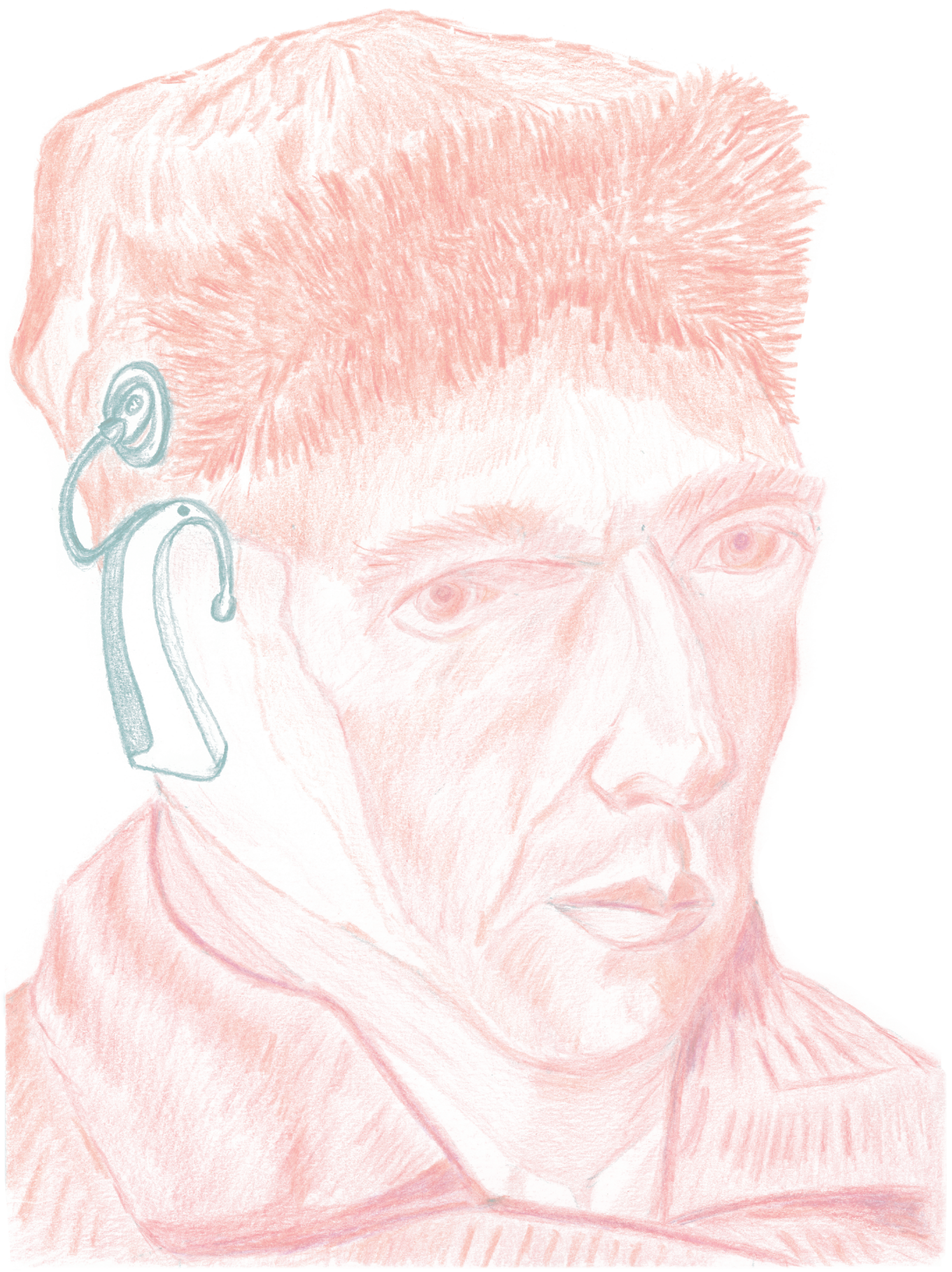




\section{Part I}

Introduction and Literature Reviews 


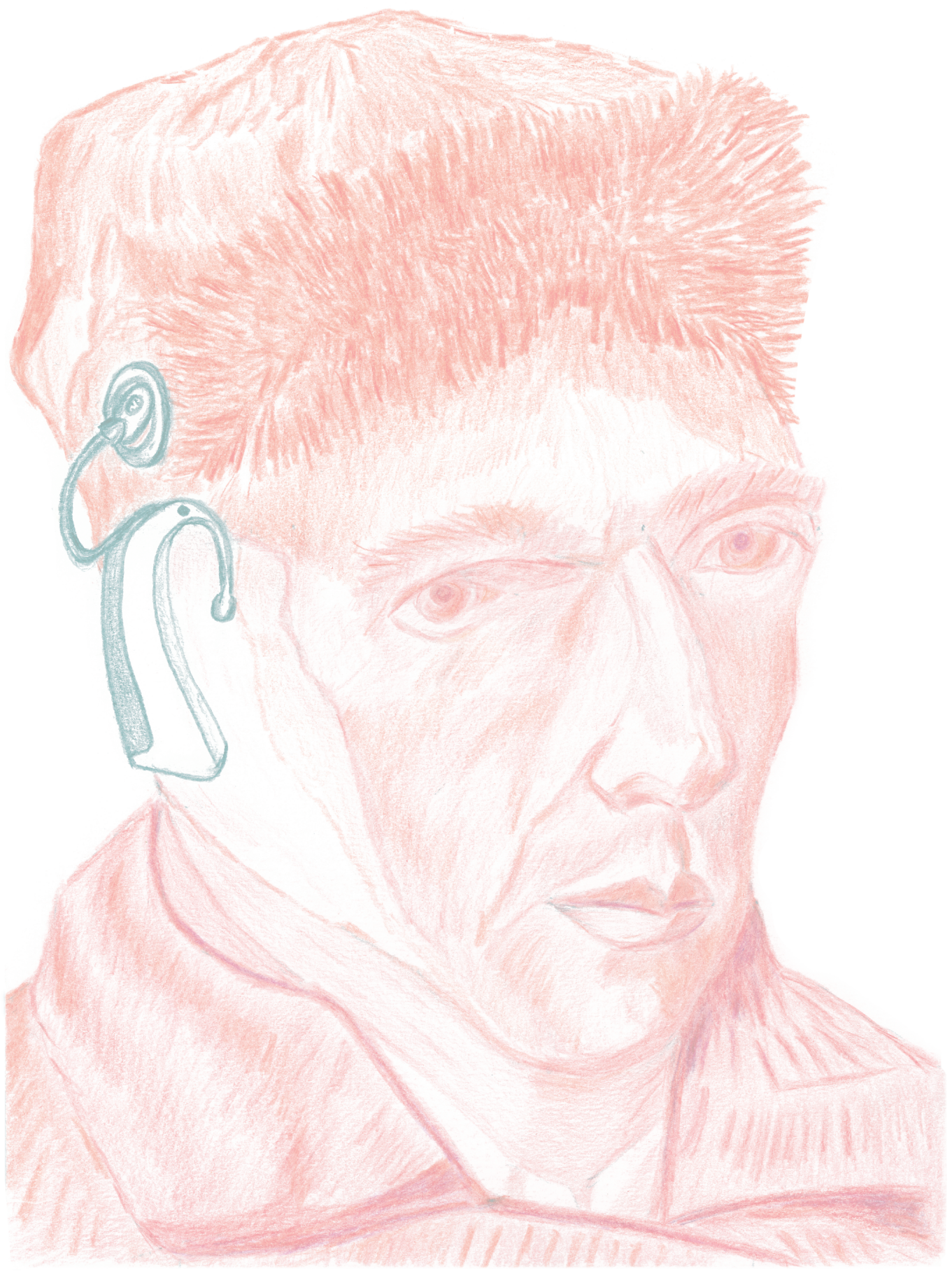




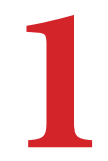

Introduction and outline of thesis 


\section{INTRODUCTION AND OUTLINE OF THESIS}

\subsection{Physiology of hearing}

Sound is vibration of air pressure. Humans have the amazing ability to hear sounds with a vibration frequency of $20 \mathrm{~Hz}$ up to $20,000 \mathrm{~Hz}$. Moreover, the dynamic range of the human ear enables us to hear sounds with the loudness of a falling leaf ( $20 \mathrm{~dB})$ as well as airplanes during take-off $(120 \mathrm{~dB})$. Because the decibel scale is logarithmic, the latter sound carries 50,000 times the energy of the first.

In Figure 1 the human ear is depicted. Sound travels through the external acoustic meatus and then reaches the tympanic membrane. The tympanic membrane transfers the sound from the external acoustic meatus to the three ossicles (from lateral to medial: malleus, incus and stapes) in the middle ear. At the stapes footplate, the sound vibrations are transferred to the fluid in the cochlea (perilymph), with only minimal loss of energy.

Figure 1: The human ear and its components.

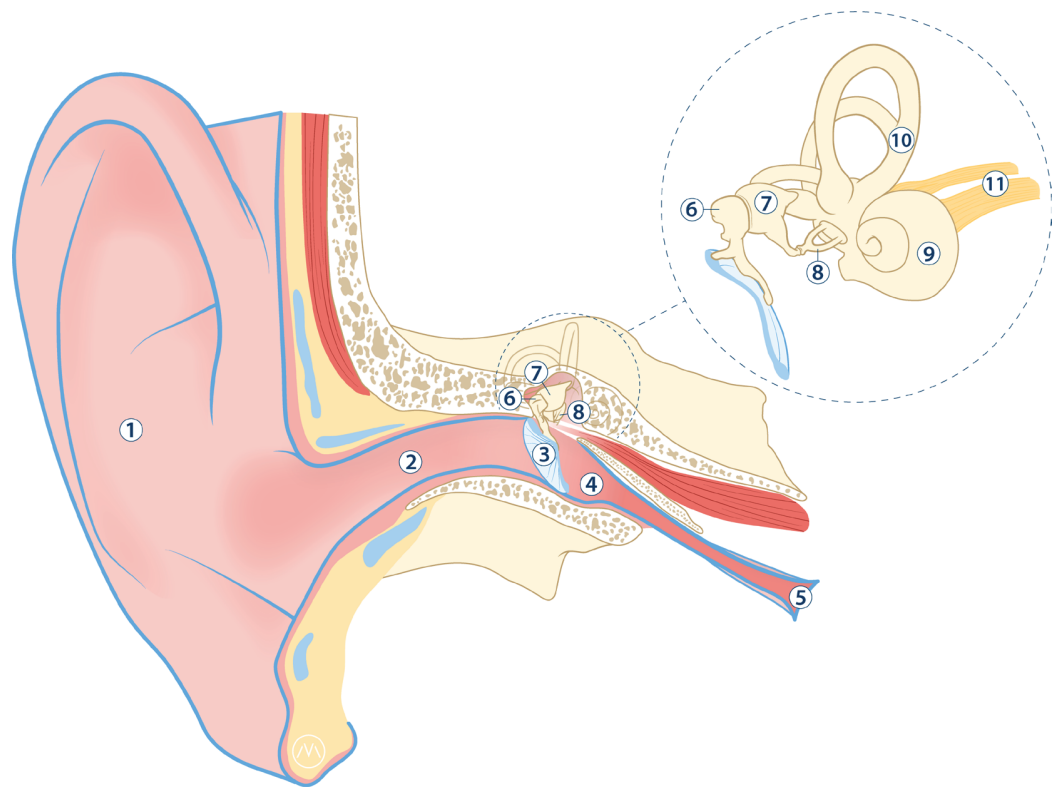

1. pinna, 2. external acoustic meatus, 3. tympanic membrane, 4. middle ear, 5. Eustachian tube, 6. malleus, 7. incus, 8. stapes, 9. cochlea, 10. semicircular canals, 11. vestibulocochlear nerve. 
The cochlea is a spiraling duct, split by two membranes into three parallel compartments (scala vestibuli, scala media and scala tympani) (Figure 2). The fluid pressure leads to vibration of these membranes. The sensory epithelium is located on the basilar membrane. It consists of an assembly of hair cells and the tectorial membrane, that lies on the hair bundles on top of these hair cells (together referred to as the organ of Corti). With vibration of the basilar membrane, the hair bundles vibrate relative to the surface of the hair cells, thereby admitting electrical current into the hair cells. This electrical current leads to the generation of action potentials in the dendrites of the auditory nerve cells (spiral ganglion in Figure 2). Ultimately, these action potentials travel to the brain via the auditory nerve. In the brain the sound information is processed in the auditory cortex.

Figure 2: Left: a cross section of the cochlea. Right: the organ of Corti.

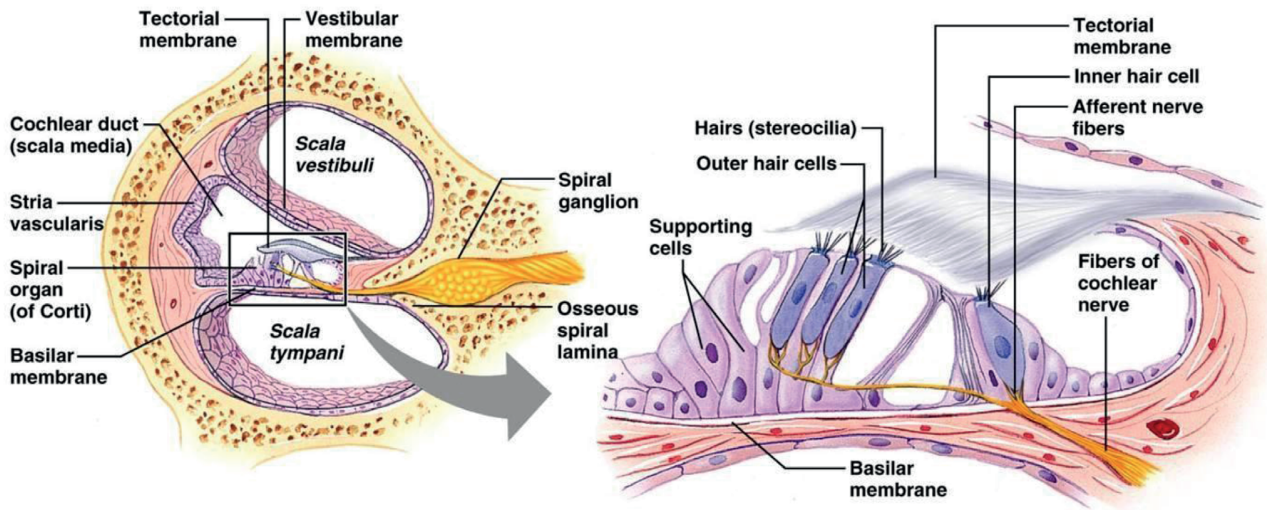

An important property of the cochlea is its tonotopic organization: different vibration frequencies lead to basilar membrane vibrations at different positions along the length of the cochlea: low frequency sounds lead to activated neurons in the apical part of the cochlea, whereas high frequency sounds lead to activated neurons in the basal part of the cochlea.

In the human skull, a cochlea is present in the mastoid bone on both sides which enables us to hear with two ears. 


\subsection{Binaural hearing}

Humans listen with two ears ("binaural hearing"). The human brain can determine from which direction a sound comes utilizing interaural time differences (ITD, mainly for sounds with a frequency lower than $1500 \mathrm{~Hz}$ ) and interaural level differences (ILD, mainly for sounds with a frequency higher than $1500 \mathrm{~Hz}$ ). Together, ITD and ILD lead to three effects that come into play with binaural hearing:

1) Squelch effect: the ability of the brain to separate sound and noise signals from spatially separated sources. ${ }^{1,2,3}$ When speech and noise come from different directions, the brain is able to separate the speech from the noise by comparing time, intensity and spectral differences between the two ears. The effect is that the brain is able to suppress signals that the listener does not wish to hear. ${ }^{4}$ In normal hearing subjects, the squelch effect provides a gain of $2-4.9 \mathrm{~dB}$ in the speech reception threshold in background noise. ${ }^{5-7}$

2) Summation effect: when identical signals are presented bilaterally, there is an advantage when hearing with two ears instead of hearing with just one ear (redundancy of auditory input)., ${ }^{2,8}$ The hearing threshold level is improved by $2-6 \mathrm{~dB}$ in the binaural listening condition over the monaural listening condition. ${ }^{9}$

3) Head shadow effect: the head acts as an acoustic barrier for the unwanted sounds, which results in a better signal-to-noise ratio.,10 When one ear is closer to the noise source, adding a second ear contralateral to the noise provides an ear with a better signal-to-noise ratio. The head shadow effect is more pronounced at high frequencies, towards as much as 10-16 dB level difference at frequencies above 1 $\mathrm{kHz} .^{11,12}$ The head shadow effect is not a true binaural effect (i.e. patients with one deaf ear can also benefit from the head shadow effect), but merely a geometric effect and does not require binaural processing by the brain. 


\subsection{Single-sided deafness (SSD)}

Hearing loss can occur due to various reasons. Possible etiologies of postlingual single-sided deafness (SSD) include a (viral) infection, idiopathic sudden deafness, Ménière's disease or labyrinthitis. SSD has been estimated to affect $12-27$ per 100.000 adults in the general population. ${ }^{3}$ When the hearing loss is caused by damaged hair cells, we refer to this type of hearing loss as cochlear hearing loss. In the remainder of this thesis, when we refer to singlesided deafness, we mean unilateral cochlear hearing loss. The onset of SSD was postlingual in all patients: this means that their auditory infrastructure developed normally, and all patients heard normally in both ears in the past.

Patients with SSD cannot benefit from the squelch effect and summation effect. They complain about difficulties with communication in noisy environments and a decreased ability of localizing sounds. Consequently, they perform worse than bilateral hearing subjects on speech perception in noise and on localization of sounds. ${ }^{13,14}$

Moreover, patients with SSD frequently perceive tinnitus in their deaf ear. Tinnitus is the perception of a sound when no external sound is present. The prevalence of tinnitus is reported to be as high as $6-20 \%$ of the general population ${ }^{15}$, with rates increasing among hearing-impaired individuals (up to $67-86 \%$ in bilaterally deaf cochlear implant candidates ${ }^{16}$ ). Although there is less literature on the prevalence of tinnitus in patients with SSD, several studies reported that it is a common symptom in this population. ${ }^{17-21}$ Tinnitus is frequently believed to be the consequence of (central) auditory deprivation due to hearing loss. Cochlear electrical stimulation of the auditory nerve is assumed to have a beneficial influence on tinnitus burden. Indeed, recent studies found lower tinnitus burden following cochlear implantation in bilaterally deaf patients. ${ }^{22}$

Due to these hearing-related difficulties, patients with SSD may experience problems in social interaction and communication, possibly negatively affecting their quality of life $(\mathrm{QoL}){ }^{23-25}$ 


\subsection{Current treatment options for SSD: CROS hearing aids and BCD}

In current clinical practice, there are two treatment options for patients with SSD: Contralateral Routing of Sound (CROS) hearing aids and Bone Conduction Devices (BCDs). Both devices work by the same principle: they transfer the sound from the side of the deaf ear to the functioning contralateral cochlea:

\section{CROS: Contralateral Routing of Sound hearing aid}

A CROS hearing aid consists of two hearing aids which are placed behind the auricle on either side. The hearing aid on the auricle of the deaf ear transmits sounds from the deaf side to the hearing aid on the auricle of the good ear (Figure 3). The hearing aid on the auricle of the good ear then presents these sounds to the tympanic membrane of the good ear via a speaker in the external acoustic meatus.

Figure 3: Schematic depiction of CROS hearing aids.

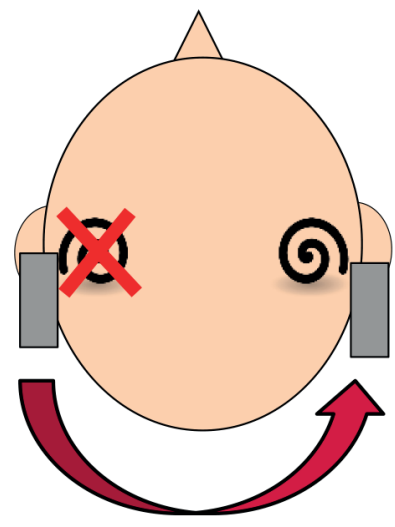

The CROS hearing aid on the auricle of the deaf ear (left side) sends sounds from the deaf side wirelessly to the hearing aid on the auricle of the good ear (right side). 


\section{BCD: Bone Conduction Device}

A BCD transmits the sounds from the deaf side to the good cochlea by vibrating the skull. When the skull vibrates, the perilymph in the functioning cochlea also vibrates, and thus sounds are heard in that cochlea. To let the skull vibrate, a titanium implant is surgically placed in the skull of the patient and the BCD can be mounted (percutaneous skull vibrations; nowadays also transcutaneous BCDs are available). Patients can test the function of a BCD during a trial period, when the BCD is worn on a tight headband (transcutaneous skull vibrations)

Figure 4: Schematic depiction of a surgically implanted BCD.

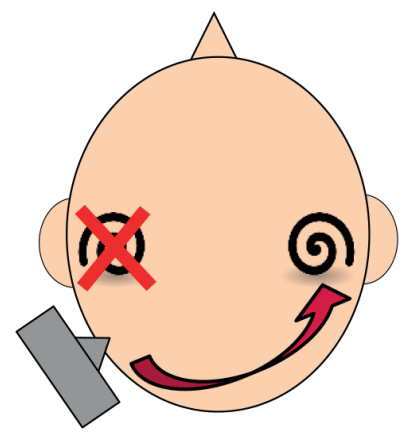

The BCD transmits sounds from the deaf side (left) via vibrations of the skull to the functioning cochlea (on the right side).

\subsection{New treatment option for SSD: cochlear implantation}

In contrast to CROS hearing aids and BCDs, a cochlear implant (CI) provides auditory information to the auditory nerve on the deaf side (Figure 5) and thus restores bilateral input of auditory information to the brain. The electrode array of the cochlear implant directly stimulates the fibers of the auditory nerve in the cochlea electrically. 
Figure 5: Schematic depiction of a CI.

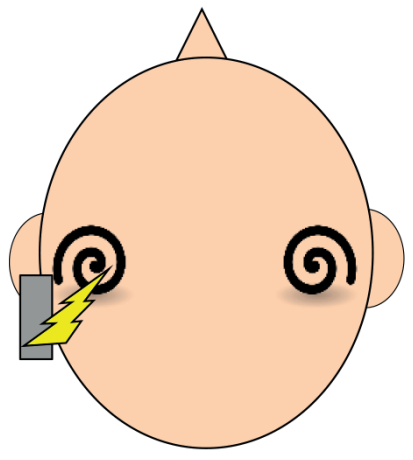

The deaf side (left) now receives direct electrical input and thus bilateral input of auditory information to the brain is restored.

A CI consists of two parts (Figure 6a): an external part, which is visible on the outside of the head, and an internal part, which is implanted during surgery. The external part consists of the microphone, the speech processor and the radio frequent transmitter coil. A radio frequent link conveys sound information and electrical energy from the external part to the internal part. The internal part consists of a subcutaneous radio frequent receiver coil, an electronics package, and a lead wire that runs through the mastoid bone to the cochlea. In the cochlea, an electrode array is placed in the scala tympani (Figure $\mathbf{6 b}$ ). Since the hair cells in the organ of Corti are damaged, the target for stimulation are the spiral ganglion cells (dendrites of the auditory nerve) ${ }^{26}$ 
Figure 6: Schematic overview of a cochlear implant. ${ }^{26}$
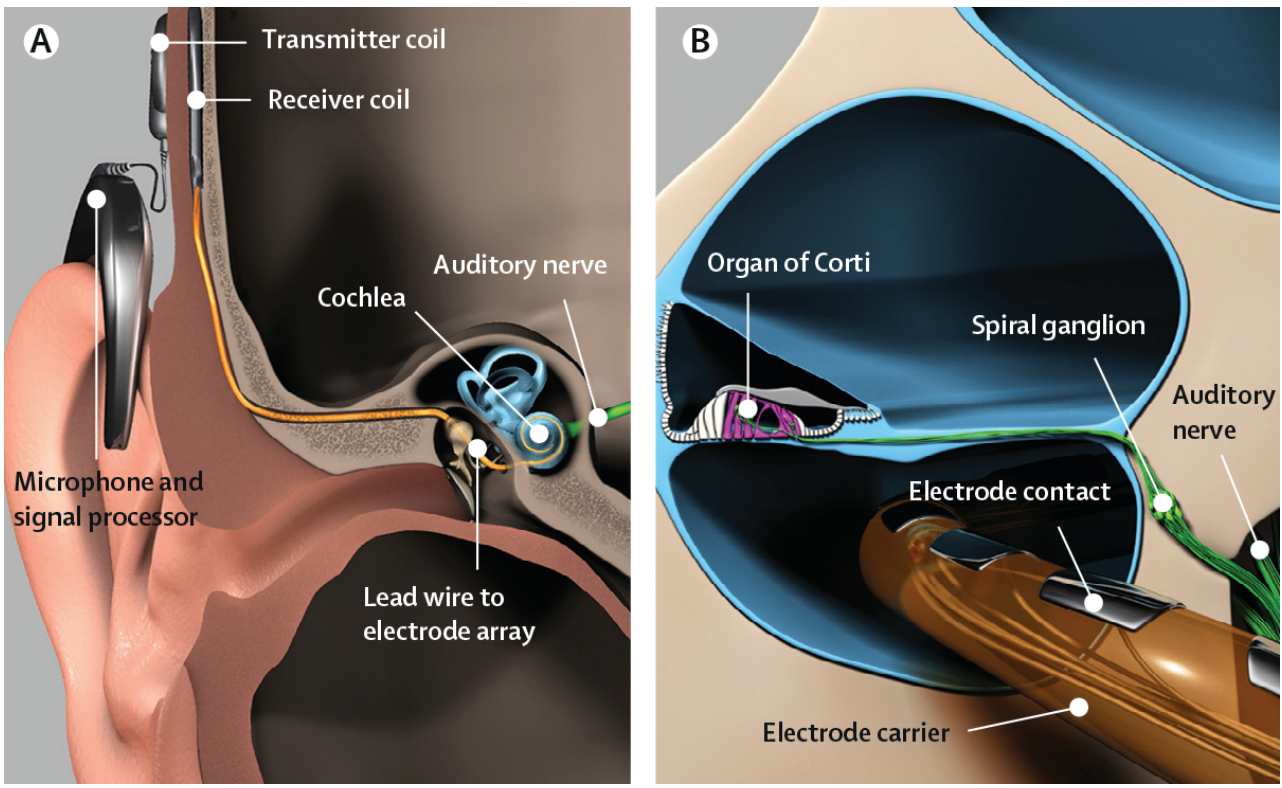

a) A CI consists of an external part, which is visible on the outside of the head. The internal part is implanted during surgery.

b) The electrode array is placed in the scala tympani. Since the hair cells in the organ of Corti are damaged, the target for stimulation are the spiral ganglion cells (dendrites of the auditory nerve).

\subsection{Motivation of research}

Cochlear implantation is considered to be a new treatment option for patients with SSD. With cochlear implantation, bilateral input of auditory information to the brain can be restored, so that the brain is able to make use of the binaural hearing effects. An example of the superiority of hearing with input from two ears (albeit electrical input) was found in a trial evaluating sequential versus simultaneous bilateral cochlear implantation for patients with bilateral sensorineural hearing loss: patients with two CIs had better speech perception in noise with spatially separated sources and were better at localizing sounds than patients who received one CI. ${ }^{27,28}$ 
Theoretically, also for patients with SSD cochlear implantation restores bilateral input and may thus lead to improved speech perception in noise and sound localization. Moreover, electrical stimulation in the deaf ear may suppress tinnitus and therefore decrease tinnitus burden. Following all these benefits, QoL might improve. One must keep in mind, however, that the quality of the sound of a CI is not similar to the quality of acoustic sounds: the brain must be able to fuse two different signals. Furthermore, cochlear implantation is more expensive than treatment with CROS hearing aids or BCDs. Next to that, cochlear implantation is an invasive treatment option, for which patients may not want to opt. All these factors should be taken into account when investigating cochlear implantation for SSD. Ideally, the new treatment option (CI) should be compared to the current clinical practice for patients with SSD, which consists of treatment with CROS hearing aids or BCDs.

\subsection{Comparing therapeutic interventions}

In Evidence-Based Medicine, a Randomised Controlled Trial (RCT) represents the most valuable study design (high "Level of Evidence") to evaluate the effectiveness of therapeutic interventions, since the risk of specific forms of "bias" is minimized. Bias is a systematic error, or deviation from the truth, in results or inferences. ${ }^{29}$ Study results may be influenced by various forms of bias, which can be categorized in:

- Selection bias: selection bias may occur when there is a systematic difference between either the patients that participate in the study and the patients that do not participate in the study (affecting generalizability). Another form of selection bias occurs when systematic differences between baseline characteristics make that groups are not comparable..$^{29}$ Randomization is a good way to prevent this form of bias, provided that the allocation sequence is concealed.

- Information bias: systematic differences in how the outcomes are measured in the study groups may result in information bias. Ideally, both researchers and patients do not know which of the treatment options is applied ("double blind"), so that 
that knowledge can be of no influence on the study results. Unfortunately, blinding is not always possible in research with surgical interventions due to practical and ethical reasons.$^{30}$ Another form of information bias is recall bias: patients cannot reliably answer questions about a situation in the past, which is often the case when a retrospective study is performed. Therefore, data are ideally collected prospectively: a pre-published study protocol states which data will be collected and when. Moreover, a pre-published study protocol may help to reduce publication bias: publication bias is the selective reporting of specific findings and omitting other (insignificant) findings. When a study protocol is published, colleagues can check if all investigated outcomes were reported.

- Confounding: a confounder is a (known or unknown) variable that influences both the dependent and independent variable. An unbiased comparison of treatment options is possible if variables (i.e. possible confounders) are distributed evenly across groups by randomization.

When one wants to compare the treatment options for patients with SSD, it is important to consider these forms of bias to minimize the risk of bias on study results. 


\subsection{Outline of thesis}

\subsubsection{Part I: Introduction and literature reviews}

We first aimed to study the efficacy of the current treatment options for single-sided deafness. In Chapter 2, we systematically reviewed the literature that investigated the effect of CROS hearing aids and BCDs for patients with SSD on the outcomes speech perception in noise, sound localization and QoL. In Chapter 3, we systematically reviewed the literature that reported on cochlear implantation for patients with SSD on the speech perception in noise, sound localization, tinnitus and QoL.

These two chapters formed the background for an RCT to investigate these three treatment options for patients with SSD.

\subsubsection{Part II: CINGLE-trial}

In Chapter 4, we described the research protocol that we used to evaluate the three treatment options for patients with SSD. The results after 3 and 6 months of follow-up for all patients on a subset of outcome measures were presented in Chapter 5.

\subsubsection{Part III: vocoder and pitch match experiments}

SSD patients with a CI and contralateral normal hearing have the unique ability to compare sounds and stimuli between their electric (CI) ear and acoustic ear. In Chapter 6, we aimed to answer the question 'What does a CI sound like?'. We presented speech and music stimuli to the CI ear of SSD patients that were implanted with a CI, and subsequently asked these patients to compare these stimuli to modified versions of these stimuli presented to their normal ear and asked them to rate the similarity between these two stimuli.

We performed other experiments to compare the acoustic and electric in Chapter 7. We activated individual electrode contacts of the electrode array and asked patients to compare the perceived pitch of the individually activated electrode contacts to the pitch of two acoustically presented tones to their normal ear. Subsequently, we asked them to 
select the tone with the pitch closest to the pitch of the activated electrode. Ultimately, we could construct a frequency-place map for the implanted cochlea and learn more about the frequency allocation of cochlear implant electrode arrays. In Chapter 8, a similar analysis was done, but now the effect of time and the type of electrode array could was also assessed.

\subsubsection{Part IV: Quality of reporting}

In Part One of this thesis, two systematic reviews were presented. In Part Two, we described the study protocol and the short-term results of a RCT. These types of studies (systematic reviews and RCTs) may provide high level of evidence when adequately reported so readers can access all relevant and necessary information. As a final part in this thesis, we described two studies in which we evaluated the quality of reporting of systematic reviews (Chapter 9) and RCTs (Chapter 10) in the field of otorhinolaryngology.

\subsubsection{Part V: Discussion}

In Chapter 11, we summarized and discussed the findings of the previous chapters and we provided directions for future research. Chapter 12 is a summary in Dutch of all previous chapters. 


\section{REFERENCES}

\section{Main text}

1. Akeroyd MA. The psychoacoustics of binaural hearing. Int J Audiol 2006;45:S25-S33.

2. Avan P, Giraudet F, Büki B. Importance of binaural hearing. Audiol Neurotol 2015;20(Suppl 1):3-6.

3. Zeitler DM, Dorman MF. Cochlear Implantation for Single-Sided Deafness: A New Treatment Paradigm. J Neurol Surg B Skull Base 2019;80(2):178-186.

4. Middlebrooks JC, Green DM. Sound localization by human listeners. Ann Rev Psychol 1991;42:135-159.

5. Carhart R. Monaural and binaural discrimination against competing sentences. Intern Audiol 1965;4:5-10.

6. MacKeith NW, Coles RRA. Binaural advantages in hearing of speech. J Laryngol Otol 1971;85:213232.

7. Bronkhorst AW, Plomp R. The effect of head-induced interaural time and level differences on speech intelligibility in noise. J Acoust Soc Am 1988;83:1508-1516.

8. Dunn CC, Tyler RS, Oakley S, Gantz BJ, Noble W. Comparison of speech recognition and localization performance in bilateral and unilateral cochlear implant users matched on duration of deafness and age at implantation. Ear Hear 2008;29:325-359.

9. Kamal SM, Robinson AD, Diaz RC. Cochlear implantation in single-sided deafness for enhancement of sound localization and speech perception. Curr Opin Otolaryngol Head Neck Surg 2012;20:393-397.

10. Van Wanrooij MM, Van Opstal AJ. Contribution of Head Shadow and Auricle Cues to Chronic Monaural Sound Localization. J Neuroscience 2004;24:4163-4171.

11. Shaw EAG. Transformation of sound pressure level from the free field to the eardrum in the horizontal plane. J Acoustic Soc Am 1974;56:1848-1861.

12. Festen JM and Plomp R. Speech-reception threshold in noise with one and two hearing aids. J Acoustic Soc Am 1986;79:465-471.

13. Vlastarakos PV, Nazos K, Tavoulari EF, Nikolopoulos TP. Cochlear implantation for singlesided deafness: the outcomes. An evidence based approach. Eur Arch Otorhinolaryngol 2014;271(8):2119-2126.

14. Kitterick PT, Smith SN, Lucas L. Hearing Instruments for Unilateral Severe-to-Profound Sensorineural Hearing Loss in Adults: A Systematic Review and Meta-Analysis. Ear Hear 
2016;37(5):495-507.

15. Bauer CA. Mechanisms of tinnitus generation. Curr Opin Otolaryngol Head Neck Surg 2004;12:413-417.

16. Quaranta N, WagstaffS, Baguley DM. Tinnitus and cochlear implantation. Int J Audiol 2004;43:245251.

17. Van de Heyning P, Vermeire K, Diebl M, Nopp P, Anderson I, De Ridder D. Incapacitating unilateral tinnitus in single-sided deafness treated by cochlear implantation. Annals of Otol, Rhinol and Laryngol 2008;117(9):645-652.

18. Arndt S, Aschendorff A, Laszig R, Beck R, Schild C, Kroeger S, Ihorst G, Wesarg T. Comparison of pseudobinaural hearing to real binaural hearing rehabilitation after cochlear implantation in patients with unilateral deafness and tinnitus. Otol Neurotol 2011;32(1):39-47.

19. Ramos Á, Polo R, Masgoret E, Artiles O, Lisner I, Zaballos ML, Moreno C, Osorio Á. Cochlear implant in patients with sudden unilateral sensorineural hearing loss and associated tinnitus. Acta Otorrinolaringol Esp 2012;63(1):15-20.

20. Galvin JJ 3rd, Fu QJ, Wilkinson EP, Mills D, Hagan SC, Lupo JE, Padilla M, Shannon RV. Benefits of Cochlear Implantation for Single-Sided Deafness: Data From the House Clinic-University of Southern California-University of California, Los Angeles Clinical Trial. Ear Hear 2019;40(4):766781.

21. Peter N, Liyanage N, Pfiffner F, Huber A, Kleinjung T. The Influence of Cochlear Implantation on Tinnitus in Patients with Single-Sided Deafness: A Systematic Review. Otolaryngol Head Neck Surg 2019;161(4):576-588.

22. Ramakers GGJ, van Zon A, Stegeman I, Grolman W. The effect of cochlear implantation on tinnitus in patients with bilateral hearing loss: A systematic review. Laryngoscope 2015;125(11):2584-2592.

23. Wie OB, Pripp AH, Tvete O. Unilateral deafness in adults: effects on communication and social interaction. Annals of Otol Rhinol Laryngol 2010;119(11):772-781.

24. Olze H, Szczepek AJ, Haupt H, Förster U, Zirke N, Gräbel S, Mazurek B. Cochlear implantation has a positive influence on quality of life, tinnitus, and psychological comorbidity. Laryngoscope 2011;121(10):2220-2227.

25. Lucas L, Katiri R, Kitterick PT. The psychological and social consequences of single-sided deafness in adulthood. Int J Audiol 2018;57(1):21-30.

26. Kral A, Kronenberger WG, Pisoni DB, O’Donoghue GM. Neurocognitive factors in sensory restoration of early deafness: a connectome model. Lancet Neurol 2016;15(6):610-621. 
27. Smulders YE, van Zon A, Stegeman I, Rinia AB, Van Zanten GA, Stokroos RJ, Hendrice N, Free RH, Maat B, Frijns JH, Briaire JJ, Mylanus EA, Huinck WJ, Smit AL, Topsakal V, Tange RA, Grolman W. Comparison of Bilateral and Unilateral Cochlear Implantation in Adults: A Randomized Clinical Trial. JAMA Otolaryngol Head Neck Surg 2016;142(3):249-256.

28. Van Zon A, Smulders YE, Stegeman I, Ramakers GGJ, Kraaijenga VJC, Koenraads SP, Van Zanten GA, Rinia AB, Stokroos RJ, Free RH, Frijns JH, Huinck WJ, Mylanus EA, Tange RA, Smit AL, Thomeer HG, Topsakal V, Grolman W. Stable benefits of bilateral over unilateral cochlear implantation after two years: A randomized controlled trial. Laryngoscope 2017;127(5):1161-1168.

29. The Cochrane Collaboration, Cochrane Handbook. Available via https://training.cochrane.org/ handbook/current, accessed June 2020.

30. Boutron I, Tubach F, Giraudeau B, Ravaud P. Blinding was judged more difficult to achieve and maintain in nonpharmacologic than pharmacologic trials. J Clin Epidemiol 2004;57(6):543-550.

\section{Figures}

Figure 1: Maartje Kunen, www.medicalvisuals.nl.

Figure 2: Adapted from: Pearson Education, Benjamin Cummings https://classconnection. s3.amazonaws.com/184/flashcards/1904184/jpg/71352567414440.jpg

Figure 3 to 5: self-made.

Figure 6: Kral A, Kronenberger WG, Pisoni DB, O’Donoghue GM. Neurocognitive factors in sensory restoration of early deafness: a connectome model. Lancet Neurol 2016;15(6):610-621. 



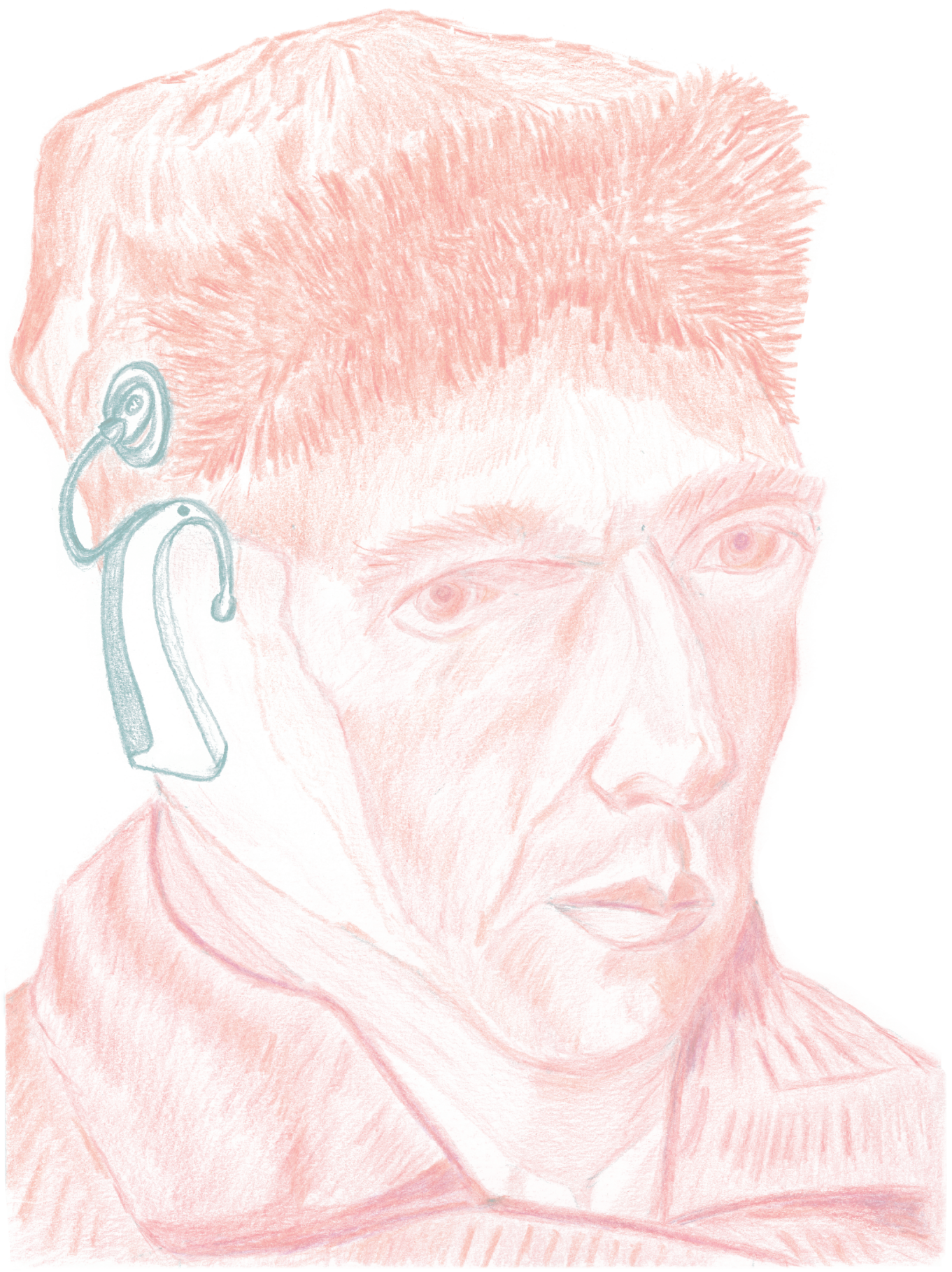




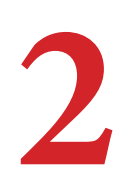

\section{Review: Bone Conduction Devices and \\ Contralateral Routing of Sound Systems in Single-Sided Deafness}




\section{ABSTRACT}

\section{Objective}

Systematically review the literature on the clinical outcome of Bone Conduction Devices (BCD) and Contralateral Routing of Sound Systems (CROSS) for patients with single-sided deafness (SSD).

\section{Data Sources}

PubMed, Embase, Cochrane Library and CINAHL databases were searched up to April $7^{\text {th }}$, 2014.

\section{Review Methods}

All studies investigating BCD and CROSS for patients with SSD on speech perception in noise, sound localization or quality of life were selected and critically appraised.

\section{Results}

In total, 46 articles were retrieved of which six satisfied the eligibility criteria. Critical appraisal showed that five studies $(n=91)$ carried a moderate to high directness of evidence and a low to moderate risk of bias. Subsequently study characteristics and outcome measurements were extracted. Due to large heterogeneity between studies, pooling of data was not feasible. Studies did not show a clear advantage of BCD or CROSS on speech perception in noise. BCD and CROSS lead to the same sound localization ability as the unaided condition. Quality of life did not differ significantly between conditions; however, subjective speech communication did improve. 


\section{Conclusion}

There are no high level of evidence studies comparing BCD and CROSS in patients with SSD. Literature showed no beneficial effect of BCD nor CROSS regarding speech perception in noise and sound localization. Subjective speech communication demonstrated a moderate improvement with BCD and CROSS. High evidence studies comparing all treatment options for single-sided deafness should be conducted. 


\section{INTRODUCTION}

Speech perception in noise and sound localization are well known difficulties for listeners with single-sided deafness (SSD). ${ }^{1,2}$ With one hearing ear and one non-functional ear, patients cannot benefit from binaural squelch (ability of the brain to separate sound and noise signals from spatially separated sources) ${ }^{1,2}$ and summation effects (redundancy of auditory input $)^{3}$. Furthermore, the head shadow effect comes into play: the head acts as an acoustic barrier for the better ear attenuating signals coming from the hearing field of the poor side. ${ }^{4}$ Patients suffering SSD experience problems in their daily life in social interaction and communication. ${ }^{5}$

Nowadays, there are various options for patients suffering SSD such as the Contralateral Routing of Sound System (CROSS) and the Bone Conduction Device (BCD). A CROSS conducts signals from the hearing field of the poor ear via a wire (or FM/Bluetooth) to a microphone in the ear canal of the better ear. Although the ear canal of the better ear is now partly obstructed, sound awareness is restored. A BCD transfers signals from the hearing field of the poor side to the better hearing ear by vibration of the bone of the skull. A trial with a BCD can be performed by attaching the BCD to a tight headband. After this trial period, a titanium screw can be surgically implanted in the skull bone to conduct the signals. Theoretically, CROSS and BCD alleviate the head shadow effect and thus improve speech perception in noise and sound localization and they may also have a beneficial influence on quality of life. However, both modalities do not restore binaural hearing. It is important to know to what extent these modalities help this handicapped population.

Therefore, the aim of our study is to systematically review evidence in the literature on both treatment modalities for patients with single-sided deafness on speech perception in noise, sound localization and quality of life. 


\section{METHODS}

\section{Search strategy}

A systematic search in the PubMed, Embase, Cochrane Library and Cumulative Index to Nursing and Allied Health Literature (CINAHL) databases was conducted from their inception up to April $7^{\text {th }}, 2014$. The search terms were single-sided deafness, bone conduction device and contralateral routing of sound system and their synonyms (see Appendix $\mathbf{1}$ for complete syntax).

There is not an international consensus on the definition of SSD. In this article, we use the following criteria: in patients with SSD, there is a sensorineural hearing loss (HL) with a threshold of $\leq 30 \mathrm{~dB}$ hearing loss (pure tone average [PTA] on $0.5,1,2,4 \mathrm{kHz}$ ) in the better ear (i.e. the better ear does not need a hearing aid) and the poor ear has a sensorineural hearing loss with a threshold of $\geq 70 \mathrm{~dB}$ HL (severe to profound hearing loss). In contrast, asymmetrical hearing loss (AHL) exists when the better ear has a threshold of $\leq 50 \mathrm{~dB} H \mathrm{HL}$ and the poor ear has a threshold of $\geq 70 \mathrm{~dB}$ HL. We think it is important to highlight these differences, since the hearing level of the better ear may influence hearing performance. Nevertheless, in this review we included articles investigating patients with SSD as well as AHL.

To minimize reporting and retrieval bias, no terms related to our outcomes of interest were included in the search. In addition, a related article search was performed.

\section{Study selection}

Authors JPMP and ALS screened titles and abstracts of the retrieved records and subsequently the full-text versions of the potentially relevant articles which evaluated the effect of both BCD and CROSS in patients with SSD. The outcomes of interest were speech perception in noise, sound localization and quality of life. Criteria for inclusion and exclusion are shown in Figure 1. We assessed the eligibility of the trials independently and settled any differences in opinion by discussion. 


\section{Critical appraisal of included studies}

Predefined criteria were used for assessment of the directness of evidence and risk of bias (see

Table 1). Discrepancies were discussed until consensus was reached.

Studies were classified as having high directness of evidence if they complied with five or more criteria, as moderate directness of evidence if they complied with three or four criteria and as low directness of evidence if they complied with two or less criteria. In the assessment of risk of bias, studies were classified as having a low risk of bias if they complied with five or four of the criteria, as a moderate risk of bias with three or two criteria and as a high risk of bias with one or zero criteria. All studies with either a low directness of evidence or a high risk of bias were excluded for further review.

\section{Data extraction and statistics}

Study characteristics and outcome categories of the included studies were extracted and presented in Table 2. To compare speech perception in noise with BCD and CROSS to the unaided condition, p-values were calculated using the Wilcoxon signed-rank test for paired observations when sufficient data was provided.

\section{RESULTS}

\section{Search strategy and study selection}

The search in the PubMed, Embase, Cochrane Library and CINAHL databases retrieved a total of 29 articles. Figure 1 shows how the search results were further assessed. After removal of duplicates and selecting articles by title or abstract, 10 articles were left to read in full-text. 
Figure 1. Flowchart of search (search date April 7, 2014).

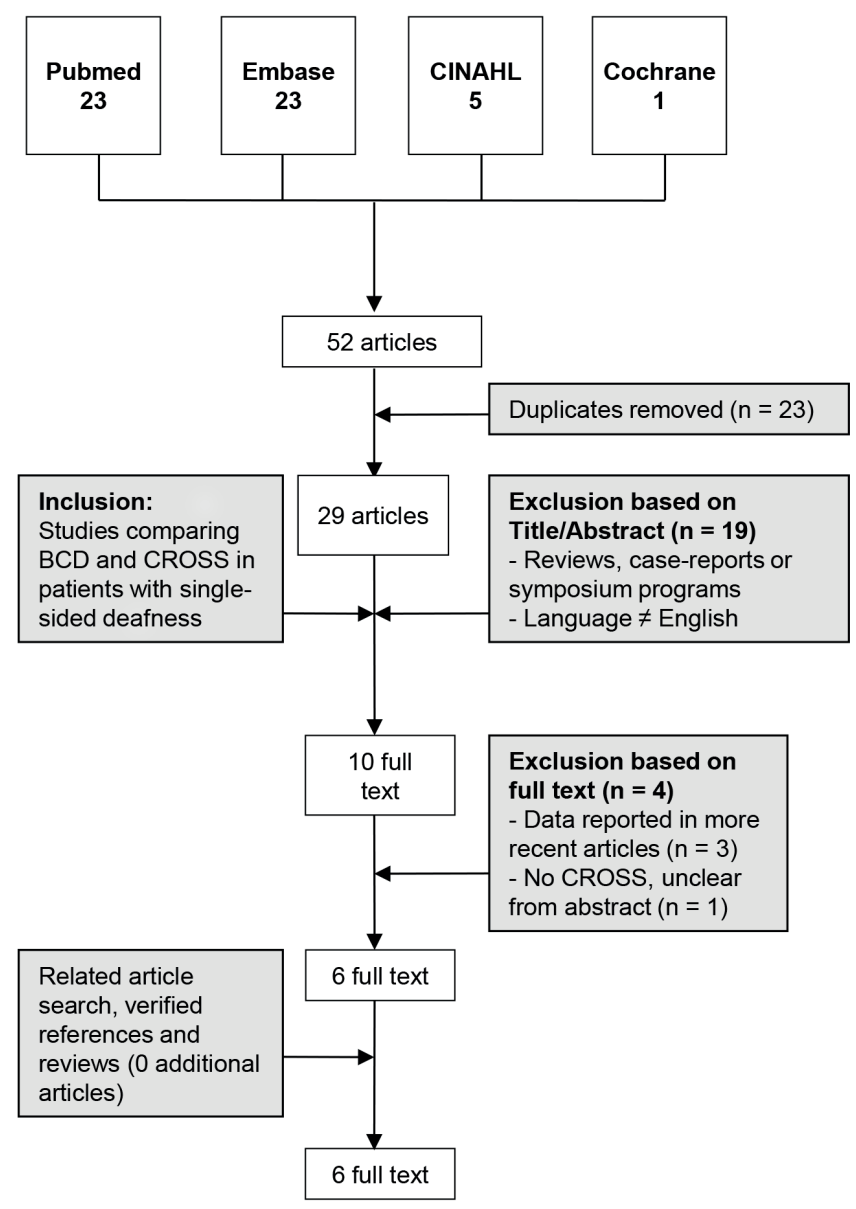

Legend: Abbreviations: BCD: bone conduction device; CROSS: contralateral routing of sound system.

Following full-text assessment, four studies could be discarded. Three studies $^{6-8}$ reported patient data that was also reported in more recent articles by the same group of investigators, which were all included. ${ }^{9,10}$ The fourth study was excluded because there was no data provided on clinical outcome in the CROSS condition, which was unclear from the abstract. ${ }^{11}$ Crossreference checking and related article search yielded no additional articles. Six studies, including a total of 94 patients, were eligible for critical appraisal. 


\section{Critical appraisal of included studies}

Table 1 summarizes the critical appraisal of the six included studies. There were no studies with a low directness of evidence. One study scored a high risk of bias and was therefore excluded. ${ }^{12}$ The other five studies ( $\mathrm{n}=91$ patients) were left for further data extraction and analysis., ${ }^{9,10,13-15}$

\section{Data extraction}

Study characteristics are presented in Table 2 . There is a large degree of clinical heterogeneity amongst and within studies in terms of classification of hearing loss (SSD versus AHL), duration of deafness and follow up duration. Two studies evaluated BCD on a headband, whereas the other studies implanted the BCD. Owing to this heterogeneity, pooling of data was not possible. We therefore summarized the extracted data of the different outcomes (Tables 3 - 6). 
Table 1. Critical appraisal of included studies

\begin{tabular}{|c|c|c|c|c|c|c|c|c|c|c|c|c|c|c|}
\hline \multirow[t]{3}{*}{ Study } & \multirow[b]{3}{*}{ 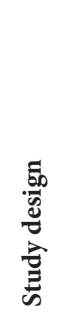 } & \multicolumn{6}{|c|}{ Directness of Evidence } & \multicolumn{6}{|c|}{ Risk of Bias } & \multirow[b]{3}{*}{ 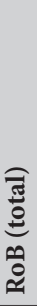 } \\
\hline & & \multirow[b]{2}{*}{ 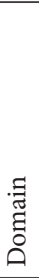 } & \multirow[b]{2}{*}{ 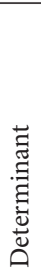 } & \multicolumn{3}{|c|}{ Outcome } & \multirow[b]{2}{*}{$\begin{array}{l}0 \\
\exists \\
3 \\
0 \\
\overline{0} \\
0\end{array}$} & \multirow[b]{2}{*}{ 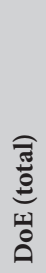 } & \multirow[b]{2}{*}{ 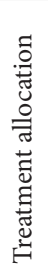 } & \multirow[b]{2}{*}{ : } & \multirow[b]{2}{*}{ 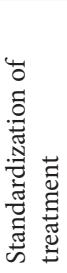 } & \multirow[b]{2}{*}{ 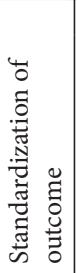 } & & \\
\hline & & & & 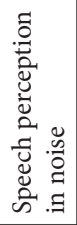 & 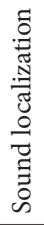 & 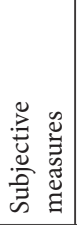 & & & & & & & 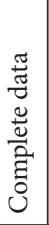 & \\
\hline Arndt $2011^{13}$ & RCS & 0 & D & 0 & 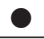 & 0 & $\bigcirc$ & M & $\mathrm{O}$ & 0 & 0 & 0 & 0 & M \\
\hline Hassepass $2013^{12}$ & RCS & 0 & D & ○ & 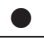 & $\bigcirc$ & $\bigcirc$ & M & O & $\bigcirc$ & $\bigcirc$ & $\bigcirc$ & $\bigcirc$ & $\mathrm{H}$ \\
\hline Hol $2005^{9}$ & PCS & D & 0 & 0 & 0 & 0 & 0 & $\mathrm{H}$ & 0 & 0 & 0 & 0 & 0 & $\mathrm{M}$ \\
\hline Hol $2010^{14}$ & RCT & 0 & D & ? & 0 & - & D & $\mathrm{H}$ & 0 & $\mathrm{O}$ & O & O & 0 & $\mathrm{~L}$ \\
\hline $\operatorname{Lin} 2006^{10}$ & PCS & D & 0 & 0 & 0 & 0 & D & $\mathrm{H}$ & 0 & 0 & 0 & 0 & 0 & $\mathrm{M}$ \\
\hline Wazen $2003^{15}$ & PCS & ○ & 0 & 0 & 0 & 0 & 1 & $M$ & 0 & 0 & 0 & 0 & 0 & $\mathrm{M}$ \\
\hline
\end{tabular}

\section{Legend:}

RCS $=$ Retrospective Case Series

PCS $=$ Prospective Case Series

RCT $=$ Randomized Controlled Trial

$\mathrm{L}=$ low, $\mathrm{M}=$ moderate, $\mathrm{H}=$ high

\section{Directness of evidence (DoE)}

Domain

- All patients have SSD, defined as a sensorineural hearing loss (HL) with a threshold of $\geq 70 \mathrm{~dB} H L$ in the poor ear and a threshold of $\leq 30 \mathrm{~dB} \mathrm{HL}$ in the better ear at frequencies $0.5,1,2,4 \mathrm{kHz}$ (pure tone average).

- Not all patients have sensorineural hearing loss (HL) with a threshold of $\geq 70 \mathrm{~dB}$ HL in the poor ear and a threshold of $\leq 30 \mathrm{~dB}$ HL in the better ear at frequencies $0.5,1,2,4 \mathrm{kHz}$ (pure tone average), but the mean values are within these limits.

$\bigcirc$ Other

\section{Determinant}

- Measurements in the 1) CROSS, 2) implanted percutaneous BCD and 3) unaided situations

- Measurements in the 1) CROSS, 2) BCD on a headband and 3) unaided situations

$\bigcirc$ No measurements in the unaided situation

Outcome

Study reports on:
1. Speech perception in noise:
Yes
$\mathrm{ONo}$

2. Sound localization:

Yes

$\bigcirc$ No

3. Subjective measures:

Yes

No
Follow up

- $\geq 1$ year

(D) $\geq 4 \mathrm{wk},<1 \mathrm{yr}$

$\bigcirc<4 \mathrm{wk}$, or not clearly mentioned

\section{Risk of bias (RoB)}

Treatment allocation:

- Randomized or concealed allocation of treatment

$\bigcirc$ No randomized or concealed allocation of treatment, or not provided

Blinding of intervention:

- Patients and personnel blinded

$\bigcirc$ Only patients blinded, no blinding or not provided

Standardization of treatment:

1. CROSS and BCD types/manufacturers specified,

2. Patients used treatment modality as prescribed and for the complete follow up time

$\bigcirc$ Not standardized as described above or not provided

Standardization of outcome measure:

- Data acquisition after specified follow up time according to research protocol

$\bigcirc$ No standardized data acquisition or not provided

Completeness of data:

- $<15 \%$ missing data

$\bigcirc \geq 15 \%$ missing data or not reported 


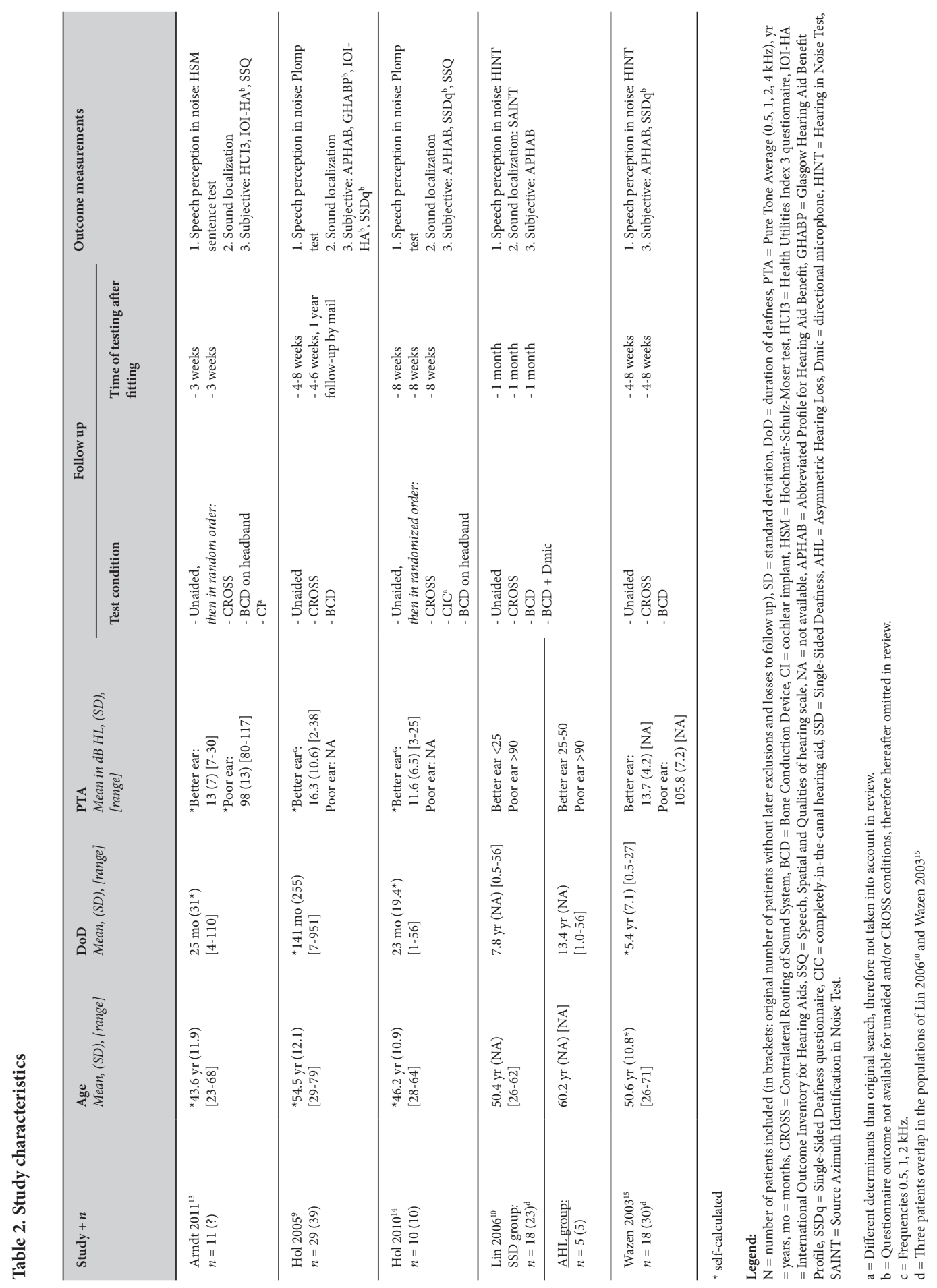


Arndt et al. designed their trial for comparing cochlear implants (CI) to BCD and CROSS, whereas Hol et al. evaluated the completely-in-the-canal (CIC) CROSS hearing aid and Lin et al. investigated the additional value of a directional microphone mounted on the $\mathrm{BCD}$ abutment. ${ }^{10,13,14}$ Since the CI and CIC are different determinants than our original search, we will not take these modalities into account in this review; the directional microphone can be mounted on a BCD and results will be briefly discussed.

\section{Speech perception in noise}

All five studies reported on speech perception in noise $(n=91) .^{9,10,13-15}$ The extracted data are summarized in Table 3.

Table 3. Speech perception in noise

\begin{tabular}{|c|c|c|c|c|c|}
\hline \multirow{2}{*}{ Study } & \multirow{2}{*}{ Configuration } & \multirow{2}{*}{ Unity } & \multicolumn{3}{|c|}{ Condition } \\
\hline & & & Unaided & CROSS & $\mathrm{BCD}$ \\
\hline \multirow{3}{*}{ Arndt $2011^{13}$} & SONO & \multirow{3}{*}{ Word score } & $74.1 \%$ & $76.9 \%$ & $74.1 \%$ \\
\hline & SpeNbe & & $14.6 \%$ & $24.5 \%{ }^{*}$ & $10.4 \%$ \\
\hline & SbeNpe & & $99.5 \%$ & $98.6 \%$ & $98.6 \%$ \\
\hline \multirow{4}{*}{ Hol $2005^{9}$} & S0Nbe & \multirow{4}{*}{ dB SNR } & 2.1 & 1.0 & 0.9 \\
\hline & SONpe & & -2.2 & $0.5^{\star}$ & -1.5 \\
\hline & SbeN0 & & -2.2 & -1.7 & -3.1 \\
\hline & SpeN0 & & 2.2 & $-0.1^{\star}$ & $-0.3^{*}$ \\
\hline \multirow{2}{*}{ Hol $2010^{14}$} & SbeN0 & \multirow{2}{*}{ dB SNR } & -4.3 & -2.7 & -2.0 \\
\hline & SpeN0 & & 0.5 & -1.7 & 0.4 \\
\hline \multirow{3}{*}{$\operatorname{Lin} 2006^{10}$} & SON0 & \multirow{3}{*}{ dB SNR } & \multirow{3}{*}{\multicolumn{3}{|c|}{ No numerical data available }} \\
\hline & S0Nbe & & & & \\
\hline & SoNpe & & & & \\
\hline \multirow{3}{*}{ Wazen $2003^{15}$} & SONO & \multirow{3}{*}{ dB SNR (SD) } & $-1.31 .4)$ & $-1.2(1.6)$ & $-2.6(0.4)$ \\
\hline & S0Nbe & & $0.3(3.2)$ & $-2.1(3.7)$ & $-3.2(2.9)$ \\
\hline & SoNpe & & $-7.13 .2)$ & $-3.4(4.1)$ & $-5.5(4.5)$ \\
\hline
\end{tabular}

\section{Legend:}

* Scores significantly different from other scores in same configuration.

Other values not significant or not reported.

$\mathrm{SNR}=$ signal to noise ratio, $\mathrm{SD}=$ standard deviation

All studies had speakers positioned at $-90,0,90^{\circ}$ azimuth except Arndt et al. ${ }^{13}$ : $-45,0,45^{\circ}$. 
Studies measured speech perception in noise in various configurations of spatially separated speakers: sound $(\mathrm{S})$ or noise $(\mathrm{N})$ comes from the better ear (be), poor ear (pe) or from the front (0 degrees azimuth).

Arndt et al. evaluated CROSS and BCD on a headband using word score percentage on the Hochmair-Schulz-Moser sentence test before patients were implanted with a cochlear implant. ${ }^{13}$ We calculated p-values for the comparison of the unaided, CROSS and BCD conditions. These calculations showed that CROSS performed significantly better than BCD $(\mathrm{p}=0.013)$ and the unaided condition $(\mathrm{p}=0.008)$ in the SpeNbe configuration. In the other configurations, no statistically significant differences were observed.

All other studies measured speech perception in noise with tests resulting in a signal to noise ratio (dB SNR) at which $50 \%$ was understood correctly. In Table 3 , a negative value represents better speech perception in noise ability. Hol et al. found most benefit with the $\mathrm{BCD}$, although only three of the presented scores were significantly different than the other scores in that configuration. ${ }^{9}$ In a later article by that study group, BCD on a headband was investigated. ${ }^{14}$ They found large differences between the various conditions and, depending on the configuration, the best scores for the unaided and CROSS condition. No level of significance was reported. Lin et al. did not report individual or group numerical data, but state that speech perception in noise improved with BCD compared to the unaided and CROSS conditions. ${ }^{10}$ Additionally, they mounted a directional microphone to the BCD abutment in part of their population $(n=13)$ after one month of BCD use. There was no extra benefit of this directional microphone considering speech perception in noise. Finally, Wazen et al. reported best signal to noise ratios in the BCD condition for two configurations. ${ }^{15}$ In the SONpe configuration, the unaided condition performed best, but standard deviations are high.

For each configuration, there is not a general advantage for one of the testing conditions. Moreover, studies do not show one supreme condition for speech perception in noise. 


\section{$\underline{\text { Sound localization }}$}

Four studies evaluated sound localization $(\mathrm{n}=73) .^{9,10,13,14}$ Data of the studies are summarized in Table 4.

Table 4. Sound localization

\begin{tabular}{|c|c|c|c|c|c|c|}
\hline \multirow[t]{2}{*}{ Study } & \multirow[t]{2}{*}{ Test setup } & \multirow[t]{2}{*}{ Configuration } & & \multicolumn{3}{|c|}{ Condition } \\
\hline & & & & Unaided & CROSS & $\mathrm{BCD}$ \\
\hline \multirow[t]{2}{*}{ Arndt $2011^{13}$} & 7 speakers in front from & Correct $^{\mathrm{a}}$ & & $33.9^{\circ}$ & $39.9^{\circ}$ & $30.4^{\circ}$ \\
\hline & $270^{\circ}$ to $90^{\circ}$ with $30^{\circ}$ interval & & & & & \\
\hline \multirow[t]{4}{*}{ Hol $2005^{9}$} & 9 speakers in front from & Correct $^{\mathrm{b}}$ & $500 \mathrm{~Hz}$ & $19.1 \%$ & $16.8 \%$ & $16.5 \%$ \\
\hline & $240^{\circ}$ to $120^{\circ}$ with $30^{\circ}$ & & $3000 \mathrm{~Hz}$ & $18.1 \%$ & $14.6 \%$ & $17.4 \%$ \\
\hline & interval & Lateralization $^{c}$ & $500 \mathrm{~Hz}$ & $45.1 \%$ & $45.1 \%$ & $46.2 \%$ \\
\hline & & & $3000 \mathrm{~Hz}$ & $50.3 \%$ & $42.6 \%$ & $52.6 \%$ \\
\hline \multirow[t]{2}{*}{ Hol $2010^{14}$} & 9 speakers in front from & Lateralization $^{c}$ & $500 \mathrm{~Hz}$ & $53.6 \%$ & $53.0 \%$ & $56.1 \%$ \\
\hline & $\begin{array}{l}240^{\circ} \text { to } 120^{\circ} \text { with } 30^{\circ} \\
\text { interval }\end{array}$ & & $3000 \mathrm{~Hz}$ & $61.0 \%$ & $48.6 \%$ & $58.9 \%$ \\
\hline $\operatorname{Lin} 2006^{10}$ & $\begin{array}{l}5 \text { speakers behind back } \\
\text { from } 135^{\circ} \text { to } 225^{\circ} \text { with } 15 \text { - } \\
25^{\circ} \text { interval }\end{array}$ & Lateralization $^{c}$ & & \multicolumn{3}{|c|}{ No numerical data available } \\
\hline
\end{tabular}

\section{Legend:}

Test setup: arch of speakers positioned at head level in horizontal plane.

Configurations:

Correct - correct speaker identified; Lateralization - located speaker in left or right hemisphere.
a) Chance level $68.6^{\circ}$
b) Chance level $11 \%$
c) Chance level $50 \%$

Arndt et al. measured sound localization error: the difference in degrees between the location of the sound source and the source indicated by the patient. ${ }^{13}$ Sound localization using CROSS and BCD did not differ from unaided localization, but no p-values were reported.

Two articles by Hol et al. asked the patient to first identify the exact correct speaker ('correct') and later only lateralize ('lateralization') the source speaker, using both low and 
high frequency tones. ${ }^{9,14}$ In all three conditions, no scores differed significantly from chance level.

Lin et al. investigated sound localization in only 9 out of 23 patients of their population. ${ }^{10}$ In both quiet and noisy listening conditions, the aided conditions did not lead to a significant benefit in sound localization.

\section{$\underline{\text { Subjective measures }}$}

\section{Abbreviated Profile for Hearing Aid Benefit (APHAB)}

Four of the five studies used the validated APHAB questionnaire to quantify the quality of hearing $(\mathrm{n}=80))^{9,10,14,15}$ Therefore, the results are easily comparable and depicted in a separate table, relative to the unaided condition (Table 5). The APHAB questionnaire consists of three speech communication subscales: ease of communication (EC), listening under reverberant conditions (RV), listening in background noise (BN); and a fourth subscale assessing aversion to sound (AS). All questions contribute to a maximum score of 100 (perfect hearing) or a score of 0 (worst hearing). 
Table 5. Abbreviated Profile for Hearing Aid Benefit (APHAB)

\begin{tabular}{|c|c|c|c|c|}
\hline \multirow{2}{*}{ Study } & \multirow{2}{*}{ Domain } & \multicolumn{3}{|c|}{ Condition } \\
\hline & & Unaided & CROSS & $\mathrm{BCD}$ \\
\hline \multirow{4}{*}{ Hol $2005^{9}$} & $\mathrm{EC}$ & \multirow{4}{*}{$\dagger$} & $-7.2^{*}$ & $-13.1^{\star}$ \\
\hline & $\mathrm{BN}$ & & $-21.1^{*}$ & $-33.1^{*}$ \\
\hline & RV & & $-9.6^{*}$ & $-19.1^{*}$ \\
\hline & AS & & $+12.7^{\star}$ & -3.7 \\
\hline \multirow{4}{*}{ Hol $2010^{14}$} & EC & \multirow{4}{*}{$\dagger$} & -6 & -10 \\
\hline & $\mathrm{BN}$ & & $-8^{\varsigma}$ & $-5^{\S}$ \\
\hline & RV & & -7 & +1 \\
\hline & AS & & +5 & +7 \\
\hline \multirow{4}{*}{$\operatorname{Lin} 2006^{10}$} & EC & \multirow{4}{*}{$\dagger$} & \multirow{4}{*}{\multicolumn{2}{|c|}{ No numerical data available }} \\
\hline & $\mathrm{BN}$ & & & \\
\hline & RV & & & \\
\hline & AS & & & \\
\hline \multirow{4}{*}{ Wazen $2003^{15}$} & $\mathrm{EC}(\mathrm{SD})$ & \multirow{4}{*}{$\dagger$} & $-4.9(15.2)$ & $-6.5(14.2)$ \\
\hline & $\mathrm{BN}(\mathrm{SD})$ & & $-8.9(16.3)$ & $-18.9(17.3)$ \\
\hline & $\mathrm{RV}(\mathrm{SD})$ & & $-9.4(11.8)$ & $-15.6(14.3)$ \\
\hline & AS (SD) & & $+18.1(24.5)$ & $-9.2(28.7)$ \\
\hline
\end{tabular}

\section{Legend:}

$\mathrm{EC}=$ ease of communication, $\mathrm{BN}=$ listening in background noise, $\mathrm{RV}=$ listening in reverberant conditions, $\mathrm{AS}=$ aversiveness of sound

Scores for Hol $2010^{14}$ were calculated by values from bar graph.

$\dagger$ APHAB scores relative to unaided

* Statistically significant, other values not significant or not reported.

${ }^{\S}$ Discrepancy between text and table (table was adopted)

All three studies reporting numerical data found an advantage in both aided conditions on the EC, RV and BN domains.9,14,15 The BCD was more beneficial than CROSS in the studies by Hol et al.9 and Wazen et al.15, whereas Hol et al.14 found more improved scores in the CROSS condition. Lin et al. did not find a beneficial influence of the CROSS, but scores on the $\mathrm{EC}, \mathrm{RV}$ and $\mathrm{BN}$ domains improved with both the $\mathrm{BCD}$ and the $\mathrm{BCD}$ with directional microphone.10 All studies found a deterioration of the performance in the AS domain. Hol et al. also sent the APHAB questionnaire by mail after 1 year of follow up time. In 26 out of 29 patients, no statistically significant differences were found on all domains between the scores after 6 weeks and 1 year of BCD use. ${ }^{9}$ 


\section{Questionnaires exclusive $A P H A B$}

Various other validated questionnaires were used to objectify quality of hearing (Table 6). Arndt et al. evaluated quality of life using the Health Utilities Index 3 (HUI3), showing equivalent outcomes for CROSS and BCD, but no significant difference compared to the unaided condition is mentioned. ${ }^{13}$

Furthermore, in Arndt et al. the quality of life is assessed with the Speech, Spatial and Qualities of hearing scale (SSQ). Hol et al. also evaluated hearing with the SSQ, but only used the Spatial subscale. ${ }^{14}$ Both studies did not find a significant difference between the three conditions.

Table 6. Quality of Life (exclusive APHAB)

\begin{tabular}{|c|c|c|c|c|c|}
\hline \multirow{2}{*}{ Study } & \multicolumn{2}{|c|}{ Questionnaire } & \multicolumn{3}{|c|}{ Condition } \\
\hline & & Domain & Unaided & CROSS & $\mathrm{BCD}$ \\
\hline \multirow{12}{*}{ Arndt $2011^{13}$} & \multirow{9}{*}{ HUI3 } & Vision & 1.00 & 0.99 & 1.00 \\
\hline & & Hearing & 0.80 & 0.80 & 0.89 \\
\hline & & Speech & 1.00 & 1.00 & 1.00 \\
\hline & & Emotion & 0.95 & 0.95 & 0.95 \\
\hline & & Pain & 0.95 & 0.98 & 0.96 \\
\hline & & Ambulation & 1.00 & 1.00 & 1.00 \\
\hline & & Dexterity & 1.00 & 1.00 & 1.00 \\
\hline & & Cognition & 1.00 & 1.00 & 1.00 \\
\hline & & Index & 0.56 & 0.65 & 0.67 \\
\hline & \multirow{3}{*}{ SSQ } & Speech & 2.6 & 3.1 & 2.9 \\
\hline & & Spatial & 2.3 & 2.6 & 2.4 \\
\hline & & Qualities & 5.9 & 5.5 & 5.3 \\
\hline Hol $2010^{14}$ & SSQ & Spatial (SD) & $3.7(1.5)$ & $5.0(1.8)$ & $4.8(2.5)$ \\
\hline
\end{tabular}

Legend:

HUI3 = Health Utilities Index 3 questionnaire, SSQ = Speech, Spatial and Qualities of hearing scale, SD = standard deviation. 


\section{Overall comparison}

In Table 7, the results on all outcomes are summarized to provide a compact overview. For speech perception in noise, there is no clear advantage of one of the conditions. Both treatment modalities and the unaided condition show equal results for sound localization. Quality of life did not differ significantly between conditions, but quality of hearing improved for the speech communication subscales of the APHAB questionnaire.

Table 7. Summary of outcome measures

\begin{tabular}{lllll}
\hline \multirow{2}{*}{ Study } & \multicolumn{4}{l}{ Outcome } \\
\cline { 2 - 5 } & Speech perception in noise & $\begin{array}{l}\text { Sound } \\
\text { localization }\end{array}$ & QoL exclusive APHAB & APHAB \\
\hline Arndt $2011^{13}$ & CROSS $>$ Unaided $>$ BCD & Equal & CROSS $>$ BCD $\sim$ Unaided & - \\
\hline Hol $2005^{9}$ & BCD $>$ Unaided $>$ CROSS & Equal & - & BCD $>$ CROSS $>$ Unaided \\
\hline Hol $2010^{14}$ & Unaided $\sim$ CROSS $>$ BCD & Equal & CROSS $>$ BCD $>$ Unaided & CROSS $>$ BCD $>$ Unaided \\
\hline Lin $2006^{10}$ & BCD $>$ CROSS $\sim$ Unaided & Equal & - & BCD $>$ Unaided $>$ CROSS \\
\hline Wazen $^{15}$ & BCD $>$ Unaided $>$ CROSS & - & - & BCD $>$ CROSS $>$ Unaided \\
\hline
\end{tabular}

Legend

QoL = quality of life, $\mathrm{APHAB}=$ Abbreviated Profile for Hearing Aid Benefit.

Example: in Arndt et al. ${ }^{13}$, speech perception in noise is superior with CROSS compared to the unaided condition and the unaided condition is superior to BCD. On QoL, BCD and the unaided condition score equal, and both less than CROSS.

\section{DISCUSSION}

To our knowledge, this review is the first systematic review conducted on the topic of CROSS and $\mathrm{BCD}$ as a treatment for single-sided deafness. Its strengths are a transparent search strategy, study selection and critical appraisal of selected studies.

The outcomes for speech perception in noise varied across studies, configurations and conditions. Both CROSS and BCD were expected to alleviate the head shadow effect, leading to better speech perception in noise in some conditions. However, we did not observe a uniform improvement across configurations in the aided conditions versus the unaided condition. All studies investigating sound localization found equal performance in both 
aided and unaided conditions. On quality of hearing, all studies indicated an advantage in the aided condition over the unaided condition, but CROSS and BCD were alternatingly preferred. Statistical significance for all outcomes was rarely reported. Quality of life did not differ significantly between conditions.

These results should be interpreted taking the limitations of the included studies into account. First, no randomized controlled trials comparing implanted percutaneous BCD to CROSS and the unaided condition have been conducted. The only study randomizing their population used a cross-over design evaluating BCD on a headband, CROSS and the unaided condition. ${ }^{14}$ All other studies were retrospective or prospective case series, subject to bias. This paucity of high level of evidence studies prevented us from drawing accurate conclusions. In addition, $\mathrm{BCD}$ on headband leads to a difference in bone conduction thresholds up to $15 \mathrm{~dB}$ in the higher frequencies compared to a percutaneously implanted BCD. ${ }^{16,17}$ We found that both studies comparing BCD on headbands to CROSS and the unaided conditions measured worse speech perception in noise than studies evaluating a percutaneously implanted BCD. ${ }^{13,14}$ The performances of these populations may therefore not reflect final performance.

Second, there is a large degree of clinical heterogeneity in the populations of the included studies, therefore pooling of data was not feasible. Both within and between studies, for instance, there was a large range of duration of deafness (4 to 951 months). The grade of hearing loss also varied; individuals with mild hearing loss in the better ear (PTA $\leq 30 \mathrm{~dB} H \mathrm{HL})$ may suffer less from their hearing loss than individuals with moderate hearing loss (PTA $\leq 50$ dB HL), leading to different scores in outcome. Moreover, not all results of the total included population were described without providing reason why they were omitted, which may lead to bias..$^{10,15}$

Finally, studies lack long term audiometric follow up. Maximum audiometric follow up was eight weeks after CROSS or BCD fitting. In the only study with a follow up time of one year, a postal version of the APHAB was sent, showing unchanged scores compared to the follow up moment 6 weeks after fitting. ${ }^{9}$ 
Two other, non-systematic, reviews have been published on this topic. Baguley et al. reviewed four unique studies ${ }^{6-8,15}$ and concluded BCD to be superior to CROSS and CROSS superior to the unaided condition considering speech perception in noise and on the APHAB questionnaire. ${ }^{18}$ No difference in sound localization was observed between the conditions. However, they stressed that their results should be interpreted with consideration of selection bias, small patient numbers, double reporting of patients and unclear inclusion criteria in the included studies. Four years later, Bishop et al. reaffirmed these findings on all three outcomes. ${ }^{19}$ They state that the additional articles used in their review ${ }^{9,10}$, often being an enlargement of the patient populations of the same investigational groups, had not satisfactorily addressed the methodological shortcomings highlighted in the review by Baguley et al. They plead for prospective, randomized studies with longer follow up periods and urge researchers to take preoperative burden into account.

Our results agree with the two previous reviews on sound localization and quality of hearing. However, the extra studies included in our review showed that patients did not benefit from neither BCD nor CROSS regarding speech perception in noise. Despite the recommendations of these reviews, our systematic review noted the same methodological deficiencies in studies in this field as earlier identified, being selection bias, small sample sizes, double reporting of patients and unclear inclusion criteria. Moreover, no recent articles comparing CROSS and BCD in unilaterally deaf patients have been published. This is particularly surprising considering the enormous advances that have been made in hardware and software of both modalities. Furthermore, cochlear implantation could be a new treatment option and for the first time actually restore binaural hearing. ${ }^{13,19}$ To be able to value this new treatment modality, we should first have high quality evidence on the clinical outcome of the current therapy strategies for patients with SSD. 


\section{CONCLUSION}

Our systematic review showed that there are no high level of evidence studies comparing BCD and CROSS in patients with SSD. Based on moderate level of evidence studies, neither BCD nor CROSS provides benefit regarding speech perception in noise and sound localization. Quality of life did not improve, however on speech communication subscales a benefit was assessed. High level of evidence studies comparing all treatment options for single-sided deafness should be conducted.

\section{Appendix 1: search syntax}

Pubmed:

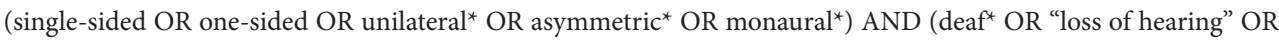
(hearing AND (impair ${ }^{\star}$ OR loss OR disorder $\left.{ }^{\star}\right)$ ))

AND

((bone AND anchored AND hearing AND (implant* OR aid ${ }^{*}$ OR devic ${ }^{*}$ OR system*)) OR (bone AND conducti* AND (implant ${ }^{\star}$ OR aid ${ }^{*}$ OR devic OR system $\left.^{\star}\right)$ ) OR (BAHA) OR (BCD) OR (BAHS) OR (BAHI)) AND ((contralateral AND routing AND (sound ${ }^{*}$ OR signal $\left.\left.{ }^{\star}\right)\right)$ OR (CROS))

Search syntax used in Title and/or Abstract fields.

Search date: April $7^{\text {th }}, 2014$.

Modelled search strategy designed for Embase, CINAHL and Cochrane. 


\section{REFERENCES}

1. Middlebrooks JC, Green DM. Sound localization by human listeners. Ann Rev Psychol 1991;42:135-159.

2. Akeroyd MA. The psychoacoustics of binaural hearing. Int J Audiol 2006;45:S25-S33.

3. Dunn CC, Tyler RS, Oakley S, Gantz BJ, Noble W. Comparison of Speech Recognition and Localization Performance in Bilateral and Unilateral Cochlear Implant Users Matched on Duration of Deafness and Age at Implantation. Ear \& Hearing 2008;29(3):325-359.

4. Van Wanrooij MM, Van Opstal AJ. Contribution of Head Shadow and Pinna Cues to Chronic Monaural Sound Localization. J Neuroscience 2004;24(17):4163-4171.

5. Wie OB, Pripp HP, Tvete O. Unilateral Deafness in Adults: Effects on Communication and Social Interaction. The Annals of Otology, Rhinology, and Laryngology 2010;119(11):772-781.

6. Bosman AJ, Hol MKS, Snik AFM, Mylanus EAM, Cremers CWRJ. Bone-anchored hearing aids in unilateral inner ear deafness. Acta Oto-laryngologica 2003;123(2):258-260.

7. Hol MKS, Bosman AJ, Snik AFM, Mylanus EAM, Cremers CWRJ. Bone-anchored hearing aid in unilateral inner ear deafness: a study of 20 patients. Audiology \& Neurotology 2004;9(5):274-281.

8. Niparko JK, Cox KM, Lustig LR. Comparison of the bone anchored hearing aid implantable hearing device with contralateral routing of offside signal amplification in the rehabilitation of unilateral deafness. Otology \& Neurotology 2003;24(1):73-78.

9. Hol MKS, Bosman AJ, Snik AFM, Mylanus EAM, Cremers CWRJ. Bone-anchored hearing aids in unilateral inner ear deafness: an evaluation of audiometric and patient outcome measurements. Otology \& Neurotology 2005;26(5):999-1006.

10. Lin LM, Bowditch S, Anderson MJ, May B, Cox KM, Niparko JM. Amplification on the Rehabilitation of Unilateral Deafness: Speech in Noise and Directional Hearing Effects with BoneAnchored Hearing and Contralateral Routing of Signal Amplification. Otology \& Neurotology 2006;27:172-182.

11. Hol MKS, Kunst SJW, Snik AFM, Bosman AJ, Mylanus EAM, Cremers CWRJ. Bone-anchored hearing aids in patients with acquired and congenital unilateral inner ear deafness (Baha CROS): clinical evaluation of 56 cases. Annals of Otology, Rhinology, and Laryngology 2010;119(7):447454.

12. Hassepass F, Schild C, Aschendorff A, Laszig R, Maier W, Beck R, Wesarg T, Arndt S. Clinical outcome after cochlear implantation in patients with unilateral hearing loss due to labyrinthitis 
ossificans. Otology \& Neurotology 2013;34(7):1278-1283.

13. Arndt S, Aschendorff A, Laszig R, Beck R, Schild C, Kroeger S, Ihorst G, Wesarg T. Comparison of pseudobinaural hearing to real binaural hearing rehabilitation after cochlear implantation in patients with unilateral deafness and tinnitus. Otology \& Neurotology 2011;32(1):39-47.

14. Hol MKS, Kunst SJW, Snik AFM, Cremers CWRJ. Pilot study on the effectiveness of the conventional CROS, the transcranial CROS and the BAHA transcranial CROS in adults with unilateral inner ear deafness. Eur Arch Otorhinolaryngol 2010;267:889-896.

15. Wazen JJ, Spitzer JB, Ghossaini SN, Fayad JN, Niparko JK, Cox K, Brackmann DE, Soli SD. Transcranial contralateral cochlear stimulation in unilateral deafness. Otolaryngology - Head and Neck Surgery 2003;129(3):248-254.

16. Gruender I, Seidl RO, Ernst A, Todt I. [Relative value of BAHA testing for the postoperative audiological outcome] (German). HNO, 2008;56:1020-1024.

17. Verstraeten N, Zarowski AJ, Somers T, Riff D, Offeciers EF. Comparison of the audiologic results obtained with the bone-anchored hearing aid attached to the headband, the testband, and to the snap abutment. Otology \& Neurotology 2008;30:70-75.

18. Baguley DM, Bird J, Humphriss RL, Prevost AT. The evidence base for the application of contralateral bone anchored hearing aids in acquired unilateral sensorineural hearing loss in adults. Clinical Otolaryngology 2006;31(1):6-14.

19. Bishop CE, Eby TL. The current status of audiologic rehabilitation for profound unilateral sensorineural hearing loss. Laryngoscope 2010;120(3):552-556. 



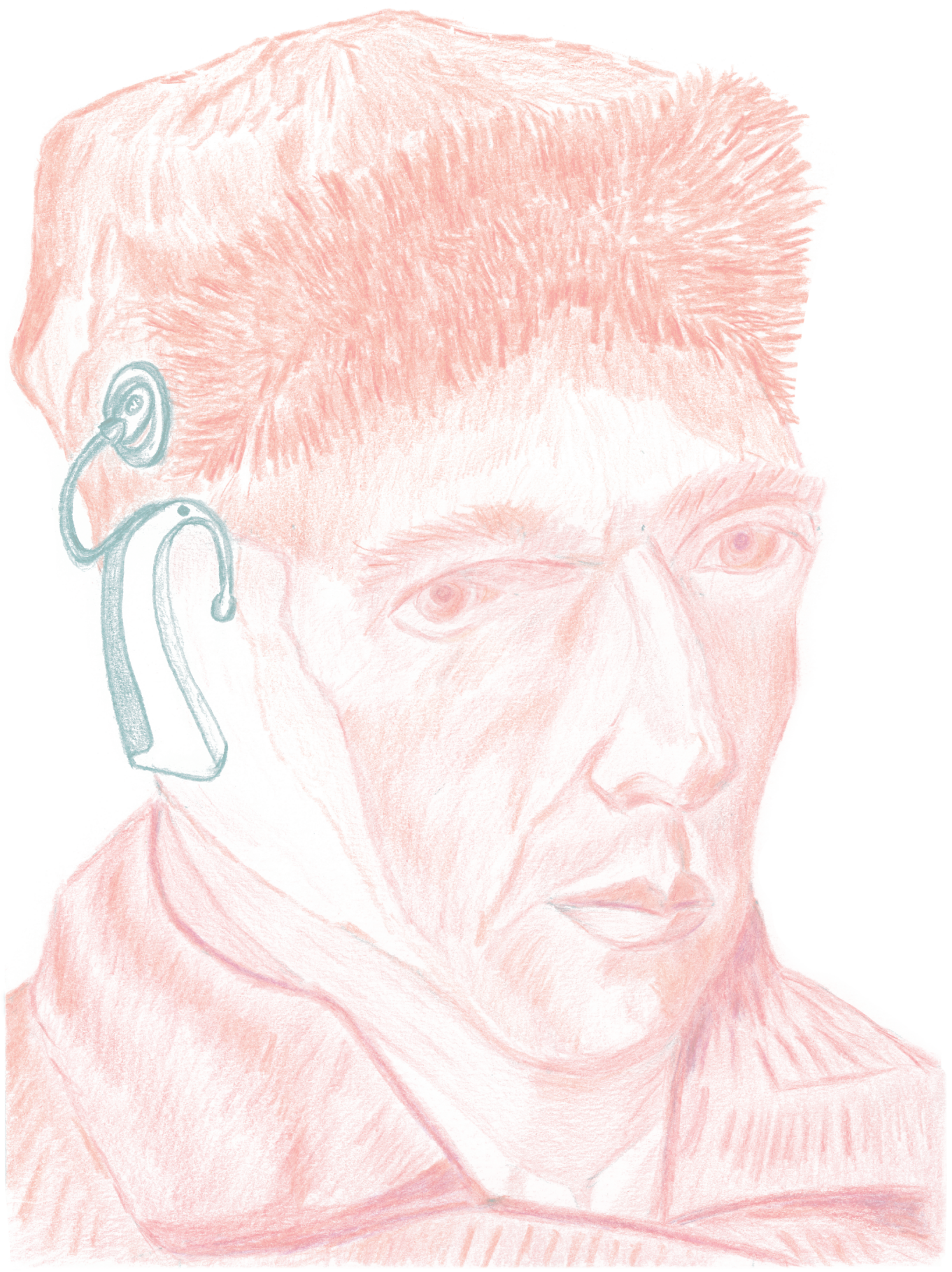




\section{Cochlear Implantation for Patients With Single-Sided Deafness or Asymmetrical Hearing Loss:}

A Systematic Review of the Evidence 


\section{ABSTRACT}

Objective: A systematic review of the literature to evaluate the clinical outcome of cochlear implantation for patients with single-sided deafness (SSD) or asymmetrical hearing loss (AHL).

Data sources: We searched the PubMed, Embase, Cochrane Library and CINAHL databases from their inception up to December $10^{\text {th }}, 2013$ for SSD or AHL and cochlear implantation or their synonyms.

Study selection: In total, 781 articles were retrieved, of which 15 satisfied the eligibility criteria. Our outcomes of interest were: speech perception in noise, sound localization, quality of life (QoL) and tinnitus.

Data extraction: Critical appraisal showed that six studies reported on less than five patients or that they carried a low directness of evidence or a high risk of bias. Therefore we extracted the data of nine studies $(n=112)$. Patient numbers, age, duration of deafness, classification of deafness, pure tone audiometry, follow-up duration and outcome measurements were extracted from all nine articles.

Data synthesis: Due to large heterogeneity between studies, we were not able to pool data in a meta-analysis. We therefore summarized the results of the studies specified per outcome.

Conclusion: There are no high level of evidence studies concerning cochlear implantation in patients with SSD or AHL. Current literature suggests important benefits of cochlear implantation regarding sound localization, QoL and tinnitus. Varying results were reported for speech perception in noise, possibly caused by the large clinical heterogeneity between studies. Larger and high quality studies are certainly warranted. 


\section{BACKGROUND}

Single-sided deafness (SSD) is defined as a condition in which an individual has nonfunctional hearing on one side and normal hearing on the contralateral side. Patients who develop SSD become aware of the importance of binaural hearing in their daily life in terms of social interaction and communication. ${ }^{1}$

Binaural hearing has been proven to be superior to unilateral hearing with regard to speech perception in noise and sound localization..$^{2-5}$ The advantages that normal-hearing listeners gain from binaural hearing are based on three principles: 1) the squelch effect (ability of the brain to separate sound and noise signals from spatially separated sources $)^{4,6}, 2$ ) the binaural summation effect (redundancy of auditory input ${ }^{7}$, and 3) the head shadow effect (better signal-to-noise ratio) ${ }^{8}$.

Current clinical practice for patients with SSD consists of optimizing hearing with either a Contralateral Routing of Signal (CROS) or a Bone Conduction Device (BCD). Both devices are effective in addressing the head shadow effect and thus restoring sound awareness to the deaf side, but they do not provide bilateral auditory input, which is needed for actual binaural hearing.

The limitations of CROS or BCD may be overcome by providing a cochlear implant (CI). During the last decades, cochlear implantation has become a widely accepted intervention for patients with bilateral sensorineural hearing loss (HL) and the selection criteria for implantation have been broadened. Recently, Van Schoonhoven et al. reviewed current literature on bilateral cochlear implantation and concluded that patients with bilateral sensorineural HL perform better on sound localization tests when patients were implanted bilaterally compared to unilaterally. ${ }^{9}$

According to these findings in bilateral deaf patients, the hypothesis is that due to the restored bilateral auditory input, spatial hearing will also improve after cochlear implantation in patients with unilateral deafness, including patients with SSD or asymmetrical hearing loss (AHL). Next to these audiological benefits, cochlear implantation may result in suppression 


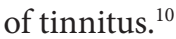

Until now, the clinical outcome of cochlear implantation for patients with SSD or AHL has not been reviewed systematically in detail.

\section{METHODS}

This systematic review conformed to the Preferred Reporting Items for Systematic Reviews and Meta-Analyses (PRISMA) statement, a 27-item checklist to assure clear and transparent reporting of systematic reviews. ${ }^{11,12}$

\section{Search strategy}

A systematic search in the PubMed, Embase, Cochrane Library and Cumulative Index to Nursing and Allied Health Literature (CINAHL) databases was conducted on December $10^{\text {th }}, 2013$. The main search terms were SSD or AHL and cochlear implantation and their synonyms (see Table 1 for complete syntaxes). To minimize reporting and retrieval bias, no terms related to our outcome were included in the search. In addition, a cross-reference and related article search was performed.

Table 1. Search syntax (date of search: December $10^{\text {th }} 2013$ ).

\begin{tabular}{|c|c|c|c|}
\hline Database & Search & Syntax & Results \\
\hline \multirow{4}{*}{ PubMed } & $\# 1$ & $\begin{array}{l}\text { single-sided }\left[\text { tiab] OR one-sided }\left[\text { tiab] OR unilateral }{ }^{*}[\text { tiab] OR }\right.\right. \\
\left.\text { asymmetric* }^{*} \text { tiab] OR monaural }{ }^{*}[\text { tiab }]\right)\end{array}$ & \multirow{4}{*}{352} \\
\hline & $\# 2$ & $\begin{array}{l}\text { deaf }^{*}\left[\text { tiab] OR "loss of hearing" [tiab] OR (hearing[tiab] AND (impair* }{ }^{*} \text { tiab] }\right. \\
\text { OR loss[tiab] OR disorder }{ }^{*}[\text { tiab])) }\end{array}$ & \\
\hline & \#3 & cochlear implant* ${ }^{\star}[$ tiab $]$ & \\
\hline & $\# 4$ & $\# 1 \mathrm{AND} \# 2 \mathrm{AND} \# 3$ & \\
\hline Embase & \multicolumn{2}{|c|}{$\begin{array}{l}\text { Modeled search strategy designed for Embase, in Title/Abstract fields. } \\
\text { (Limited to Embase database only) }\end{array}$} & 338 \\
\hline Cochrane & \multicolumn{2}{|c|}{ Modeled search strategy designed for Cochrane, in Title/Abstract fields. } & 15 \\
\hline CINAHL & \multicolumn{2}{|c|}{ Modeled search strategy designed for CINAHL, in Title/Abstract fields. } & 76 \\
\hline
\end{tabular}




\section{Study selection}

AvZ and JPMP screened titles and abstracts of the retrieved records and subsequently the full- text versions of the potentially relevant articles which evaluated the effect of cochlear implantation in patients with unilateral hearing loss. Criteria for inclusion and exclusion are shown in Figure 1. Although we aimed to focus on the effect of cochlear implantation for patients with SSD, we also included studies in which patients with AHL were investigated. This was done, because there is no international consensus on the definitions for SSD and AHL. In this study, we define SSD as a sensorineural hearing loss with a threshold equal to or greater than $70 \mathrm{~dB}$ HL in the affected ear and a threshold equal to or less than $30 \mathrm{~dB} H \mathrm{HL}$ in the better ear. AHL is defined as a sensorineural hearing loss with a threshold equal to or greater than $70 \mathrm{~dB} H L$ in the affected ear and a threshold less than $70 \mathrm{~dB} H L$ in the better ear.

We assessed the eligibility of the trials independently and settled any differences in opinion by discussion. The outcomes of interest were: speech perception in noise, sound localization, quality of life (QoL) and tinnitus.

\section{Assessing quality of studies}

Predefined criteria were used for assessment of the directness of evidence and risk of bias of the selected studies (see Table 2). Discrepancies were discussed until consensus was reached. All studies with a sample size of less than five patients or with either a low directness of evidence or a high risk of bias were excluded for further review. Studies were classified as having high directness of evidence if they complied with all four criteria, as moderate directness of evidence if they complied with three criteria and as low directness of evidence if they complied with only one or two criteria. If studies complied with all five or four criteria used to assess risk of bias, they were classified as having a low risk of bias, with three as a moderate risk of bias and with one or two as a high risk of bias. 


\section{Data extraction}

Study characteristics and outcome data of the included studies were extracted by the first two authors, disagreements were resolved by discussion. Study characteristics are presented in Table 3.

\section{RESULTS}

\section{Search strategy and study selection}

The search in the PubMed, Embase, Cochrane Library and CINAHL databases retrieved a total of 781 articles. Figure 1 shows how the search results were further assessed. After removal of duplicates and selecting articles by title or abstract, 23 articles were left to read in full-text.

Eight more studies could be discarded following full-text assessment. Three studies presented study data which were also presented in more recent articles included in this review $^{13-15}$, in two studies all patients were CI users, but the effect of cochlear implantation was not evaluated ${ }^{16,17}$, two studies were only published in abstract ${ }^{18,19}$, and one study could not be retrieved in full-text ${ }^{20}$. Cross-reference checking and related article search yielded one additional article, however, this study was excluded because it was only published in abstract form $^{21}$. Fifteen studies, including a total of 166 patients, were eligible for critical appraisal. 
Figure 1. Study selection process

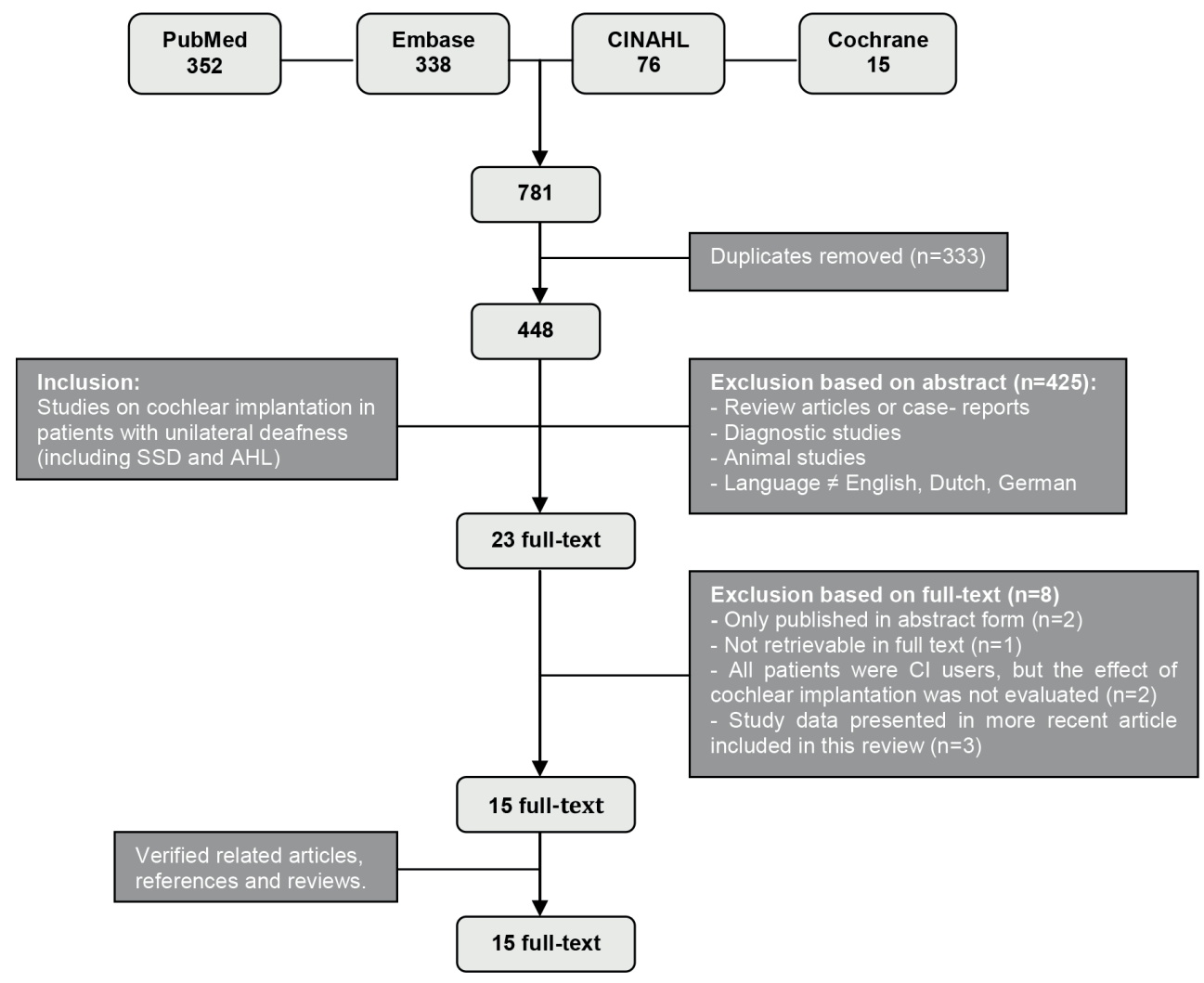

Legend: SSD: single-sided deafness, AHL: asymmetrical hearing loss, CI: cochlear implant.

\section{Assessing quality of studies}

The critical appraisal of the 15 studies is presented in Table 2. All studies evaluated the effect of cochlear implantation on at least one of our outcomes of interest. Three studies reported on less than five patients. ${ }^{22-24}$ We therefore excluded these studies for further review.

Important limitations in the directness of evidence were found in two studies and therefore we did not further review these studies. ${ }^{25,26}$ Both studies scored low on patient population, they assessed only one of our outcomes of interest and there was no follow-up moment at either 6 or 12 months. 
Table 2. Assessment of quality of included studies.

\begin{tabular}{|c|c|c|c|c|c|c|c|c|c|c|c|c|c|c|c|c|}
\hline & \multirow[b]{3}{*}{ 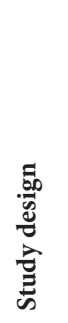 } & \multirow[b]{3}{*}{ 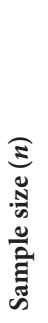 } & \multicolumn{7}{|c|}{ Directness of evidence (DoE) } & \multirow[b]{3}{*}{ 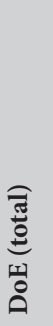 } & \multicolumn{5}{|c|}{ Risk of bias (RoB) } & \multirow[b]{3}{*}{ 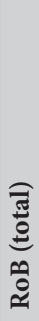 } \\
\hline & & & \multirow[b]{2}{*}{ 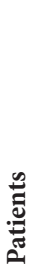 } & \multirow[b]{2}{*}{ 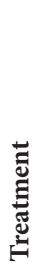 } & \multicolumn{3}{|c|}{ Outcome } & \multicolumn{2}{|c|}{ Follow-up } & & \multirow{2}{*}{ 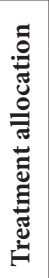 } & \multirow[b]{2}{*}{$\stackrel{00}{:}$} & \multirow[b]{2}{*}{ 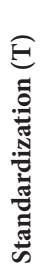 } & \multirow[b]{2}{*}{ 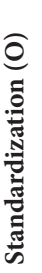 } & \multirow[b]{2}{*}{ 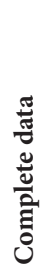 } & \\
\hline & & & & & 苞 & $\stackrel{\mathscr{Z}}{\stackrel{\Xi}{\Xi}}$ & 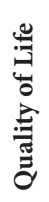 & 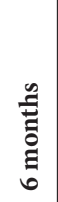 & 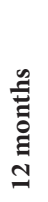 & & & & & & & \\
\hline Arndt 2011 & PCS & 11 & 0 & 0 & 0 & 0 & 0 & 0 & $\bigcirc$ & $\mathbf{H}$ & 0 & 0 & 0 & 0 & 0 & M \\
\hline Buechner 2010 & PCS & 5 & $?$ & 0 & 0 & 0 & $\mathrm{O}$ & 0 & 0 & M & 0 & $\mathrm{O}$ & 0 & 0 & 0 & M \\
\hline Cadieux 2013 & PCS & 5 & $\mathrm{O}$ & O & 0 & 0 & 0 & 0 & $\mathrm{O}$ & $\mathbf{L}$ & $\mathrm{O}$ & $\mathrm{O}$ & 0 & O & $\mathrm{O}$ & $\mathbf{H}$ \\
\hline Firszt 2012 & PCS & 10 & $\mathrm{O}$ & - & 0 & $\mathrm{O}$ & 0 & 0 & $\mathrm{O}$ & M & $\mathrm{O}$ & $\mathrm{O}$ & 0 & - & 0 & $\mathbf{M}$ \\
\hline Hansen 2013 & PCS & 29 & $\mathrm{O}$ & 0 & 0 & O & O & ○ & 0 & M & $\bigcirc$ & O & 0 & 0 & 0 & $\mathbf{H}$ \\
\hline Hassepass 2013 ${ }^{a}$ & PCS & 3 & 0 & - & 0 & 0 & ○ & - & 0 & $\mathrm{H}$ & $\mathrm{O}$ & $\mathrm{O}$ & 0 & 0 & $\mathrm{O}$ & $\mathrm{H}$ \\
\hline Hassepass $2013^{b}$ & PCS & 3 & O & 0 & 0 & 0 & 0 & O & 0 & $\mathbf{H}$ & $\bigcirc$ & $\mathrm{O}$ & 0 & 0 & 0 & $\mathbf{M}$ \\
\hline Jacob 2011 & RCS & 13 & 0 & 0 & 0 & $\mathrm{O}$ & $\mathrm{O}$ & $\mathrm{O}$ & $\mathrm{O}$ & M & $\mathrm{O}$ & $\mathrm{O}$ & 0 & 0 & 0 & $\mathbf{M}$ \\
\hline Kleine Punte 2013 & PCS & 7 & $?$ & 0 & 0 & 0 & 0 & 0 & $\mathrm{O}$ & $\mathbf{M}$ & 0 & 0 & 0 & 0 & 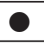 & $\mathbf{M}$ \\
\hline Mertens 2013 & PCS & 15 & 0 & 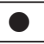 & 0 & ? & 0 & 0 & 0 & M & $\mathrm{O}$ & 0 & 0 & 0 & 0 & M \\
\hline Ramos 2011 & PCS & 10 & $\bigcirc$ & ○ & 0 & ? & 0 & 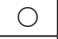 & $\mathrm{O}$ & $\mathbf{L}$ & $\bigcirc$ & $\mathrm{O}$ & 0 & 0 & ○ & M \\
\hline Stelzig 2011 & RCS & 4 & 0 & 0 & 0 & O & 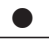 & $\bigcirc$ & $\bigcirc$ & M & 0 & $\mathrm{O}$ & 0 & 0 & 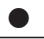 & $\mathbf{M}$ \\
\hline Távora-Vieira 2013 & PCS & 9 & 0 & 0 & 0 & 0 & 0 & 0 & 0 & $\mathbf{M}$ & 0 & 0 & 0 & 0 & 0 & $\mathbf{M}$ \\
\hline Van de Heyning 2008 & PCS & 22 & $\mathrm{O}$ & 0 & $\mathrm{O}$ & ? & $\mathrm{O}$ & 0 & 0 & M & $\mathrm{O}$ & $\mathrm{O}$ & 0 & 0 & 0 & $\mathbf{M}$ \\
\hline Vermeire 2009 & PCS & 20 & $\mathrm{O}$ & 0 & 0 & $\mathrm{O}$ & ○ & O & 0 & $\mathbf{M}$ & O & $\mathrm{O}$ & 0 & ○ & O & M \\
\hline
\end{tabular}

\section{Legend}

Study Design

$\mathrm{RCS}=$ retrospective case series

$\mathrm{PCS}=$ prospective case series

Directness of evidence

Patients: = patients with SSD; defined as a sensorineural hearing loss (HL) with a threshold of $\geq 70 \mathrm{~dB}$ HL in the affected ear and a threshold of $\leq 30 \mathrm{~dB}$ $\mathrm{HL}$ in the better ear (pure tone average $0.5,1,2,4 \mathrm{kHz}$ ); $\mathrm{O}=$ other

Treatment: = cochlear implantation; $\bigcirc=$ other.

Outcome 1: = binaural hearing tests (speech perception in noise, sound localization); $\bigcirc=$ no information about binaural hearing provided.

Outcome 2: $\mathrm{O}$ = tinnitus; $\bigcirc=$ no information about tinnitus provided.

Outcome 3: $\bigcirc$ = quality of life; $\bigcirc=$ no information about quality of life provided.

Follow-up 1: $=\geq 6$ months; $\bigcirc=<6$ months

Follow-up 2: $=\geq 1$ year; $\bigcirc=<1$ year
Risk of bias

Treatment allocation: $=$ randomized or concealed; $\bigcirc$ = neither randomization nor concealment; ? = unclear, no information provided.

Blinding of intervention and interpretation of outcomes: - = patients and personnel blinded; $\bigcirc=$ only patients blinded or no blinding; ? = unclear, no information provided.

Standardization (T) of cochlear implantation (implant type and processor mentioned): $\bigcirc$ =yes; $\bigcirc=$ no; ? = unclear, no information provided.

Standardization $(\mathrm{O})$ of outcome measure: $\mathbf{O}=$ yes; $\bigcirc=$ no; ? = unclear, no information provided.

Completeness of outcome data for primary outcome: = below $15 \%$ missing data; $\bigcirc=15 \%$ or more missing data; ? = unclear, no information provided. 
Since none of the included studies was conducted as a randomized controlled trial (RCT) and none of the studies evaluated a control group, a low risk of bias was not assessed in one of the included articles. Blinding did not take place in any of the studies, since both professionals and patients are aware of cochlear implantation. Based on our criteria, three studies were considered as having a high risk of bias due to incomplete data and were excluded for further analysis. ${ }^{23,25,27}$ After the quality assessment, nine studies ( $\mathrm{n}=112$ patients) were left for further data extraction and analysis.

\section{Data extraction}

There is a large degree of clinical heterogeneity amongst the studies in terms of participants (classification of hearing loss [SSD versus AHL], duration of deafness and the indication for cochlear implantation [hearing loss versus tinnitus]), test conditions (CI-on versus CIoff and pre-implant versus post-implant), follow-up duration and outcome measurements (i.e. different test configurations, word tests and/or questionnaires) (Table 3). Owing to this heterogeneity, pooling of data was not possible. We therefore summarized the extracted data of the different outcomes (Tables 4 - 7). When studies did not report mean data or standard deviations in their text or tables, we did not derive them from graphs. Furthermore, in Tables 4- 7 several $p$-values are missing because they were not reported. 


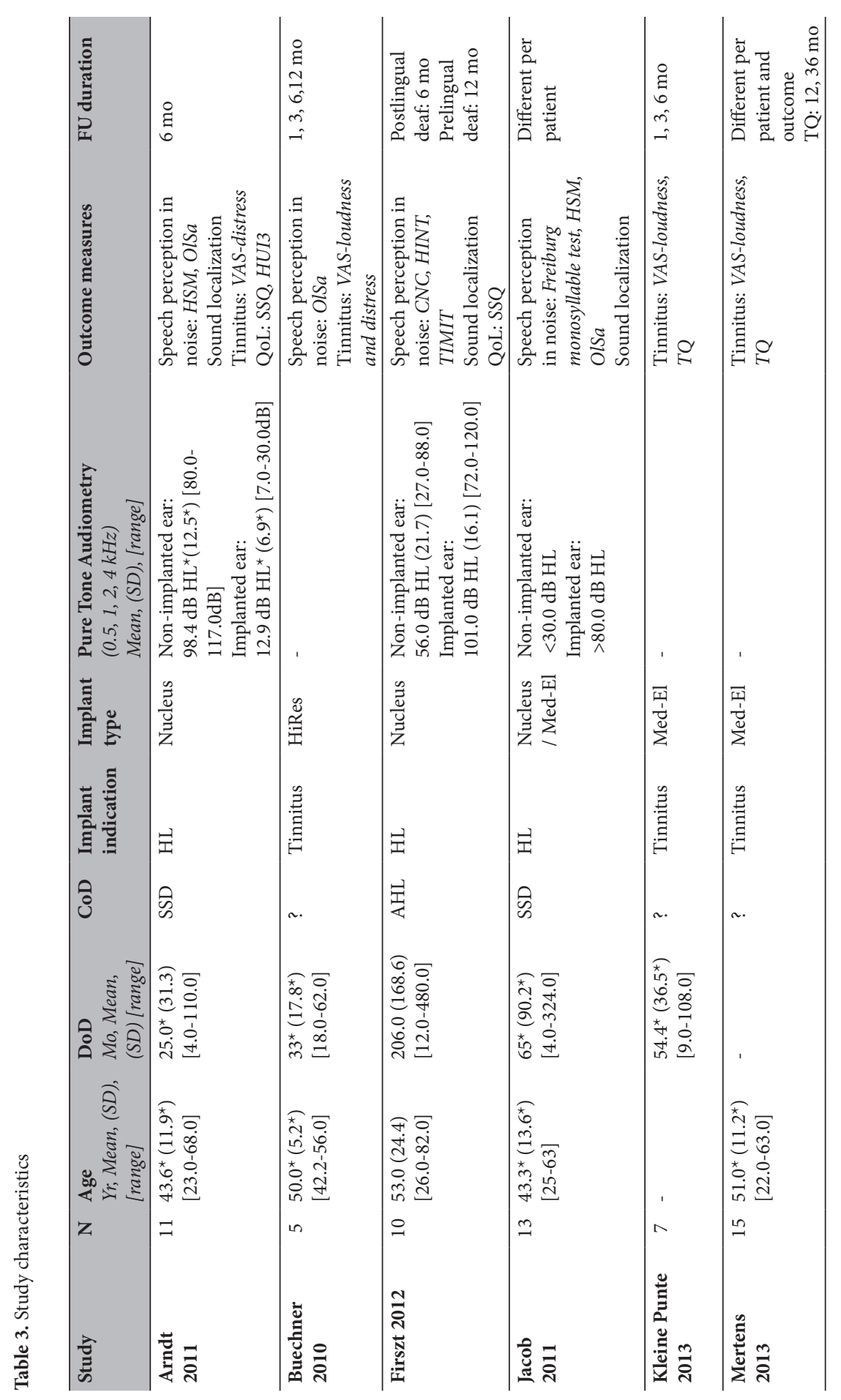




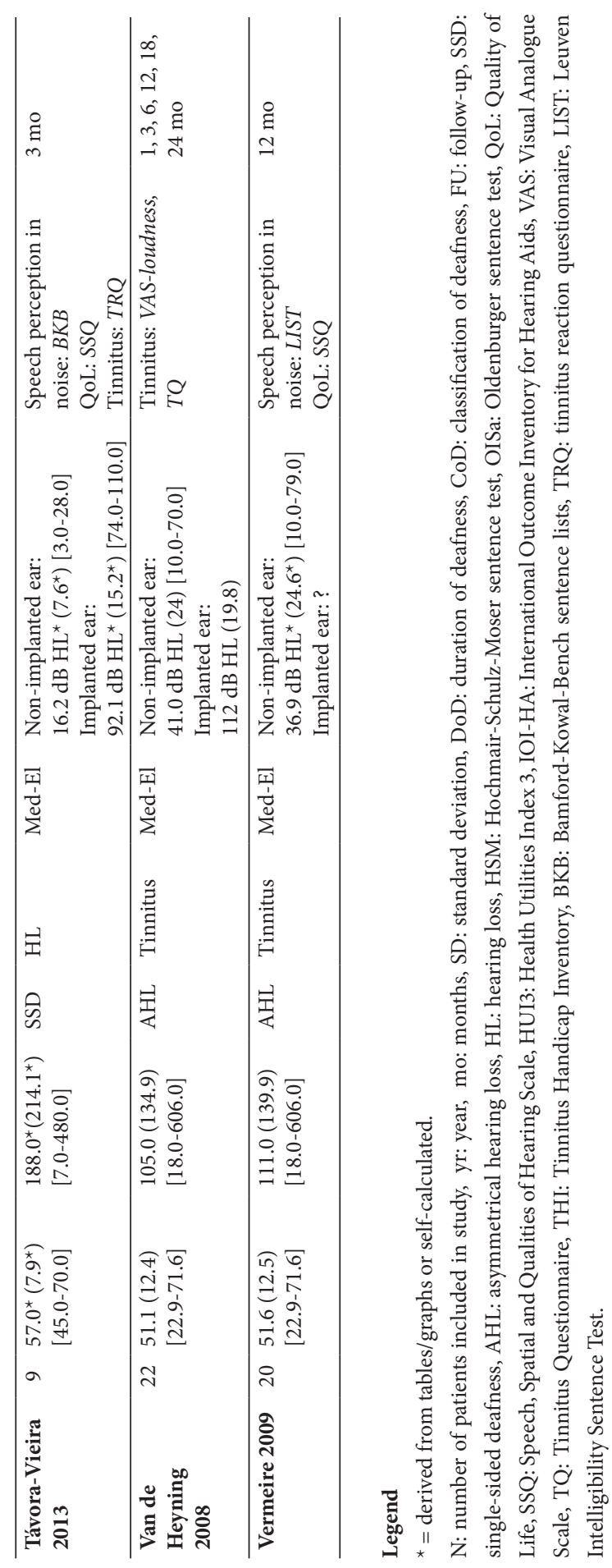




\section{Speech perception in noise}

Six studies reported on speech perception in noise $(n=68){ }^{28-33}$. In Table 4 the extracted data are summarized. Mertens et al. investigated the effect of a CI on tinnitus reduction ipsilaterally and improved speech perception in noise contralaterally and therefore their results are not taken into account in this section. ${ }^{34}$

Speech perception in noise can be measured using different configurations of spatially separated loudspeakers. We will indicate the configurations by abbreviating sound $(\mathrm{S})$ and noise $(\mathrm{N})$ followed by the direction; 'ci' for sound or noise coming from the CI side, 'be' for the 'better ear' side and finally ' 0 ' for 0 degrees azimuth.

Speech perception in noise was evaluated by five studies in terms of the signal-tonoise ratio $(\mathrm{dB})$, at which $50 \%$ of sentences is understood correctly. ${ }^{28,30-33}$ Both Arndt et al. and Távora-Vieira et al. demonstrated that sound perception in noise improved most in the SciNbe configuration. ${ }^{28,31}$ Also Vermeire et al. found a positive effect of CI activation, but significance of the results differed per subgroup; both the contralateral HA and normal-hearing $(\mathrm{NH})$ subgroups gain significant benefit from the CI in the SciN0 configuration, but only the HA subgroup also experienced significant benefit in the S0Nci configuration. ${ }^{32}$ In agreement with Arndt et al., they did not find significant differences in the S0N0 configuration. Jacob et al. did not find any changes in signal-to-noise ratios between the CI-on and CI-off conditions. ${ }^{30}$ Finally, Buechner et al. did not provide any numerical data, but stated that 3 out of 5 patients experienced a significant improvement in speech perception in noise. ${ }^{33}$

Three studies evaluated speech perception in noise as the total percentage of correctly repeated words. ${ }^{28-30}$ Arndt et al. found statistically significant improvement in the SciNbe configuration for the CI group compared to the other conditions, equivalent to their findings with the OlSa test. ${ }^{28}$ Interestingly, CI performance in the SbeNci configuration was superior to the scores in the CROS and BCD conditions, but not significantly different from the unaided group scores. For the prelingual onset of deafness group in the study of Firszt et al., no significant improvement was found between the hearing aid (HA) alone condition and 
the bimodal condition $(\mathrm{CI}+\mathrm{HA}) \cdot{ }^{29}$ For the postlingual deaf patients, there is an improvement after cochlear implantation, but they did not report significance on group level. The speech perception in noise scores in the study of Jacob et al. was not significantly different between the CI-on and CI-off conditions. ${ }^{30}$ 


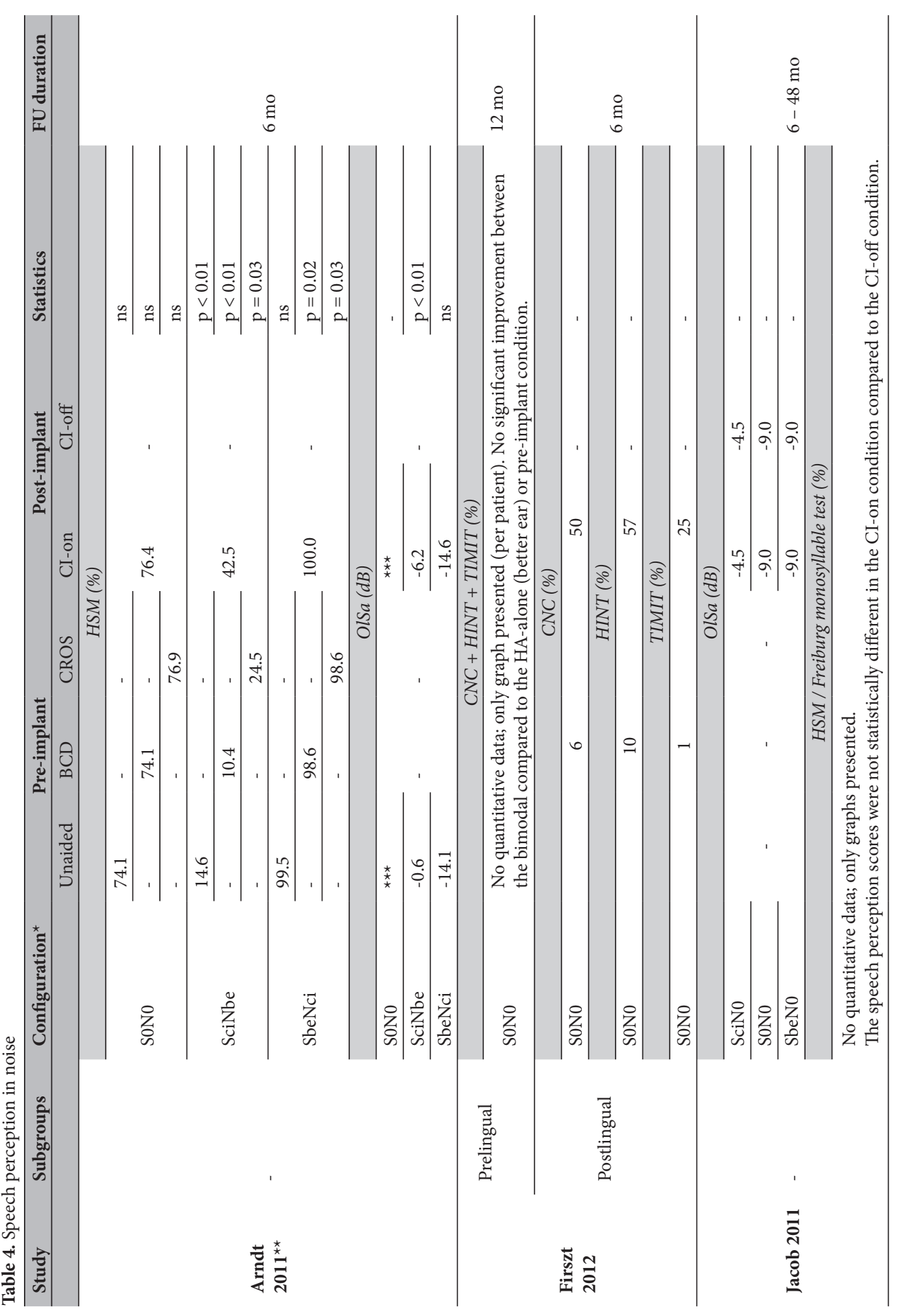




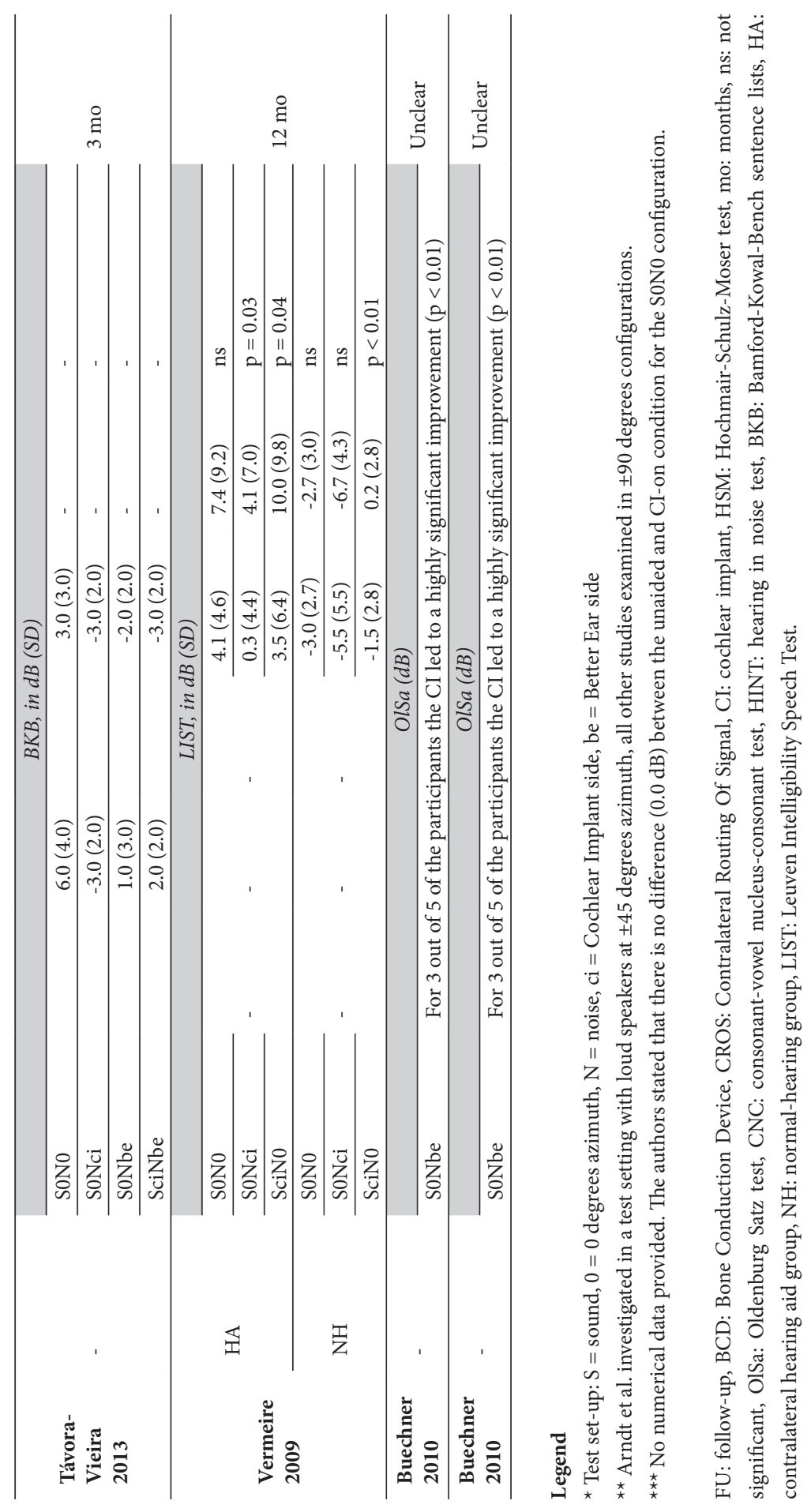




\section{Sound localization}

Three studies $(n=34)$ reported on sound localization. ${ }^{28-30}$ Data of the individual studies are presented in Table 5. Although the three studies used different test set-ups, they all used the localization error as outcome measure to assess localization. The localization error is the mean difference in degrees between the location of the sound source and the source indicated by the patient.

Arndt et al. found that the localization error reduced significantly after cochlear implantation compared to the pre-implant condition with either a CROS or BCD or the unaided condition. ${ }^{28}$ In the study of Jacob et al., participants were tested at different time points after cochlear implantation. They reported a reduction of the localization error from $48.0^{\circ}$ tot $4.0^{\circ}$ in the CI-on condition compared to the CI-off condition. However, no statistics were presented. ${ }^{30}$ Firszt et al. analyzed the data of postlingual deaf patients separately from patients with prelingual onset of deafness. ${ }^{29}$ They showed that the localization error reduced significantly in the bimodal (CI + HA) post-implant condition compared to hearing with the HA-alone (in better ear) in the postlingual deaf patients. This improvement was not found in the prelingual deaf patients. 
Table 5. Sound localization

\begin{tabular}{|c|c|c|c|c|c|c|c|c|c|}
\hline \multirow[t]{2}{*}{ Study } & \multirow[t]{2}{*}{ Test setup } & \multirow[t]{2}{*}{ Subgroup } & \multicolumn{3}{|c|}{ Pre-implant } & \multicolumn{2}{|c|}{ Post-implant } & \multirow[t]{2}{*}{ Statistics } & \multirow{2}{*}{$\begin{array}{l}\text { FU } \\
\text { duration }\end{array}$} \\
\hline & & & Unaided & $\mathrm{BCD}$ & CROS & CI-on & CI-off & & \\
\hline \multirow{3}{*}{$\begin{array}{l}\text { Arndt } \\
2011\end{array}$} & \multirow{3}{*}{$\begin{array}{l}7 \text { loudspeakers in } \\
\text { a } 180^{\circ} \text { arch with } \\
\text { intervals of } 30^{\circ} \text {. } \\
\text { Stimulus: OlSa } \\
\text { sentences (mean } \\
65 \mathrm{~dB} \text { ) }\end{array}$} & \multirow{3}{*}{-} & $33.9^{\circ}$ & - & - & \multirow{3}{*}{$15.0^{\circ}$} & \multirow{3}{*}{-} & $\mathrm{p}<0.01$ & \multirow{3}{*}{$6 \mathrm{mo}$} \\
\hline & & & - & $30.4^{\circ}$ & - & & & $\mathrm{p}<0.01$ & \\
\hline & & & - & - & $39.9^{\circ}$ & & & $\mathrm{p}<0.01$ & \\
\hline $\begin{array}{l}\text { Jacob } \\
2011\end{array}$ & $\begin{array}{l}11 \text { loudspeakers } \\
\text { in } 180^{\circ} \text { arch with } \\
\text { intervals of } 18^{\circ} \text {. } \\
\text { Stimulus: noise at } \\
\text { different loudness } \\
(60 \mathrm{~dB}, 70 \mathrm{~dB} \\
\text { and } 80 \mathrm{~dB})\end{array}$ & - & & - & & $4.0^{\circ}$ & $48.0^{\circ}$ & - & $\begin{array}{l}6-48 \text { mo } \\
\text { (differs per } \\
\text { patient) }\end{array}$ \\
\hline \multirow{2}{*}{$\begin{array}{l}\text { Firszt } \\
2012\end{array}$} & \multirow{2}{*}{$\begin{array}{l}15 \text { loudspeakers } \\
\text { in } 140^{\circ} \text { arch with } \\
\text { intervals of } 10^{\circ} \\
\text { Stimulus: } \\
\text { monosyllabic } \\
\text { words (mean } 60 \\
\text { dB) }\end{array}$} & Prelingual & \multicolumn{5}{|c|}{$\begin{array}{l}\text { No quantitative data; only graph (per patient) } \\
\text { presented. No significant improvement } \\
\text { reported after implantation in the bimodal } \\
\text { compared to the HA-alone (better ear) } \\
\text { condition. }\end{array}$} & - & $6 \mathrm{mo}$ \\
\hline & & Postlingual & \multicolumn{5}{|c|}{$\begin{array}{l}\text { No quantitative data; only graph presented. } \\
\text { The localization error after implantation } \\
\text { significantly reduced in the bimodal } \\
\text { compared to the HA-alone (better ear) } \\
\text { condition. }\end{array}$} & $\begin{array}{l}\mathrm{p} \leq 0.05 \\
\text { (for all } \\
\text { patients) }\end{array}$ & $6 \mathrm{mo}$ \\
\hline
\end{tabular}

\section{Legend}

FU: follow-up, BCD: Bone Conduction Device, CROS: Contralateral Routing Of Signal, CI: cochlear implant, mo: months, HA: hearing aid. 


\section{Quality of life}

QoL was reported in four studies $(n=50) \cdot{ }^{28,29,31,32}$ Data of the individual studies are presented in Table 6. All studies used the Speech, Spatial and Qualities of hearing (SSQ) questionnaire to assess QoL. The SSQ is divided into three subsections: 1) Speech, 2) Spatial and 3) Qualities of hearing.

In the study of Arndt et al. patients scored better on all sections of the SSQ in the condition with their CI compared to the three pre-implant conditions (unaided, BCD or CROS). ${ }^{28}$ The scores were significantly higher in the Speech and Spatial subsections. No significant differences were found in the Qualities of hearing subsection. Vermeire et al. showed a significant improvement after cochlear implantation compared to the pre-implant monaural condition in both the HA and NH group in the Speech and Qualities of hearing subsections and in the $\mathrm{NH}$ group also in the Spatial subsection. ${ }^{32}$ In the study of TávoraVieira, a significant improvement on all three subsections of the SSQ was demonstrated after cochlear implantation compared to the pre-implant condition. ${ }^{31}$ Firszt et al. showed a significant improvement pre- to post-implantation for the Speech and Spatial subsections in the postlingual deaf patients after 6 months and for the prelingual deaf patients only for the Spatial subsection after 12 months. ${ }^{29}$ No significant improvements were observed in the Qualities of hearing subsection.

One study evaluated QoL by means of the Health Utilities Index 3 (HUI3). ${ }^{28}$ They reported a significantly increased overall group score in the CI group compared to the pre-implant condition with either CROS or BCD. No significant improvement was found compared to the unaided condition. 
Table 6. Quality of Life

\begin{tabular}{|c|c|c|c|c|c|c|c|c|c|}
\hline \multirow[t]{2}{*}{ Study } & \multirow[t]{2}{*}{ Subgroups } & \multirow[t]{2}{*}{ Test } & \multirow[t]{2}{*}{ Subsection } & \multicolumn{3}{|c|}{$\begin{array}{c}\text { Pre-implant } \\
\text { (SD) }\end{array}$} & \multirow{2}{*}{$\begin{array}{c}\begin{array}{c}\text { Post- } \\
\text { implant } \\
\text { (SD) }\end{array} \\
\text { CI }\end{array}$} & \multirow[t]{2}{*}{ Statistics } & \multirow[t]{2}{*}{$\begin{array}{l}\text { FU } \\
\text { duration }\end{array}$} \\
\hline & & & & Unaided & $\mathrm{BCD}$ & CROS & & & \\
\hline \multirow{12}{*}{$\begin{array}{l}\text { Arndt } \\
2011\end{array}$} & \multirow[t]{12}{*}{-} & \multirow[t]{9}{*}{ SSQ } & \multirow{3}{*}{ Speech } & 2.6 & - & - & \multirow{3}{*}{5.8} & $\mathrm{p}=0.01$ & \\
\hline & & & & - & 2.9 & - & & $\mathrm{p}<0.01$ & \\
\hline & & & & - & - & 3.1 & & $\mathrm{p}=0.01$ & \\
\hline & & & \multirow{3}{*}{ Spatial } & 2.3 & - & - & \multirow{3}{*}{5.7} & $\mathrm{p}<0.01$ & \\
\hline & & & & - & 2.4 & - & & $\mathrm{p}<0.01$ & \\
\hline & & & & - & - & 2.6 & & $\mathrm{p}=0.03$ & \\
\hline & & & \multirow{3}{*}{ Qualities } & 5.9 & - & - & \multirow{3}{*}{7.8} & ns & $6 \mathrm{mo}$ \\
\hline & & & & - & 5.3 & - & & ns & \\
\hline & & & & - & - & 5.5 & & $\mathrm{~ns}$ & \\
\hline & & \multirow[t]{3}{*}{ HUI3 } & \multirow{3}{*}{ Overall } & 0.6 & - & - & \multirow{3}{*}{0.8} & ns & \\
\hline & & & & - & 0.7 & - & & $\mathrm{p}<0.01$ & \\
\hline & & & & - & - & 0.7 & & $\mathrm{p}<0.01$ & \\
\hline \multirow{6}{*}{$\begin{array}{l}\text { Vermeire } \\
2009\end{array}$} & \multirow{3}{*}{ HA } & \multirow[t]{3}{*}{ SSQ (SD) } & \multicolumn{2}{|l|}{ Speech } & \multicolumn{2}{|c|}{$2.1(1.2)$} & $4.3(1.5)$ & $\mathrm{p}=0.01$ & \multirow{6}{*}{$12 \mathrm{mo}$} \\
\hline & & & \multicolumn{2}{|l|}{ Spatial } & \multicolumn{2}{|c|}{$1.9(1.0)$} & $2.6(1.6)$ & ns & \\
\hline & & & \multicolumn{2}{|l|}{ Qualities } & \multicolumn{2}{|c|}{$3.5(1.7)$} & $5.8(2.2)$ & $\mathrm{p}<0.01$ & \\
\hline & \multirow[t]{3}{*}{$\mathrm{NH}$} & \multirow[t]{3}{*}{ SSQ (SD) } & Speech & & $3.9(1.4)$ & & $6.0(1.4)$ & $\mathrm{p}<0.01$ & \\
\hline & & & Spatial & & $3.0(1.5)$ & & $5.3(1.7)$ & $\mathrm{p}<0.01$ & \\
\hline & & & Qualities & & $5.8(1.5)$ & & $6.9(1.6)$ & $\mathrm{p}=0.05$ & \\
\hline Távora- & - & SSQ & Speech & No quar & titative & ata, only & graph & $\mathrm{p}<0.01$ & \\
\hline $\begin{array}{l}\text { Vieira } \\
2013\end{array}$ & & & Spatial & $\begin{array}{l}\text { present } \\
\text { significa }\end{array}$ & $\begin{array}{l}\text { d. They } \\
\text { nt impro }\end{array}$ & $\begin{array}{l}\text { eported } \\
\text { vement }\end{array}$ & r all three & $\mathrm{p}<0.01$ & $3 \mathrm{mo}$ \\
\hline & & & Qualities & subsecti & ons. & & & $\mathrm{p}<0.01$ & \\
\hline $\begin{array}{l}\text { Firszt } \\
2012\end{array}$ & Prelingual & SSQ & $\begin{array}{l}\text { No quantita } \\
\text { patient). } \\
\text { Significant b } \\
\text { the Spatial s }\end{array}$ & $\begin{array}{l}\text { tive data, } \\
\text { ubsefit fro } \\
\text { ubsection }\end{array}$ & $\begin{array}{l}\text { only grap } \\
\text { m pre- to }\end{array}$ & $\begin{array}{l}\text { h preser } \\
\text { post-im }\end{array}$ & $\begin{array}{l}\text { ed (per } \\
\text { lant on }\end{array}$ & - & $12 \mathrm{mo}$ \\
\hline & Postlingual & SSQ & $\begin{array}{l}\text { No quantita } \\
\text { Significant b } \\
\text { the Speech a }\end{array}$ & $\begin{array}{l}\text { tive data, } \\
\text { enefit fro } \\
\text { and Spatia }\end{array}$ & $\begin{array}{l}\text { only grap } \\
\text { m pre- to } \\
1 \text { subsect }\end{array}$ & $\begin{array}{l}\text { h preser } \\
\text { post-im } \\
\text { ons. }\end{array}$ & $\begin{array}{l}\text { ed. } \\
\text { blant on }\end{array}$ & - & $6 \mathrm{mo}$ \\
\hline
\end{tabular}

\section{Legend}

SD: standard deviation, FU: follow-up, BCD: Bone Conduction Device, CROS: Contralateral Routing Of Signal, CI: cochlear implant, mo: months, ns: not significant, NH: normal-hearing group, HA: contralateral hearing aid group, SSQ: Speech, Spatial and Qualities of Hearing questionnaire, HUI3: Health Utilities Index 3. 


\section{Tinnitus}

Tinnitus was reported in six studies $(\mathrm{n}=69) \cdot{ }^{10,28,31,33-35}$ Data of the individual studies are presented in Table 7. Several subjective scales were used to assess either tinnitus distress or tinnitus loudness and the included studies reported tinnitus at different time points after cochlear implantation.

Five studies used a Visual Analog Scale (VAS) to assess tinnitus. ${ }^{10,28,31,34,35}$ Three of them reported a significant reduction of the tinnitus distress or loudness after cochlear implantation..$^{10,28,34}$ The remaining studies also showed a reduction of tinnitus, but they did not present statistics. ${ }^{33,35}$ Furthermore, two studies showed that the tinnitus reoccurred after switching off the CI..$^{28,35}$

Three studies used the Tinnitus Questionnaire (TQ) to evaluate the effect of cochlear implantation on tinnitus..$^{10,34,35}$ Two of them reported a significant reduction of tinnitus ${ }^{10,35}$, Mertens et al. did not note significance. ${ }^{34}$

Only one study used the Tinnitus Reaction Questionnaire (TRQ) to assess tinnitus distress. $^{31}$ They reported a reduction of tinnitus varying from $77 \%$ to $100 \%$, but again, no statistics were presented. 
Table 7. Tinnitus

\begin{tabular}{|c|c|c|c|c|c|c|}
\hline \multirow[t]{2}{*}{ Study } & \multirow[t]{2}{*}{ Test } & \multirow{2}{*}{$\begin{array}{l}\text { Pre-implant } \\
\text { (SD) }\end{array}$} & \multicolumn{2}{|c|}{ Post-implant (SD) } & \multirow[t]{2}{*}{ Statistics } & \multirow{2}{*}{$\begin{array}{l}\text { FU } \\
\text { duration }\end{array}$} \\
\hline & & & CI-on & CI-off & & \\
\hline \multirow{2}{*}{$\begin{array}{l}\text { Arndt } \\
2011\end{array}$} & \multirow{2}{*}{ VAS distress } & \multirow{2}{*}{5.0} & 0.0 & - & $\mathrm{p}<0.01$ & \multirow{2}{*}{$6 \mathrm{mo}$} \\
\hline & & & - & 5.0 & - & \\
\hline \multirow{2}{*}{$\begin{array}{l}\text { Kleine Punte } \\
2013\end{array}$} & VAS loudness ${ }^{*}(\mathrm{SD})$ & $8.3(1.1)$ & $2.8(1.7)$ & $8.0(1.2)$ & - & \multirow{2}{*}{$6 \mathrm{mo}$} \\
\hline & TQ (SD) & $60.0(15.6)$ & $39.4(12.4)$ & - & $\mathrm{p}=0.04$ & \\
\hline \multirow{3}{*}{$\begin{array}{l}\text { Mertens } \\
2013\end{array}$} & VAS loudness (SD) & - & $3.4(2.5)$ & $7.2(2.6)$ & $\mathrm{p}<0.01$ & $12 \mathrm{mo}$ \\
\hline & \multirow{2}{*}{$\mathrm{TQ}(\mathrm{SD})$} & \multirow{2}{*}{$58.1(13.7)$} & $32.8(19.3)$ & - & - & $12 \mathrm{mo}$ \\
\hline & & & $26.3(20.0)$ & - & - & $36 \mathrm{mo}$ \\
\hline \multirow{13}{*}{$\begin{array}{l}\text { Van de } \\
\text { Heyning } \\
2008\end{array}$} & \multirow{7}{*}{ VAS loudness (SD) } & \multirow{7}{*}{$8.5(1.3)$} & $3.5(2.5)$ & - & $\mathrm{p}<0.01$ & \multirow{2}{*}{$1 \mathrm{mo}$} \\
\hline & & & - & $7.0(2.8)$ & $\mathrm{p}<0.01$ & \\
\hline & & & $2.2(2.0)$ & $6.6(3.0)$ & - & $3 \mathrm{mo}$ \\
\hline & & & $2.3(1.5)$ & $6.3(2.8)$ & - & $6 \mathrm{mo}$ \\
\hline & & & $2.4(1.8)$ & $6.6(2.6)$ & - & $12 \mathrm{mo}$ \\
\hline & & & $2.7(2.0)$ & $6.4(3.1)$ & - & $18 \mathrm{mo}$ \\
\hline & & & $2.5(1.9)$ & $6.1(2.9)$ & - & $24 \mathrm{mo}$ \\
\hline & \multirow{6}{*}{ TQ (SD) } & \multirow{6}{*}{$58.4(13.9)$} & $33.3(16.6)$ & - & $\mathrm{p}<0.01$ & $1 \mathrm{mo}$ \\
\hline & & & $32.4(19.9)$ & - & - & $3 \mathrm{mo}$ \\
\hline & & & $33.8(21.0)$ & - & - & $6 \mathrm{mo}$ \\
\hline & & & $34.3(20.1)$ & - & - & $12 \mathrm{mo}$ \\
\hline & & & $31.4(18.8)$ & - & - & $18 \mathrm{mo}$ \\
\hline & & & $38.9(19.4)$ & - & - & $24 \mathrm{mo}$ \\
\hline $\begin{array}{l}\text { Távora-Vieira } \\
2013\end{array}$ & TRQ distress & \multicolumn{3}{|c|}{$\begin{array}{l}\text { Significant decrease in TRQ score (range } \\
77.0-100.0 \% \text { ). }\end{array}$} & - & $3 \mathrm{mo}$ \\
\hline $\begin{array}{l}\text { Buechner } \\
2010\end{array}$ & $\begin{array}{l}\text { VAS distress and } \\
\text { loudness combined }\end{array}$ & \multicolumn{3}{|c|}{$\begin{array}{l}\text { Significant long-term decrease in VAS } \\
\text { score }(3 / 5 \text { patients, } 60 \%)) \text { and a significant } \\
\text { decrease in VAS score in certain situations } \\
(2 / 5 \text { patients, } 40 \%)) .\end{array}$} & - & unclear \\
\hline
\end{tabular}

\section{Legend}

SD: standard deviation, FU: follow-up, CI: cochlear implant, mo: months, VAS: Visual Analogue Scale,

TQ: Tinnitus Questionnaire, TRQ: Tinnitus Reaction Questionnaire.

* Discrepancy between results mentioned in the text and the tables. We adopted the results from the table. 


\section{DISCUSSION}

The major strength of this study is that we reviewed the effects of cochlear implantation on multiple clinical outcomes in patients with unilateral deafness systematically. To date, only non-systematic reviews have been conducted on this topic. ${ }^{36-38}$ Our systematic review is characterized by a transparent search strategy (Table 1), study selection and critical appraisal of selected studies. Also, we had strict criteria for including studies in our final data extraction, yielding better quality evidence. In addition to other reviews that only summarized data, we showed the results of individual studies in comprehensive tables per outcome.

One of the most important findings of this systematic review is that there are only non-randomized, low or moderate level of evidence studies on the topic of cochlear implantation for single-sided deafness. This needs to be taken into consideration when interpreting the described results.

Current review showed varying results for speech perception in noise after cochlear implantation compared to other modalities in patients with unilateral deafness. Although the success rates differed and the quality of the studies was suboptimal, modest improvements were observed. The largest significant improvement was reported when sound was presented to the CI side. ${ }^{28,32}$ All three studies that reported on sound localization showed a substantial improvement after cochlear implantation. ${ }^{28-30}$ Also QoL improved substantially after cochlear implantation when assessed with the SSQ, where most benefit was observed on the Speech subsection..$^{28,29,31,32}$ Our results on the previously described outcomes are generally congruent with the results of Kamal et al. and Vlastarakos et al. ${ }^{36,37}$

Historically, the initial intention of cochlear implantation in unilateral deaf patients was not the restoration of binaural hearing, but the treatment of tinnitus. All studies that focused on tinnitus in the current review objectified a substantial suppression of the complaints based on both the VAS and the TQ scores ${ }^{10,28,33-35}$ as by the TRQ scores ${ }^{31}$. None of the included studies reported tinnitus worsening after cochlear implantation. These results indicate that there is a positive effect of cochlear implantation on both tinnitus distress and 
loudness. Furthermore, we reported that two included studies showed that the tinnitus reoccurred after switching off the $\mathrm{CI}{ }^{28,35}$ This finding supports the paradigm that tinnitus results from cochlear deafferentiation. ${ }^{10}$ In 2012, Arts et al. reviewed the literature on cochlear implantation in patients with SSD to investigate the effect on tinnitus suppression. ${ }^{38}$ The results of their review and the review by Vlastarakos et al. are congruent with our results. ${ }^{37}$

Besides the lack of high quality evidence, there are some other limitations that we have to discuss. First, the sample sizes of the included studies are small. Two studies performed subgroup analysis which will eliminate statistical power when subgroups are already small. ${ }^{29,32}$

Next, there is a large degree of clinical heterogeneity amongst the studies that were included, which made it impossible to pool data. We will now summarize the most important differences in study characteristics: 1) the classification of deafness varies between and within study populations. ${ }^{10,29,32}$ The hearing thresholds for SSD and AHL are different, and therefore the affected contralateral ear in patients with AHL may have a negative impact on the hearing outcomes after cochlear implantation compared to patients with SSD;2) there is a large variation in the duration of deafness, again both between and within study populations. From studies in bilaterally deaf patients, it is known that, amongst other factors, the shorter the duration of deafness is, the better the CI performance..$^{39,40}$ In five of the studies that were included in this review the duration of deafness even surpassed ten years ${ }^{10,29-32} ; 3$ ) the indication for cochlear implantation differs amongst studies; 4) the onset of deafness varies within and between studies, or is not mentioned. Only Firszt et al. presented their results for patients with prelingual onset and postlingual deafness separately. ${ }^{29}$ They did not show benefit from cochlear implantation in patients with prelingual onset of deafness when sound localization and speech perception in noise were concerned. This finding highlights the possible limitation of cochlear implantation in prelingual deaf patients. However, Firszt et al. reported on only three patients with prelingual onset of deafness; 5) the follow-up time differed amongst and within studies, impeding comparison between studies. This may have influenced the results, since we know that the duration of cochlear implant use is an important 
predictor of speech perception ${ }^{40}$; 6) studies used different tests to assess their outcomes and different test conditions were used to examine the differences between monaural and binaural hearing. This counts most for speech perception in noise and may have led to the varying results for this outcome. In several studies, patients were asked to turn off their CI. The unilateral listening condition created in this manner cannot be compared to the preimplant condition.

Finally, there are no paediatric studies that passed our critical appraisal. Therefore no conclusions can be drawn about the effectiveness of cochlear implantation in children with unilateral hearing loss. Children with unilateral deafness are at increased risk of academic difficulties and behavioural issues. ${ }^{41}$ High quality research for this specific group should be conducted.

\section{CONCLUSION}

In conclusion, this systematic review shows that there are no high level of evidence studies concerning cochlear implantation in patients with SSD or AHL. Current literature suggests important benefits of cochlear implantation in this population regarding sound localization, QoL and tinnitus. Although results for speech perception in noise are promising as well, varying results between studies were reported for this outcome. This is possibly caused by the large clinical heterogeneity. Given the limited but promising results of cochlear implantation for patients with SSD or AHL, larger and high quality studies are certainly warranted before cochlear implantation can be considered as standard care. 


\section{REFERENCES}

1. Wie OB, Pripp HP, Tvete O. Unilateral Deafness in Adults: Effects on Communication and Social Interaction. The Annals of Otology, Rhinology, and Laryngology 2010;119(11):772-781.

2. Cox RM, DeChicchis AR, Wark J. Demonstration of Binaural Advantage in Audiometric Test Rooms. Ear \& Hearing 1981;2(5):194-201.

3. Bronkhorst AW, Plomp R. Binaural speech intelligibility in noise for hearing-impaired listeners. J Acoust Soc Am 1989;86(4):1374-1383.

4. Giolas T. Aural rehabilitation of adults with hearing impairment. In Katz J, ed: Handbook of Clinical Audiology, ed 4. Baltimore: Williams \& Wilkins, 1994;776-792.

5. Middlebrooks JC, Green DM. Sound localization by human listeners. Ann Rev Psychol 1991;42:135-159.

6. Akeroyd MA. The psychoacoustics of binaural hearing. Int J Audiol 2006;45:S25-S33.

7. Dunn CC, Tyler RS, Oakley S, Gantz BJ, Noble W. Comparison of Speech Recognition and Localization Performance in Bilateral and Unilateral Cochlear Implant Users Matched on Duration of Deafness and Age at Implantation. Ear \& Hearing 2008;29(3):325-359.

8. Van Wanrooij MM, Van Opstal AJ. Contribution of Head Shadow and Pinna Cues to Chronic Monaural Sound Localization. J Neuroscience 2004;24(17):4163-4171.

9. Van Schoonhoven J, Sparreboom M, Van Zanten GA, Scholten RJPM, Mylanus EAM, Dreschler WA, Grolman W, Maat B. The effectiveness of bilateral cochlear implants for severe-to-profound deafness in adults: a systematic review. Otology \& Neurotology 2013;34:190-198.

10. Van de Heyning P, Vermeire K, Diebl M, Nopp P, Anderson I, De Ridder D. Incapacitating unilateral tinnitus in single-sided deafness treated by cochlear implantation. The Annals of Otology, Rhinology, and Laryngology 2008;117(9):645-652.

11. Moher D, Liberati A, Tetzlaff J, Altman DG, The PRISMA Group. Preferred Reporting Items for Systematic Reviews and Meta-Analyses: The PRISMA Statement. BMJ 
2009;339:b2535.

12. Liberati A, Altman DG, Tetzlaff J, Mulrow C, Gøtsche PC, Ioannidis JPA, Clarke M, Deveraux PJ, Kleijnen J, Moher D. The PRISMA statement for reporting systematic reviews and meta-analyses of studies that evaluate healthcare interventions: explanation and elaboration. BMJ 2009;339:b2700.

13. Arndt S, Laszig R, Aschendorff A, Beck R, Schild C, Hassepass F, Ihorst G, Kroeger S, Kirchem P, Wesarg T. Unilateral deafness and cochlear implantation: audiological diagnostic evaluation and outcomes [in German]. HNO 2011;59(5):437-446.

14. Firszt JB, Holden LK, Reeder RM, Waltzman SB, Arndt S. Auditory abilities after cochlear implantation in adults with unilateral deafness: a pilot study. Otology \& Neurotology 2012;33(8):1339-1346

15. Vermeire K, Nobbe A, Schleich P, Nopp P, Voormolen MH, Van de Heyning P. Neural tonotopy in cochlear implants: an evaluation in unilateral cochlear implant patients with unilateral deafness and tinnitus. Hearing Research 2008;245(1-2):98-106.

16. Bernstein JG, Iyer N, Brungart DS. Spatial release from masking in simulations of cochlear implants for single-sided deafness. J Acoust Soc Am 2013;133(5):3383.

17. Song JJ, Punte AK, De Ridder D, Vanneste S, Van de Heyning P. Neural substrates predicting improvement of tinnitus after cochlear implantation in patients with singlesided deafness. Hearing Research 2013;299:1-9.

18. Boeheim K, Pok SM, Schloegel M. Audiologic evaluation after cochlear implantation in single sided deafness. Internat J Ped Otorhinolaryngo 2011;75(S1):21.

19. Kleine Punte A, Vermeire K, Hofkens A, De Bodt M, De Ridder D, Van de Heyning P. Cochlear implantation as a durable tinnitus treatment in single-sided deafness. Cochlear Implants International 2011;12(S1):S26-29.

20. Kleine Punte A, Vermeire K, Hofkens A, Van de Heyning P. Cochlear implantation in single-sided deafness improves spatial hearing and tinnitus. International Journal of Pediatric Otorhinolaryngology 2011;75:24. 
21. Roland JT Jr, Shapiro WH, Waltzman SB. Cochlear implantation as a treatment option for single-sided deafness: speech perception benefit. Audiol Neurotol 16(S1):8-9.

22. Hassepass F, Aschendorff A, Wesarg T, Kroger S, Laszig R, Beck RL, Schild C, Arndt S. Unilateral deafness in children: audiologic and subjective assessment of hearing ability after cochlear implantation. Otology \& Neurotology 2013;34(1):53-60.

23. Hassepass F, Schild C, Aschendorff A, Laszig R, Maier W, Beck R, Wesarg T, Arndt S. Clinical Outcome After Cochlear Implantation in Patients With Unilateral Hearing Loss Due to Labyrinthitis Ossificans. Otology \& Neurotology 2013:34;1278-1283.

24. Stelzig Y, Jacob R, Mueller J. Preliminary speech recognition results after cochlear implantation in patients with unilateral hearing loss: a case series. Journal of Medical Case Reports 2010;5:343.

25. Cadieux JH, Firszt JB, Reeder RM. Cochlear implantation in nontraditional candidates: preliminary results in adolescents with asymmetric hearing loss. Otology \& Neurotology, 2013;34(3):408-415.

26. Ramos A, Polo R, Masgoret E, Artiles O, Lisner I, Zaballos ML, Moreno C, Osorio A. Cochlear implant in patients with sudden unilateral sensorineural hearing loss and associated tinnitus. Acta Otorrinolaringologica Espanola 2012:63(1);15-20.

27. Hansen MR, Gantz, BJ, Dunn C. Outcomes After Cochlear Implantation for Patients With Single-Sided Deafness, Including Those With Recalcitrant Ménière's Disease. Otology \& Neurotology 2013:34;1681-1687.

28. Arndt S, Aschendorff A, Laszig R, Beck R, Schild C, Kroeger S, Ihorst G, Wesarg T. Comparison of pseudobinaural hearing to real binaural hearing rehabilitation after cochlear implantation in patients with unilateral deafness and tinnitus. Otology \& Neurotology 2011;32(1):39-47.

29. Firszt JB, Holden LK, Reeder RM, Cowdrey L, King S. Cochlear implantation in adults with asymmetric hearing loss. Ear \& Hearing 2012;33(4):521-533.

30. Jacob R, Stelzig Y, Nopp P, Schleich P. Audiological results with cochlear implants for 
single-sided deafness [in German]. HNO 2011;59(5):453-460.

31. Távora-Vieira D, Marino R, Krishnaswamy J, Kuthbutheen J, Rajan GP. Cochlear implantation for unilateral deafness with and without tinnitus: A case series. Laryngoscope 2013;123(5):1251-1255.

32. Vermeire K, Van de Heyning P. Binaural hearing after cochlear implantation in subjects with unilateral sensorineural deafness and tinnitus. Audiology \& Neurotology 2009;14(3):163-171.

33. Buechner A, Brendel M, Lesinski-Schiedat A, Wenzel G, Frohne-Buechner C, Jaeger B, Lenarz T. Cochlear implantation in unilateral deaf subjects associated with ipsilateral tinnitus. Otology \& Neurotology 2010;31(9):1381-1385.

34. Mertens G, Kleine Punte A, De Ridder D, Van de Heyning. Tinnitus in a single-sided deaf ear reduces speech reception in the nontinnitus ear. Otology \& Neurotology 2013;34(4):662-666.

35. Kleine Punte A, De Ridder D, Van de Heyning P. On the necessity of full length electrical cochlear stimulation to suppress severe tinnitus in single-sided deafness. Hearing Research 2013;295:24-29.

36. Kamal SM, Robinson AD, Diaz, RC. Cochlear implantation in single-sided deafness for enhancement of sound localization and speech perception. Curr Opin Otolaryngol Head Neck Surg 2012;20:393-397.

37. Vlastarakos PV, Nazos K, Tavoulari EF, Nikolopoulos TP. Cochlear implantation for single-sided deafness: the outcomes. An evidence based approach. Eur Arch Otorhinolaryngol 2014;271:2119-2126.

38. Arts RA, George EL, Stokroos RJ, Vermeire K. Review: cochlear implants as a treatment of tinnitus in single-sided deafness. Curr Opin Otolaryngol Head Neck Surg 2012;20(5):398-403.

39. United Kingdom Cochlear Implant Study Group. Criteria of Candidacy for Unilateral Cochlear Implantation in Postlingually Deafened Adults I: Theory and Measures of 
Effectiveness. Ear \& Hearing 2004;25:310-335.

40. Blamey P, Artieres F, Başkent D, Bergeron F, Beynon A, Burke E, Dillier N, Dowell R, Fraysse B, Gallégo S, Govaerts PJ, Green K, Huber AM, Kleine-Punte A, Maat B, Marx M, Mawman D, Mosnier I, O’Connor AF, O’Leary S, Rousset A, Schauwers K, Skarzynski H, Skarzynski PH, Sterkers O, Terranti A, Truy E, Van de Heyning P, Venail F, Vincent C, Lazard DS. Factors Affecting Auditory Performance of Postlinguistically Deaf Adults Using Cochlear Implants: An Update with 2251 Patients. Audiol Neurotol 2013;18:36-47.

41. Lieu JE. Speech-language and educational consequences of unilateral hearing loss in Children. Arch Otolaryng Head Neck Surg 2004;130:524-530. 


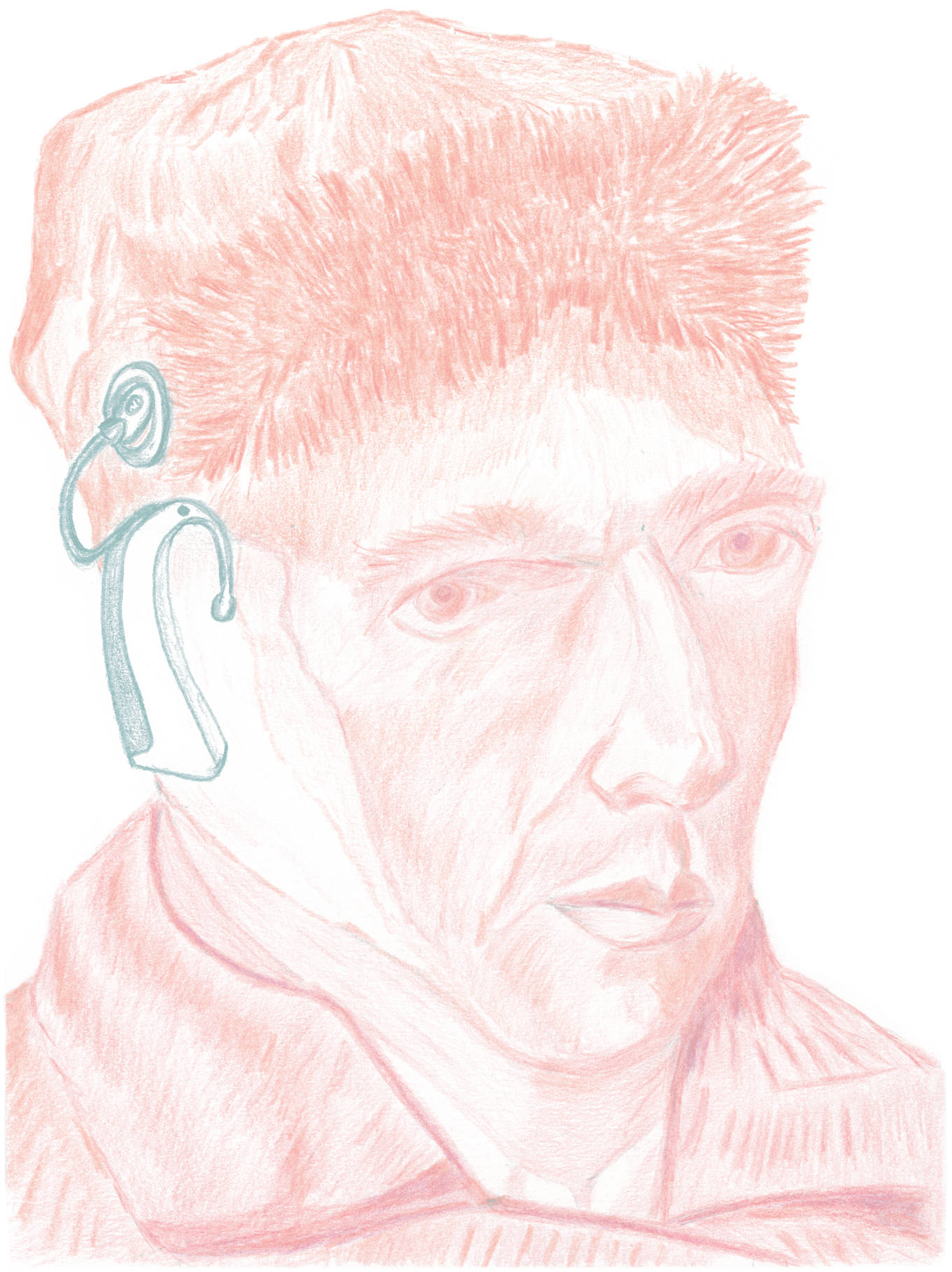




\section{Part II}

CINGLE-trial 


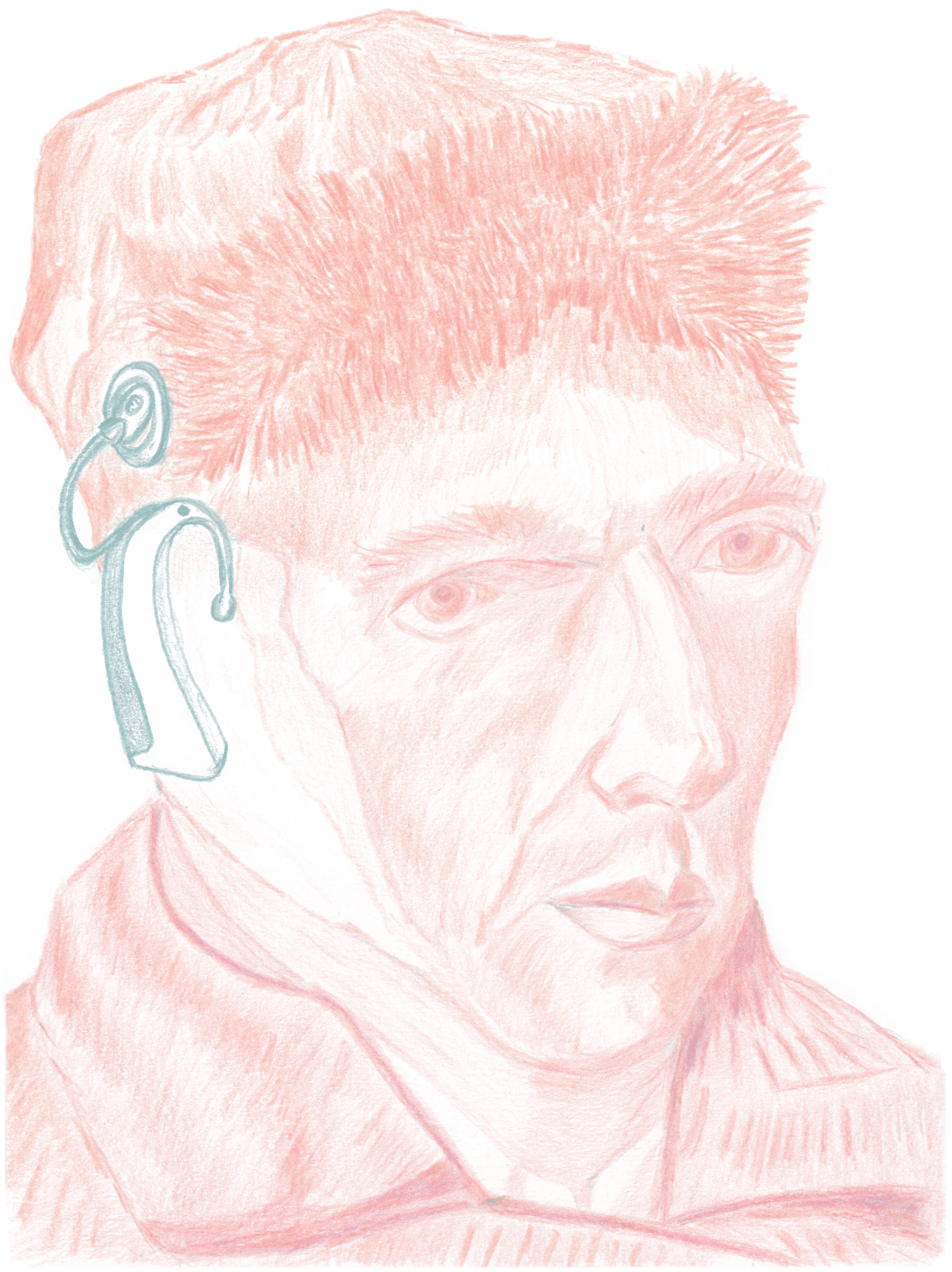




\section{4 \\ CINGLE-trial: \\ Cochlear Implantation for siNGLE-sided deafness, \\ a Randomised Controlled Trial and \\ Economic Evaluation}

Jeroen P.M. Peters, Alice van Zon, Adriana L. Smit, Gijsbert A. van Zanten,

G. Ardine de Wit, Inge Stegeman, Wilko Grolman

BMC Ear, Nose and Throat Disorders 2015;15:3 


\section{ABSTRACT}

\section{Background}

Individuals with single-sided deafness (SSD) have problems with speech perception in noise, localisation of sounds and with communication and social interaction in their daily life. Current treatment modalities (Contralateral Routing of Sound systems [CROS] and Bone Conduction Devices [BCD]) do not restore binaural hearing. Based on low level of evidence studies, CROS and BCD do not improve speech perception in noise or sound localisation. In contrast, cochlear implantation (CI) may overcome the limitations of CROS and $\mathrm{BCD}$, as binaural input can be restored. Promising results have previously been achieved on speech perception in noise, sound localisation, tinnitus and quality of life.

\section{Methods and design}

A single-center Randomised Controlled Trial (RCT) was designed to compare all treatment strategies for SSD. One hundred and twenty adult single-sided deaf patients (duration of deafness $>3$ months and maximum 10 years; pure tone average, at $0.5,1,2,4 \mathrm{kHz}$, of the deaf ear: threshold equal to or more than $70 \mathrm{~dB}$, of the better ear: threshold of maximum $30 \mathrm{~dB}$ ) will be included in this trial and randomised to treatment groups: $\mathrm{CI}$, trial periods 'first $\mathrm{BCD}$, then CROS' or 'first CROS, then BCD'. After the trial period, patients may choose with which treatment option they continue (BCD, CROS or no treatment). Outcomes of interest are speech perception in noise, sound localisation, tinnitus and quality of life. These outcomes will be measured during a baseline visit and at follow up visits, which will take place at 6,12 , $18,24,36,48$, and 60 months after onset of treatment. Furthermore, an economic evaluation will be performed and adverse events will be monitored.

\section{Discussion}

This RCT allows for a comparison between the two current treatment modalities for singlesided deafness and a new promising treatment strategy, CI, on a range of health outcomes: 
speech perception in noise, sound localisation, tinnitus and quality of life. Additionally, we will be able to answer the question if the additional costs of CI are justified by increased benefits, when compared to current treatment strategies. This study will inform health policy makers with regard to reimbursement of CI.

\section{Trial registration}

Netherlands Trial Register (www.trialregister.nl): NTR4580. 


\section{BACKGROUND}

Individuals with single-sided deafness (SSD) have problems with speech perception in noise and localisation of sound. ${ }^{1,2}$ With only one functional ear, they cannot benefit from binaural summation (redundancy of auditory input) ${ }^{3}$ and squelch effects (ability of the brain to separate sound and noise signals from spatially separated sources) ${ }^{1,2}$. Moreover, the head acts as an acoustic barrier and thus attenuates signals from the deaf side going to the better ear, known as the geometric head shadow effect. ${ }^{4}$ Patients suffering SSD experience problems in their daily life in social interaction and communication. ${ }^{5}$

Current treatment modalities for patients with SSD are Contralateral Routing of Sound (CROS) systems and Bone Conduction Devices (BCD). A CROS conducts signals from the hearing field of the poor ear via a wire (or FM/Bluetooth) to an output transducer in the ear canal of the better ear such that sound awareness is restored. A BCD transfers signals from the hearing field of the poor side to the better hearing ear by vibration of the skull bone via a titanium implant. A trial with a $\mathrm{BCD}$ can be performed by attaching the $\mathrm{BCD}$ to a tight headband. Theoretically, CROS and BCD can alleviate the head shadow effect. However, neither modality can restore binaural hearing. A recently published review found that there are no high level of evidence studies comparing CROS and BCD for single-sided deafness. ${ }^{6}$ The authors of the review could only include studies with low to moderate levels of evidence, and they found that CROS and BCD did not improve speech perception in noise or sound localisation, although patients did benefit in speech communication subjectively.

A new treatment option for single-sided deafness, cochlear implantation, may overcome the limitations of CROS and BCD. Since cochlear implantation restores auditory input on the impaired side, binaural input can be restored. Speech perception in noise and sound localisation improved in patients with single-sided deafness treated with a cochlear implant (CI). ${ }^{7-9}$ Furthermore, cochlear implantation may reduce tinnitus and improve quality of life. ${ }^{10}$ However, the quality of the studies included in these reviews was suboptimal: sample sizes were small, study designs were case series and they were prone to selection bias. ${ }^{11}$ 
The reviews on both current and new treatment options for single-sided deafness make clear that high level of evidence studies are warranted. Therefore, we initiated a Randomised Controlled Trial (RCT) comparing CROS, BCD, and CI for SSD. Outcomes of interest are speech perception in noise, sound localisation, quality of life and tinnitus. Finally, an economic evaluation will be performed. Since cochlear implantation is more expensive than current treatment options, it is important to know whether the promising results of cochlear implantation outweigh the additional costs.

\section{METHODS}

\section{Study objectives}

The main objective of our study is to compare CROS, BCD, and CI in patients with SSD evaluating speech perception in noise, sound localisation, tinnitus and quality of life. The second objective is to perform an economic evaluation and to evaluate adverse events in all groups.

\section{Study design}

In this RCT patients are randomised in three groups. For a schematic overview of the study, see Figure 1.

We will now discuss all consecutive steps in the study design as included in this study protocol. This protocol is reported according to the SPIRIT Statement, an international guideline on the reporting of study protocols. ${ }^{12}$ 
Figure 1. Flow diagram of CINGLE-trial

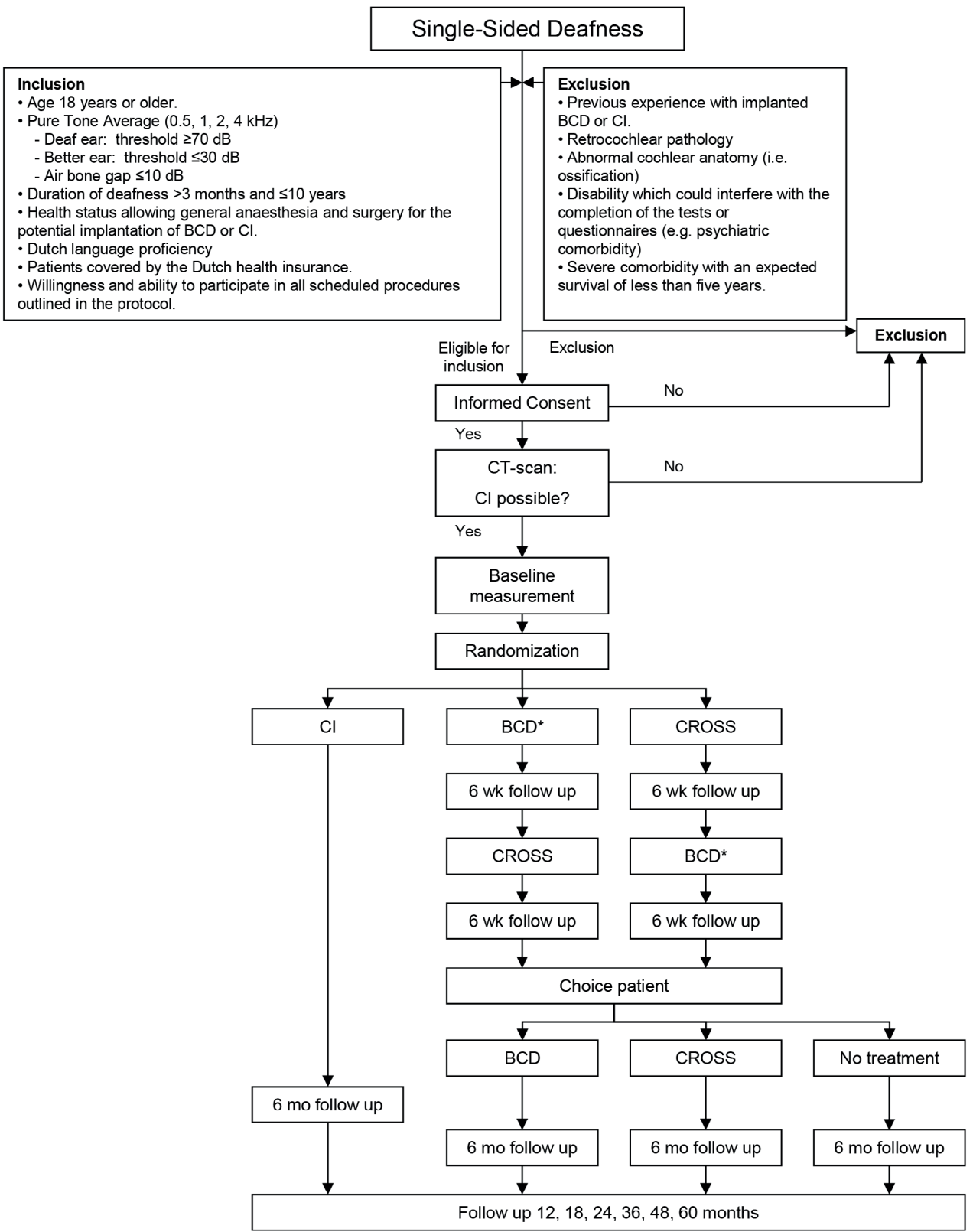

Legend:

Abbreviations: $\mathrm{BCD}=$ Bone Conduction Device $\left({ }^{*}\right.$ indicates trial period on headband), $\mathrm{CI}=$ Cochlear Implant, CROSS $=$ Contralateral Routing of Sound system, $\mathrm{mo}=$ months, $\mathrm{wk}=$ weeks 


\section{Study population}

The study population consists of patients who present with SSD at the outpatient department of our tertiary referral center, the University Medical Center Utrecht, Utrecht, The Netherlands. They must meet the following criteria to be eligible for the study.

\section{$\underline{\text { Inclusion criteria }}$}

- $\quad$ Age 18 years or older

- $\quad$ Audiometry (Pure Tone Average [PTA] at 0.5, 1, 2, $4 \mathrm{kHz}$ )

○ Deaf ear: threshold of $\geq 70 \mathrm{~dB}$

○ Better ear: threshold of $\leq 30 \mathrm{~dB}$

- Air bone gap $\leq 10 \mathrm{~dB}$ (to ensure normal middle ear function)

- $\quad$ Duration of deafness $>3$ months and $\leq 10$ years

- Health status allows general anaesthesia and surgery for the potential implantation of $\mathrm{BCD}$ or $\mathrm{CI}$

- $\quad$ Dutch language proficiency

- Coverage of Dutch health insurance

- Willingness and ability to participate in all scheduled procedures outlined in the protocol

The minimum duration of deafness is 3 months, since that is the time in clinical practice to await the natural course of sudden deafness. The maximum duration of deafness is up to 10 years, since degeneration of the auditory nerve may occur.

\section{Exclusion criteria}

- $\quad$ Previous experience with implanted BCD or CI

- Retrocochlear pathology

- Abnormal cochlear anatomy (i.e. ossification) 


\section{- Comorbidity}

- which could interfere with the completion of the tests or questionnaires (e.g. psychiatric)

$\circ \quad$ with an expected survival of less than five years

If eligible for inclusion, an Informed Consent (IC) form will be signed by patient and researcher. Only after IC, a CT-scan of the mastoid will be made, if none is available yet, to assess cochlear anatomy and check if no contraindications to cochlear implantation (e.g. ossification) exist. Since the anatomical situation must allow cochlear implantation (and thus randomisation), CT-scans will be performed prior to randomisation.

\section{Randomisation and Interventions}

A web-based randomisation tool (Julius Center, University Medical Center Utrecht, Utrecht, The Netherlands) will be accessed via a computer by one of the members of the research team. Patients will be randomised into groups A, B and C (see Figure 1), using a block size of 8 and a ratio of 2:3:3 for groups A:B:C and stratified for age ( $<45$ years, $\geq 45$ years). Blinding and concealed treatment allocation is not possible in our study design.

In group A, patients will be implanted with a CI from Cochlear Ltd., (type CI422). A retro-auricular incision is made to expose the mastoid. The electrode is inserted via a posterior tympanotomy and round window implantation. Intraoperatively, normal functioning of the device is checked by measurement of impedance and neural response telemetry. Four weeks after implantation, the CI will be activated by an experienced audiologist. In the rehabilitation phase, patients will be encouraged to use the CI each day and will be trained by experienced speech and language therapists. Patients are instructed to train the cochlear implant ear using an International Speech Test Signal (ISTS) noise ${ }^{13}$ via an insert earphone to mask the better ear.

In the Netherlands, a trial period with both CROS and BCD is standard clinical 
care. Patients in groups B and C try both devices for six weeks: group B starts with the BCD (type: BP110, Cochlear Ltd.), then CROS (Phonak Audeo Q50-312T and CROS $\mathrm{H}_{2} \mathrm{O}$ ), whereas patients in group C start with the CROS and then try the BCD. The reversed order is implemented to correct for the order effect: patients judge their second hearing aid based on experiences with the first hearing aid. After these two trial periods, patients may, according to clinical practice, choose which of both treatments they like best. When they choose a BCD, the implant and abutment will be surgically implanted, and after six weeks mounted with a BCD (type: BAHA 4 system, Cochlear Ltd.). When they prefer CROS, the patient is referred to standard clinical care where the CROS will be adjusted. Patients can also opt for no treatment if none is preferred, according to standard clinical health care.

\section{Sample size}

To detect a clinically relevant difference of $5 \mathrm{~dB}$ signal-to-noise ratio (SNR) (standard deviation $5 \mathrm{~dB}$ ) between the groups on the primary outcome (see Outcomes), with an alpha of 0.05 and a power of $95 \%, 27$ subjects per group are needed. To compensate for potential dropouts, a $10 \%$ margin is implemented, resulting in 30 patients in group A. Initial group distribution will be $\mathrm{n}=45$ for groups $\mathrm{B}$ and C. These groups are bigger, since previous studies described that only $45 \%$ of BCD-on-headband users are satisfied after their trial period and opt for a BCD implantation $(\mathrm{n} \sim 45) .{ }^{14}$ Therefore, we will include more patients in groups $\mathrm{B}$ and $\mathrm{C}$ than in group A. Patients not choosing a BCD can opt for a CROS (approximately $60 \%$ of remaining patients, $\mathrm{n} \sim 30$ ). The rest of the patients $(\mathrm{n} \sim 20)$ will probably prefer no treatment; they will be followed up to assess the natural course of single-sided deafness.

Approximately 25-30 patients per year present with SSD at the otorhinolaryngological outpatient department of our tertiary referral center. We will actively invite audiologic centers in the neighborhood to refer patients to our clinic. Therefore, we expect the inclusion period to last for $\sim 3$ years. 


\section{Outcomes}

The following outcome measurements will be recorded during baseline visit (one condition, i.e. 'no device') and follow-up visits at $6,12,18,24,36,48$, and 60 months (two conditions: 'device on' and 'device off'). During all audiometric tests, patients will be instructed not to move their heads to improve speech perception or sound localisation. Head movements will be checked by the researcher conducting the experiments. Patients will not receive feedback on their performance on audiometric tests. All experiments will be performed by researchers following the same protocol procedures.

\section{$\underline{\text { Primary outcome measure }}$}

Our primary outcome is the performance on speech perception in noise, measured with the Utrecht Sentence Test with Adaptive Randomized Roving Levels (U-STARR). ${ }^{15}$ In short, the U-STARR is designed to determine a patient's ability to understand speech in a noisy environment (signal from front, noise from front: $\mathrm{S}_{0} \mathrm{~N}_{0}$. See Figure 2, test set-up York Crescent of Sound ${ }^{16}$ ). A sentence is considered to be understood correctly when $\leq 2$ words are repeated incorrectly. The noise level starts at $+20 \mathrm{~dB}$ (roving 65-75 dB SPL). When the sentence is repeated correctly, the level of the noise increases for the next sentence. Noise is presented $500 \mathrm{~ms}$ before the start of the sentence and ends $500 \mathrm{~ms}$ after the sentence. Sentences used are traditional Dutch sentences from everyday life. ${ }^{17}$ The test provides a critical SNR at which $50 \%$ of sentences is understood correctly (in $\mathrm{dB}$ ). 
Figure 2: Set-up York Crescent of Sound

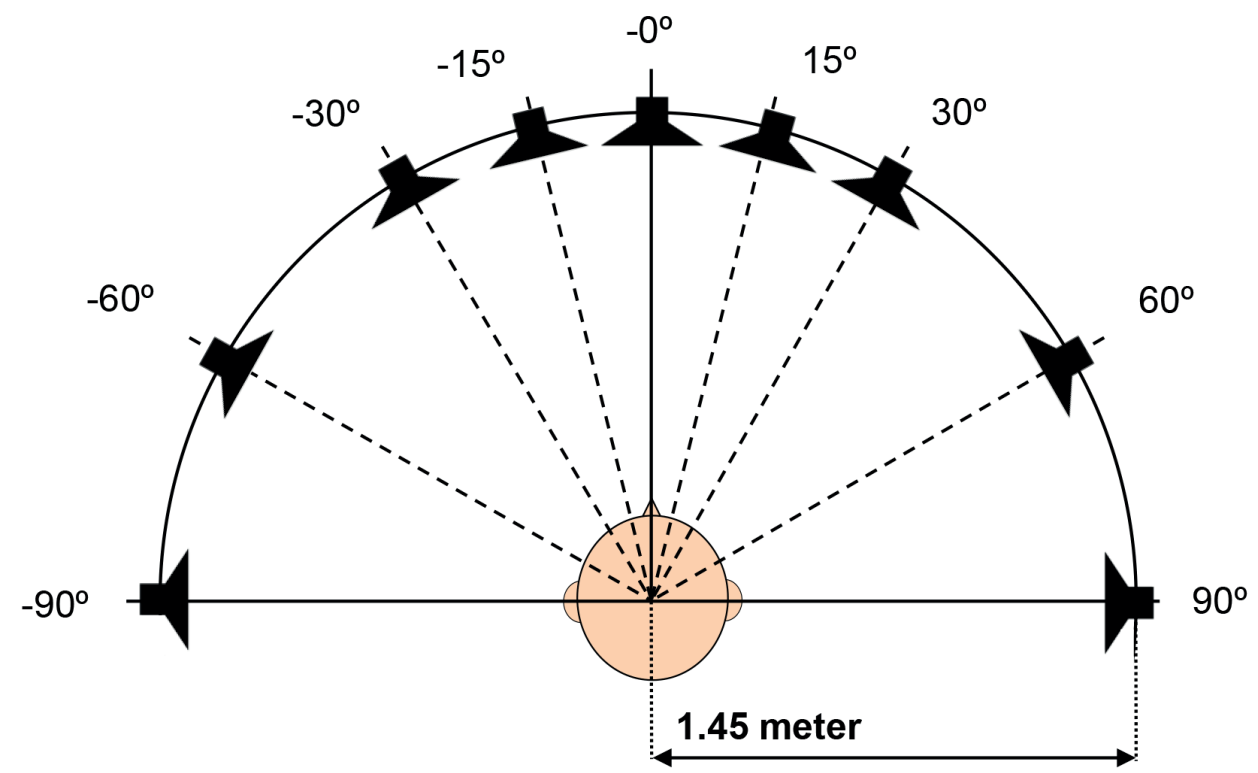

Legend: The patient is positioned in the center of an arch of loud speakers $\left(-90^{\circ}\right.$ to $\left.90^{\circ}\right)$ at head level with a radius of 1.45 meter.

\section{$\underline{\text { Secondary outcome measures }}$}

Speech perception in noise

Speech perception in noise is measured in two more configurations: $\mathrm{S}_{-60} \mathrm{~N}_{+60}$ and $\mathrm{S}_{+60} \mathrm{~N}_{-60}$

(Figure 2). Again, Dutch sentences are used in the same audiometric set-up as the U-STARR leading to a SNR (in $\mathrm{dB}$ ).

\section{Sound localisation}

The ability to localise sounds is also measured with the York Crescent of Sound set-up. ${ }^{16}$ The stimulus (sentence by female speaker: 'Hello, what's this?') is presented in quiet at a roving level of 55-65 dB SPL in three configurations: 5 boxes separated by an angle of $15^{\circ}$ (box $-30^{\circ}$, box $-15^{\circ}$, box $0^{\circ}$, box $+15^{\circ}$ and box $+30^{\circ}$ ), 5 boxes separated by an angle of $30^{\circ}$ (box $-60^{\circ}$, box $-30^{\circ}$, box $0^{\circ}$, box $+30^{\circ}$ and box $+60^{\circ}$ ) and 3 boxes separated by an angle of $60^{\circ}$ (box $-60^{\circ}$, box 
$0^{\circ}$ and box $+60^{\circ}$ ) (Figure 2). The patient must indicate from which box the stimulus came. The test outcome is a percent correct score.

\section{Tinnitus}

Tinnitus burden will be assessed using three questionnaires:

- Tinnitus Handicap Inventory (THI): a 25-item questionnaire with statements/ questions about tinnitus burden. ${ }^{18}$ Possible answers are 'Yes' (4 points), 'Sometimes' ( 2 points) and 'No' ( 0 points), resulting in a maximum score of 100 , representing a maximum burden of tinnitus. The inventory is divided in a functional, emotional and catastrophic subscale.

- Tinnitus Questionnaire (TQ): a 52-item questionnaire consisting of 5 subscales: emotional and cognitive distress, intrusiveness, auditory perceptual difficulties, sleep disturbances and somatic complaints. ${ }^{19,20}$ Possible answers are 'Yes' (2 points), 'Sometimes' ( 1 point) and 'No' ( 0 points). Of the 52 questions, 38 constitute a final TQ-score on the validated Dutch version of the TQ. ${ }^{21}$ Tinnitus can be graded mild (TQ 0-16), moderate (TQ 18-34), severe (TQ 34-56) and catastrophic (TQ >58).

- Tinnitus Burden Questionnaire (TBQ): this is a self-developed questionnaire assessing various aspects of tinnitus burden. It consists of 12 visual analogue scales (VAS), ranging from '0’ (no tinnitus burden) to '10' (maximum tinnitus burden).

\section{Quality of life}

Participants will be asked to fill in several questionnaires, each assessing different parts of QoL.

- $\quad$ Speech, Spatial and Qualities of hearing scale (SSQ): this questionnaire assesses three domains of hearing: 1) Speech: consists of 15 questions about the ability to separate speech from competing noise in a wide range of listening contexts; 2) Spatial: consists of 17 questions to assess the ability to locate sound sources and their 
direction of movement; 3) Qualities of hearing: consists of 19 questions that assess naturalness and clarity of sounds. The responses are given on a VAS ranging from 0 (not able to) to 100 (perfectly able to). ${ }^{22}$

- $\quad$ Abbreviated Profile for Hearing Aid Benefit (APHAB): this 24-item questionnaire documents the outcome of a hearing aid. ${ }^{23}$ The questionnaire yields scores on subscales: ease of communication, listening under reverberant conditions, listening in background noise and aversiveness of sounds.

- Glasgow Benefit Inventory (GBI): a measure of patient benefit developed especially for otorhinolaryngological interventions. ${ }^{24}$ The inventory is validated to measure outcomes on health status after otorhinolaryngological procedures. It measures QoL in three domains: social, general and physical. The domains score on a scale of -100 to 100 (minimum versus maximum benefit, respectively).

- Hospital Anxiety Depression Scale (HADS): a screening tool for anxiety and depression in non-psychiatric clinical populations. ${ }^{25}$ We use the HADS to measure baseline depression symptoms, which may confound/bias the results of, for instance, tinnitus burden.

- $\quad$ A VAS consisting of two questions (quality of life, quality of hearing).

- $\quad$ Time Trade Off (TTO): comprises one question about how many years of their lives patients would sacrifice for living with perfect hearing for the rest of their lives. TTO $(\%)=(($ life expectancy - number of years to give up for perfect hearing $) /$ life expectancy) ${ }^{*} 100$. This question is generally considered a difficult question, so it will not be presented on paper, but asked during the baseline and follow-up visits.

- $\quad$ EuroQoL5D (EQ5D): is a measure of general health status. ${ }^{26}$ It contains five questions on mobility, self-care, daily activities, pain/complaints, anxiety/depression and a scale to denote general quality of life (VAS 1-10). We will use the Dutch EQ5D tariff. $^{27}$

- Health Utilities Index 3 (HUI3): this is a generic QoL questionnaire consisting of 
eight domains: vision, hearing, speech, ambulation, dexterity, cognition, emotion and pain. ${ }^{28}$

\section{Economic evaluation}

The latter three questionnaires can be used to calculate utility. Utility reflects the value that is attached to health status. Utility values are important for the calculation of Quality Adjusted Life Years (QALYs), and serve as the denominator for the Incremental Cost Utility Ratio (ICUR). The ICUR is calculated as the incremental costs of cochlear implantation as compared to current treatments (numerator) divided by the incremental effects in terms of QALYs. Costs will be measured from a societal and health care perspective. Both direct health care costs and indirect non-health care costs will be incorporated in the analyses. Both categories of costs will be quantified using a cost diary. This diary is completed on a monthly basis the first two years and on a quarterly basis the last three years. This diary assesses costs related to hospitalisation, surgery, blood tests, complications (direct health care costs) and sick leave, time and travel costs (indirect health care costs). Unit prices for volumes of resources use will be taken from the Dutch guidelines for costing research in health economic evaluations, as issued by the National Healthcare Institute. ${ }^{29}$

Incremental Cost Utility Ratios, comparing CI with CROS and BCD, will be estimated using bootstrapping. Cost-effectiveness planes and a cost-effectiveness acceptability curve will be plotted to visually represent the results of the economic evaluation.

In addition to these outcome measurements at the previously specified baseline and follow-up visits, we will also objectify the experiences of the patients in Groups B and C in the trial periods with CROS and BCD (see Figure 1). To minimize patient burden, only the APHAB, GBI and SSQ questionnaires will be administered to evaluate these trial periods.

\section{Statistical analysis}

Baseline characteristics per group will be described as means and standard deviations. 
Differences between the three groups will be analysed using the Kruskal Wallis test.

The data of our primary outcome are quantitative and will be presented as continuous variables. Between-group mean differences, rate differences and rate ratios with 95\% confidence intervals will be calculated. Again, the Kruskal Wallis test will be used to analyse differences between the groups.

The secondary outcomes contain both categorical and continuous outcomes. Analyses of between-group differences will be performed with Chi-square-tests for categorical outcomes and Kruskal Wallis tests for continuous outcomes. Within-subject comparisons will entail differences of mean values. These will be analysed using paired t-tests for continuous measures.

Major test intervals are the same in all study groups (baseline and 6, 12, 18, 24, 36, 48, 60 months follow-up; 6 and 12 weeks for group B and C). Missing values will be imputed using multiple imputation. All analyses will be performed on an intention-to-treat basis. A significant result is defined as a $p$-value $<0.05$. Statistical package SPSS will be used for statistical analyses of the data.

Data will be presented according to the Consolidated Standards of Reporting Trials (CONSORT) Statement, an international guideline on adequate reporting RCTs. ${ }^{30,31}$

\section{Safety}

This study will be conducted in accordance with the most recent version of the Declaration of Helsinki (Fortaleza, 2013), good clinical practice guidelines and the Medical Research Involving Human Subjects Act of the Dutch government. The research protocol was approved by the Institutional Review Board (IRB) of the University Medical Center Utrecht (NL45288.041.13; version 3, April 2 $\left.{ }^{\text {nd }}, 2014\right)$.

All cases of serious adverse events will be reported to the local IRB and adequately followed up. An independent monitor (Trial Form Support BV, Zaltbommel, The Netherlands) is appointed to check trial quality (completeness of IC, validity of data etc.) twice a year. All 
patient data will be stored on a password protected computer in a lockable room. In the same room the signed Informed Consent forms will be kept in a locked cabin.

\section{DISCUSSION}

Patients suffering SSD experience problems with speech perception in noise and localisation of sound and in social interaction and communication. ${ }^{5}$

Current treatment modalities do not restore binaural hearing for patients with SSD. A recently published review concluded that there are no high quality studies comparing CROS and BCD for single-sided deafness. ${ }^{6}$ With this limited level of evidence, CROS and BCD did not improve speech perception in noise or sound localisation. Patients did benefit in speech communication subjectively. Cochlear implantation may overcome the limitations of CROS and $\mathrm{BCD}$, as binaural hearing can be restored. Promising results have previously been achieved on speech perception in noise, sound localisation, tinnitus and quality of life..$^{7-11}$

The current study is the first high level of evidence trial to be conducted to effectively compare all treatment strategies for SSD. One hundred and twenty adult single-sided deaf patients will be included in this trial and randomised to $\mathrm{CI}, \mathrm{BCD}-\mathrm{CROS}$ or CROS-BCD groups (Figure 1). Outcomes of interest are speech perception in noise, sound localisation, tinnitus and quality of life. Finally, an economic evaluation will be performed to answer the question if the additional costs of cochlear implantation are justified by increased benefits compared to current treatment strategies. 


\section{Author contributions}

All authors (JPMP, AvZ, ALS, GAvZ, GAdW, IS, WG) developed the protocol. JPMP and IS drafted the manuscript. All other authors revised the manuscript critically. All authors read and approved the final version of the manuscript.

\section{Competing Interests}

Wilko Grolman received unrestricted research grants from Cochlear Ltd., Med-El GmbH and Advanced Bionics. The authors have no other funding, financial relationships or conflicts of interest to declare.

\section{Funding}

This study was partly funded by Cochlear Ltd. as an unrestricted research grant. By research contract, Cochlear Ltd. did not have influence on the study design, data collection, analysis, data interpretation, and publication. 


\section{REFERENCES}

1. Akeroyd MA. The psychoacoustics of binaural hearing. Int J Audiol 2006:45;S25-S33.

2. Middlebrooks JC, Green DM. Sound localisation by human listeners. Ann Rev Psychol 1991:42;135-159.

3. Dunn CC, Tyler RS, Oakley S, Gantz BJ, Noble W. Comparison of speech recognition and localisation performance in bilateral and unilateral cochlear implant users matched on duration of deafness and age at implantation. Ear Hear 2008:29;325-359.

4. Van Wanrooij MM, Van Opstal AJ. Contribution of Head Shadow and Pinna Cues to Chronic Monaural Sound Localisation. J Neuroscience 2004: 24(17);4163-4171.

5. Wie OB, Pripp AH, Tvete O. Unilateral deafness in adults: effects on communication and social interaction. Annals of Otol Rhinol Laryngol 2010:119(11);772-781.

6. Peters JPM, Smit AL, Stegeman I, Grolman W. Review: Bone conduction devices and contralateral routing of sound systems in single-sided deafness. Laryngoscope 2015:125;218-226.

7. Kamal SM, Robinson AD, Diaz RC. Cochlear implantation in single-sided deafness for enhancement of sound localisation and speech perception. Curr Opin Otolaryngol Head Neck Surg 2012:20;393-397.

8. Vlastarakos PV, Nazos K, Tavoulari EF, Nikolopoulos TP. Cochlear implantation for single-sided deafness: the outcomes. An evidence based approach. Eur Arch Otorhinolaryngol 2014:271(8);2119-2126.

9. Blasco MA, Redleaf MI. Cochlear implantation in unilateral sudden deafness improves tinnitus and speech comprehension: meta-analysis and systematic review. Otol Neurotol 2014:35(8);1426-1432.

10. Arts RA, George EL, Stokroos RJ, Vermeire K. Review: cochlear implants as a treatment of tinnitus in single-sided deafness. Curr Opin Otolaryngol Head Neck Surg 2012:20(5);398-403.

11. Van Zon A, Peters JPM, Smit AL, Stegeman I, Grolman W. Cochlear Implantation for 
Patients with Single-Sided Deafness or Asymmetrical Hearing Loss: a Systematic Review of the Evidence. Otol Neurotol 2015:36;209-219.

12. Chan AW, TetzlaffJM, Altman DG, Laupacis A, Gøtzsche PC, Krleža-Jerić K, Hróbjartsson A, Mann H, Dickersin K, Berlin JA, Doré CJ, Parulekar WR, Summerskill WS, Groves T, Schulz KF, Sox HC, Rockhold FW, Rennie D, Moher D. SPIRIT 2013 statement: defining standard protocol items for clinical trials. Ann Intern Med 2013:158(3);200-207.

13. Holube I, Fredelake S, Vlaming M, Kollmeier B. Development and analysis of an International Speech Test Signal (ISTS). Int J Audiol 2010:49(12);891-903.

14. Desmet J, Bouzegta R, Hofkens A, De Backer A, Lambrechts P, Wouters K, Claes J, De Bodt M, Van de Heyning P. Clinical need for a Baha trial in patients with single-sided sensorineural deafness. Analysis of a Baha database of 196 patients. Eur Arch Otolaryngol 2012:269;799-605.

15. Smulders YE, Rinia AB, Pourier VEC, Van Zon A, Van Zanten GA, Stegeman I, Scherf WAC, Smit AL, Topsakal V, Tange RA, Grolman W. Validation of the U-STARR with the AB-York crescent of sound, a new instrument to evaluate speech intelligibility in noise and spatial hearing skills. Audiol Neurotol Extra 2015:5;1-10.

16. Kitterick PT, Lovett RE, Goman AM, Summerfield AQ. The AB-York crescent of sound: an apparatus for assessing spatial-listening skills in children and adults. Cochlear Implants Int 2011:12(3);164-169.

17. Versfeld NJ, Daalder L, Festen JM, Houtgast T. Method for the selection of sentence materials for efficient measurement of the speech reception threshold. J Acoust Soc Am 2000:107;1671-1684.

18. Newman CW, Jacobson GP, Spitzer JB. Development of the tinnitus handicap inventory. Arch Otolaryngol Head Neck Surg 1996:122;143-148.

19. Hallam RS, Jakes SC, Hinchcliffe R. Cognitive variables in tinnitus annoyance. Br J Clin Psychol 1988:27(pt3);213-222.

20. Goebel G, Hiller W. The tinnitus questionnaire. A standard instrument for grading the 
degree of tinnitus. Results of a multicenter study with the tinnitus questionnaire. HNO 1994:42(3);166-172.

21. Meeus O, Blaivie C, Van de Heyning P. Validation of the Dutch and the French version of the Tinnitus Questionnaire. B-ENT 2007:3(Suppl 7);11-17.

22. Gatehouse S, Noble W. The speech, spatial and qualities of hearing scale (SSQ). Inter J Audio 2004:43;85-99.

23. Cox RM, Alexander GC. The abbreviated profile for hearing aid benefit. Ear Hear 1998:16;176-186.

24. Robinson K, Gatehouse S, Browning GG. Measuring patient benefit from otorhinolaryngological surgery and therapy. Ann Otol Rhinol Laryngol 1996:105;415422.

25. Zigmond AS, Snaith RP: The hospital anxiety and depression scale. Acta Psychiatr Scand 1983:67;361-370.

26. The EuroQol Group: EuroQol - a new facility for the measurement of health-related quality of life. Health Policy 1990:16(3);199-208.

27. Lamers LM, Stalmeier PFM, McDonnell J, Krabbe PFM, Van Busschbach JJ. Kwaliteit van leven meten in economische evaluaties: het Nederlands EQ-5D-tarief. Ned Tijdsch v Geneesk 2005:149;1574-1578.

28. Feeny D, Furlong W, Torrance G. Multiple attributes and single attribute utility functions for Health Utility Index Mark 3 system. Medical Care 2002:40;113-128.

29. Hakkaart-van Roijen L, Tan SS, Bouwmans CAM. Handleiding voor kostenonderzoek, methoden en standaard kostprijzen voor economische evaluaties in de gezondheidszorg. College voor zorgverzekeringen. Geactualiseerde versie 2010. Available via www. zorginstituutnederland.nl, accessed November 10, 2014.

30. Schulz KF, Altman DG, Moher D, for the CONSORT group. CONSORT 2010 statement: updated guidelines for reporting parallel group randomised trials. BMJ 2010:340;c332.

31. Moher D, Hopewell S, Schulz KF, Montori V, Gøtzsche PC, Devereaux PJ, Elbourne 
D, Egger M, Altman DG, for the CONSORT group. CONSORT 2010 explanation and elaboration: updated guidelines for reporting parallel groups randomised trials. BMJ 2010:340;c869. 


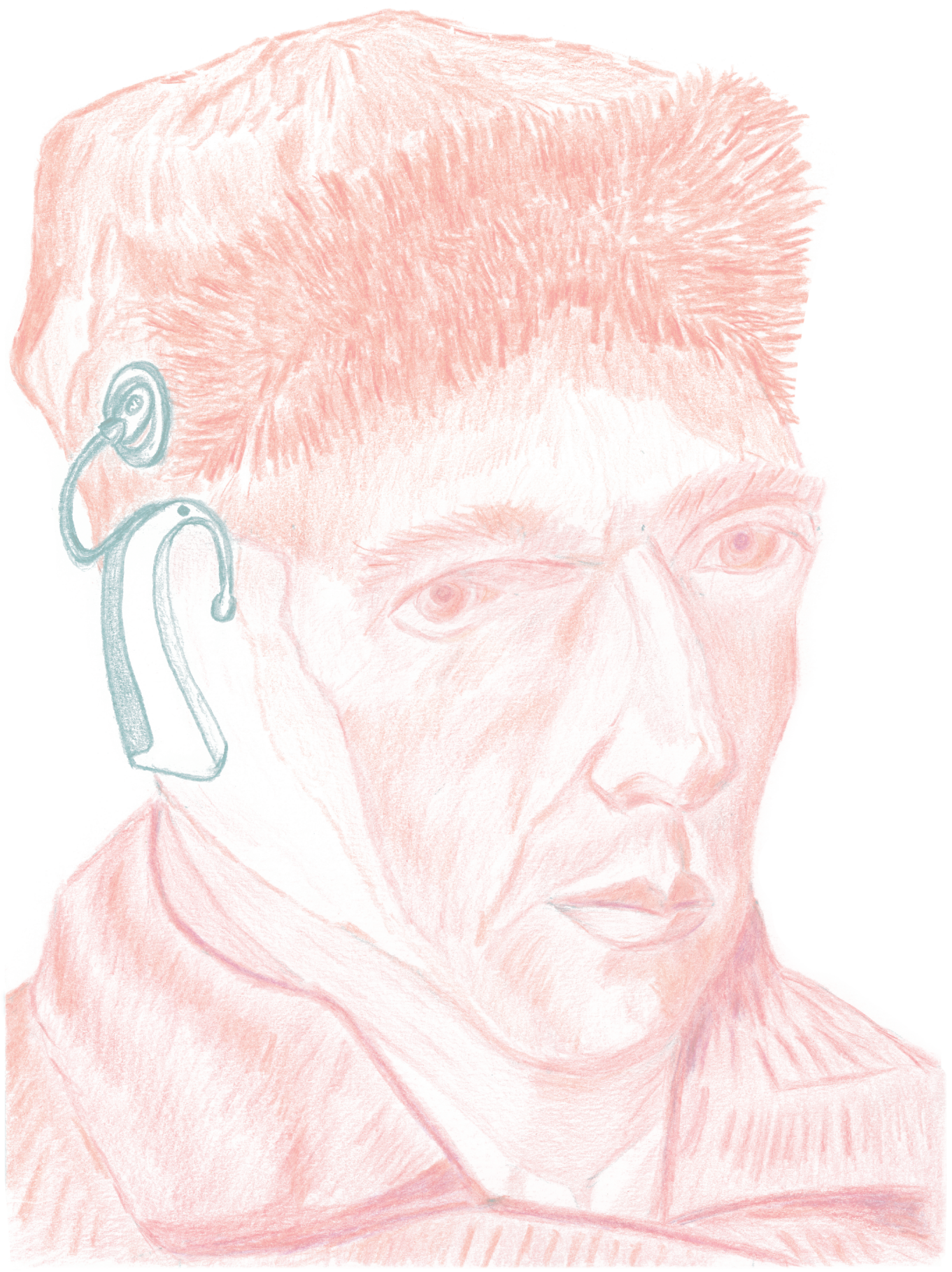




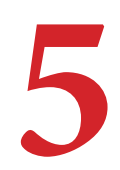

Short-term Outcomes of Cochlear Implantation for Single-Sided Deafness compared to Bone Conduction Devices and Contralateral Routing of Sound hearing aids - Results of a Randomised Controlled Trial (CINGLE-trial)

Jeroen P.M. Peters, Jan A.A. van Heteren*, Anne W. Wendrich*, Gijsbert A. van Zanten, Wilko Grolman, Robert J. Stokroos, Adriana L. Smit

${ }^{*}$ These authors contributed equally 


\section{ABSTRACT}

\section{Objectives}

Patients with single-sided deafness (SSD) often experience problems with speech perception in noise and sound localisation because they lack binaural benefits. Moreover, they frequently suffer from tinnitus and may have a lower quality of life (QoL). Current treatment modalities (Contralateral Routing of Sound hearing aids (CROS) and Bone Conduction Devices (BCD)) often do not sufficiently overcome these problems. Studies investigating cochlear implants (CIs) for SSD reported improved speech perception in noise, sound localisation and tinnitus suppression. However, only studies with considerable risk of bias have been published. Our aim was to evaluate CI, BCD, CROS, and no treatment for SSD in a Randomised Controlled Trial (RCT).

\section{Design}

Adult SSD patients (with a maximum pure tone average threshold of $30 \mathrm{~dB}$ HL in the better ear and a minimum threshold of $70 \mathrm{~dB}$ HL in the poor ear, and with a duration of deafness of 3 months - 10 years) were randomised using a web-based randomisation tool into one of three groups: 1) CI, 2) trial period of 'first BCD, then CROS', or 3) trial period of 'first CROS, then BCD'. After the trial period in groups 2 and 3, patients opted for BCD, CROS, or no treatment. The primary outcome was speech perception in noise (speech and noise directed from the front $\left(\mathrm{S}_{0} \mathrm{~N}_{0}\right)$ ). Secondary outcomes were speech perception in noise with speech directed to the poor ear and noise to the better ear $\left(\mathrm{S}_{\mathrm{pe}} \mathrm{N}_{\mathrm{be}}\right)$, and vice versa $\left(\mathrm{S}_{\mathrm{be}} \mathrm{N}_{\mathrm{pe}}\right)$, sound localization $\left(15^{\circ}, 30^{\circ}\right.$, and $60^{\circ}$ angle between loudspeakers), tinnitus burden measured with the Tinnitus Questionnaire (TQ) and the Tinnitus Handicap Inventory (THI), and disease-specific QoL (using the Speech, Spatial and Qualities of hearing subscale (SSQ), Abbreviated Profile for Hearing Aid Benefit (APHAB) and Glasgow Benefit Inventory (GBI)). We described results after 3 and 6 months of follow-up. 


\section{Results}

In total, 120 patients were randomised. The number of patients per group after allocation was: CI ( $n=28), \operatorname{BCD}(n=25)$, CROS $(n=34)$, and No treatment $(n=26)$. Seven patients did not receive the allocated intervention. In the $\mathrm{S}_{0} \mathrm{~N}_{0}$ configuration, the CI group performed significantly better than the other groups. In the $\mathrm{S}_{\mathrm{pe}} \mathrm{N}_{\text {be }}$ configuration there was an advantage for all treatment groups compared to baseline. However, in the $\mathrm{S}_{\mathrm{be}} \mathrm{N}_{\mathrm{pe}}$ configuration, $\mathrm{BCD}$ and CROS groups performed worse, whereas the CI group improved. Sound localisation improved in the CI group only. Patients with no tinnitus at baseline did not develop tinnitus after any intervention. After cochlear implantation, three patients reported total suppression of tinnitus. On both the TQ and THI, tinnitus burden decreased in the CI group. In general, all treatment groups improved on disease-specific QoL.

\section{Conclusions}

This RCT demonstrates that cochlear implantation leads to improved speech perception in noise, sound localization, tinnitus burden and QoL after 3 and 6 months of follow-up in adult patients with SSD. For most outcome measures, CI outperformed BCD and CROS. Results from long-term follow-up moments are warranted to evaluate long term performance and satisfaction.

Trial registration: Netherlands Trial Register (www.trialregister.nl): NTR4580, CINGLEtrial.

Level of Evidence: 1B. 


\section{INTRODUCTION}

\section{Single-sided deafness}

Single-sided deafness (SSD) is defined as severe to profound hearing loss in one ear and near to near-normal hearing in the contralateral ear. ${ }^{1}$ Patients with SSD often experience problems with speech perception in noise and sound localisation, because they lack binaural benefits. ${ }^{2}$ Specifically, they cannot benefit from the binaural summation effect (redundancy of auditory input) and the squelch effect (the ability of the brain to suppress noise on the side with the best signal-to-noise-ratio using the noise information of the contralateral side). ${ }^{2,3}$ Moreover, the head acts as an acoustic barrier and attenuates signals from the contralateral side (head shadow effect) ${ }^{2,4}$ Furthermore, SSD patients frequently suffer from tinnitus in the affected ear. ${ }^{2,5}$ Due to these hearing-related difficulties, patients with SSD may experience problems in social interaction and communication, possibly negatively affecting their quality of life (QoL). ${ }^{6-8}$

\section{CROS and BCDs}

In most countries, there are currently two reimbursed treatment options for patients with SSD: Contralateral Routing of Sound hearing aids (hereafter: CROS) and Bone Conduction Devices (BCDs). A CROS transfers signals from the hearing field of the poor ear to an output transducer in the ear canal of the better ear, so that awareness for sound at the poor side is restored. A BCD conducts signals from the hearing field of the poor side to the cochlea of the better ear by vibration of the skull bone via a titanium implant or tight headband. Theoretically, CROS and BCD can alleviate the head shadow effect. However, neither modality restores auditory input to the deaf ear.

So far, only retrospective case series comparing CROS and BCD for SSD have been published. ${ }^{9}$ These studies often had small sample sizes, unclear inclusion criteria and a moderate to high risk of bias. Conclusively, CROS and BCD did not lead to improved speech perception in noise or sound localisation, but patients did report to benefit in speech communication subjectively. 


\section{Cochlear implantation}

Since a cochlear implant (CI) provides input to the auditory nerve of the deaf ear, binaural input can be partially restored. Consequently, cochlear implantation led to improved speech perception in noise and sound localisation in patients with SSD. ${ }^{10-12}$

Tinnitus is frequently believed to be the consequence of (central) auditory deprivation due to hearing loss. Cochlear electrical stimulation of the auditory nerve is assumed to have a beneficial influence on tinnitus burden. ${ }^{13}$ Recent studies reported lower tinnitus burden following cochlear implantation in SSD patients. ${ }^{14-16}$ Moreover, (disease-specific) quality of life of SSD patients was also improved following cochlear implantation. ${ }^{11,15,17}$

However, all previously published studies on CI for SSD had methodological shortcomings that may have biased the results, e.g. retrospective study designs, unclear eligibility criteria, small sample sizes with lack of statistical power, or no power calculations. ${ }^{15,18,19}$

\section{Need for current trial}

With the identified lack of high-quality trials on treatment outcomes for SSD, we designed a Randomised Controlled Trial (RCT). In this paper we publish the short-term results of our RCT comparing CI, BCD, CROS, and no treatment for patients with SSD. The outcomes of interest were speech perception in noise, sound localisation, tinnitus burden, and QoL at 3 and 6 months follow-up (out of total follow-up duration of 5 years).

\section{MATERIALS AND METHODS}

The research protocol of this study was approved by the Institutional Review Board of the University Medical Center Utrecht (NL45288.041.13) and is registered in the Netherlands Trial Register (www.trialregister.nl, NTR4580). For a detailed description of the CINGLEtrial (Cochlear Implantation for siNGLE-sided deafness), we refer to the study protocol. ${ }^{20}$ In the current paper we presented a summary of the methods to assess the presented outcomes. This RCT is reported according to the CONSORT guidelines. ${ }^{21}$ 


\section{Study population}

Patients were eligible for inclusion if they fulfilled the following criteria:

1) age 18 years or older;

2) Pure Tone Average threshold at 0.5, 1, 2, $4 \mathrm{kHz}$ : of the best ear $\left(\mathrm{PTA}_{\mathrm{be}}\right)$ maximum $30 \mathrm{~dB} H \mathrm{HL}$, and of the poor ear minimum $70 \mathrm{~dB} \mathrm{HL}$;

3) duration of SSD minimum 3 months and maximum 10 years.

Patients were not eligible for inclusion if they had retrocochlear pathology or abnormal cochlear anatomy (e.g. ossification) or an implanted BCD.

\section{Study design}

Patients were randomised in one of three groups by a web-based randomisation tool (ratio 2:3:3, block randomisation):

- Group 1: CI (type: Cochlear ${ }^{\mathrm{TM}}$ )

- Group 2: trial period of first BCD on headband, then CROS

- Group 3: trial period of first CROS, then BCD on headband

In group 2 and 3, each device was tested for six weeks. After the trial period, patients were asked which of both treatments they preferred: when they opted for BCD, the abutment was surgically implanted and after six weeks mounted with a BCD $\left(\right.$ Cochlear $\left.^{\mathrm{Tm}}\right)$. Patients who opted for a CROS were referred to a local audiologist to purchase a CROS, and later device settings were optimized by an experienced audiologist from our audiological center. Patients could also opt for 'No treatment' if neither BCD nor CROS was preferred, resulting in four groups (CI, BCD, CROS, and No treatment).

In this paper, we report on data obtained at baseline and at 3 and 6 months followup. After approval of the study protocol ${ }^{20}$, but before the first patient was included in the trial, we amended the trial protocol to also measure at 3 months follow-up. 


\section{Outcomes}

\section{Speech perception in noise}

The primary outcome was speech perception in noise, with speech and noise coming front the front $\left(\mathrm{S}_{0} \mathrm{~N}_{0}\right)$ with the Utrecht Sentence Test with Adaptive Randomized Roving Levels (U-STARR). ${ }^{22}$ The U-STARR is designed to determine a patient's ability to understand speech in a noisy environment. Additionally, speech perception in noise was measured with speech directed to the poor ear (pe) and noise directed to the better ear (be) and vice versa $\left(\mathrm{S}_{\mathrm{pe}} \mathrm{N}_{\mathrm{be}}\right.$ and $\mathrm{S}_{\mathrm{be}} \mathrm{N}_{\mathrm{pe}}$, respectively), with the stimuli coming from $-60^{\circ}$ and $+60^{\circ}$. The outcome was a speech reception threshold in noise (SRTn) (in $\mathrm{dB}$ ) at which patients were able to repeat $50 \%$ of the sentences correctly.22

Speech perception in noise and sound localisation were measured in the Crescent of Sound test set-up ${ }^{23}$ by one of three coordinating researchers (JPMP, JAAvH, AWW) following the same protocol.

\section{$\underline{\text { Sound localisation }}$}

The stimulus (the sentence "hello, what's this?", spoken by various female English speakers) for the sound localisation task was presented in quiet at a roving level of $55-65 \mathrm{~dB}$ SPL in three configurations:

1) 5 loudspeakers separated by an angle of $15^{\circ}\left(-30^{\circ},-15^{\circ}, 0^{\circ},+15^{\circ}\right.$, and $\left.+30^{\circ}\right)$;

2) 5 loudspeakers separated by an angle of $30^{\circ}\left(-60^{\circ},-30^{\circ}, 0^{\circ},+30^{\circ}\right.$, and $\left.+60^{\circ}\right)$;

3) 3 loudspeakers separated by an angle of $60^{\circ}\left(-60^{\circ}, 0^{\circ}\right.$, and $\left.+60^{\circ}\right)$.

The patient had to indicate from which loudspeaker the stimulus came. The outcome was a percentage correct score. 


\section{$\underline{\text { Tinnitus }}$}

At baseline and at 3 and 6 months follow-up, patients indicated whether they had tinnitus or not. If yes, tinnitus questionnaires were completed. In this paper we report outcomes of the validated Dutch version ${ }^{24}$ of the Tinnitus Questionnaire (TQ) ${ }^{25}$ as a self-report measure of tinnitus-related distress and the Tinnitus Handicap Inventory (THI) ${ }^{26}$ that quantifies the impact of tinnitus on daily life.

\section{Disease-specific QoL}

Patients completed three disease-specific QoL questionnaires:

- $\quad$ The Speech, Spatial and Qualities of hearing (SSQ) scale ${ }^{27}$ assesses three domains of hearing in everyday life.

- $\quad$ The Abbreviated Profile for Hearing Aid Benefit (APHAB) questionnaire ${ }^{28}$ has four subdomains: ease of communication (EC), listening under reverberant conditions (RV), listening in background noise (BN), and aversiveness of sounds (AS).

- $\quad$ The Glasgow Benefit Inventory $(\mathrm{GBI})^{29}$ is validated to measure outcomes on health status after otorhinolaryngological procedures. The GBI is scored on three subscales (general, social support, and physical health).

\section{Statistical analysis}

We checked if the data were normally distributed with the Kolmogorov-Smirnov test, the Shapiro-Wilk test, and Q-Q-plots. Since none of the outcome variables were normally distributed, we used non-parametric tests. We used a generalized estimating equation (mixed model) to compare values within groups over time and between groups at follow-up moments. Due to the nature of our interventions, subject data were analysed 'as treated'. All figures show boxplots with medians, $1^{\text {st }}$ and $3^{\text {rd }}$ quartiles, and minimum/maximum values to show the distribution of data.

Because there was a low percentage of missing data for all outcomes, we performed 
a complete case analysis (missing data: speech perception in noise $0.9 \%$, sound localisation 0.9\%, TQ 3.5\%, THI 3.5\%, SSQ 1.2\%, APHAB 3.8\%, and GBI 1.9\%). A statistically significant result was defined as a $p$-value $<0.05$. Statistical package SPSS version 25.0 (IBM Corp., Armonk, NY, USA) was used.

\section{RESULTS}

\section{Patient characteristics}

We assessed 633 patients for eligibility. Of these, 513 patients were excluded due to various reasons (see Flow diagram in Figure 1). In total, 120 patients were randomised into three groups: 29 patients to the CI group, 45 patients to the 'first BCD, then CROS' group, and 46 patients to the 'first CROS, then BCD' group. All patients provided signed Informed Consent between July 2014 and February 2019. Not all patients received the allocated intervention; for an explanation of the numbers per group, see Supplementary Material 1. 


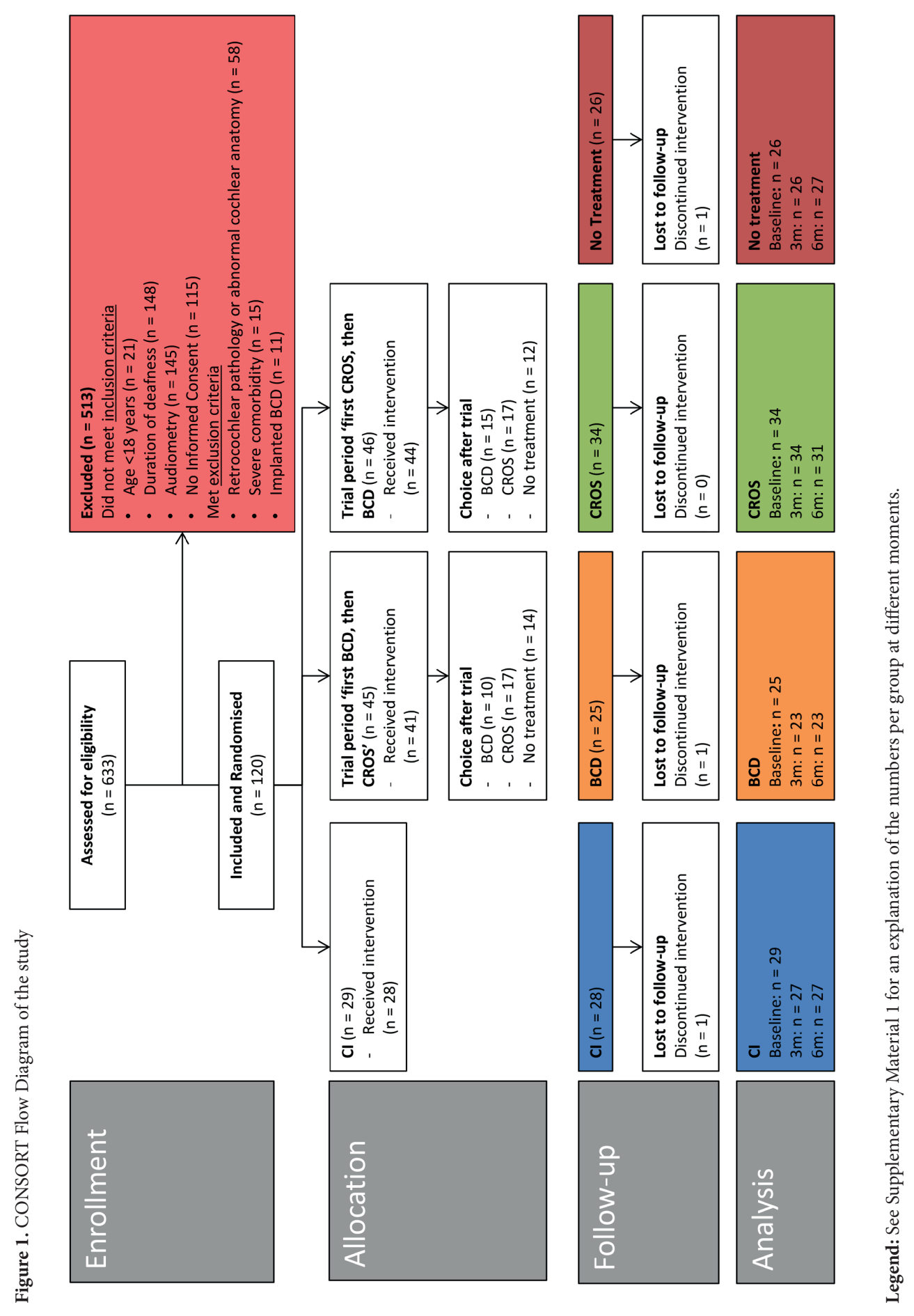


Table 1 shows the patient characteristics of our study population per group after randomisation. The mean age at inclusion of the complete study sample was $53.0 \pm 12.1$ (standard deviation, SD) years, the median PTA $_{\text {be }}$ was $15.0 \mathrm{~dB}$ (range 2.5 - $30.0 \mathrm{~dB}$ ) and the median duration of deafness was 1.8 years (range 3 months -10 years).

Table 1. Patient characteristics per group after randomisation

\begin{tabular}{|c|c|c|c|}
\hline & CI & BCD, CROS & CROS, BCD \\
\hline \multicolumn{4}{|l|}{ Gender } \\
\hline Male:Female & $14: 15$ & $24: 21$ & $20: 26$ \\
\hline \multicolumn{4}{|l|}{ Age at inclusion (years) } \\
\hline Mean (SD) & $53.2(13.1)$ & $53.6(12.6)$ & $52.3(11.2)$ \\
\hline \multicolumn{4}{|l|}{$\mathrm{PTA}_{\mathrm{be}}(0.5-4 \mathrm{kHz})(\mathrm{dB})$} \\
\hline Mean (SD) & $15.6(7.1)$ & $15.6(6.9)$ & $15.1(6.8)$ \\
\hline Median [range] & $15.0[5.0-30.0]$ & $15.0[6.3-30.0]$ & $15.6[2.5-28.8]$ \\
\hline \multicolumn{4}{|l|}{ Duration of deafness (years) } \\
\hline Mean (SD) & $3.1(2.8)$ & $3.1(3.3)$ & $2.7(2.7)$ \\
\hline Median [range] & $1.9[0.3-10.0]$ & $1.3[0.3-10.0]$ & $1.8[0.3-10.0]$ \\
\hline \multicolumn{4}{|l|}{ Etiology } \\
\hline Unknown & 6 & 10 & 13 \\
\hline Iatrogenic & 1 & 1 & 0 \\
\hline Sudden deafness & 15 & 21 & 23 \\
\hline Labyrinthitis & 4 & 4 & 4 \\
\hline Infection (not otherwise specified) & 0 & 3 & 1 \\
\hline Ménière's disease & 3 & 4 & 3 \\
\hline Traumatic & 0 & 2 & 2 \\
\hline
\end{tabular}

Abbreviations:

$\mathrm{CI}=$ Cochlear Implant, BCD = Bone Conduction Device, CROS $=$ Contralateral Routing of Sound hearing aid, SD $=$ standard deviation, $\mathrm{PTA}_{\mathrm{be}}=$ Pure Tone Average threshold $(\mathrm{dB})$ of the better ear at $0.5,1,2,4 \mathrm{kHz}$. 
The patient characteristics of the groups after allocation of treatment (i.e. CI activation, BCD implantation and CROS fitting) and for the No treatment group are displayed in Supplementary Material 2. There were no significant differences in patient characteristics between these groups $(p>0.05)$.

\section{Device characteristics}

Due to the long inclusion period of the study, newer versions of the CI and BCD became available during the study. The first 12 CI patients were implanted with a Nucleus CI422 with a Slim Straight electrode array, the other 16 patients were implanted with a Nucleus ${ }^{\circ}$ CI512 with a Contour Advance electrode array. The median duration of CI use per day during the first 6 months after fitting was 12.7 hours (range 2.0 - 16.3 hours), based on the electronic data logging of the speech processor. There were no non-users in the CI group.

In the trial period, 52 patients tested the Baha BP110 on a headband from 2014 until 2016. From 2017 on, 33 patients tested the Baha 5 Power on a headband. There was a remarkable difference in satisfaction after the trial period with these two devices: 10 of 52 patients $(19.2 \%)$ chose to proceed with BCD after the Baha $\mathrm{BP} 110$ trial, whereas 15 of 33 patients (45.5\%) opted for BCD after the Baha 5 Power in the trial period (Fisher's exact test, $p=0.014)$. Thus, in total 25 of 85 patients $(29.4 \%)$ opted to be implanted with a BCD after the trial period. After implantation, three patients were mounted with the Baha BP110 and all other patients were mounted with the Baha 5 Power. 


\section{Speech perception in noise}

The results on speech perception in noise are shown in Figure 2a-c. Lower values (SRTn in dB) reflect better performance. See Supplementary Material 3 for exact data.

\section{$S_{0} N_{0}$}

For the CI group, there was a statistically significant improvement in speech perception in noise (with speech and noise coming from the front) at 3 and 6 months follow-up compared to baseline (Figure 2a). Also the BCD group had a significant improvement compared to baseline, but only at 6 months follow-up. Even when one outlier was not included in the analysis, these results remained statistically significant. There were no significant changes for the CROS and No treatment groups.

At 3 months follow-up, the CI group performed better than all other groups. At 6 months, the CI group performed significantly better than the BCD and No treatment groups. The difference between the CI group and the CROS group was not statistically significant. 
Figure 2. Speech perception in noise

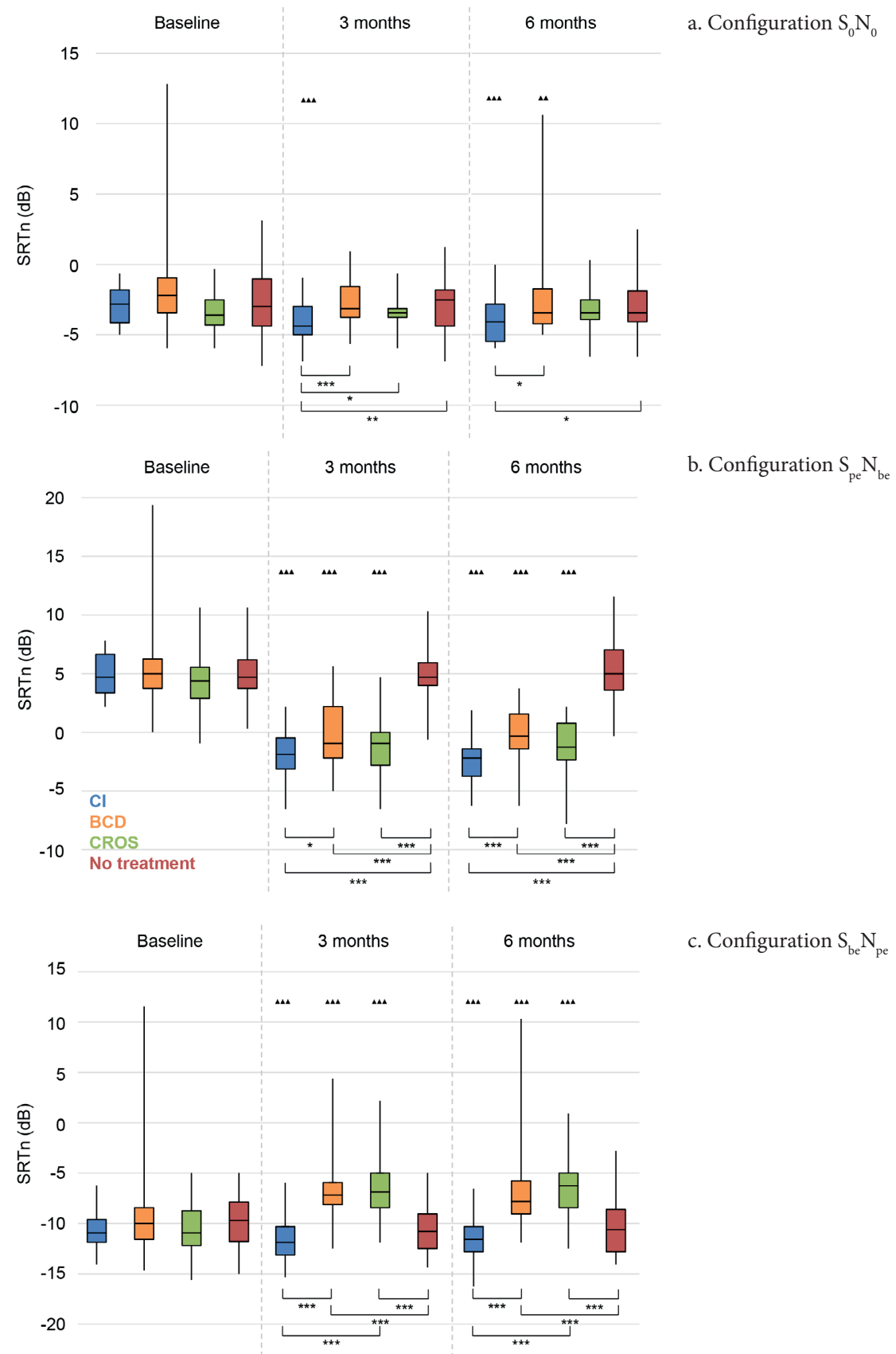


Legend: Speech reception threshold in noise (SRTn) in decibels (dB) per configuration.

Statistical difference compared to baseline

$\boldsymbol{\Delta} \boldsymbol{\Delta} \boldsymbol{\Delta}=p<0.001 ; \boldsymbol{\Delta} \boldsymbol{\Delta}=p<0.01 ; \boldsymbol{\Delta}=p<0.05$

Statistical difference compared to 3 months follow-up

-" = $p<0.001 ; \boldsymbol{\omega}=p<0.01 ; \boldsymbol{- =} p<0.05$

Statistical difference between groups at same follow-up moment

${ }^{* * *}=p<0.001 ;{ }^{* *}=p<0.01 ;{ }^{*}=p<0.05$

These symbols display the significance of the results, and are also used in the other figures in this chapter.

$S_{p e} N_{b e}$

With speech directed to the poor ear, all treatment groups (CI, BCD, CROS) showed statistically significantly improved speech perception at 3 and 6 months follow-up compared to baseline (Figure 2b). There was no significant change for the No treatment group during follow-up.

At 3 and 6 months follow-up, the patients in all treatment groups had better speech perception in noise than the patients in the No treatment group. The CI group also performed statistically significantly better than the BCD group.

$S_{b e} N_{p e}$

With speech directed to the better ear, the CI group showed a significant improvement in speech perception at 3 and 6 months follow-up compared to baseline, whereas both the BCD and CROS group deteriorated significantly (Figure 2c). The No treatment group showed no significant changes over time.

At 3 and 6 months follow-up, the BCD and CROS group had a significantly worse speech perception than the CI and No treatment groups. 
Figure 3. Sound localisation
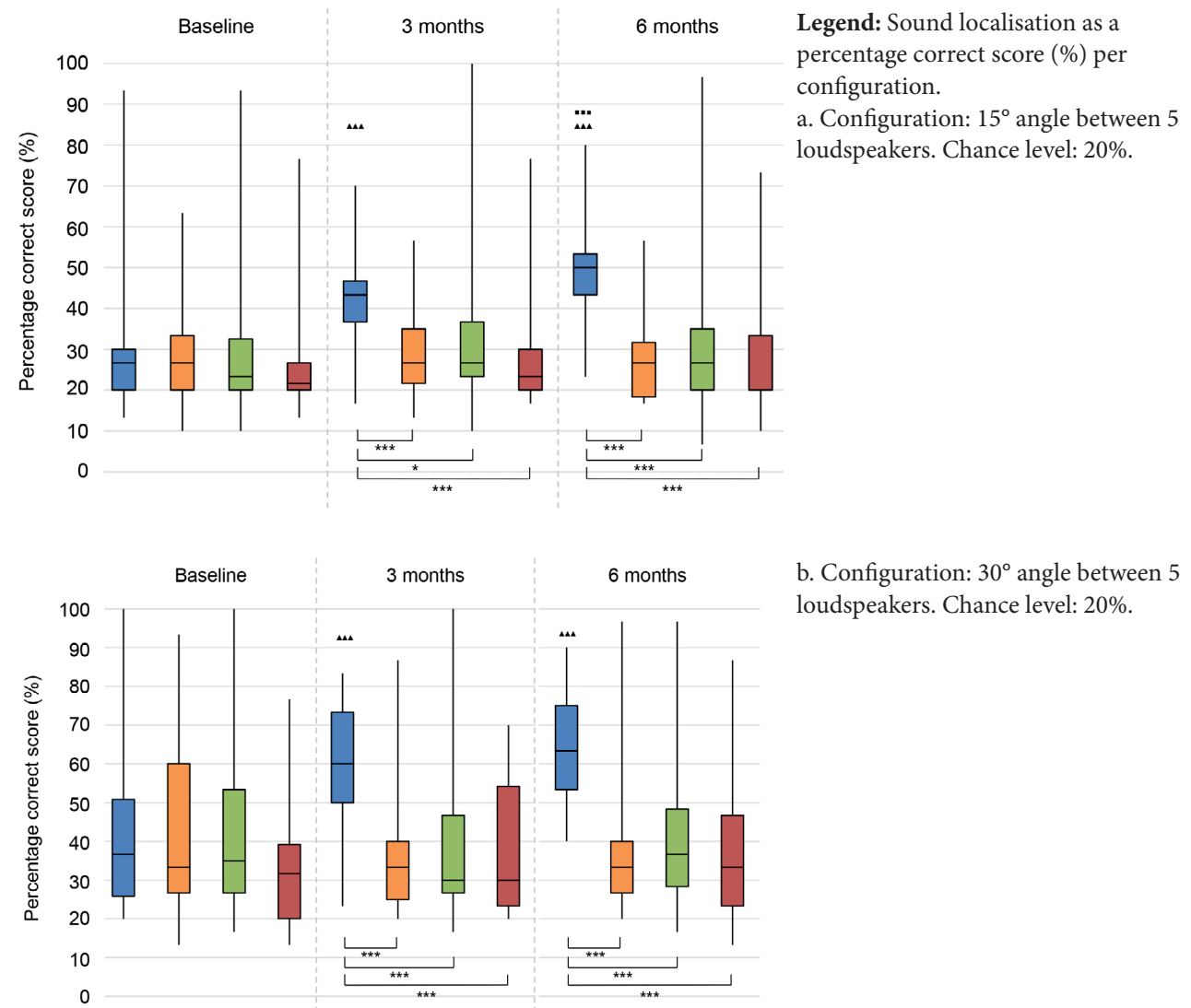

b. Configuration: $30^{\circ}$ angle between 5 loudspeakers. Chance level: $20 \%$.

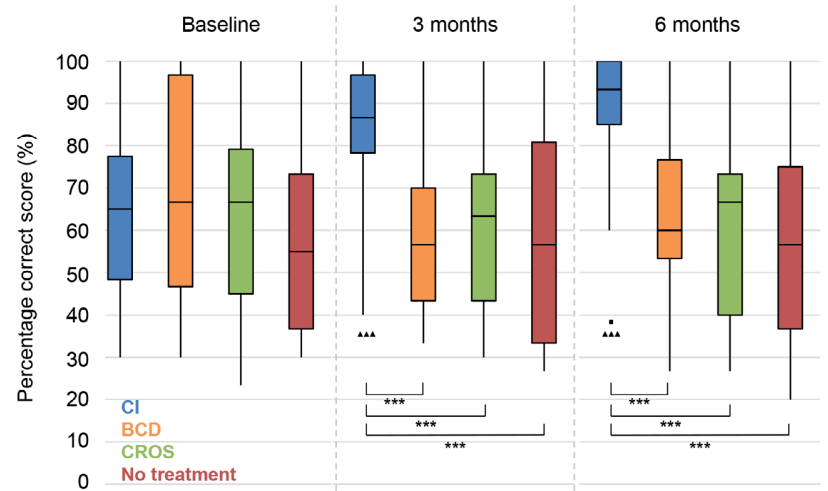

c. Configuration: $60^{\circ}$ angle between 3 loudspeakers. Chance level: $33.3 \%$. 


\section{Sound localisation}

See Figure 3 for the results on sound localisation. See Supplementary Material 3 for exact data. In all configurations and in all groups, a wide range in performance was observed.

The sound localisation of the CI group significantly improved between baseline and 3 and 6 months follow-up in all configurations. For the $15^{\circ}$ and $60^{\circ}$ configurations, there was also a statistically significant improvement between 3 and 6 months follow-up. There was no difference compared to baseline for the other groups.

At both 3 and 6 months follow-up, the CI group performed significantly better than all other groups. There were no statistically significant differences between the other groups. 


\section{Tinnitus}

At all follow-up moments and in all groups, there were some patients who indicated that they did not suffer from tinnitus (anymore) (Table 2). Since these patients had no tinnitus, they did not complete the TQ and THI at that moment. Patients with no tinnitus at baseline did not develop tinnitus after any intervention. See Supplementary Material 3 for exact data on the TQ and THI.

Table 2. Number of patients with and without tinnitus per group per follow-up moment

\begin{tabular}{llllllllllllllll}
\hline & CI & \multicolumn{4}{c}{ BCD } & \multicolumn{4}{c}{ CROS } & \multicolumn{3}{c}{ No treatment } \\
\hline Tinnitus? & Baseline & $\begin{array}{c}3 \\
\text { months }\end{array}$ & $\begin{array}{c}\mathbf{6} \\
\text { months }\end{array}$ & Baseline & $\begin{array}{c}3 \\
\text { months }\end{array}$ & $\begin{array}{c}\mathbf{6} \\
\text { months }\end{array}$ & Baseline & $\begin{array}{c}3 \\
\text { months }\end{array}$ & $\begin{array}{c}\mathbf{6} \\
\text { months }\end{array}$ & Baseline & $\begin{array}{c}3 \\
\text { months }\end{array}$ & $\begin{array}{c}\mathbf{6} \\
\text { months }\end{array}$ \\
\hline Yes & 26 & 20 & 21 & 22 & 18 & 21 & 33 & 31 & 28 & 24 & 21 & 24 \\
No & 2 & 5 & 5 & 3 & 3 & 2 & 1 & 1 & 2 & 2 & 3 & 3 \\
Missings & 0 & 2 & 1 & 0 & 2 & 0 & 0 & 2 & 1 & 0 & 2 & 0 \\
\hline $\begin{array}{l}\text { Total } \\
\text { number }\end{array}$ & 28 & 27 & 27 & 25 & 23 & 23 & 34 & 34 & 31 & 26 & 26 & 27 \\
\hline
\end{tabular}

\section{Legend}

In the row 'total number', the total number of patients per group and per follow-up moment is mentioned, which corresponds to the numbers shown in the Flow Diagram, Figure 1.

In the CI group, two patients did not suffer from tinnitus at baseline and they remained tinnitus-free after 3 and 6 months follow-up. Three patients reported to have no tinnitus anymore after cochlear implantation at 3 and 6 months follow-up.

In the BCD group, there were three patients who did not suffer from tinnitus at baseline. One of them was a patient who switched to the No treatment group after 3 and 6 months follow-up. The two other patients also did not suffer from tinnitus at 3 and 6 months follow-up. One other patient who suffered from tinnitus at baseline reported not to have tinnitus at 3 months follow-up, but the tinnitus had returned at 6 months follow-up.

In the CROS group, one patient had no tinnitus at baseline, 3 and 6 months follow-up. One other patient reported to have no tinnitus anymore at 6 months follow-up, whereas tinnitus was present at baseline and 3 months follow-up.

In the No treatment group, two patients were tinnitus-free at baseline, 3 and 6 months follow-up. The aforementioned patient with no tinnitus who switched from the BCD group was analysed in the No treatment group at 3 and 6 months follow-up.

Abbreviations:

$\mathrm{CI}=$ Cochlear Implant, $\mathrm{BCD}=$ Bone Conduction Device, $\mathrm{CROS}=$ Contralateral Routing of Sound hearing aid, $\mathrm{SD}$ $=$ standard deviation. 


\section{$T Q$}

There was a significant decrease in tinnitus-related distress for the CI group and the BCD group at 3 and 6 months follow-up compared to baseline (Figure 4a).

At 3 and 6 months follow-up, the CI group had significantly lower TQ scores than the No treatment group.

Figure 4. Questionnaires on tinnitus burden

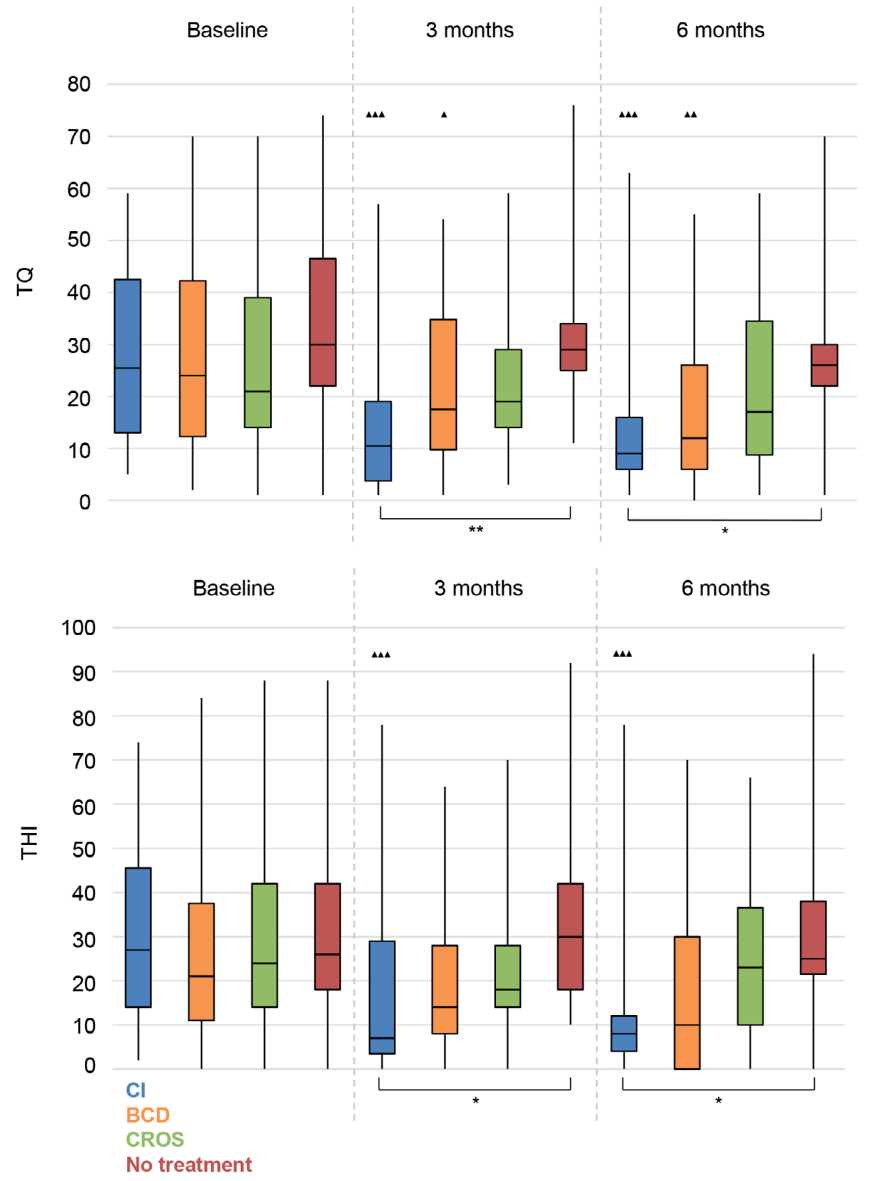

Legend:

a. Tinnitus Questionnaire (TQ)

b. Tinnitus Handicap Inventory (THI) 
THI

We measured a significant decrease in impact of tinnitus on daily life for the CI group at 3 and 6 months follow-up compared to baseline (Figure $4 \mathbf{b}$ ).

Again, at 3 and 6 months follow-up, the CI group had significantly lower THI scores than the No treatment group.

\section{Disease-specific QoL}

See Supplementary Material 3 for exact data on the disease-specific QoL questionnaires.

SSQ

\section{Speech-hearing}

There was a significant improvement (higher score) for all treatment groups at 3 and 6 months follow-up compared to baseline (Figure 5a). There was no significant change compared to baseline for the No treatment group.

At 3 and 6 months follow-up, the CI group had significantly higher median values compared to all other groups, and both the BCD and CROS groups scored better than the No treatment group. 
Figure 5. Speech, Spatial and Qualities of hearing questionnaire (SSQ)

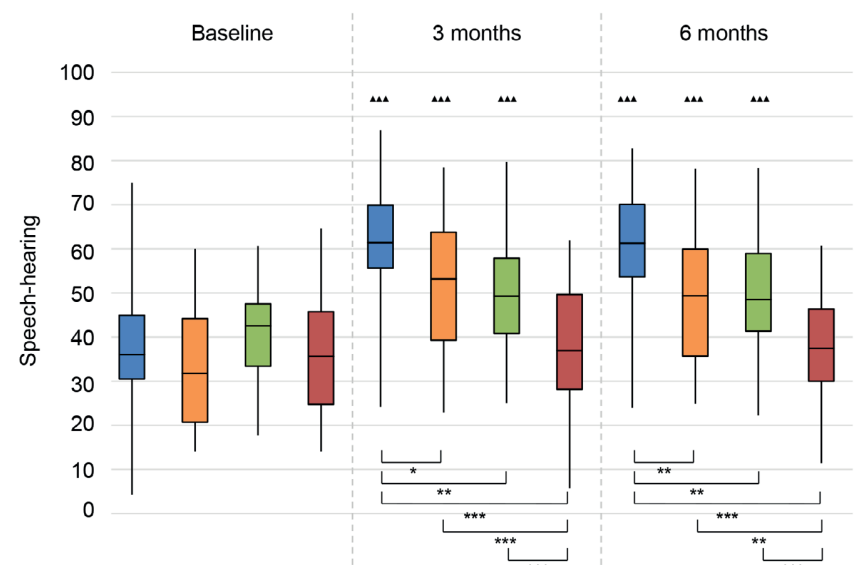

Legend:

a. Speech-hearing subscale

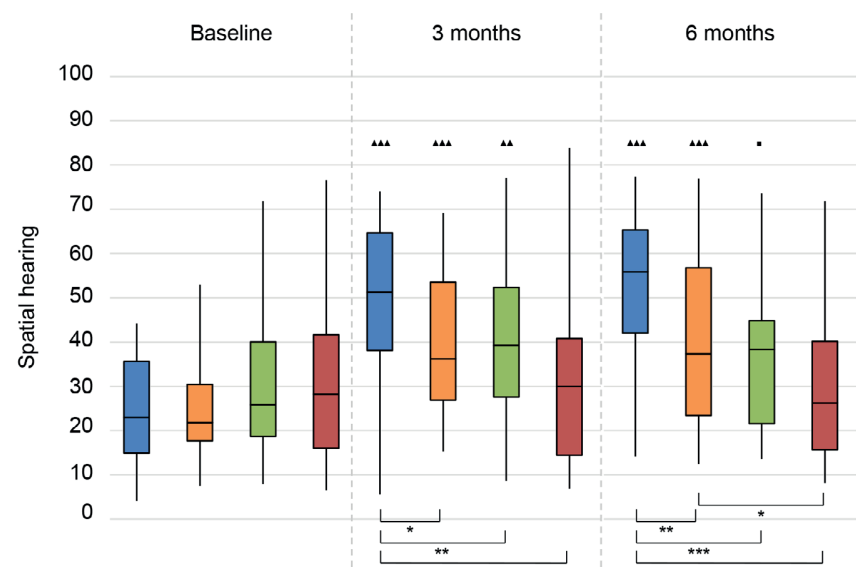

b. Spatial hearing subscale

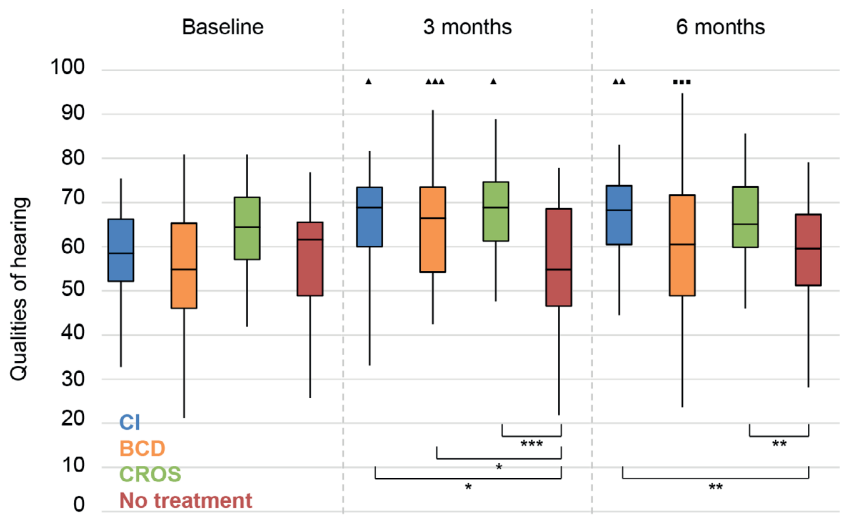




\section{Spatial hearing}

We observed a significant improvement for all treatment groups at 3 months follow-up, and for $\mathrm{CI}$ and BCD groups also at 6 months follow-up (Figure 5b). The score for the CROS group slightly decreased at 6 months follow-up and was therefore not significantly improved compared to baseline. There was no significant change for the No treatment group.

At 3 and 6 months follow-up, the scores for the CI group were significantly higher compared to all other groups. Also, at 6 months follow-up, the BCD group reached higher scores than the No treatment group.

\section{Qualities of hearing}

For all treatment groups, the scores at 3 months follow-up were significantly better than the baseline scores (Figure 5c). At 6 months follow-up, this improvement remained for only the CI group. For the BCD group, there was a significant deterioration between 3 and 6 months follow-up. There was no significant change for the No treatment group.

At 3 months follow-up, the scores of all treatment groups were significantly better than the scores of the No treatment group. At 6 months follow-up, the scores of only the CI and CROS groups were significantly better than the scores of the No treatment group.

\section{$A P H A B$}

\section{$\underline{\text { Ease of Communication (EC) }}$}

At 3 and 6 months follow-up, all treatment groups had an improvement (i.e. a decrease in APHAB score) on this subscale (Figure 6a).

At 3 and 6 months, all treatment groups had better scores than the No treatment group. The CI group had significantly better scores than the CROS group.

\section{Background noise (BN)}

All treatment groups had significantly improved scores at 3 and 6 months follow-up (Figure 6b). 
All treatment groups scored significantly better than the No treatment group at 3 and 6 months follow-up. The CI group had significantly better scores than the CROS group at 3 and 6 months follow-up, and also significantly better scores than the BCD group at 6 months follow-up.

\section{$\underline{\text { Reverberant conditions (RV) }}$}

The scores of all treatment groups had significantly improved at 3 and 6 months follow-up (Figure 6c). However, the scores for the CROS group significantly deteriorated between 3 and 6 months follow-up.

At 3 months follow-up, the CI group and the CROS group scored significantly better than the No treatment group. The CROS group also scored better than the BCD group. At 6 months follow-up, the CI group outperformed all other groups. All treatment groups scored better than the No treatment group.

\section{Aversiveness of sounds (AS)}

At 3 months follow-up, the scores of all treatment groups were better than the score of the No treatment group (Figure 6d). This benefit remained for the BCD and CI groups, whereas this benefit decreased for the CROS group, leading to a non-significant difference between baseline and 6 months follow-up for the CROS group.

At 3 months of follow-up, all treatment groups scored better than the No treatment group. At 6 months follow-up, the CI group scored significantly better than the CROS and No treatment groups. The scores of the BCD group were significantly better than the scores of the No treatment group. 


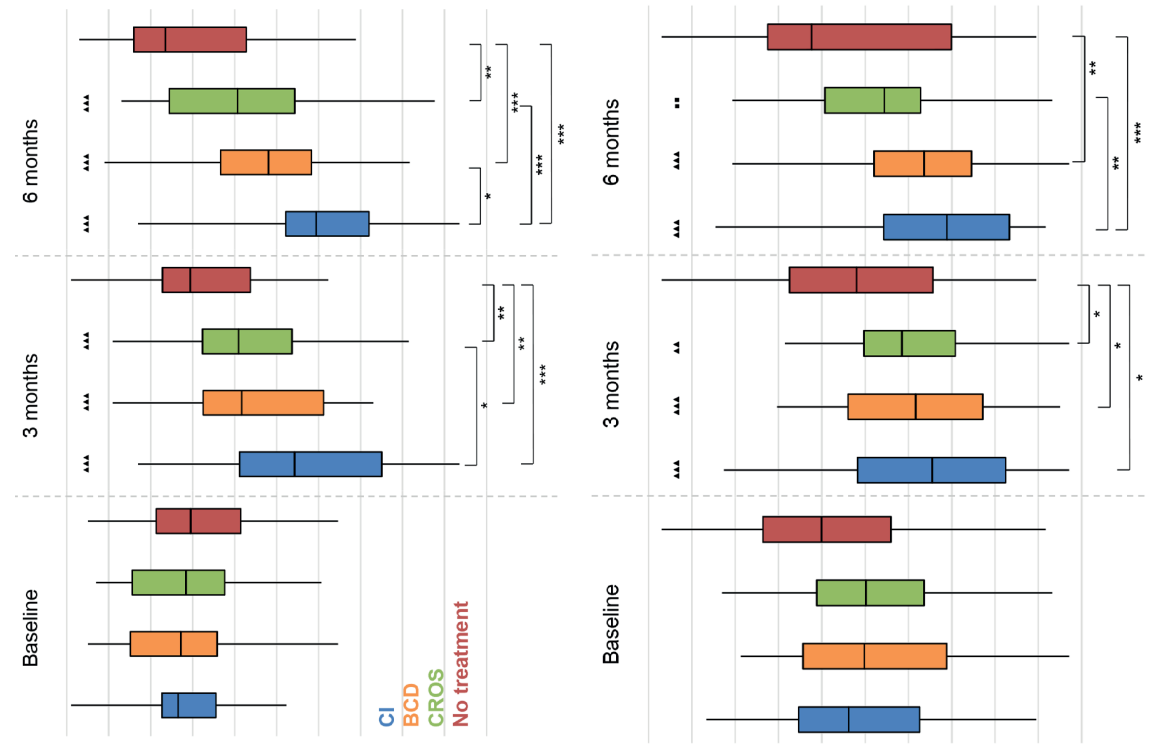

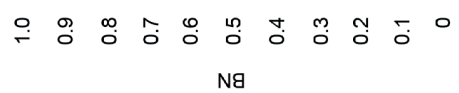

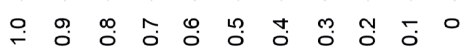

S $\forall$

包
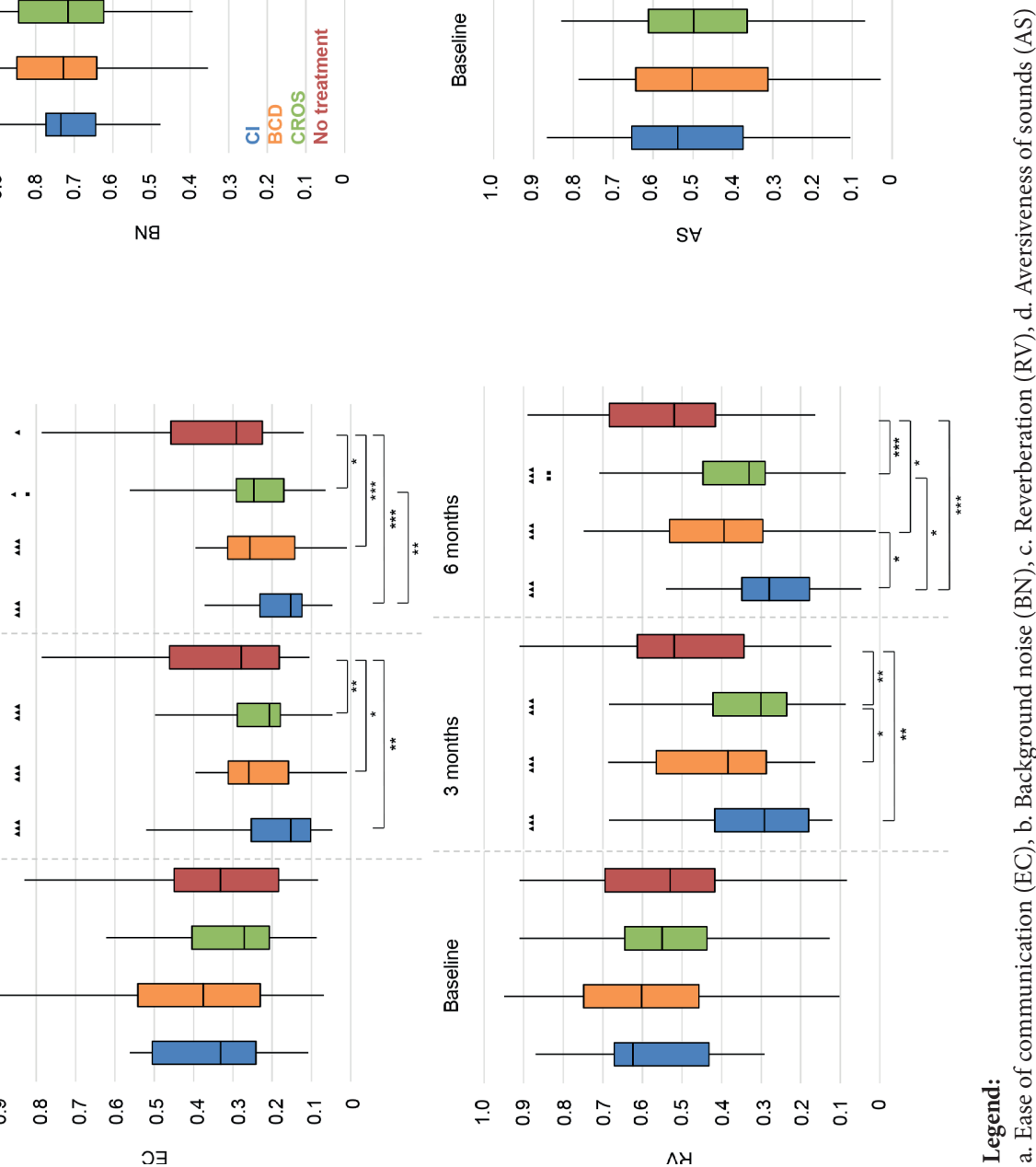


\section{GBI}

On the general subscale, the scores improved (i.e. $>0$ ) in all treatment groups. The CI group had a significantly better score than the CROS group at 3 months follow-up. Since the social support subscale and the physical health subscale of the GBI are calculated by adding the responses of three questions each, there was hardly any variability in the data (quartile 1, the median, and quartile 3 had exactly the same values; see Supplementary Material 3).

\section{Serious Adverse Events (SAEs)}

There were two related SAEs in the BCD group: two implant extrusions occurred; one patient was re-implanted, in the other case no re-implantation was performed at the patient's request.

There were also unrelated SAEs: in the CI group one patient suffered from a transient

ischemic attack several months after implantation; in the CROS group, one patient had a myocardial infarction for which he underwent percutaneous coronary intervention, and one patient had nasal polyps for which he underwent sinus surgery; in the No treatment group one patient had an arm fracture requiring surgery.

\section{DISCUSSION}

We presented the first results of this RCT with 120 included SSD patients evaluating CI, BCD, CROS, and No treatment at 3 and 6 months follow-up. The randomised allocation ensured equal distribution of known and unknown confounders amongst groups.

\section{Speech perception in noise}

On our primary outcome $\left(\mathrm{S}_{0} \mathrm{~N}_{0}\right)$, the CI group demonstrated a significant improvement compared to baseline, which can be attributed to the summation effect. Other studies did not measure an advantage in this configuration after 6 months CI use in SSD patients. ${ }^{12,30}$ In the $\mathrm{S}_{\mathrm{pe}} \mathrm{N}_{\mathrm{be}}$ configuration, all treatment options led to an improved speech perception in noise, since the sound from the side of the poor ear is delivered to the better ear. However, in the 
$\mathrm{S}_{\mathrm{be}} \mathrm{N}_{\mathrm{pe}}$ configuration the opposite happened: the BCD and CROS negated the positive effect of the head shadow as the noise on the poor side was sent to the better ear and thus impeded speech intelligibility. In this $\mathrm{S}_{\mathrm{be}} \mathrm{N}_{\mathrm{pe}}$ configuration, the BCD and CROS patients performed worse than at baseline, and worse than patients in the No treatment group at 3 and 6 months follow-up. In contrast to the BCD and CROS groups, there was no disadvantage for the CI patients, proving that the squelch effect is present, as was objectified before after cochlear implantation in patients with asymmetrical hearing loss. ${ }^{10,31}$

The speech perception in noise may have been influenced by the hearing thresholds in the better ear. However, there were no significant differences in $\mathrm{PTA}_{\mathrm{be}}$ between groups after treatment allocation (see Supplementary Material 2).

\section{Sound localisation}

Cochlear implantation led to a significant improvement in sound localisation in multiple configurations, which is in line with literature. ${ }^{10,12,15,18,19}$ The benefit of binaural input is present very early after CI activation, as was described by others. ${ }^{32}$ We found that the sound localisation ability of the CI patients improved from 3 to 6 months follow-up for two configurations $\left(15^{\circ}\right.$ and $\left.60^{\circ}\right)$, which is also in line with what other researchers have described. ${ }^{11,12,33}$ Nevertheless, sound localisation is still harder for SSD patients with a CI than for normal hearing subjects. ${ }^{22,34}$

In line with previous articles, we did not find improvement of sound localisation ability with BCD or CROS. ${ }^{9,35,36}$

\section{Tinnitus}

For the first time in 2008, Van de Heyning et al. reported a significant decrease in tinnitus distress (TQ) and tinnitus loudness (Visual Analogue Scale (VAS) score) following cochlear implantation in unilaterally deaf patients suffering from incapacitating tinnitus. ${ }^{5}$ Later, others reported beneficial results of electrical stimulation by cochlear implantation on tinnitus as 
well. ${ }^{15,16,18,30,37}$ An important issue to be mentioned is the absence of a minimum level of tinnitus distress to be eligible for inclusion in our study. Other study groups often required a minimum level of tinnitus distress, such as a minimum VAS score ${ }^{5,10,31}$ on tinnitus loudness of 6 out of 10 or a minimum THI score ${ }^{38,39}$ of 58 . This requirement explains their higher baseline values for tinnitus distress. Despite our lower baseline values, we also objectified a significant decrease in tinnitus distress after 3 and 6 months for the CI patients.

Interestingly, we identified a significant reduction on tinnitus distress after BCD implantation with the TQ, without a significant change in tinnitus-related effects on daily life as measured by the THI. One other study found a reduction of the impact of tinnitus on daily life after $\mathrm{BCD} .{ }^{40}$ The mechanism is unclear, but stimulation of the contralateral auditory pathway may play an important role in the experienced tinnitus suppression. ${ }^{40}$

Some CI patients reported reduction of tinnitus as the most important advantage of their CI, rather than improved speech perception in noise or sound localisation. This could be an important advantage that should be mentioned when counselling SSD patients on expected outcomes of CI.

\section{Disease-specific QoL}

For most subscales on the disease-specific QoL questionnaires, there was an improvement for all treatment groups compared to baseline, and compared to the No treatment group at 3 and 6 months follow-up. Our SSQ results are in line with the results of studies that also used the SSQ questionnaire in SSD patients after cochlear implantation. ${ }^{30,37}$

Although the APHAB questionnaire has not been widely used in reports on cochlear implantation for SSD, we implemented this questionnaire because it has been extensively used to evaluate BCD and CROS. ${ }^{9}$ Also on the APHAB we found an improvement for all treatment groups on most subscales, which reflects findings by others as well. ${ }^{9,41}$ 


\section{Trial period}

The proportion of patients opting for a BCD after the trial period in our study (29.4\%) was on the low end of the range described in literature for patients opting for a $\mathrm{BCD}$ after a $\mathrm{BCD}$ trial period $(32.0-69.6 \%) .{ }^{42}$ Patients in our study were offered a cross-over design which enabled them to test two devices, which may explain the lower proportion (noticeably, $40.0 \%$ of patients opted for CROS after the trial period). Moreover, the proportion of patients opting for a $\mathrm{BCD}$ could have been influenced by the type of $\mathrm{BCD}$ offered during the trial period; we noticed that the satisfaction with the newer Baha 5 Power was significantly higher than with the older Baha BP110 (45.5\% versus 19.2\% opted for BCD after the trial period, respectively).

Other studies have also provided a trial with BCD or CROS to SSD patients. Though, due to lack of reported outcomes in these studies, comparison with our results is hindered. For example, Arndt et al. provided a CI to 11 SSD patients that were not satisfied with the effects of BCD or CROS in a trial period..$^{30}$ However, the proportion of patients that opted for $\mathrm{BCD}$ or CROS after the trial period was not mentioned, and no objective outcomes of hearing performance were published from these satisfied patients. Likewise, SSD patients were offered a CI in a Swiss study if they had unsatisfactory benefit from a trial with CROS or BCD: forty-four of 54 study patients did not proceed to cochlear implantation. ${ }^{43}$ Unfortunately, there were no results available from those patients who opted for BCD or CROS. Of the 10 patients that were implanted, only results with CI were published and not from the trial period with CROS or BCD. ${ }^{43}$ Kurz et al. implemented the study design that was proposed at an international consensus meeting ${ }^{1}$, and found that 16 of 34 patients decided not to opt for cochlear implantation after a trial period with BCD and CROS (3 weeks each). ${ }^{12}$

As an advantage over studies with a trial period of BCD and CROS before the option to choose for $\mathrm{CI}$, we were able to make a longitudinal between-group comparison comparing $\mathrm{CI}, \mathrm{BCD}, \mathrm{CROS}$, and no treatment. With our study design we diminished the influence of the opinion of patients on CI outcome by previous BCD or CROS treatments, and also diminished the influence on patients' opinions on BCD and CROS. With a more extended 
analysis of the trial period, we hope to identify prognostic factors that may predict if BCD or CROS are successful in specific categories of patients.

\section{Strengths}

There are several strengths that characterize our trial. Firstly, we randomly allocated patients to intervention groups. Our random allocation ensured equal distribution of patient characteristics across groups, thereby eliminating the influence of differences in these characteristics on the outcomes. ${ }^{44,45}$ Secondly, we compared CI to the treatment options for patients with SSD currently available in many countries. Thirdly, our design provided not only a within-subject comparison, but also allowed a between-group comparison. It also enabled us to evaluate the performance following SSD in the No treatment group. Finally, we had very few missing data permitting a complete case-analysis.

\section{Limitations}

Our study also has limitations. First, following the stringent in- and exclusion criteria, we included only a subset of patients with SSD, which may limit the generalisability of our results. Moreover, our study patients were highly motivated to return for follow-up visits, whereas in real-life compliance may not be as high. Possibly, non-use of initially started treatment may occur when follow-up time is longer. ${ }^{17,33}$ We therefore implemented a follow-up period of five years. Second, neither patients nor personnel were blinded for the allocated intervention. Given the nature of the interventions, blinding was impossible to achieve, which may have influenced outcomes; this is a well-known problem in nonpharmacological studies. ${ }^{46}$ Third, due to the long inclusion period of our trial, we implemented newly developed devices in the trial. Consequently, not all patients tested the same BCD in the trial period, or had the same type of CROS or CI fitted. We feel this is part of conducting a multi-annual trial and reflects technological advancements similar to clinical care settings. Because of standardised fitting strategies for all devices, we think the groups are still comparable. Fourth, the TQ and THI 
are not designed to measure treatment effects. ${ }^{47}$ To measure treatment-related changes in tinnitus intrusiveness and severity, the more recently developed Tinnitus Functional Index ${ }^{48}$ is recommended nowadays. Nevertheless, the commonly used THI makes comparisons with other studies possible. ${ }^{16}$ Furthermore, both the TQ and the THI have high internal consistency, high convergence and discriminant validity and good change sensitivity. ${ }^{49}$ Thus, both questionnaires appear appropriate to evaluate the effects of tinnitus treatments. ${ }^{49}$ Finally, we only measured the median duration of CI use per day, and not the median duration of BCD and CROS use per day. Data of device use from the BCD and CROS devices would have given us insight in the satisfaction with the devices as mirrored from duration of use per day; unfortunately, this data was unavailable.

\section{Future perspectives}

In this paper we presented the first results of the CINGLE-trial. ${ }^{20}$ Long-term results will follow. Aside from the reported outcome measures, which show the individual benefits, analysis of the costs of the treatment options is necessary to reveal societal benefits. This information will allow health care committees to perform a cost utility analysis, which will help in the consideration whether or not to reimburse cochlear implantation for patients with SSD. Interestingly, a recent modelling study on patients with SSD found that cochlear implantation may be cost-effective compared with no intervention, but bone-conduction implants were unlikely to be cost-effective. ${ }^{50}$ Studies comparing CI to other treatment options for SSD and conducting a cost utility analysis are expected in the near future. ${ }^{51,52}$ Finally, others work on developing a minimum set of core outcomes for use in future trials of SSD interventions. ${ }^{53}$

\section{Conclusion}

In this RCT, we compared CI, BCD, CROS, and No treatment for patients with SSD. Speech perception in noise improved in all configurations for the CI group, whereas speech perception in noise improved or deteriorated for the BCD and CROS groups depending on 
the configuration. Sound localisation improved in the CI group only. Patients with no tinnitus at baseline did not develop tinnitus after any intervention. After cochlear implantation, three patients reported that their tinnitus had completely disappeared. On both the TQ and THI, a decrease in tinnitus burden was detected for the CI group. In general, all treatment options improved disease-specific QoL on most subscales of the used questionnaires.

Results from long-term follow-up moments will be presented in the future, as well as an analysis of the BCD/CROS trial period and a more detailed analysis of the tinnitus burden. Finally, cost utility analyses on treatment options for SSD are needed to evaluate if cochlear implantation should be reimbursed. 


\section{SUPPLEMENTARY MATERIAL 1:}

\section{Explanation of numbers per group at different follow-up moments}

\section{$\underline{\text { After randomisation }}$}

In the CI group ( $\mathrm{n}=29$ patients randomised), one patient decided not to proceed with cochlear implantation because he found cochlear implantation too invasive, and withdrew from the study. Therefore, 28 patients were analyzed at baseline. One patient was lost to follow-up before the 3 months follow-up moment, and thus 27 patients were analyzed at 3 and 6 months follow-up. During the first 6 months of follow-up, there were no non-users.

In the 'first $\mathrm{BCD}$, then CROS' group ( $\mathrm{n}=45$ patients randomised), four patients decided to terminate the study before the end of the trial period (three patients were disappointed that they were not randomised to the CI group, one patient had his CI reimbursed by exception for his work with 'special needs' (police officer)). After the trial period 10 patients opted for the BCD, 17 patients chose for CROS, and 14 patients preferred no treatment.

In the 'first CROS, then BCD' group ( $\mathrm{n}=46$ patients randomised), two patients decided to terminate the study before the end of the trial period (both lacked motivation to return for follow-up visits). After the trial period 15 patients opted for the $\mathrm{BCD}, 17$ patients chose for CROS and 12 patients preferred no treatment.

\section{$\underline{\text { After allocation of treatment }}$}

In the BCD group ( $\mathrm{n}=25$ patients after trial period), one patient decided not to undergo surgery and terminated study participation. One patient was implanted, but due to recurrent skin infections around the BCD screw, it had to be removed and was not re-inserted. This patient was further analyzed in the No treatment group at 3 and 6 months follow-up. Hence, 25 patients were analyzed at baseline, and 23 patients were analyzed in the BCD group at 3 and 6 months follow-up.

In the CROS group ( $n=34$ patients after trial period), two patients did not use their 
CROS anymore after the 3 months follow-up moment, and were therefore measured without their device and analyzed in the No treatment group at 6 months. One patient was lost to follow-up after 3 months follow-up. Hence, 34 patients were analyzed in the CROS group at baseline and 3 months, and 31 patients were analyzed at 6 months follow-up.

In the No treatment group ( $\mathrm{n}=26$ patients after trial period), one patient ended study participation before the 3 months follow-up moment because the follow-up visits were too demanding. As mentioned, one patient from the BCD group was analyzed in the No treatment group at 3 and 6 months. Another patient was lost to follow-up after 3 months. As mentioned, two patients from the CROS group were analyzed in the No treatment group at 6 months. So in total, 26 patients were analyzed in the No treatment group at baseline and 3 months, and 27 patients were analyzed at 6 months follow-up. 


\section{SUPPLEMENTARY MATERIAL 2:}

Patient characteristics after allocation of treatment (i.e. CI activation, BCD implantation and CROS fitting).

\begin{tabular}{|c|c|c|c|c|c|}
\hline & CI & BCD & CROS & No Treatment & Statistics \\
\hline \multicolumn{6}{|l|}{ Gender } \\
\hline Male:Female & $13: 14$ & $8: 15$ & $21: 13$ & $10: 16$ & $\mathrm{~ns}^{\mathrm{a}}$ \\
\hline \multicolumn{6}{|l|}{ Age at inclusion (years) } \\
\hline Mean (SD) & $52.5(13.1)$ & $55.6(8.4)$ & $52.1(12.0)$ & $52.1(13.3)$ & $\mathrm{ns}^{\mathrm{b}}$ \\
\hline \multicolumn{6}{|l|}{ PTA $_{\text {be }}(0.5-4 \mathrm{kHz})(\mathrm{dB})$} \\
\hline Mean (SD) & $15.0(6.9)$ & $14.2(6.5)$ & $16.0(6.6)$ & $15.2(7.3)$ & \multirow{2}{*}{$\mathrm{ns}^{\mathrm{c}}$} \\
\hline Median [range] & $15.0[5.0-30.0]$ & $12.5[3.8-28.8]$ & $16.3[5.0-27.5]$ & $17.5[2.5-30.0]$ & \\
\hline \multicolumn{6}{|l|}{ Duration of deafness (years) } \\
\hline Mean (SD) & $3.0(2.9)$ & $3.0(3.1)$ & $2.7(3.0)$ & $3.1(2.9)$ & \multirow{2}{*}{$\mathrm{ns}^{\mathrm{c}}$} \\
\hline Median [range] & $1.8[0.3-10.0]$ & $2.2[0.3-10.0]$ & $1.3[0.3-10.0]$ & $2.2[0.3-10.0]$ & \\
\hline \multicolumn{6}{|l|}{ Etiology } \\
\hline Unknown & 5 & 3 & 5 & 13 & \multirow{7}{*}{$\mathrm{ns}^{\mathrm{a}}$} \\
\hline Iatrogenic & 1 & 0 & 0 & 1 & \\
\hline Sudden deafness & 14 & 15 & 18 & 8 & \\
\hline Labyrinthitis & 4 & 2 & 5 & 1 & \\
\hline Infection (not otherwise specified) & 0 & 0 & 2 & 1 & \\
\hline Ménière’s disease & 3 & 2 & 3 & 0 & \\
\hline Traumatic & 0 & 1 & 1 & 2 & \\
\hline
\end{tabular}

Legend: There were no statistically significant differences between the groups.

Abbreviations:

$\mathrm{CI}=$ Cochlear Implant, BCD = Bone Conduction Device, CROS $=$ Contralateral Routing of Sound hearing aid, $\mathrm{ns}=$ not significant $(p>0.05), \mathrm{SD}=$ standard deviation, $\mathrm{PTA}_{\mathrm{be}}=$ Pure Tone Average threshold of the best ear at $0.5,1,2,4 \mathrm{kHz}(\mathrm{dB})$.

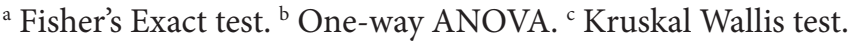




\section{SUPPLEMENTARY MATERIAL 3: data tables}

Table 1: Speech perception in noise

\begin{tabular}{lccccccccccccc}
\hline \multicolumn{10}{c}{$\mathbf{S}_{0} \mathbf{N}_{0}$} \\
\hline \multicolumn{10}{c}{ Baseline } & \multicolumn{10}{c}{ 3 months } \\
\hline & CI & BCD & CROS & No Tr & CI & BCD & CROS & No Tr & CI & BCD & CROS & No Tr \\
\hline min & -5.0 & -5.9 & -5.9 & -7.2 & -6.9 & -5.6 & -5.9 & -6.9 & -5.9 & -5.0 & -6.6 & -6.6 \\
iqr 1 & -4.1 & -3.4 & -4.3 & -4.4 & -5.0 & -3.8 & -3.8 & -4.4 & -5.5 & -4.2 & -3.9 & -4.1 \\
med & -2.8 & -2.2 & -3.6 & -3.0 & -4.4 & -3.1 & -3.4 & -2.5 & -4.1 & -3.4 & -3.4 & -3.4 \\
iqr 3 & -1.8 & -0.9 & -2.5 & -1.0 & -3.0 & -1.6 & -3.1 & -1.8 & -2.8 & -1.7 & -2.5 & -1.9 \\
max & -0.6 & 12.8 & -0.3 & 3.1 & -0.9 & 0.9 & -0.6 & 1.3 & 0.0 & 10.6 & 0.3 & 2.5 \\
\hline $\mathrm{n}$ & 28 & 25 & 34 & 26 & 27 & 23 & 33 & 24 & 27 & 23 & 31 & 27 \\
\hline
\end{tabular}

\begin{tabular}{|c|c|c|c|c|c|c|c|c|c|c|c|c|}
\hline & \multicolumn{12}{|c|}{$\mathrm{S}_{\mathrm{pe}} \mathrm{N}_{\mathrm{be}}$} \\
\hline & \multicolumn{4}{|c|}{ Baseline } & \multicolumn{4}{|c|}{3 months } & \multicolumn{4}{|c|}{6 months } \\
\hline & CI & BCD & CROS & No $\operatorname{Tr}$ & CI & BCD & CROS & No $\operatorname{Tr}$ & CI & BCD & CROS & No $\operatorname{Tr}$ \\
\hline $\min$ & 2.2 & 0.0 & -0.9 & 0.3 & -6.6 & -5.0 & -6.6 & -0.6 & -6.3 & -6.3 & -7.8 & -0.3 \\
\hline iqr 1 & 3.4 & 3.8 & 2.9 & 3.8 & -3.1 & -2.2 & -2.8 & 4.0 & -3.8 & -1.4 & -2.3 & 3.6 \\
\hline med & 4.7 & 5.0 & 4.4 & 4.7 & -1.9 & -0.9 & -0.9 & 4.7 & -2.2 & -0.3 & -1.3 & 5.0 \\
\hline iqr 3 & 6.6 & 6.3 & 5.6 & 6.2 & -0.5 & 2.2 & 0.0 & 5.9 & -1.4 & 1.6 & 0.8 & 7.0 \\
\hline $\max$ & 7.8 & 19.4 & 10.6 & 10.6 & 2.2 & 5.6 & 4.7 & 10.3 & 1.9 & 3.8 & 2.2 & 11.6 \\
\hline $\mathrm{n}$ & 28 & 25 & 34 & 26 & 27 & 23 & 33 & 24 & 27 & 23 & 31 & 27 \\
\hline
\end{tabular}

\begin{tabular}{lrrrrrrrrrrrrr}
\hline \multicolumn{10}{c}{$\mathbf{S}_{\mathrm{be}} \mathbf{N}_{\mathrm{pe}}$} \\
\hline \multicolumn{10}{c}{ Baseline } & \multicolumn{10}{c}{ 3 months } \\
\hline & CI & BCD & CROS & No Tr & CI & BCD & CROS & No Tr & CI & BCD & CROS & No Tr \\
\hline min & -14.1 & -14.7 & -15.6 & -15.0 & -15.3 & -12.5 & -11.9 & -14.4 & -16.3 & -11.9 & -12.5 & -14.1 \\
iqr 1 & -11.9 & -11.6 & -12.2 & -11.8 & -13.1 & -8.1 & -8.4 & -12.5 & -12.8 & -9.1 & -8.4 & -12.8 \\
med & -10.9 & -10.0 & -10.9 & -9.7 & -11.9 & -7.2 & -6.9 & -10.8 & -11.6 & -7.8 & -6.3 & -10.6 \\
iqr 3 & -9.6 & -8.4 & -8.8 & -7.9 & -10.3 & -5.9 & -5.0 & -9.1 & -10.3 & -5.8 & -5.0 & -8.6 \\
max & -6.3 & 11.6 & -5.0 & -5.0 & -5.9 & 4.4 & 2.2 & -5.0 & -6.6 & 10.3 & 0.9 & -2.8 \\
\hline $\mathrm{n}$ & 28 & 25 & 34 & 26 & 27 & 23 & 33 & 24 & 27 & 23 & 31 & 27 \\
\hline
\end{tabular}


Legend: Speech reception threshold in noise (SRTn) (in $\mathrm{dB}$ ) at which patients were able to repeat $50 \%$ of the sentences correctly.

$\mathrm{CI}=$ cochlear implant, $\mathrm{BCD}=$ Bone Conduction Device, CROS = Contralateral Routing of Sound hearing aid, No $\operatorname{Tr}=$ No treatment

$\mathrm{S}_{0} \mathrm{~N}_{0}$ : speech and noise from the front, $\mathrm{S}_{\mathrm{pe}} \mathrm{N}_{\mathrm{be}}$ = speech presented to the poor ear, noise presented to the better ear, $\mathrm{S}_{\mathrm{be}} \mathrm{N}_{\mathrm{pe}}=$ speech presented to the better ear, noise presented to the poor ear.

Minimum (min) and maximum $(\max )$ values are presented, as well as first and third quartiles (iqr 1 and iqr 3, respectively), medians (med) and number of measurements (n). 
Table 2: Sound localisation

\begin{tabular}{lcrrrrrrrrrrr}
\hline \multicolumn{10}{c}{ Baseline } & \multicolumn{10}{c}{$\mathbf{1 5}^{\circ}$} \\
\hline \multicolumn{10}{c}{ CI } & BCD & \multicolumn{10}{c}{ CROS } & No Tr & CI & BCD & CROS & No Tr & CI & BCD & CROS & No Tr \\
\hline min & 13.3 & 10.0 & 10.0 & 13.3 & 16.7 & 13.3 & 10.0 & 16.7 & 23.3 & 16.7 & 6.7 & 10.0 \\
iqr 1 & 20.0 & 20.0 & 20.0 & 20.0 & 36.7 & 21.7 & 23.3 & 20.0 & 43.3 & 18.3 & 20.0 & 20.0 \\
med & 26.7 & 26.7 & 23.3 & 21.7 & 43.3 & 26.7 & 26.7 & 23.3 & 50.0 & 26.7 & 26.7 & 20.0 \\
iqr 3 & 30.0 & 33.3 & 32.5 & 26.7 & 46.7 & 35.0 & 36.7 & 30.0 & 53.3 & 31.7 & 35.0 & 33.3 \\
max & 93.3 & 63.3 & 93.3 & 76.7 & 70.0 & 56.7 & 100.0 & 76.7 & 80.0 & 56.7 & 96.7 & 73.3 \\
\hline n & 28 & 25 & 34 & 26 & 27 & 23 & 33 & 24 & 27 & 23 & 31 & 27 \\
\hline
\end{tabular}

\begin{tabular}{|c|c|c|c|c|c|c|c|c|c|c|c|c|}
\hline & \multicolumn{12}{|c|}{$30^{\circ}$} \\
\hline & \multicolumn{4}{|c|}{ Baseline } & \multicolumn{4}{|c|}{3 months } & \multicolumn{4}{|c|}{6 months } \\
\hline & CI & BCD & CROS & No $\mathrm{Tr}$ & CI & BCD & CROS & No $\mathrm{Tr}$ & CI & BCD & CROS & No $\mathrm{Tr}$ \\
\hline $\min$ & 20.0 & 13.3 & 16.7 & 13.3 & 23.3 & 20.0 & 16.7 & 20.0 & 40.0 & 20.0 & 16.7 & 13.3 \\
\hline iqr 1 & 25.8 & 26.7 & 26.7 & 20.0 & 50.0 & 25.0 & 26.7 & 23.3 & 53.3 & 26.7 & 28.3 & 23.3 \\
\hline med & 36.7 & 33.3 & 35.0 & 31.7 & 60.0 & 33.3 & 30.0 & 30.0 & 63.3 & 33.3 & 36.7 & 33.3 \\
\hline iqr 3 & 50.8 & 60.0 & 53.3 & 39.2 & 73.3 & 40.0 & 46.7 & 54.2 & 75.0 & 40.0 & 48.3 & 46.7 \\
\hline $\max$ & 100.0 & 93.3 & 100.0 & 76.7 & 83.3 & 86.7 & 100.0 & 70.0 & 90.0 & 96.7 & 96.7 & 86.7 \\
\hline $\mathrm{n}$ & 28 & 25 & 34 & 26 & 27 & 23 & 33 & 24 & 27 & 23 & 31 & 27 \\
\hline
\end{tabular}

\begin{tabular}{lrrrrrrrrrrrr}
\hline \multicolumn{10}{c}{$\mathbf{6 0}^{\circ}$} \\
\hline \multicolumn{10}{c}{ Baseline } & \multicolumn{10}{c}{ 3 months } \\
\hline & CI & BCD & CROS & No Tr & CI & BCD & CROS & No Tr & CI & BCD & CROS & No Tr \\
\hline min & 30.0 & 30.0 & 23.3 & 30.0 & 40.0 & 33.3 & 30.0 & 26.7 & 60.0 & 26.7 & 26.7 & 20.0 \\
iqr 1 & 48.3 & 46.7 & 45.0 & 36.7 & 78.3 & 43.3 & 43.3 & 33.3 & 85.0 & 53.3 & 40.0 & 36.7 \\
med & 65.0 & 66.7 & 66.7 & 55.0 & 86.7 & 56.7 & 63.3 & 56.7 & 93.3 & 60.0 & 66.7 & 56.7 \\
iqr 3 & 77.5 & 96.7 & 79.2 & 73.3 & 96.7 & 70.0 & 73.3 & 80.8 & 100.0 & 76.7 & 73.3 & 75.0 \\
max & 100.0 & 100.0 & 100.0 & 100.0 & 100.0 & 100.0 & 100.0 & 100.0 & 100.0 & 100.0 & 100.0 & 100.0 \\
\hline $\mathrm{n}$ & 28 & 25 & 34 & 26 & 27 & 23 & 33 & 24 & 27 & 23 & 31 & 27 \\
\hline
\end{tabular}

\section{Legend:}

Values in percentage correct score (\%).

$\mathrm{CI}=$ cochlear implant, $\mathrm{BCD}=$ Bone Conduction Device, $\mathrm{CROS}=$ Contralateral Routing of Sound hearing aid, No $\operatorname{Tr}=$ No treatment

Configurations:

15 5 loudspeakers separated by an angle of $15^{\circ}\left(-30^{\circ},-15^{\circ}, 0^{\circ},+15^{\circ}\right.$, and $\left.+30^{\circ}\right)$.

$30^{\circ}$ : 5 loudspeakers separated by an angle of $30^{\circ}\left(-60^{\circ},-30^{\circ}, 0^{\circ},+30^{\circ}\right.$, and $\left.+60^{\circ}\right)$.

$60^{\circ}: 3$ loudspeakers separated by an angle of $60^{\circ}\left(-60^{\circ}, 0^{\circ}\right.$, and $\left.+60^{\circ}\right)$.

Minimum ( $\min )$ and maximum $(\max )$ values are presented, as well as first and third quartiles (iqr 1 and iqr 3, respectively), medians (med) and number of measurements (n). 
Table 3: Questionnaires on tinnitus burden

Table 3a: TQ

\begin{tabular}{rrrrrrrrrrrrrr}
\hline \multicolumn{10}{c}{ TQ } \\
\hline \multicolumn{10}{c}{ Baseline } & \multicolumn{10}{c}{ 3 months } \\
\hline & CI & BCD & CROS & No Tr & CI & BCD & CROS & No Tr & CI & BCD & CROS & No Tr \\
\hline min & 5.0 & 2.0 & 1.0 & 1.0 & 1.0 & 1.0 & 3.0 & 11.0 & 1.0 & 0.0 & 1.0 & 1.0 \\
iqr 1 & 13.0 & 12.3 & 14.0 & 22.0 & 3.8 & 9.8 & 14.0 & 25.0 & 6.0 & 6.0 & 8.8 & 22.0 \\
med & 25.5 & 24.0 & 21.0 & 30.0 & 10.5 & 17.5 & 19.0 & 29.0 & 9.0 & 12.0 & 17.0 & 26.0 \\
iqr 3 & 42.5 & 42.3 & 39.0 & 46.5 & 19.0 & 34.8 & 29.0 & 34.0 & 16.0 & 26.0 & 34.5 & 30.0 \\
max & 59.0 & 70.0 & 70.0 & 74.0 & 57.0 & 54.0 & 59.0 & 76.0 & 63.0 & 55.0 & 59.0 & 70.0 \\
\hline $\mathrm{n}$ & 26 & 22 & 33 & 24 & 20 & 18 & 31 & 21 & 21 & 21 & 28 & 24 \\
\hline
\end{tabular}

Table 3b: THI

\begin{tabular}{lrrrrrrrrrrrrr}
\hline \multicolumn{10}{c}{ THI } \\
\hline \multicolumn{10}{c}{ Baseline } & \multicolumn{10}{c}{ 3 months } & \multicolumn{1}{c}{ 6 months } \\
\hline min & CI & BCD & CROS & No Tr & CI & BCD & CROS & No Tr & CI & BCD & CROS & No Tr \\
\hline iqr 1 & 2.0 & 0.0 & 0.0 & 0.0 & 0.0 & 0.0 & 0.0 & 10.0 & 0.0 & 0.0 & 0.0 & 0.0 \\
med & 14.0 & 11.0 & 14.0 & 18.0 & 3.5 & 8.0 & 14.0 & 18.0 & 4.0 & 0.0 & 10.0 & 21.5 \\
iqr 3 & 27.0 & 21.0 & 24.0 & 26.0 & 7.0 & 14.0 & 18.0 & 30.0 & 8.0 & 10.0 & 23.0 & 25.0 \\
max & 45.5 & 37.5 & 42.0 & 42.0 & 29.0 & 28.0 & 28.0 & 42.0 & 12.0 & 30.0 & 36.5 & 38.0 \\
\hline $\mathrm{n}$ & 74.0 & 84.0 & 88.0 & 88.0 & 78.0 & 64.0 & 70.0 & 92.0 & 78.0 & 70.0 & 66.0 & 94.0 \\
\hline & 26 & 22 & 33 & 24 & 20 & 18 & 31 & 21 & 21 & 21 & 28 & 24 \\
\hline
\end{tabular}

Legend:

Table 3a: results on the TQ. Possible range of score $0.0-80.0$.

Table 3b: results on the THI. Possible range of score $0.0-100.0$.

$\mathrm{CI}=$ cochlear implant, $\mathrm{BCD}=$ Bone Conduction Device, $\mathrm{CROS}=$ Contralateral Routing of Sound hearing aid, No $\mathrm{Tr}=$ No treatment

At baseline and at 3 and 6 months follow-up, patients indicated whether they had tinnitus or not. Patients with no tinnitus, did not complete the questionnaires on tinnitus burden.

Minimum ( $\min )$ and maximum $(\max )$ values are presented, as well as first and third quartiles (iqr 1 and iqr 3, respectively), medians (med) and number of measurements (n). 
Table 4: SSQ questionnaire

\begin{tabular}{rrrrrrrrrrrrrr}
\hline \multicolumn{10}{c}{ Speech-hearing } \\
\hline \multicolumn{10}{c}{ Baseline } & \multicolumn{10}{c}{ 3 months } \\
\hline & CI & BCD & CROS & No Tr & CI & BCD & CROS & No Tr & CI & BCD & CROS & No Tr \\
\hline min & 4.3 & 14.1 & 17.7 & 14.1 & 24.2 & 22.9 & 25.0 & 5.8 & 24.0 & 24.9 & 22.2 & 11.4 \\
iqr 1 & 30.5 & 20.7 & 33.4 & 24.7 & 55.6 & 39.3 & 40.8 & 28.1 & 53.6 & 35.7 & 41.4 & 30.0 \\
med & 36.1 & 31.8 & 42.5 & 35.6 & 61.4 & 53.2 & 49.3 & 36.9 & 61.3 & 49.4 & 48.5 & 37.4 \\
iqr 3 & 44.9 & 44.1 & 47.5 & 45.7 & 69.9 & 63.8 & 57.9 & 49.6 & 70.1 & 60.0 & 58.9 & 46.4 \\
max & 75.0 & 60.0 & 60.6 & 64.6 & 86.9 & 78.5 & 79.6 & 61.9 & 82.8 & 78.1 & 78.4 & 60.7 \\
\hline $\mathrm{n}$ & 28 & 25 & 34 & 26 & 27 & 23 & 33 & 23 & 27 & 23 & 31 & 27 \\
\hline
\end{tabular}

\begin{tabular}{|c|c|c|c|c|c|c|c|c|c|c|c|c|}
\hline & \multicolumn{12}{|c|}{ Spatial hearing } \\
\hline & \multicolumn{4}{|c|}{ Baseline } & \multicolumn{4}{|c|}{3 months } & \multicolumn{4}{|c|}{6 months } \\
\hline & CI & BCD & CROS & No $\mathrm{Tr}$ & CI & BCD & CROS & No Tr & CI & BCD & CROS & No $T r$ \\
\hline $\min$ & 4.1 & 7.5 & 7.9 & 6.5 & 5.6 & 15.3 & 8.6 & 6.9 & 14.1 & 12.5 & 13.5 & 8.2 \\
\hline iqr 1 & 14.9 & 17.6 & 18.7 & 16.0 & 38.1 & 26.9 & 27.6 & 14.4 & 42.1 & 23.4 & 21.6 & 15.7 \\
\hline med & 22.9 & 21.8 & 25.9 & 28.2 & 51.3 & 36.2 & 39.3 & 30.0 & 55.9 & 37.4 & 38.4 & 26.3 \\
\hline iqr 3 & 35.6 & 30.5 & 40.0 & 41.6 & 64.7 & 53.5 & 52.4 & 40.8 & 65.3 & 56.8 & 44.8 & 40.2 \\
\hline $\max$ & 44.2 & 52.9 & 71.8 & 76.6 & 74.0 & 69.1 & 77.0 & 83.8 & 77.4 & 76.9 & 73.5 & 71.8 \\
\hline $\mathrm{n}$ & 28 & 25 & 34 & 26 & 27 & 23 & 33 & 23 & 27 & 23 & 31 & 27 \\
\hline
\end{tabular}

\begin{tabular}{|c|c|c|c|c|c|c|c|c|c|c|c|c|}
\hline & \multicolumn{12}{|c|}{ Qualities of hearing } \\
\hline & \multicolumn{4}{|c|}{ Baseline } & \multicolumn{4}{|c|}{3 months } & \multicolumn{4}{|c|}{6 months } \\
\hline & CI & BCD & CROS & No $\mathrm{Tr}$ & CI & BCD & CROS & No $T r$ & CI & BCD & CROS & No $\mathrm{Tr}$ \\
\hline $\min$ & 32.8 & 21.2 & 41.9 & 25.7 & 33.1 & 42.4 & 47.6 & 21.9 & 44.4 & 23.7 & 46.1 & 28.1 \\
\hline iqr 1 & 52.2 & 46.1 & 57.1 & 48.9 & 60.0 & 54.2 & 61.3 & 46.6 & 60.5 & 48.9 & 59.9 & 51.3 \\
\hline med & 58.5 & 54.9 & 64.4 & 61.6 & 68.9 & 66.5 & 68.9 & 54.8 & 68.3 & 60.6 & 65.1 & 59.6 \\
\hline iqr 3 & 66.3 & 65.3 & 71.2 & 65.6 & 73.4 & 73.5 & 74.7 & 68.6 & 73.8 & 71.6 & 73.5 & 67.3 \\
\hline $\max$ & 75.5 & 80.8 & 80.8 & 76.9 & 81.7 & 90.9 & 88.9 & 77.8 & 83.2 & 94.7 & 85.6 & 79.1 \\
\hline $\mathrm{n}$ & 28 & 25 & 34 & 26 & 27 & 23 & 33 & 23 & 27 & 23 & 31 & 27 \\
\hline
\end{tabular}

\section{Legend:}

Results on the SSQ questionnaire per subscale. Possible range of score 0.0-100.0. The responses were given on a visual analogue scale; a higher score reflects a better performance.

$\mathrm{CI}=$ cochlear implant, $\mathrm{BCD}=$ Bone Conduction Device, $\mathrm{CROS}=$ Contralateral Routing of Sound hearing aid, No $\mathrm{Tr}=$ No treatment

Minimum (min) and maximum (max) values are presented, as well as first and third quartiles (iqr 1 and iqr 3, respectively), medians (med) and number of measurements (n). 
Table 5: APHAB questionnaire

\begin{tabular}{|c|c|c|c|c|c|c|c|c|c|c|c|c|}
\hline & \multicolumn{12}{|c|}{ EC } \\
\hline & \multicolumn{4}{|c|}{ Baseline } & \multicolumn{4}{|c|}{3 months } & \multicolumn{4}{|c|}{6 months } \\
\hline & CI & BCD & CROS & No $\mathrm{Tr}$ & CI & BCD & CROS & No $\mathrm{Tr}$ & CI & BCD & CROS & No $\mathrm{Tr}$ \\
\hline $\min$ & 0.11 & 0.07 & 0.09 & 0.08 & 0.05 & 0.01 & 0.05 & 0.11 & 0.05 & 0.01 & 0.07 & 0.12 \\
\hline iqr 1 & 0.24 & 0.23 & 0.21 & 0.18 & 0.10 & 0.16 & 0.18 & 0.18 & 0.12 & 0.14 & 0.17 & 0.23 \\
\hline med & 0.33 & 0.38 & 0.27 & 0.33 & 0.15 & 0.26 & 0.21 & 0.28 & 0.15 & 0.26 & 0.25 & 0.29 \\
\hline iqr 3 & 0.50 & 0.54 & 0.40 & 0.45 & 0.25 & 0.31 & 0.29 & 0.46 & 0.23 & 0.31 & 0.29 & 0.46 \\
\hline $\max$ & 0.56 & 0.93 & 0.62 & 0.83 & 0.52 & 0.40 & 0.50 & 0.79 & 0.37 & 0.40 & 0.56 & 0.79 \\
\hline$n$ & 28 & 25 & 34 & 26 & 24 & 20 & 32 & 24 & 26 & 22 & 30 & 27 \\
\hline
\end{tabular}

\begin{tabular}{lccccccccccccc}
\hline \multicolumn{10}{c}{ BN } \\
\hline \multicolumn{10}{c}{ Baseline } & \multicolumn{10}{c}{ 3 months } \\
\hline & CI & BCD & CROS & No Tr & CI & BCD & CROS & No Tr & CI & BCD & CROS & No Tr \\
\hline min & 0.48 & 0.35 & 0.39 & 0.35 & 0.07 & 0.27 & 0.19 & 0.38 & 0.07 & 0.18 & 0.12 & 0.31 \\
iqr 1 & 0.65 & 0.64 & 0.62 & 0.59 & 0.25 & 0.39 & 0.46 & 0.56 & 0.28 & 0.42 & 0.46 & 0.57 \\
med & 0.74 & 0.73 & 0.72 & 0.71 & 0.46 & 0.58 & 0.59 & 0.71 & 0.41 & 0.52 & 0.59 & 0.77 \\
iqr 3 & 0.77 & 0.85 & 0.84 & 0.79 & 0.59 & 0.68 & 0.68 & 0.77 & 0.48 & 0.63 & 0.76 & 0.84 \\
max & 0.99 & 0.95 & 0.93 & 0.95 & 0.83 & 0.89 & 0.89 & 0.99 & 0.83 & 0.91 & 0.87 & 0.97 \\
\hline $\mathrm{n}$ & 28 & 25 & 34 & 26 & 24 & 20 & 32 & 24 & 26 & 22 & 30 & 27 \\
\hline
\end{tabular}

\begin{tabular}{lccccccccccccc}
\hline \multicolumn{10}{c}{ RV } \\
\hline \multicolumn{10}{c}{ Baseline } & \multicolumn{10}{c}{ 3 months } \\
\hline & CI & BCD & CROS & No Tr & CI & BCD & CROS & No Tr & CI & BCD & CROS & No Tr \\
\hline min & 0.29 & 0.10 & 0.13 & 0.08 & 0.12 & 0.16 & 0.09 & 0.12 & 0.05 & 0.01 & 0.09 & 0.16 \\
iqr 1 & 0.43 & 0.46 & 0.44 & 0.42 & 0.18 & 0.29 & 0.24 & 0.34 & 0.18 & 0.30 & 0.29 & 0.42 \\
med & 0.62 & 0.60 & 0.55 & 0.53 & 0.29 & 0.38 & 0.30 & 0.52 & 0.28 & 0.39 & 0.33 & 0.52 \\
iqr 3 & 0.67 & 0.75 & 0.65 & 0.70 & 0.42 & 0.57 & 0.42 & 0.61 & 0.35 & 0.53 & 0.45 & 0.68 \\
max & 0.87 & 0.95 & 0.91 & 0.91 & 0.69 & 0.69 & 0.69 & 0.91 & 0.54 & 0.75 & 0.71 & 0.89 \\
\hline $\mathrm{n}$ & 28 & 25 & 34 & 26 & 24 & 20 & 32 & 24 & 26 & 22 & 30 & 27 \\
\hline
\end{tabular}

\begin{tabular}{|c|c|c|c|c|c|c|c|c|c|c|c|c|}
\hline & \multicolumn{12}{|c|}{ AS } \\
\hline & \multicolumn{4}{|c|}{ Baseline } & \multicolumn{4}{|c|}{3 months } & \multicolumn{4}{|c|}{6 months } \\
\hline & CI & BCD & CROS & No $\operatorname{Tr}$ & CI & BCD & CROS & No $\operatorname{Tr}$ & CI & BCD & CROS & No $\operatorname{Tr}$ \\
\hline $\min$ & 0.11 & 0.03 & 0.07 & 0.08 & 0.03 & 0.05 & 0.03 & 0.11 & 0.08 & 0.03 & 0.07 & 0.11 \\
\hline iqr 1 & 0.37 & 0.31 & 0.36 & 0.44 & 0.18 & 0.23 & 0.29 & 0.34 & 0.17 & 0.25 & 0.37 & 0.30 \\
\hline med & 0.54 & 0.50 & 0.50 & 0.60 & 0.35 & 0.38 & 0.42 & 0.52 & 0.31 & 0.36 & 0.46 & 0.62 \\
\hline iqr 3 & 0.65 & 0.64 & 0.61 & 0.74 & 0.52 & 0.54 & 0.50 & 0.68 & 0.46 & 0.48 & 0.59 & 0.73 \\
\hline $\max$ & 0.87 & 0.79 & 0.83 & 0.97 & 0.83 & 0.70 & 0.69 & 0.97 & 0.85 & 0.81 & 0.81 & 0.97 \\
\hline $\mathrm{n}$ & 28 & 25 & 34 & 26 & 24 & 20 & 32 & 24 & 26 & 22 & 30 & 27 \\
\hline
\end{tabular}




\section{Legend:}

Results on the APHAB questionnaire per subscale (ease of communication (EC), listening under reverberant conditions (RV), listening in background noise (BN), and aversiveness of sounds (AS)). Possible range of score 0.0-100.0 per subscale. A lower score reflects less problems in daily life.

$\mathrm{CI}=$ cochlear implant, $\mathrm{BCD}=$ Bone Conduction Device, $\mathrm{CROS}=$ Contralateral Routing of Sound hearing aid, No $\operatorname{Tr}=$ No treatment

Minimum ( $\min )$ and maximum $(\max )$ values are presented, as well as first and third quartiles (iqr 1 and iqr 3, respectively), medians (med) and number of measurements (n). 
Table 6: GBI

\begin{tabular}{|c|c|c|c|c|c|c|}
\hline & \multicolumn{6}{|c|}{ General } \\
\hline & \multicolumn{3}{|c|}{3 months } & \multicolumn{3}{|c|}{6 months } \\
\hline & CI & BCD & CROS & CI & BCD & CROS \\
\hline $\min$ & -25.00 & 0.00 & -18.18 & -75.00 & -8.33 & -29.17 \\
\hline iqr 1 & 14.58 & 8.33 & 4.17 & 10.80 & 8.33 & 10.80 \\
\hline med & 29.17 & 20.83 & 20.83 & 25.00 & 20.83 & 20.83 \\
\hline iqr 3 & 41.67 & 33.33 & 33.33 & 33.33 & 29.17 & 29.17 \\
\hline $\max$ & 66.67 & 54.17 & 54.17 & 79.17 & 77.27 & 58.33 \\
\hline \multirow[t]{4}{*}{$\mathrm{n}$} & 27 & 23 & 31 & 27 & 23 & 31 \\
\hline & \multicolumn{6}{|c|}{ Social support } \\
\hline & \multicolumn{3}{|c|}{3 months } & \multicolumn{3}{|c|}{6 months } \\
\hline & CI & BCD & CROS & CI & BCD & CROS \\
\hline $\min$ & 0.00 & -33.33 & -16.67 & 0.00 & -16.67 & -50.00 \\
\hline iqr 1 & 0.00 & 0.00 & 0.00 & 0.00 & 0.00 & 0.00 \\
\hline med & 0.00 & 0.00 & 0.00 & 0.00 & 0.00 & 0.00 \\
\hline iqr 3 & 25.00 & 0.00 & 0.00 & 8.33 & 0.00 & 0.00 \\
\hline $\max$ & 50.00 & 33.33 & 16.67 & 33.33 & 33.33 & 33.33 \\
\hline \multirow[t]{4}{*}{$\mathrm{n}$} & 27 & 23 & 31 & 27 & 23 & 31 \\
\hline & \multicolumn{6}{|c|}{ Physical health } \\
\hline & \multicolumn{3}{|c|}{3 months } & \multicolumn{3}{|c|}{6 months } \\
\hline & CI & BCD & CROS & CI & BCD & CROS \\
\hline $\min$ & -50.00 & -33.33 & -33.33 & -33.33 & -33.33 & -50.00 \\
\hline iqr 1 & 0.00 & 0.00 & 0.00 & 0.00 & 0.00 & 0.00 \\
\hline med & 0.00 & 0.00 & 0.00 & 0.00 & 0.00 & 0.00 \\
\hline iqr 3 & 0.00 & 0.00 & 0.00 & 0.00 & 0.00 & 0.00 \\
\hline $\max$ & 16.67 & 0.00 & 16.67 & 16.67 & 16.67 & 0.00 \\
\hline $\mathrm{n}$ & 27 & 23 & 31 & 27 & 23 & 31 \\
\hline
\end{tabular}

\section{Legend:}

Results on the Glasgow Benefit Inventory (GBI) per subscale (general, social support, physical health). Possible range of score -100.0 to 100.0 per subscale. A higher score reflects more benefit from the intervention, a negative score reflects disadvantage from the intervention. The only statistically significant difference between-groups was the difference between the CI and CROS groups at 3 months follow-up $(\mathrm{p}=0.018)$.

$\mathrm{CI}=$ cochlear implant, $\mathrm{BCD}=$ Bone Conduction Device, $\mathrm{CROS}=$ Contralateral Routing of Sound hearing aid

Minimum (min) and maximum (max) values are presented, as well as first and third quartiles (iqr 1 and iqr 3, respectively), medians (med) and number of measurements (n). 


\section{Sources of Funding}

This study is partly funded by Cochlear Ltd. as an unrestricted research grant. By research contract, Cochlear Ltd. did not have influence on the study design, data collection, analysis, data interpretation, and publication.

\section{Conflicts of Interest}

The authors have no conflicts of interest to declare.

\section{Author contributions}

Contributed to the design of the study: JPMP, GAvZ, WG, ALS.

Contributed to the acquisition of data: JPMP, JAAvH, AWW.

Contributed to the analysis of data: JPMP, JAAvH, AWW.

Contributed to the interpretation of data: all authors.

Contributed to drafting the manuscript: JPMP, JAAvH, AWW.

Contributed to revising the manuscript critically for important intellectual content: all authors.

Final approval of the version to be published: all authors.

Authors JAAvH and AWW contributed equally. 


\section{REFERENCES}

1. Van de Heyning P, Távora-Vieira D, Mertens G, Van Rompaey V, Rajan GP, Müller J, Hempel JM, Leander D, Polterauer D, Marx M, Usami SI, Kitoh R, Miyagawa M, Moteki H, Smilsky K, Baumgartner WD, Keintzel TG, Sprinzl GM, Wolf-Magele A, Arndt S, et al. Towards a Unified Testing Framework for Single-Sided Deafness Studies: A Consensus Paper. Audiol Neurootol 2016;21(6):391-398.

2. Zeitler DM, Dorman MF. Cochlear Implantation for Single-Sided Deafness: A New Treatment Paradigm. J Neurol Surg B Skull Base 2019;80(2):178-186.

3. Dunn CC, Tyler RS, Oakley S, Gantz BJ, Noble W. Comparison of speech recognition and localisation performance in bilateral and unilateral cochlear implant users matched on duration of deafness and age at implantation. Ear Hear 2008;29:325-359.

4. Van Wanrooij MM, Van Opstal AJ. Contribution of Head Shadow and Pinna Cues to Chronic Monaural Sound Localisation. J Neurosc 2004;24:4163-4171.

5. Van de Heyning P, Vermeire K, Diebl M, Nopp P, Anderson I, De Ridder D. Incapacitating unilateral tinnitus in single-sided deafness treated by cochlear implantation. Annals of Otol, Rhinol and Laryngol 2008;117(9):645-652.

6. Wie OB, Pripp AH, Tvete O. Unilateral deafness in adults: effects on communication and social interaction. Annals of Otol Rhinol Laryngol 2010;119(11):772-781.

7. Olze H, Szczepek AJ, Haupt H, Förster U, Zirke N, Gräbel S, Mazurek B. Cochlear implantation has a positive influence on quality of life, tinnitus, and psychological comorbidity. Laryngoscope 2011;121(10):2220-2227.

8. Lucas L, Katiri R, Kitterick PT. The psychological and social consequences of singlesided deafness in adulthood. Int J Audiol 2018;57(1):21-30.

9. Peters JPM, Smit AL, Stegeman I, Grolman W. Review: Bone Conduction Devices and Contralateral Routing of Sound Systems in Single-Sided Deafness. Laryngoscope 2015a;125:218-226. 
10. Mertens G, De Bodt M, Van de Heyning P. Evaluation of Long-Term Cochlear Implant Use in Subjects With Acquired Unilateral Profound Hearing Loss: Focus on Binaural Auditory Outcomes. Ear Hear 2017;38(1):117-125.

11. Galvin JJ 3rd, Fu QJ, Wilkinson EP, Mills D, Hagan SC, Lupo JE, Padilla M, Shannon RV. Benefits of Cochlear Implantation for Single-Sided Deafness: Data From the House Clinic-University of Southern California-University of California, Los Angeles Clinical Trial. Ear Hear 2019;40(4):766-781.

12. Kurz A, Rak K, Hagen R, Ehrmann-Müller D. Evaluating the Decision for Cochlear Implantation in Individuals With Single-Sided Deafness (SSD); Implementing the SSD Consensus Protocol Into Clinical Routine. Otol Neurotol 2020;41(6):727-735.

13. Arts RA, George EL, Janssen M, Griessner A, Zierhofer C, Stokroos RJ. Tinnitus Suppression by Intracochlear Electrical Stimulation in Single Sided Deafness - A Prospective Clinical Trial: Follow-Up. PLoS One 2016;11(4):e0153131.

14. Arts RA, George EL, Stokroos RJ, Vermeire K. Review: cochlear implants as a treatment of tinnitus in single-sided deafness. Curr Opin Otolaryngol Head Neck Surg 2012;20(5):398-403.

15. Van Zon A, Peters JPM, Smit AL, Stegeman I, Grolman W. Cochlear Implantation for Patients with Single-Sided Deafness or Asymmetrical Hearing Loss: a Systematic Review of the Evidence. Otol Neurotol 2015;36:209-219.

16. Peter N, Liyanage N, Pfiffner F, Huber A, Kleinjung T. The Influence of Cochlear Implantation on Tinnitus in Patients with Single-Sided Deafness: A Systematic Review. Otolaryngol Head Neck Surg 2019;161(4):576-588.

17. Muigg F, Bliem HR, Kühn H, Seebacher J, Holzner B, Weichbold VW. Cochlear implantation in adults with single-sided deafness: generic and disease-specific long-term quality of life. Eur Arch Otorhinolaryngol 2020;277(3):695-704.

18. Vlastarakos PV, Nazos K, Tavoulari EF, Nikolopoulos TP. Cochlear implantation for single-sided deafness: the outcomes. An evidence based approach. Eur Arch Otorhinolaryngol 2014;271(8):2119-2126. 
19. Kitterick PT, Smith SN, Lucas L. Hearing Instruments for Unilateral Severe-to-Profound Sensorineural Hearing Loss in Adults: A Systematic Review and Meta-Analysis. Ear Hear 2016;37(5):495-507.

20. Peters JPM, Van Zon A, Smit AL, Van Zanten GA, De Wit GA, Stegeman I, Grolman W. CINGLE-trial: Cochlear Implantation for siNGLE-sided deafness, a Randomised controlled trial and economic evaluation. BMC Ear, Nose and Throat Disorders 2015b;15:3.

21. Schulz KF, Altman DG, Moher D for the CONSORT group. CONSORT 2010 statement: updated guidelines for reporting parallel group Randomized trials. BMJ 2010;340:c332.

22. Smulders YE, Rinia AB, Pourier VEC, Van Zon A, Van Zanten GA, Stegeman I, Scherf FWAC, Smit AL, Topsakal V, Tange RA, Grolman W. Validation of the U-STARR with the $\mathrm{AB}$-York crescent of sound, a new instrument to evaluate speech intelligibility in noise and spatial hearing skills. Audiol Neurotol Extra 2015;5:1-10.

23. Kitterick PT, Lovett RE, Goman AM, Summerfield AQ. The AB-York crescent of sound: an apparatus for assessing spatial-listening skills in children and adults. Cochlear Implants Int 2011;12(3):164-169.

24. Meeus O, Blaivie C, Van de Heyning P. Validation of the Dutch and the French version of the Tinnitus Questionnaire. B-ENT 2007;3(Suppl 7):11-17.

25. Goebel G, Hiller W. The tinnitus questionnaire. A standard instrument for grading the degree of tinnitus. Results of a multicenter study with the tinnitus questionnaire. HNO 1994;42(3):166-172.

26. Newman CW, Jacobson GP, Spitzer JB. Development of the tinnitus handicap inventory. Arch Otolaryngol Head Neck Surg 1996;122:143-148.

27. Gatehouse S, Noble W. The speech, spatial and qualities of hearing scale (SSQ). Inter J Audio 2004;43:85-99.

28. Cox RM, Alexander GC. The abbreviated profile for hearing aid benefit. Ear Hear $1998 ; 16: 176-186$. 
29. Robinson K, Gatehouse S, Browning GG. Measuring patient benefit from otorhinolaryngological surgery and therapy. Ann Otol Rhinol Laryngol 1996;105:415422.

30. Arndt S, Aschendorff A, Laszig R, Beck R, Schild C, Kroeger S, Ihorst G, Wesarg T. Comparison of pseudobinaural hearing to real binaural hearing rehabilitation after cochlear implantation in patients with unilateral deafness and tinnitus. Otol Neurotol 2010;32(1):39-47.

31. Vermeire K, Van de Heyning P. Binaural hearing after cochlear implantation in subjects with unilateral sensorineural deafness and tinnitus. Audiol Neurotol 2009;14:163-171.

32. Dorman MF, Zeitler D, Cook SJ, Loiselle L, Yost WA, Wanna GB, Gifford RH. Interaural level difference cues determine sound source localisation by single-sided deaf patients fit with a cochlear implant. Audiol Neurotol 2015;20(3):183-188.

33. Sullivan CB, Al-Qurayshi Z, Zhu V, Liu A, Dunn C, Gantz BJ, Hansen MR. Long-term audiologic outcomes after cochlear implantation for single-sided deafness. Laryngoscope 2020;130(7):1805-1811.

34. Yost WA, Loiselle L, Dorman MF, Burns J, Brown CA. Sound source localisation of filtered noises by listeners with normal hearing: a statistical analysis. J Acoust Soc Am 2013;133:2876-2882.

35. Agterberg MJH, Snik AFM, Van de Goor RMG, Hol MKS, Van Opstal AJ. Soundlocalisation performance of patients with single-sided deafness is not improved when listening with a bone-conduction device. Hear Res 2019;372:62-68.

36. Choi JE, Ma SM, Park H, Cho YS, Hong SH, Moon IJ. A comparison between wireless CROS/BiCROS and soft-band BAHA for patients with unilateral hearing loss. PLoS One 2019;14(2):e0212503.

37. Távora-Vieira D, Marino R, Krishnaswamy J, Kuthbutheen J, Rajan GP. Cochlear implantation for unilateral deafness with and without tinnitus: A case series. Laryngoscope 2013;123(5):1251-1255. 
38. Ramos Á, Polo R, Masgoret E, Artiles O, Lisner I, Zaballos ML, Moreno C, Osorio Á. Cochlear implant in patients with sudden unilateral sensorineural hearing loss and associated tinnitus. Acta Otorrinolaringol Esp 2012;63(1):15-20.

39. Poncet-Wallet C, Mamelle E, Godey B, Truy E, Guevara N, Ardoint M, Gnansia D, Hoen M, Saaï S, Mosnier I, Lescanne E, Bakhos D, Vincent C. Prospective Multicentric Followup Study of Cochlear Implantation in Adults With Single-Sided Deafness: Tinnitus and Audiological Outcomes. Otol Neurotol 2020;41(4):458-466.

40. Indeyeva YA, Diaz A, Imbrey T, Gao G, Coelho DH. Tinnitus management with percutaneous osseointegrated auditory implants for unilateral sensorineural hearing loss. Am J Otolaryngol 2015;36(6):810-813.

41. Dillon MT, Buss E, Rooth MA, King ER, Deres EJ, Buchman CA, Pillsbury HC, Brown KD. Effect of Cochlear Implantation on Quality of Life in Adults with Unilateral Hearing Loss. Audiol Neurotol 2017;22(4-5):259-271.

42. Wendrich AW, Kroese TE, Peters JPM, Cattani G, Grolman W. Systematic Review on the Trial Period for Bone Conduction Devices in Single-Sided Deafness: Rates and Reasons for Rejection. Otol Neurotol 2017;38(5):632-641.

43. Peter N, Kleinjung T, Probst R, Hemsley C, Veraguth D, Huber A, Caversaccio M, Kompis M, Mantokoudis G, Senn P, Wimmer W. Cochlear implants in single-sided deafness clinical results of a Swiss multicentre study. Swiss Med Wkly 2019;149:w20171.

44. Cohen SM, Svirsky MA. Duration of unilateral auditory deprivation is associated with reduced speech perception after cochlear implantation: A single-sided deafness study. Cochlear Implants Int 2019;20(2):51-56.

45. Kurz A, Grubenbecher M, Rak K, Hagen R, Kühn H. The impact of etiology and duration of deafness on speech perception outcomes in SSD patients. Eur Arch Otorhinolaryngol 2019;276(12):3317-3325.

46. Boutron I, Tubach F, Giraudeau B, Ravaud P. Blinding was judged more difficult to achieve and maintain in nonpharmacologic than pharmacologic trials. J Clin Epidemiol 
2004;57(6):543-550.

47. Kamalski DM, Hoekstra CE, van Zanten BG, Grolman W, Rovers MM. Measuring disease-specific health-related quality of life to evaluate treatment outcomes in tinnitus patients: a systematic review. Otolaryngol Head Neck Surg 2010;143:181-185.

48. Meikle MB, Henry JA, Griest SE, Stewart BJ, Abrams HB, McArdle R, Myers PJ, Newman CW, Sandridge S, Turk DC, Folmer RL, Frederick EJ, House JW, Jacobson GP, Kinney SE, Martin WH, Nagler SM, Reich GE, Searchfield G, Sweetow R, et al. The tinnitus functional index: development of a new clinical measure for chronic intrusive tinnitus. Ear Hear 2012;33(2):153-176.

49. Zeman F, Koller M, Schecklmann M, Langguth B, Landgrebe M, TRI database study group. Tinnitus assessment by means of standardized self-report questionnaires: psychometric properties of the Tinnitus Questionnaire (TQ), the Tinnitus Handicap Inventory (THI), and their short versions in an international and multi-lingual sample. Health Qual Life Outcomes 2012;10:128.

50. Ontario Health (Quality). Implantable devices for single-sided deafness and conductive or mixed hearing loss: a health technology assessment. Ont Health Technol Assess Ser (Internet) 2020;20(1):1-165. Available from: https://www.hqontario.ca/Evidenceto-Improve-Care/Health-Technology-Assessment/Reviews-And-Recommendations/ $\underline{\text { Implantable-Devices-for-Single-Sided-Deafness-and-Conductive-or-Mixed-Hearing- }}$ Loss, accessed April 1st, 2020.

51. Kitterick PT, O’Donoghue GM, Edmondson-Jones M, Marshall A, Jeffs E, Craddock L, Riley A, Green K, O’Driscoll M, Jiang D, Nunn T, Saeed S, Aleksy W, Seeber BU. Comparison of the benefits of cochlear implantation versus contra-lateral routing of signal hearing aids in adult patients with single-sided deafness: study protocol for a prospective within-subject longitudinal trial. BMC Ear Nose Throat Disord 2014;14:7.

52. Marx M, Costa N, Lepage B, Taoui S, Molinier L, Deguine O, Fraysse B. Cochlear implantation as a treatment for single-sided deafness and asymmetric hearing loss: a 
randomized controlled evaluation of cost-utility. BMC Ear Nose Throat Disord 2019;19:1. 53. Katiri R, Hall DA, Buggy N, Hogan N, Horobin A, Van de Heyning P, Firszt JB, Bruce IA, Kitterick PT. Core Rehabilitation Outcome Set for Single Sided Deafness (CROSSSD) study: protocol for an international consensus on outcome measures for single sided deafness interventions using a modified Delphi survey. Trials 2020;21(1):238. 



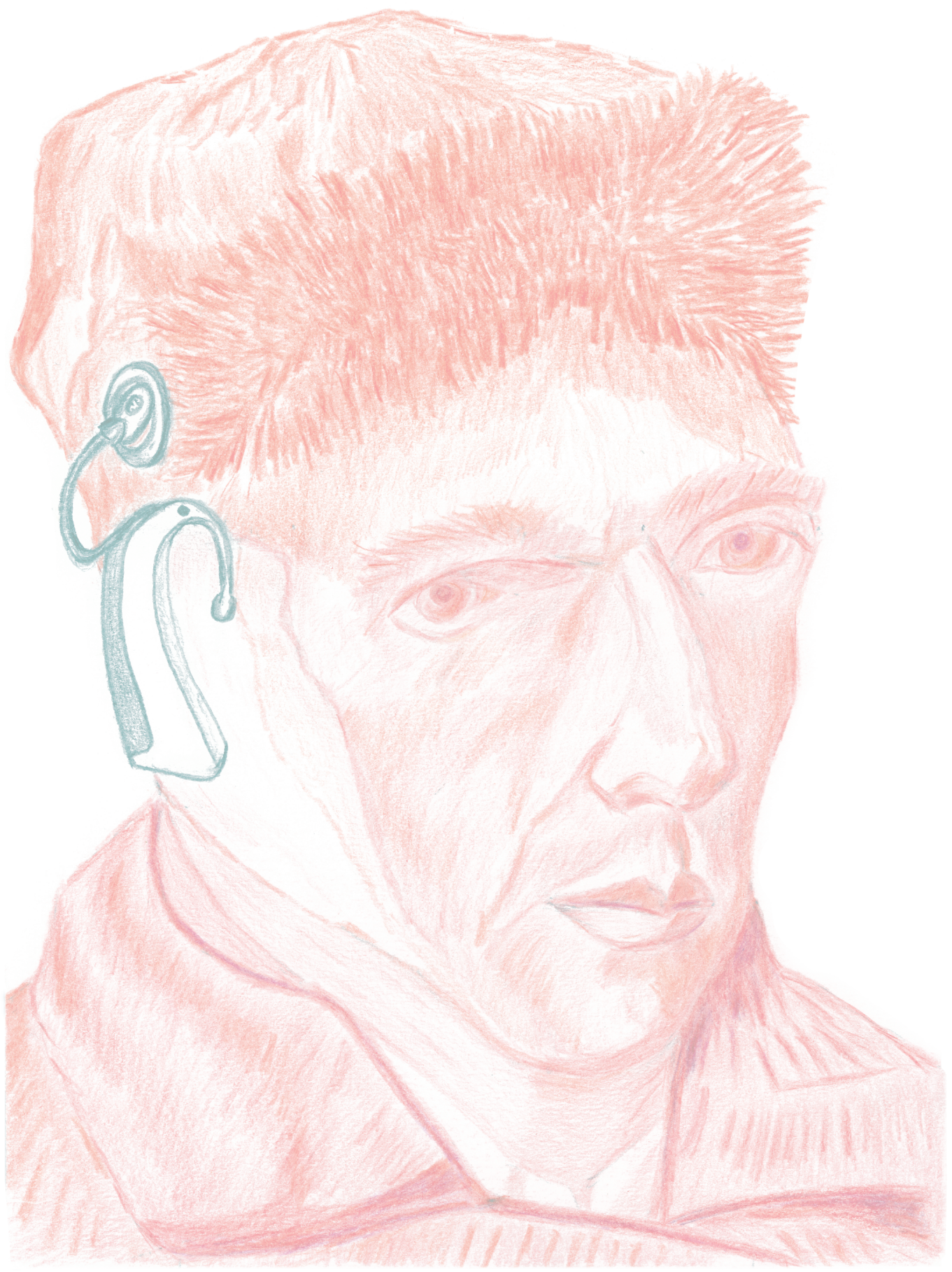




\section{Part III}

\section{Vocoder and pitch match experiments}




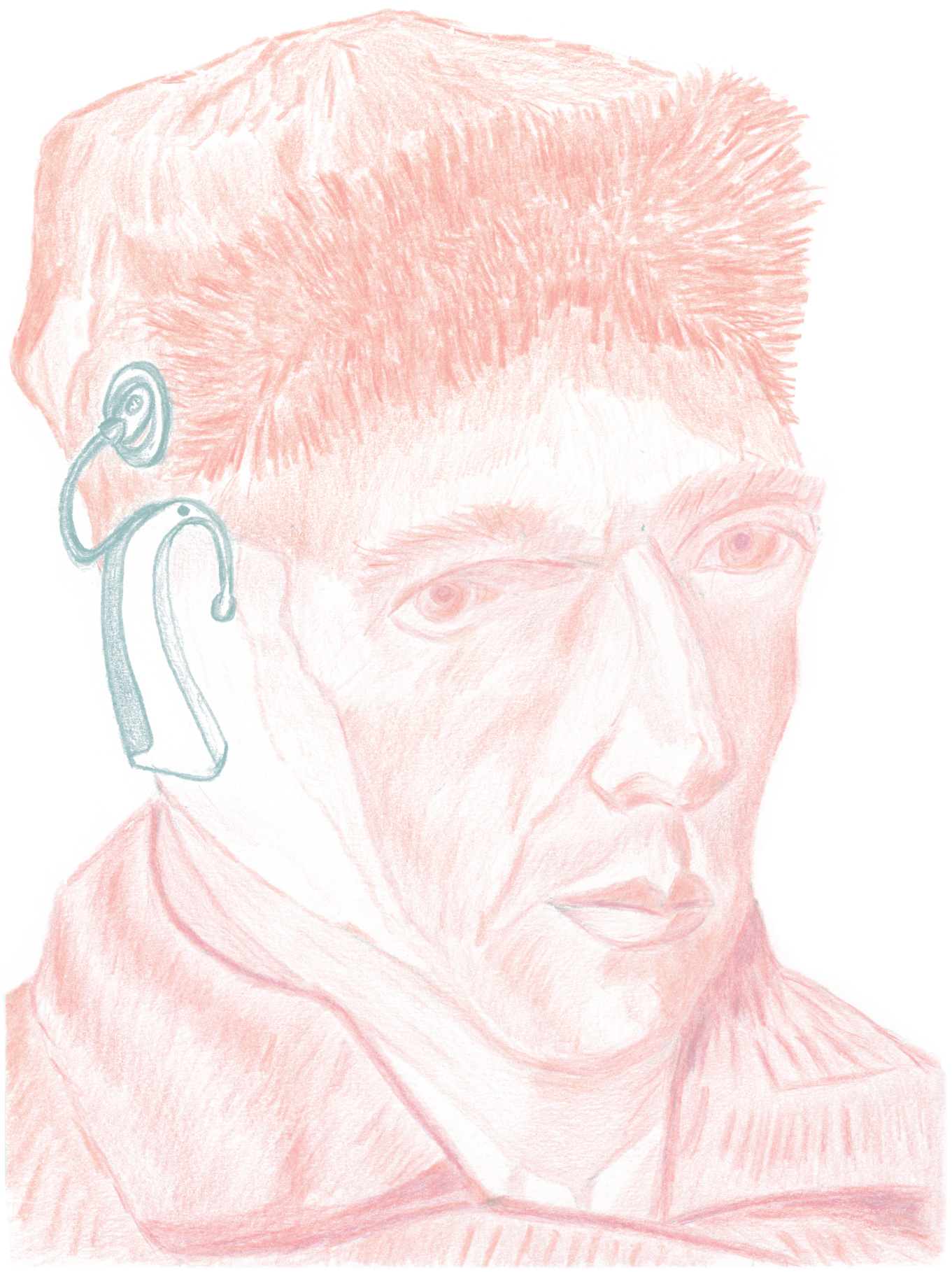




\section{6}

\section{The Sound of a Cochlear Implant Investigated in Patients with}

Single-Sided Deafness and a Cochlear Implant

Jeroen P.M. Peters, Anne W. Wendrich, Ruben H.M. van Eijl, Koenraad S. Rhebergen,

Huib Versnel, Wilko Grolman. 


\section{ABSTRACT}

\section{Hypothesis}

A cochlear implant (CI) restores hearing in patients with profound sensorineural hearing loss by electrical stimulation of the auditory nerve. It is unknown how this electrical stimulation sounds.

\section{Background}

Patients with single-sided deafness (SSD) and a CI form a unique population, since they can compare the sound of their CI to simulations of the CI sound played to their non-implanted ear.

\section{Methods}

We tested six stimuli (speech and music) in 10 SSD patients implanted with a CI (Cochlear Ltd). Patients listened to the original stimulus with their CI ear while their non-implanted ear was masked. Subsequently, patients listened to two CI simulations, created with a vocoder, with their non-implanted ear alone. They selected the CI simulation with greatest similarity to the sound as perceived by their CI ear and they graded similarity on a 1-10 scale. We tested three vocoders: two known from literature, and one supplied by Cochlear Ltd. Two carriers (noise, sine) were tested for each vocoder.

\section{Results}

Carrier noise and the vocoders from literature were most often selected as best match to the sound as perceived by the CI ear. However, variability in selections was substantial both between patients and within patients between sound samples. The average grade for similarity was 6.8 for speech stimuli and 6.3 for music stimuli. 


\section{Conclusions}

We obtained a fairly good impression of what a CI can sound like for SSD patients. This knowledge may help to better inform and educate patients and family members about the sound of a CI. 


\section{INTRODUCTION}

A cochlear implant (CI) provides auditory information to the patient deafened by profound damage of the cochlea by electrically stimulating the auditory nerve. The electrode array of a CI has between 12 and 22 electrode contacts, which is surgically implanted in the cochlea to distribute auditory information tonotopically. Although the number of channels in a CI is much smaller than the 3000-4000 inner hair cells in a healthy cochlea, CI patients generally reach advanced speech perception and many postlingually deaf patients can even understand speech through a phone. ${ }^{1}$ As expected considering the artificial processing by the device, most patients do not find the sound of their CI natural. Instead, they describe the sound as "robotic", "metallic" or "electrical". The unnatural sound quality contributes to poor music appreciation. $^{2}$

For the last 20 years, vocoders ('voice encoders') have been used to simulate CIs. ${ }^{3}$ Normal-hearing $(\mathrm{NH})$ subjects have been tested as if they were CI patients by presenting them vocoded stimuli. Testing $\mathrm{NH}$ subjects has the advantage that confounding factors associated with CI patients, such as neuron survival patterns, are absent. ${ }^{4}$ In these experiments with NH subjects, speech perception, speaker gender identification and sound localization were poorer with the vocoded versions of the stimuli compared to the original versions..$^{5-12}$

Although current vocoders mimic the signal processing of a CI, we cannot be sure that the output of these vocoders is similar to the sound that CI users actually perceive. The results of the above-mentioned experiments have greater value if the vocoders that are used approach the sound perceived through a CI. Therefore, these vocoder models must be validated. ${ }^{13} \mathrm{CI}$ patients who acquired bilateral deafness later in life can only describe what their implant sounds like when comparing the CI sound to their memory of acoustic hearing, but they cannot answer this question in real-time. In contrast, patients with single-sided deafness (SSD) and a CI in their deaf ear can compare sounds that they hear with their CI ear to sounds that they hear with their non-implanted ear. Accordingly, in this study, we examined 10 SSD patients with a CI and aimed to answer the research question: "What does 
a CI sound like?". With an answer to this question, we can better inform and educate CI candidates, patients and their family members about the sound of a CI. This knowledge may also influence future developments in CI sound strategies.

\section{MATERIALS AND METHODS}

\section{Patients}

Ten patients with SSD were implanted with a CI as participants in a trial evaluating three treatment modalities for SSD. ${ }^{14}$ The trial is ethically approved by the Institutional Review Board of the University Medical Center Utrecht (NL45288.041.13). All participants provided written informed consent prior to trial participation. To be eligible for inclusion, patients had to be adults with a minimum duration of deafness (DoD) of 3 months and a maximum of 10 years. Furthermore, their pure tone average threshold (PTA; 500, 1000, 2000, and 4000 $\mathrm{Hz}$ ) in the non-implanted ear was maximum $30 \mathrm{~dB}$ hearing loss (HL) and their PTA in the implanted ear was minimum $70 \mathrm{~dB}$ HL. Table 1 shows all individual patient characteristics.

Table 1. Patient characteristics

\begin{tabular}{lllllllll}
\hline Patient & Gender & $\begin{array}{l}\text { Age at } \\
\text { experiment } \\
\text { (years) }\end{array}$ & $\begin{array}{l}\text { Deaf } \\
\text { Ear }\end{array}$ & $\begin{array}{l}\text { Pure Tone } \\
\text { Average^ } \\
\text { (dB HL) }\end{array}$ & Etiology & $\begin{array}{l}\text { Duration } \\
\text { of Deafness } \\
\text { (years) }\end{array}$ & $\begin{array}{l}\text { Experience } \\
\text { with CI } \\
\text { (months) }\end{array}$ & $\begin{array}{l}\text { CVC } \\
\text { phoneme } \\
\text { score }\end{array}$ \\
\hline Pt 1 & Female & 56.6 & Left & 5.0 & Iatrogenic & 8.6 & 12.0 & $91 \%$ \\
Pt 2 & Female & 55.4 & Left & 7.5 & Sudden deafness & 1.2 & 9.9 & $84 \%$ \\
Pt 3 & Male & 68.2 & Left & $31.3^{*}$ & Labyrinthitis & 3.2 & 8.7 & $48 \%$ \\
Pt 4 & Male & 61.5 & Right & 25.0 & Sudden deafness & 2.0 & 7.8 & $76 \%$ \\
Pt 5 & Male & 58.9 & Right & 13.8 & Unknown & 9.8 & 4.8 & $59 \%$ \\
Pt 6 & Male & 47.4 & Left & 7.5 & Ménière's disease & 4.6 & 3.6 & $83 \%$ \\
Pt 7 & Male & 37.8 & Right & 21.3 & Unknown & 3.1 & 2.6 & $93 \%$ \\
Pt 8 & Male & 49.1 & Right & 3.8 & Ménière's disease & 3.2 & 2.3 & $79 \%$ \\
Pt 9 & Male & 64.4 & Left & 28.8 & Unknown & 4.1 & 3.5 & $94 \%$ \\
Pt 10 & Female & 24.0 & Left & 5.0 & Sudden deafness & 1.6 & 2.1 & $78 \%$ \\
\hline
\end{tabular}

\section{Legend:}

$\wedge$ Pure Tone Average (500, 1000, 2000, and $4000 \mathrm{~Hz}$ ) of the non-implanted ear (dB HL), CVC = Consonant-VowelConsonant phoneme score at 3 months after CI activation for the CI-ear only (non-implanted ear masked with masking noise).

* Patient 3 had a PTA of $30.0 \mathrm{~dB}$ HL at time of inclusion, but his hearing slightly deteriorated, which resulted in a PTA of $31.3 \mathrm{~dB} \mathrm{HL}$ at time of the current experiment. 
Figure 1 depicts the audiograms of the non-implanted ears. All patients were implanted with a Nucleus CI (type CI 422, Cochlear Ltd.) and were fitted with CP920 (patients 7 and 8) or CP910 (all other patients) sound processors. After surgery, the audiological rehabilitation phase was identical to regular clinical care for bilaterally deaf CI patients. Additionally, patients were instructed to practice speech perception with the CI ear only, by masking the non-implanted ear with speech-shaped noise via an insert earphone for at least three sessions of at least ten minutes per day. The noise had a long-term average spectrum based on the Dutch consonant-vowel-consonant (CVC) corpus. ${ }^{15}$ The loudness of the masking noise varied per patient, but we ensured that adequate masking was achieved and the original sound was perceived with the CI ear only. Moreover, in standard clinical follow-up the CVC phoneme score was measured for the CI ear only, again by masking the non-implanted ear. It is important to highlight that the patients were thus accustomed to hearing the masking noise in their non-implanted ear while practicing listening with their CI ear alone.

Figure 1. Pure tone audiometry of non-implanted ears of patients

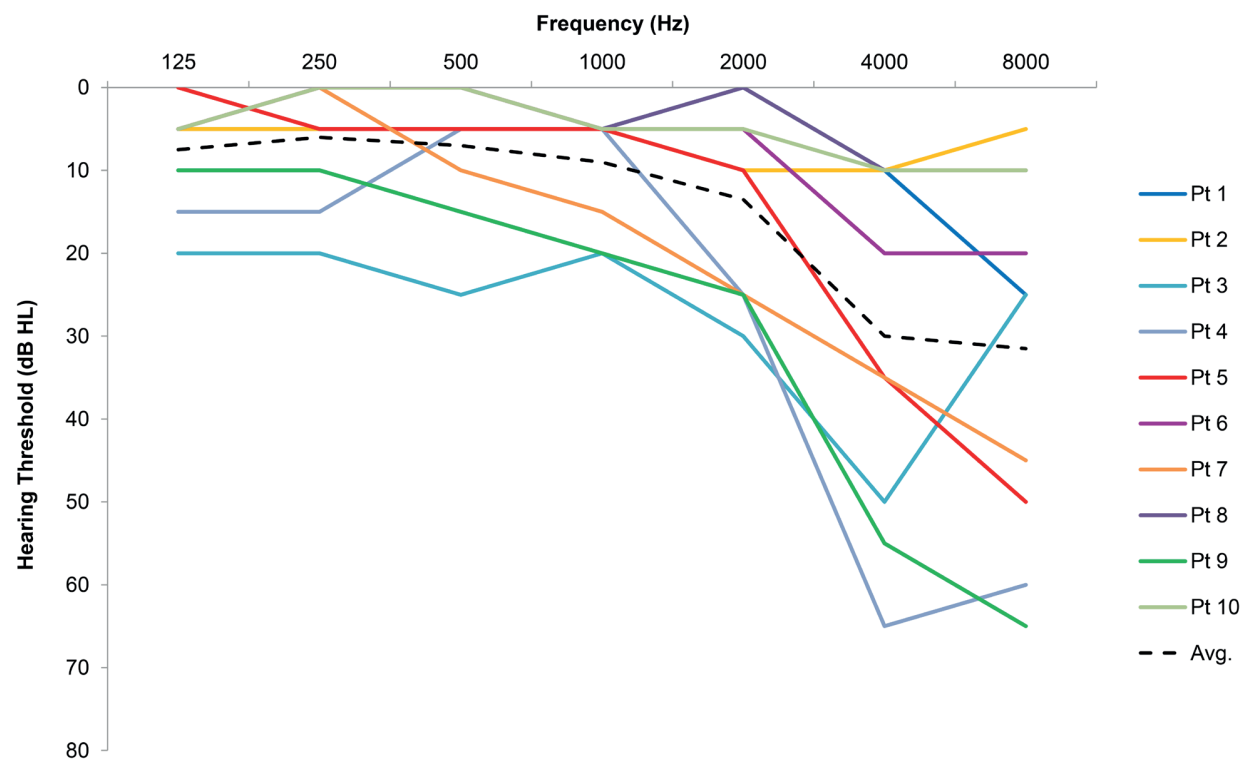




\section{Vocoder}

To evaluate the similarity of CI simulations to actual CI hearing, we created stimuli using three vocoders, termed A, B and C. Vocoder A has been developed for experiments similar to ours by the CI lab of the Arizona State University. Vocoder B was originally designed by various collaborating psychoacoustic research groups with the goal to study CI simulations in NH subjects. ${ }^{16}$ Lastly, vocoder C was developed by Cochlear Ltd.

Vocoder A and B share the same principles. First, signals are filtered with a bank of Butterworth filters into a predefined number of frequency bands (channels). The cut-off frequencies can be set to mimic the map of a CI (based on Greenwood ${ }^{17}$ distribution). Second, using half-wave (vocoder A) or full-wave (vocoder B) rectification, usually with a low-pass filter, the envelope of every band is calculated. Third, the carrier is modulated with the band envelope for every band. In our experiment, we used the two most common carriers: a noise carrier and a sine carrier. Finally, by adding all modulated carriers and by normalizing to the loudness of the original signal, a vocoded signal of the input is created. Vocoder $\mathrm{C}$ uses a Discrete Fourier Transformation-based filter bank. The envelope is captured using the quadrature envelope detection method. ${ }^{18}$ The settings of the most often adjusted vocoder parameters are explained in Supplemental Digital Content 1.

For vocoder A, we used 20 channels (maximum for this vocoder), for vocoder B and C we used 22 channels (like in Cochlear CIs).

\section{Stimuli}

We applied the vocoder simulations to six different stimuli: four speech stimuli and two music stimuli.

\section{$\underline{3.1 \text { Speech }}$}

Intelligible speech:

1. a Dutch sentence ("de bal vloog over de schutting", duration 2.46 seconds) spoken by a female speaker ${ }^{19}$ (“Female_NL”); 
2. a Dutch sentence ("de jongen kreeg een nieuwe voetbal", duration 2.29 seconds) spoken by a male speaker ${ }^{20}$ (“Male_NL”);

3. an English sentence ("I ate a salad for lunch", duration 1.66 seconds) spoken by a female speaker from the AzBio sentences ${ }^{21}$ ("Female_Eng”);

All patients were native Dutch speakers. All patients were taught English in high school, and they all considered English their second language.

Unintelligible speech:

4. a fragment of the International Speech Test Signal ("ISTS") 22 (duration 5.00 seconds). This signal is comprised of short fragments of six languages (English, Chinese, Spanish, Arabic, French, German) and has the spectrum of average speech. The advantage of using this stimulus is that patients are not distracted by an effort to understand the sentence.

\subsection{Music}

5. Acoustic guitar music (“Guitar”, duration 12.93 seconds; Passenger - Let her go);

6. Piano music ("Piano", duration 10.16 seconds; J.S. Bach - Aria of Goldberg variations).

We enclosed 17 examples of audio files, including vocoded simulations as well as original stimuli for comparison, as Supplemental Digital Content 2. 


\section{Test set-up}

All experiments were performed in a soundproof booth. Stimuli were presented from a Yamaha loudspeaker at $110 \mathrm{~cm}$ height (head level in seated position) and $130 \mathrm{~cm}$ in front of the patient. All original stimuli and the vocoded simulations were scaled at a presentation level of $65 \mathrm{~dB}(\mathrm{~A})$. If needed, the original stimulus presented to the $\mathrm{CI}$ was adjusted to match the loudness of the vocoded sound presented to the non-implanted ear. Experiments lasted for approximately 2 hours per patient.

\section{$\underline{4.1 \text { Per stimulus }}$}

\section{Phase I}

During phase I, patients listened to the original sound with their CI ear only (Figure 2a). We considered two options to listen with the CI ear only: 1) to mask the non-implanted ear with noise, identical to the masking used in the rehabilitation phase, or 2) via Direct Acoustic Input (DAI). Both options have advantages and disadvantages. In this study, we opted for the first option because the signal processing via DAI is different when compared to input via the microphone. Moreover, our patients never listen via DAI in daily life, whereas they were familiar with the masking of the non-implanted ear with the speech-shaped noise via the insert earphone. Finally, the acoustics of the room were now also taken into account.

In the case of speech stimuli, we asked patients to repeat the sentence and to identify the gender of the speaker. In the case of music stimuli, we asked if they could identify the musical instrument and we did not restrict their choice to piano or guitar (i.e. open question). Only after completion of Phase II per stimulus, we told the patient if their answers were correct or not. Each stimulus was repeated 3 to 5 times to allow the patient to obtain a solid impression of the sound, so they were able to make a good comparison in Phase II. 
Figure 2. Test set-up experiment
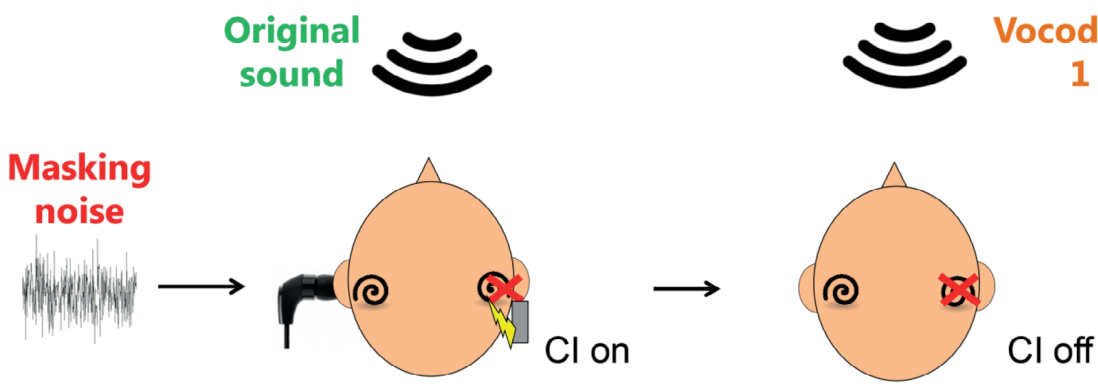

A

Phase I

B Phase II

Example: Female_Eng
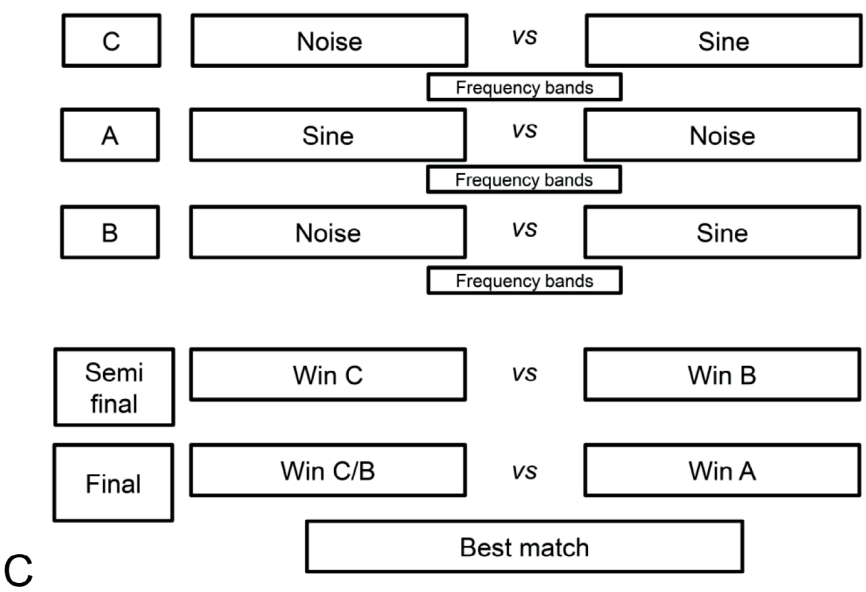

Legend:

A) Phase I: Patients listened with the CI ear only (non-implanted ear masked) to the original sound presented from a loudspeaker positioned in front of the patient.

B) Phase II: Patients listened with their non-implanted ear only (CI off) to two simulations presented from the same loudspeaker positioned in front of the patient. Patients were asked to choose which of the two simulations had greater similarity to the sound as perceived by the CI ear during Phase I.

C) Example of the outline of the test procedure for stimulus Female_Eng. For an explanation of the test procedure, see text. 


\section{Phase II}

During phase II, patients took off their CI processor and listened with only their nonimplanted ear to two vocoded stimuli (Figure 2b). Patients had to choose which of the two vocoded stimuli had greatest similarity to the original sound as perceived by their CI ear in Phase I.

The order of presentation of the vocoders (A, B, C) and carriers (noise, sine) was randomized. An example of the whole experiment procedure is shown in Figure 2c. In this example, the stimulus was Female_Eng and the carriers were compared for vocoder C (noise $v s$ sine). We recorded the patient's preference for the first or second simulation. The two first-tested stimuli always had maximum frequency bandwidth (Low-High). In order to find greater similarity, we applied low pass $(250 \mathrm{~Hz}-\mathrm{High}, 500 \mathrm{~Hz}-\mathrm{High})$ or high pass (Low-4000 $\mathrm{Hz}$, Low-2000 Hz) filtering to the selected stimulus. Due to limited time, we were not able to test all frequency bands for all speech stimuli in all patients (for details see Supplemental

Digital Content 3). The finally preferred simulation was the "winner" of the first comparison.

Subsequently, in this example, we tested vocoder A (sine vs noise) and B (noise vs sine). From these three comparisons, the "winning" vocoded simulations per vocoder were then compared in the "semi-final" and subsequently in the "final" (Figure 2c). The vocoded simulation with greatest similarity in the final was designated as the "best match" for that stimulus.

Finally, we asked the patient to give a grade for similarity between this best match and the sound as perceived by the CI ear on a scale 1-10 (1: not similar at all, 6: similarity was fairly good, 10: completely similar). All patients were familiar with this grading system, as it is the grading system for education in The Netherlands (with $\geq 6$ as pass, and $<6$ as fail).

The procedure consisting of the two phases was performed for six stimuli. We first tested the three intelligible speech stimuli, then the unintelligible speech stimulus, and finally the two music stimuli. We randomized the order of the intelligible speech stimuli and the order of music stimuli. During the experiment, patients could always return to Phase I to 
hear the original stimulus through their CI again. There were a maximum of five consecutive comparisons between two vocoded simulations; then we presented the original stimulus to the CI ear again. The time for switching between Phase I and Phase II was maintained as short as possible.

\subsection{Per patient}

We examined if any links existed between patient characteristics (age, gender, side of deafness, PTA of the non-implanted ear, DoD, experience with CI, CVC phoneme score at 3 months after CI activation) and the selection of vocoders or carriers.

\section{RESULTS}

In general, patients experienced no difficulties comparing sounds presented to their nonimplanted ear with sounds presented to their CI ear.

\section{Per stimulus}

\section{Phase I}

Patients were generally able to repeat the complete sentence correctly for the intelligible speech stimuli, and they could also identify the gender of the speaker (Table 2). As expected, it was harder for patients to correctly repeat the English sentence ("Female_Eng"). About half of patients could identify the musical instrument correctly.

Table 2. Results of phase I.

\begin{tabular}{|l|c|c|c|c|c|c|}
\hline \multirow{2}{*}{ Task } & \multicolumn{5}{|c|}{ Stimulus } \\
\cline { 2 - 5 } & Female_NL & Male_NL & Female_Eng & ISTS & Guitar & Piano \\
\hline Repeat sentence & 8 & 8 & 5 & & \multicolumn{2}{|c}{ NA } \\
\hline Identify gender of speaker & 10 & 9 & 8 & \multirow{2}{*}{ NA } & & 5 \\
\hline Identify musical instrument & \multicolumn{7}{|c|}{ NA } & & 4 & 5 \\
\hline
\end{tabular}

Legend:

Number of patients (total: $\mathrm{n}=10$ ) that correctly repeated sentences, correctly identified the gender of the speakers, and correctly identified the musical instrument. NA = not applicable. 


\section{Phase II}

Figure 3a shows the patients' selection of vocoders that had the best match per stimulus. Vocoder A was selected most often. The selection for best-matched vocoder differed among stimuli. For instance, vocoder $\mathrm{C}$ was least selected as best match for speech stimuli and piano music, whereas it was most often selected for guitar music. Overall, noise was the most often selected carrier for 5 of 6 stimuli (Figure 3b). Only for stimulus guitar music, carriers noise and sine were selected equally often. We observed a great variability in the selection for vocoders and carriers, both within and between patients. For example, patient 7 had two best matches for vocoder A, two for vocoder B and two for vocoder $\mathrm{C}$, and he selected the noise and sine carrier three times each.

Figure 3. Selection of vocoders and carriers
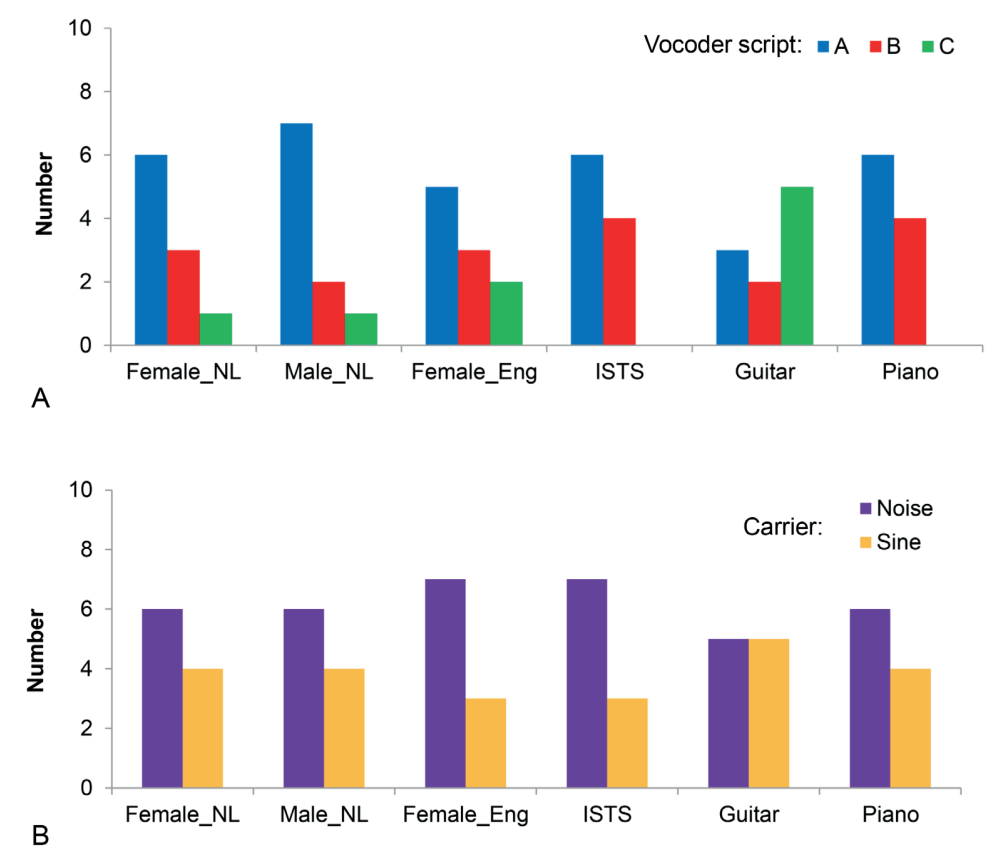

\section{Legend:}
A) Number of patients selecting vocoder A, B or C per stimulus for greater similarity with the sound as perceived by CI ear.
B) Number of patients selecting noise or sine carrier per stimulus for greater similarity with the sound as perceived by CI ear. 
The grades for similarity per stimulus are depicted in Figure 4. The average grades for similarity for both speech (6.8, average range 3.3) and music (6.3, average range 6.3) simulations were fairly good. Remarkably, the results for the ISTS stimulus were very similar to the three intelligible speech stimuli. Patients described the CI sound as "tinny", "shrill", "robotic", "electric", "scraping", or "echo-like", but they found it hard to explain what should change in our simulations to further improve the similarity to the sound as perceived by their CI ear.

Figure 4. Grades for similarity of best matches

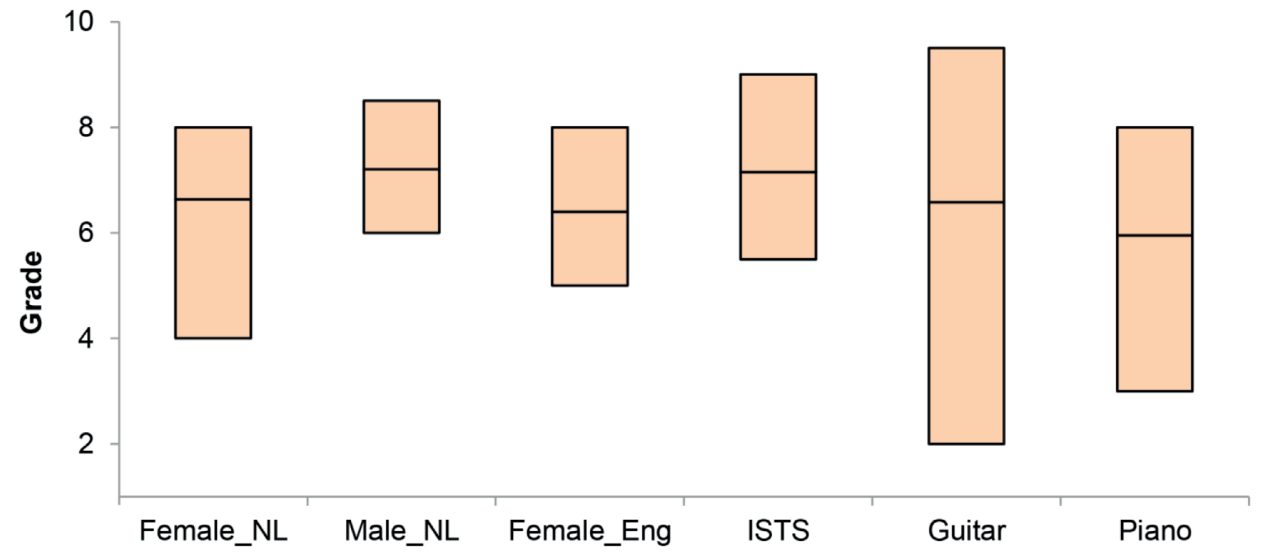

Legend: The grades for similarity of the best matches per stimulus. The horizontal line in the middle of the boxplot represents the average grade for similarity. The top and bottom of the boxes represent maximum and minimum grades for similarity.

\section{$\underline{\text { Per patient }}$}

In sum for all stimuli, 7 of the 10 patients selected vocoder $A$ as best match, followed by vocoder B (3 patients). Six patients selected noise carrier and 4 patients selected sine carrier as best match. The combination of vocoder A and carrier noise was most often selected. If multiple vocoders or carriers were selected equally often, the vocoder or carrier that received the highest grade for similarity was selected as optimal vocoder or carrier. 
We observed no clear relation between the preferred vocoders or carrier and any of the tested patient characteristics (for all patient characteristics versus vocoder and carrier: Mann-Whitney $U$ test, $p>0.05$ ). For details of the analysis of the frequency bandwidths, see Supplemental Digital Content 3.

\section{DISCUSSION}

The present study aimed to find out what a CI sounds like in a population of patients with SSD and a CI. Based on the patients' grades for similarity for our vocoded sound simulations (average 6.8 out of 10 for speech, 6.3 for music), we have a fairly good impression of the sound of a CI.

\section{Interpretation of the results}

We observed considerable variability within and between patients in their selection of vocoders and carriers. For almost all stimuli, vocoder A, B and C and both carriers noise and sine were selected by at least one patient. We found that vocoder $\mathrm{C}$ was hardly ever selected. This is a remarkable finding, as the signal processing strategy of vocoder $\mathrm{C}$ is identical to the signal processing of the processor of the CI (Cochlear Ltd.; see Supplemental Digital Content 1). Although choices for CI simulations varied greatly among patients, various patient characteristics did not seem to influence the selection for the best match of vocoder and carrier. Other patient characteristics may have been of influence, such as electrode insertion depth, cochlear size, variations in survival of neural tissue or etiology. ${ }^{13}$ However, we did not evaluate these patient characteristics in the present study.

It is important to consider the disadvantages associated with both noise and sine vocoders. The selection for a certain carrier might be stimulus-dependent. In the comparisons with music stimuli, the patient relies on temporal envelope cues of the stimuli. We therefore expected the sine carrier to be selected more often than the noise carrier. ${ }^{23}$ In contrast, we expected the noise carrier to be selected more often for speech stimuli, because this task 
relies more on spectral information of the stimulus. Our observations roughly followed these expectations. The sine carrier was selected more often for music stimuli than for speech stimuli and the noise carrier was selected more often for speech stimuli. Moreover, one should keep in mind that the output of a vocoder is acoustic, which is then subjected to the peripheral processing by the non-implanted ear with a large dynamic range, while the CI involves direct electric stimulation which drastically reduces the dynamic range. ${ }^{24,25}$ The limitations of the vocoders (further discussed by Stone et al. ${ }^{25}$ ) explain that the grades for similarity did not approach a perfect score of 10 out of 10 .

Although vocoder software programs have been used to simulate the sound of a CI for more than 20 years ${ }^{3}$, it only recently became possible to validate these acoustic models by comparisons in patients with SSD and a CI. Thus far, three studies investigated the sound of a CI in this population. First, Svirsky et al. tested one SSD patient with a Cochlear CI using speech stimuli. ${ }^{13}$ They described a direct perceptual comparison of the output of an acoustic model to the stimulation provided by a CI. Like in our study, this patient also gave the highest grade for similarity to a noise carrier. However, it is unclear which vocoder settings were used in their study. Second, Lazard et al. presented simulations of the sound that five patients with asymmetric hearing loss heard when their most apical electrode was stimulated. ${ }^{26}$ It was reported to sound like an inharmonic complex. However, the hearing of the non-implanted ear was poor, and speech or music stimuli were not tested. Third, Dorman et al. tested four stimuli in eight CI patients with SSD. ${ }^{27}$ In contrast to our results, they concluded that the sound of noise and sine vocoders does not generally correspond to the sound of a CI (grades for similarity $1.9-5.5$ on a scale $0-10$ ). In a consecutive experiment in three juvenile patients using a different approach, i.e. band pass filtering and spectral smearing of the original sounds, they were able to remarkably improve the grade for similarity that the patients gave to CI simulations (grade 10 out of 10 ). The discrepancy between their maximum grades for similarity and ours may be explained by the fact that they rated all stimuli, whereas we only rated the best match.

The descriptions of the sounds of the CI and the vocoded stimuli ("tinny", "shrill", 
"robotic", "electric", "scraping", or "echo-like") were hard to translate to adjustments of the vocoder parameters. These descriptions of the sound of a CI by our SSD patients are quite similar to descriptions given by bilaterally deaf patients with a CI. Yet, it is important to note that we cannot simply generalize our findings from SSD CI patients to CI patients with other forms of hearing impairment. For example, bilaterally deaf CI patients, in particular those who acquired deafness early in life, and patients with a longer DoD than our patients may perceive the sound of their CI differently. The central auditory system of SSD patients is expected to be different than the auditory system of bilaterally deaf patients. ${ }^{28-30}$

\section{$\underline{\text { Methodological considerations }}$}

Our test set-up may unintentionally have led to testing parts of the patient's short-term memory. Patients were instructed to focus on how the original stimulus sounded through their CI (Phase I) and then compare two vocoded stimuli to the sound as perceived by the

CI ear (Phase II). They may have memorized the CI sound differently, which may have contributed to their selection of vocoder and carrier. Moreover, in the case of speech stimuli, they may have unintentionally opted for the simulation with greater intelligibility, instead of the simulation with greater similarity. Therefore, we also tested unintelligible speech (ISTS); and the results were very similar to intelligible speech.

The masking noise in the non-implanted ear during Phase I may have unknowingly led to distraction, or might even have caused patients to judge that the stimulus with carrier noise had greatest similarity. On the other hand, an advantage of our set-up is that the route of the sound is now identical to the route of normal sounds (in contrast to DAI). Moreover, our SSD patients are familiar with the masking noise in their non-implanted ear.

We did not systematically assess the preference for the number of channels of the vocoders in all patients. After a pilot session with a few patients, these patients indicated that the maximum number of channels (i.e. 20 or 22 instead of 4-8) clearly gave the greatest similarity to the sound as perceived by the CI ear. The difference between 20 and 22 channels 
alone is unlikely to cause differences in the judgment of the CI patients, since CI users seem unable to make use of all separate channels with 10 channels or more. ${ }^{4,31,32}$ Therefore, we discarded the tests with fewer channels in an early stage of the experiments.

Test sessions already lasted two hours in our current experimental set-up, and adding more variables could have led to undesirably long test durations and decreased patient attention.

\section{Future directions}

In the present study, we performed a cross-sectional analysis investigating our CI patients at one moment in time. Based on conversations with implanted patients, their best match is likely to change over time; patients with a longer experience with their CI reported that a "less heavily modified sound" was more similar to the sound as perceived by their CI ear. This finding might suggest that the brain adapts to the new form of input based on neural plasticity. ${ }^{1}$ Furthermore, it would be interesting to also compare the sound of a CI using the DAI, because then patients can switch between the original stimulus presented to their nonimplanted ear and the simulation presented to their CI ear faster. Finally, future research could focus on reaching a closer match to the sound as perceived by the CI ear by using several new sound modifications (e.g. delay or echo) to modify the sound after or instead of vocoder modifications.

\section{CONCLUSION}

The sound of a CI seems to vary between patients. Yet, based on the fairly high similarity indicated by patients, we have a fairly good idea how a CI can sound. We can use this knowledge to inform and educate CI candidates, patients and their family members about the sound of a CI. Importantly, our results should be interpreted cautiously and cannot simply be generalized to CI patients with other forms of hearing impairment. 


\section{SUPPLEMENTAL DIGITAL CONTENT}

The Supplemental Digital Content can be accessed via https://journals.lww.com/otologyneurotology/Fulltext/2018/07000/The Sound of a Cochlear Implant Investigated in.9.aspx

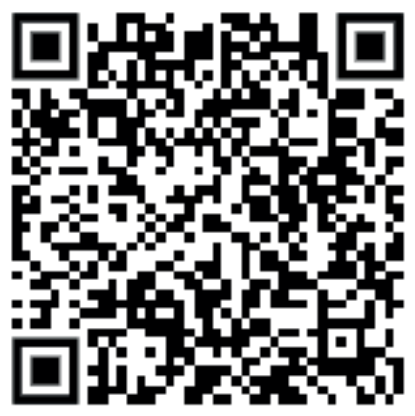

SUPPLEMENTAL DIGITAL CONTENT 1: Vocoder settings

SUPPLEMENTAL DIGITAL CONTENT 2: ZIP-file containing audio files

SUPPLEMENTAL DIGITAL CONTENT 3: Frequency bandwidths

\section{Acknowledgement}

We would like to thank the authors of vocoders A (M. Dorman and S. Natale, CI Lab of the Arizona State University, Tempe, AZ), B (via E. Gaudrain and D. Baskent, University Medical Center Groningen, Groningen, The Netherlands), and C (F. Vanpoucke and O. Qazi, Cochlear Ltd.) for use of their MATLAB vocoders.

\section{Funding}

This study was partly funded by Cochlear Ltd. as an unrestricted research grant. By research contract, Cochlear Ltd. did not have influence on the study design, data collection, analysis, data interpretation, and publication. 


\section{REFERENCES}

1. Moore DR, Shannon RV. Beyond cochlear implants: awakening the deafened brain. Nat Neurosci 2009;12(6):686-691.

2. Kohlberg G, Spitzer JB, Mancuso D, Lalwani AK. Does cochlear implantation restore music appreciation? Laryngoscope 2014;124:587-588.

3. Shannon RV, Zeng FG, Kamath V, Wygonski J, Ekelid M. Speech recognition with primarily temporal cues. Science 1995;270(5234):303-304.

4. Bingabr M, Espinoza-Varas B, Loizou PC. Simulating the effect of spread of excitation in cochlear implants. Hear Res 2008;241(1-2):73-79.

5. Dorman MF, Loizou PC. Speech intelligibility as a function of the number of channels of stimulation for normal-hearing listeners and patients with cochlear implants. Am J Otol 1997;18(6 Suppl):S113-114.

6. Bernstein JG, Iyer N, Brungart DS. Release from informational masking in a monaural competing-speech task with vocoded copies of the maskers presented contralaterally. J Acoust Soc Am 2015;137(2):702-713.

7. Churchill TH, Kan A, Goupell MJ, Ihlefeld A, Litovsky RY. Speech perception in noise with a harmonic complex excited vocoder. J Assoc Res Otolaryngol 2014;15(2):265-278.

8. Ma N, Morris S, Kitterick PT. Benefits to Speech Perception in Noise From the Binaural Integration of Electric and Acoustic Signals in Simulated Unilateral Deafness. Ear Hear 2016; 37(3):248-259.

9. Jones H, Kan A, Litovsky RY. Comparing Sound Localization Deficits in Bilateral Cochlear-Implant Users and Vocoder Simulations With Normal-Hearing Listeners. Trends Hear 2014;18:2331216514554574.

10. Schvartz KC, Chatterjee M. Gender identification in younger and older adults: use of spectral and temporal cues in noise-vocoded speech. Ear Hear 2012;33(3):411-420.

11. Gaudrain E, Başkent D. Factors limiting vocal-tract length discrimination in cochlear implant simulations. J Acoust Soc Am 2015;137(3):1298-1308. 
12. Fuller CD, Gaudrain E, Clarke JN, Galvin JJ, Fu QJ, Free RH, Başkent D. Gender categorization is abnormal in cochlear implant users. J Assoc Res Otolaryngol 2014;15(6):1037-1048.

13. Svirsky MA, Ding N, Sagi E, Tan CT, Fitzgerald M, Glassman EK, Seward K, Neuman AC. Validation of acoustic models of auditory neural prostheses. Proc IEEE Int Conf Acoust Speech Signal Process 2013;8629-8633.

14. Peters JPM, Van Zon A, Smit AL, Van Zanten GA, de Wit GA, Stegeman I, Grolman W. CINGLE-trial: Cochlear Implantation for siNGLE-sided deafness, a Randomized Controlled Trial and economic evaluation. BMC Ear, Nose and Throat Disorders 2015;15:3.

15. Bosman AJ, Smoorenburg GF. Intelligibility of Dutch CVC syllables and sentences for listeners with normal hearing and with three types of hearing impairment. Audiology $1995 ; 34(5): 260-284$.

16. Gaudrain E, Carlyon RP. Using Zebra-speech to study sequential and simultaneous speech segregation in a cochlear-implant simulation. J Acoust Soc Am 2013;133(1):502518.

17. Greenwood DD. A cochlear frequency-position function for several species--29 years later. J Acoust Soc Am 1990;87(6):2592-2605.

18. Swanson, B.A. Pitch perception with cochlear implants (PhD dissertation; 2008). Faculty of Medicine, The University of Melbourne.

19. Plomp R, Mimpen AM. Improving the reliability of testing the speech reception threshold for sentences. Audiology 1979;18(1):43-52.

20. Smoorenburg GF. Speech reception in quiet and in noisy conditions by individuals with noise-induced hearing loss in relation to their tone audiogram. J Acoust Soc Am 1992;91(1):421-437.

21. Spahr AJ, Dorman MF, Litvak LM, Van Wie S, Gifford RH, Loizou PC, Loiselle LM, Oakes T, Cook S. Development and validation of the AzBio sentence lists. Ear Hear 
2012;33(1):112-117.

22. Holube I, Fredelake S, Vlaming M, Kollmeier B. Development and analysis of an International Speech Test Signal (ISTS). Int J Audiol 2010;49(12):891-903.

23. Spahr AJ, Litvak LM, Dorman MF, Bohanan AR, Mishra LN. Simulating the effects of spread of electric excitation on musical tuning and melody identification with a cochlear implant. J Speech Lang Hear Res 2008;51(6):1599-1606.

24. Gaudrain E, Grimault N, Healy EW, Béra JC. Streaming of vowel sequences based on fundamental frequency in a cochlear-implant simulation. J Acoust Soc Am 2008;124(5):3076-3087.

25. Stone MA, Füllgrabe C, Moore BC. Benefit of high-rate envelope cues in vocoder processing: effect of number of channels and spectral region. J Acoust Soc Am 2008;124(4):2272-2282.

26. Lazard DS, Marozeau J, McDermott HJ. The sound sensation of apical electric stimulation in cochlear implant recipients with contralateral residual hearing. PLoS One 2012;7(6):e38687.

27. Dorman MF, Natale SC, Butts AM, Zeitler DM, Carlson ML. The Sound Quality of Cochlear Implants: Studies With Single-sided Deaf Patients. Otol Neurotol 2017;38(8):e268-e273.

28. Mossop JE, Wilson MJ, Caspary DM, Moore DR. Down-regulation of inhibition following unilateral deafening. Hear Res 2012;147:183-187.

29. Bilecen D, Seifritz E, Radü EW, Schmid N, Wetzel S, Probst R, Scheffler K. Cortical reorganization after acute unilateral hearing loss traced by fMRI. Neurology 2000;54:765767.

30. Kral A, Heid S, Hubka P, Tillein J. Unilateral hearing during development: hemispheric specificity in plastic reorganizations. Front Syst Neurosci 2013;7:93.

31. Friesen LM, Shannon RV, Baskent D, Wang X. Speech recognition in noise as a function of the number of spectral channels: comparison of acoustic hearing and cochlear 
implants. J Acoust Soc Am 2001;110(2):1150-1163.

32. Fishman KE, Shannon RV, Slattery WH. Speech recognition as a function of the number of electrodes used in the SPEAK cochlear implant speech processor. J Speech Lang Hear Res 1997;40(5):1201-1215. 


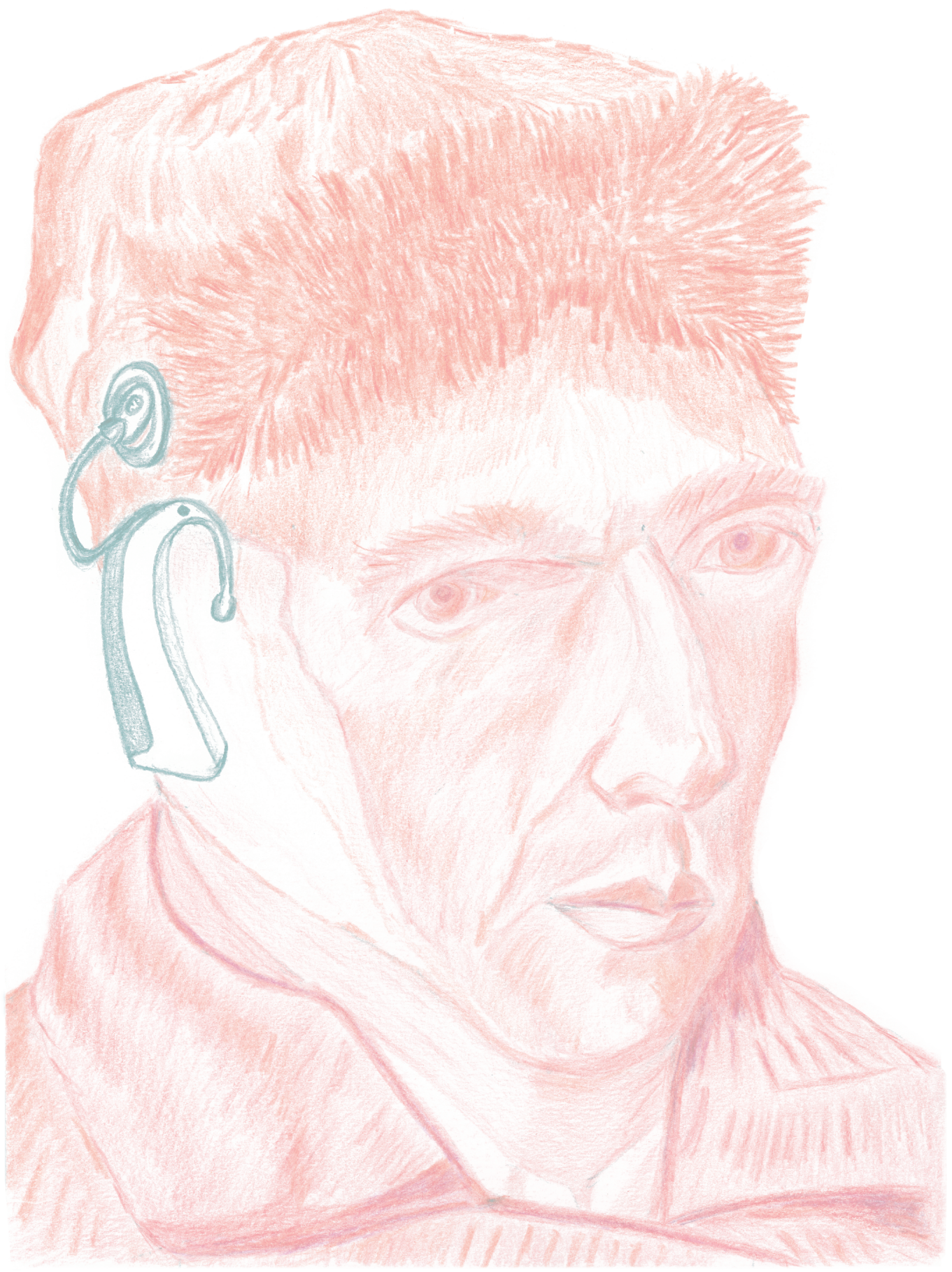




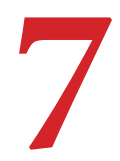

\section{Electro-acoustic Pitch Matching Experiments in Patients with Single-Sided Deafness and a Cochlear Implant}

Is there a need for adjustment of the default frequency allocation tables? 


\section{ABSTRACT}

Patients with single-sided deafness (SSD) and a cochlear implant (CI) can compare the pitch of stimulated electrodes and acoustic tones. A pitch mismatch may negatively bear on the fusion of the signals from the two ears, which may limit auditory performance. We aimed to explore this mismatch, to correlate it to performance, and finally to discuss its possible clinical consequences.

Ten patients with SSD and a CI (Cochlear Ltd.) compared the pitch of electrical and acoustic stimuli. Patients had to choose one of two acoustic stimuli, with the pitch closest to the pitch of the electrical stimulus at electrodes $3,7,11,15$, and 19. The difference between the two acoustic stimuli iteratively decreased from 2 octaves to $1 / 8$ octave, resulting in a "pitch match" per electrode. Furthermore, we computed the insertion angle of the CI electrode array based on high-resolution computed tomography scans. Subsequently, we created frequencyplace maps. The difference between our pitch matches and two references (the spiral ganglion map and the default frequency allocation by Cochlear Ltd.) was defined as "mismatch".

We observed large intra- and intersubject variability. Following the tonotopic organization of the cochlea, we observed that the pitch matches decreased with increasing insertion angle. The pitch-matched frequencies were on average 2.0 and 1.3 octaves lower than the spiral ganglion map and the default frequency allocation, respectively. There was no significant correlation between performance (consonant-vowel-consonant phoneme recognition score) and mismatch $\left(R^{2}=0.06, P>0.1\right)$.

Given the methodological considerations, and the insignificant correlation between mismatch and performance, pitch matching results must not necessarily lead to a change in clinical fitting strategies. 


\section{INTRODUCTION}

Cochlear implantation partially restores auditory input to the patient deafened by profound damage of the cochlea. In the Netherlands, bilaterally deaf patients receive one cochlear implant (CI), and can regain speech perception after a rehabilitation phase. A CI has an electrode array of 12 to 22 electrodes (depending on the manufacturer of the CI), which is surgically implanted in the cochlea to distribute auditory information; each electrode supposedly stimulates different auditory nerve fibers, enabling a different perceived pitch. In current methods of the coding of sound to CI electrodes, adjacent acoustic frequency bands are assigned to adjacent electrodes: the lowest frequencies to the deepest inserted electrode (for all brands a center frequency of about $200 \mathrm{~Hz}$ ), the highest frequencies to the shallowest inserted electrode $(8,000 \mathrm{~Hz})$. This design mimics the tonotopic organization of the normal cochlea.

Recently, patients with single-sided deafness (SSD) or asymmetrical hearing loss have been implanted with a CI. These patients must fuse the electrical input from their CI with the acoustic input from their better ear. Consequently, these patients can compare the pitch of a stimulated electrode in their CI ear to the pitch of acoustic tones perceived by their normal hearing ear. A possible mismatch in pitch between ears may have detrimental effects on the fusion of the signals from the two ears, which may limit performance. ${ }^{1,2}$ Therefore, it is important to deliver auditory information at the correct cochlear location. ${ }^{3}$

The Greenwood function describes the normally transduced frequency by the organ of Corti at a given place in the cochlea. ${ }^{4}$ However, the Greenwood function may not be suitable for CI patients, since the electrode array of a CI stimulates the spiral ganglion cells and not the organ of Corti. ${ }^{5,6}$ Therefore, Stakhovskaya et al. performed a histological analysis to construct a spiral ganglion cell map of the cochlea, which is theoretically more suitable for describing the frequency-place relation in CI patients..$^{5,7,8}$

In order to construct frequency-place maps, it is important to correlate the matched pitch to the intracochlear position of the electrode contacts. The intracochlear electrode 
insertion is preferably expressed as insertion angle (degrees, ${ }^{\circ}$ ) instead of insertion depth $(\mathrm{mm})^{6,7}$, because the size of the cochlea may differ amongst patients $\mathrm{s}^{7,9,10}$ and the intracochlear position of the electrode array (e.g. perimodiolar versus near the lateral wall) may lead to different insertion angles for identical insertion depths.

Several studies ${ }^{5,6,11,12}$ investigating pitch matching in patients with SSD found a mismatch of 1 to 2 octaves compared to the values as predicted by Stakhovskaya et al. However, these studies also included patients with moderate to severe hearing loss in the better ear. Their hearing loss may have a negative impact on the ability to make reliable pitch comparisons, because cochlear hearing loss causes distorted pitch perception. ${ }^{13}$ Furthermore, earlier studies used X-rays with a modified Stenvers view ${ }^{14}$ to image the electrode position..$^{2,5,6,11,12,15,16}$ The use of cochlear X-ray imaging for intersubject comparison is prone to error because the angle of X-ray is seldom identical between patients and the method leaves room for inter-observer error. Nowadays, high-resolution computed tomography (HRCT) images can be obtained to image the intracochlear electrode position with greater precision and less intersubject variability. ${ }^{17}$

In the present study, ten SSD patients implanted with a CI compared the pitch of an electrical stimulus presented to the CI ear to the pitch of acoustic stimuli presented to the better ear. All patients had good hearing in their better ear. Subsequently, we objectified the intracochlear electrode position using HRCT images. The resulting frequency-place map provides information about a possible mismatch between the electrically and acoustically stimulated ear. Our aim was to explore this possible mismatch, and see if it was correlated with performance on a phoneme recognition task. Finally, we discussed its possible clinical consequences. 


\section{METHODS}

\section{Patients}

Ten patients with SSD and a CI participated in our experiment. All patients received a CI as participants in a current Randomized Controlled Trial evaluating three treatment modalities for SSD: contralateral routing of sound systems and bone conduction devices are compared to cochlear implantation. ${ }^{18}$ This trial is ethically approved by the Institutional Review Board of the University Medical Center Utrecht (NL45288.041.13) and all participants provided written Informed Consent prior to trial participation. Table 1 shows all individual patient characteristics. The patients used their CI on average for $9.2 \pm 4.5$ hours per day at the time of their experiment.

Table 1. Patient characteristics of the included patients

\begin{tabular}{|c|c|c|c|c|c|c|c|c|}
\hline Patient & Gender & $\begin{array}{l}\text { Age at } \\
\text { experiment } \\
\text { (years) }\end{array}$ & $\begin{array}{l}\text { Deaf } \\
\text { Ear }\end{array}$ & Etiology & $\begin{array}{l}\text { Duration } \\
\text { of deafness } \\
\text { (years) }\end{array}$ & $\begin{array}{l}\text { Experience } \\
\text { CI (months) }\end{array}$ & $\begin{array}{l}\text { PTA } \\
\text { better ear } \\
(\mathrm{dB} \mathrm{HL}) \\
\end{array}$ & $\begin{array}{l}\text { CVC } \\
\text { phoneme } \\
\text { score }\end{array}$ \\
\hline Pt 1 & Female & 57.0 & Left & Iatrogenic & 8.7 & 16.2 & 5.0 & $91 \%$ \\
\hline Pt 2 & Female & 55.7 & Left & Sudden deafness & 1.3 & 13.6 & 7.5 & $84 \%$ \\
\hline Pt 3 & Male & 68.0 & Left & Labyrinthitis & 3.3 & 6.3 & $31.3^{*}$ & $48 \%$ \\
\hline Pt 4 & Male & 61.9 & Right & Sudden deafness & 2.1 & 12.5 & 25.0 & $76 \%$ \\
\hline Pt 5 & Male & 58.8 & Right & Unknown & 9.9 & 3.4 & 13.8 & $59 \%$ \\
\hline Pt 6 & Male & 47.3 & Left & Ménière's disease & 4.7 & 3.1 & 7.5 & $83 \%$ \\
\hline Pt 7 & Male & 38.1 & Right & Unknown & 3.2 & 6.5 & 21.3 & $93 \%$ \\
\hline Pt 8 & Male & 49.4 & Right & Ménière's disease & 3.3 & 5.7 & 3.8 & $79 \%$ \\
\hline Pt 9 & Male & 64.4 & Left & Unknown & 4.2 & 3.2 & 28.8 & $94 \%$ \\
\hline Pt 10 & Female & 24.1 & Left & Sudden deafness & 1.7 & 3.4 & 5.0 & $78 \%$ \\
\hline
\end{tabular}

\section{Legend:}

* Patient 3 (Pt 3) had a PTA of the better ear of $30.0 \mathrm{~dB}$ HL at time of inclusion, but his hearing slightly deteriorated. Abbreviations: PTA = Pure Tone Average (500, 1000, 2000 and 4000 Hz), CVC = Consonant-Vowel-Consonant phoneme score at 3 months after CI activation for the CI-ear only (better ear masked with masking noise). 
To be eligible for inclusion in the trial, adult patients must have a duration of deafness (DoD) between 3 months and 10 years. Furthermore, their Pure Tone Average threshold in the better ear $\left(\mathrm{PTA}_{\mathrm{be}} ; 500,1000,2000\right.$ and $\left.4000 \mathrm{~Hz}\right)$ was at most $30 \mathrm{~dB} \mathrm{HL}$, and in the poor ear at least $70 \mathrm{~dB}$ HL. The audiograms of the better ears of the ten patients are depicted in Figure 1 (patient 3 had a PTA ${ }_{b e}$ of $30.0 \mathrm{~dB} \mathrm{HL}$ at time of inclusion, but his hearing slightly deteriorated which resulted in a $\mathrm{PTA}_{\mathrm{be}}$ of $31.3 \mathrm{~dB} \mathrm{HL}$ at the time of analysis).

Figure 1. Pure tone audiometry (dB HL) of better ears of patients.

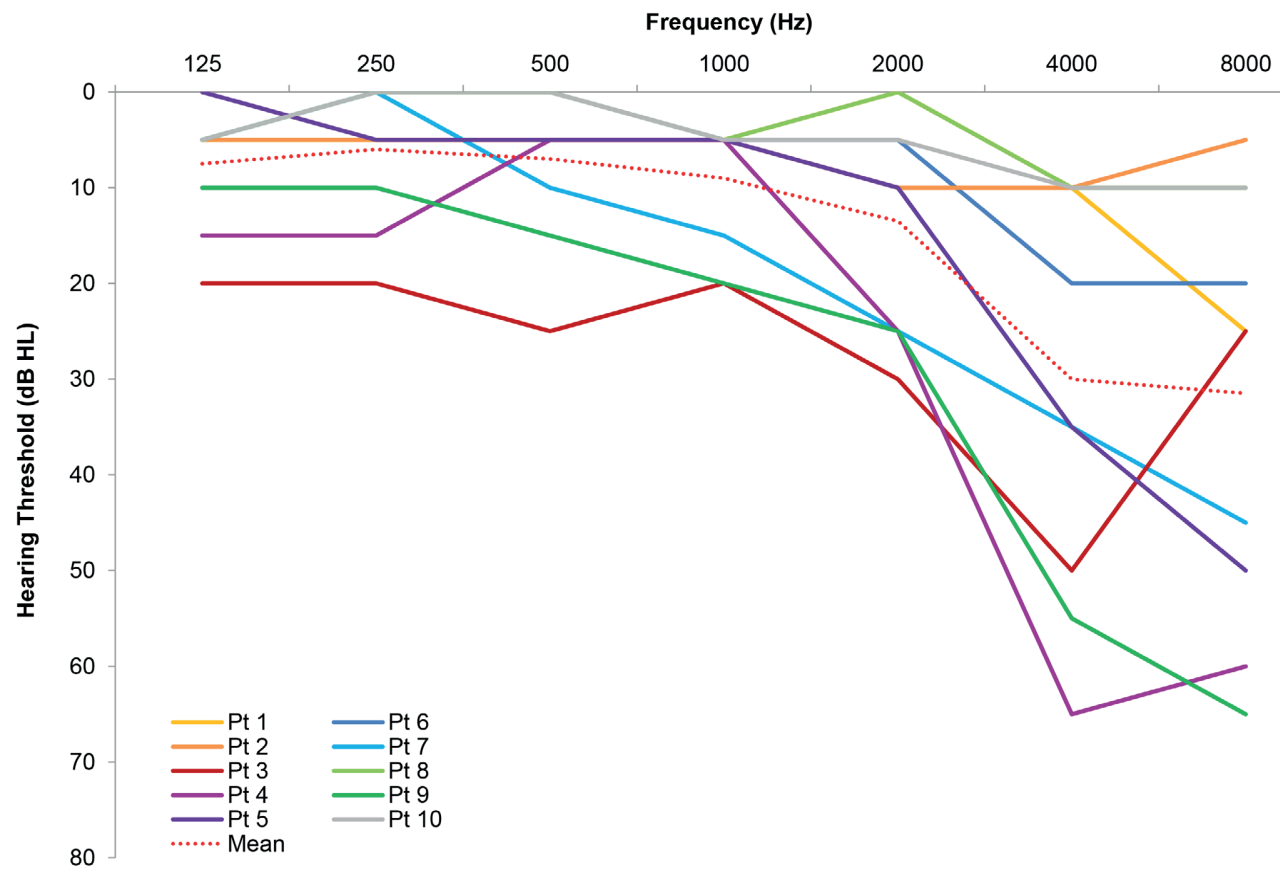




\section{Cochlear Implants}

All ten patients were implanted with a Nucleus CI (type CI 422, Cochlear Ltd.) with a Slim Straight electrode array. The Slim Straight electrode array consists of 22 electrode contacts: number 1 being the most basal electrode (high frequency tones) and number 22 being the most apical electrode (low frequency tones). The allocated center frequencies per electrode (in the default frequency allocation table of Cochlear Ltd.) are depicted in Table 2.

For two patients, electrodes were switched off due to high impedances at CI activation (patient 1: electrode 2, patient 5: electrodes 1 and 2). Consequently, the center frequencies of the remaining electrodes differed from default because of the automatic reallocation (see Supplementary Material 1 for frequency allocations per patient). These new center frequencies were used in all following measurements and calculations. The geometric mean of the center frequencies per electrode of all patients is referred to as the "Cochlear reference line" in the remainder of this paper. 


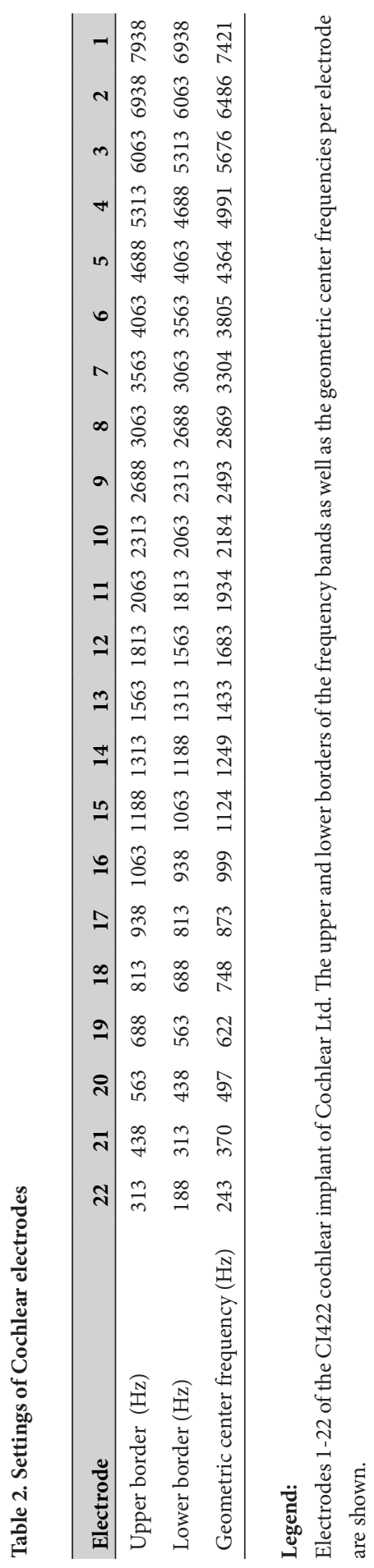




\section{Stimuli}

\section{$\underline{\text { Electric stimuli }}$}

To present the electric stimuli to the CI, we used the Custom Sound software package (version 4.3, Cochlear Ltd.). We stimulated five electrodes separately (electrodes 3, 7, 11, 15, 19; distributed along the complete electrode array from the basal to the apical region) in all patients at a comfortable level (C-level of the most frequently used program).

The order of electrode measurements was created by Latin square design (see Supplementary Material 2). There are two advantages of the order created by the Latin square over a randomized order. First, the Latin square order made sure we would not test the same electrode two times in a row. Second, all electrodes were tested four times. Randomization might have resulted in an unequal number of measurements per electrode (e.g. two measurements of electrode 3 and six measurements of electrode 19). The same order of electrodes was used for all patients. We measured all five electrodes four times (runs $1-4$ ), to collect multiple measurements per electrode.

The stimulus consisted of a continuously repeated biphasic pulse train (pulse duration $25 \mu$ s, pulse repetition rate $900 \mathrm{~Hz}$, train duration $5000 \mathrm{~ms}$ ) followed by a silent interval of $200 \mathrm{~ms}$. The silent interval provides sufficient time for nerve condition restoration.

\section{$\underline{\text { Acoustic stimuli }}$}

We used pure tones as acoustic stimuli, like Boëx et al. ${ }^{6}$ Although a pure tone is not identical to the sound of a pulse train stimulation at one specific electrode, several of our patients preferred a pure tone over $1 / 3$ noise bands or warble tones to compare it to the electric stimulus in their CI ear. The pure tones were generated by a clinical audiometer and presented from a loudspeaker at $110 \mathrm{~cm}$ height (head level in seated position) and $130 \mathrm{~cm}$ distance in front of the patient. The manually-presented acoustic stimuli had minimum durations of 500 ms.

For the first run of measurements per electrode, we adjusted the level of a $1 \mathrm{kHz}$ 
acoustic tone (median $60 \mathrm{~dB} \mathrm{HL}$, range $50-65 \mathrm{~dB} \mathrm{HL}$ ) to match it to the loudness of the electric stimulus in the $\mathrm{CI}$ ear (C-level of the most frequently used program). In this range (50 - $65 \mathrm{~dB} \mathrm{HL})$, the influence of loudness on pitch perception is negligible. ${ }^{19}$ Subsequently, the level of the acoustic tones in runs 2,3 , and 4 per electrode was identical to the level of the first run.

\section{Experimental design}

All experiments were done by one researcher (JPMP) in a soundproof booth. While listening to the electric stimulus, the patient heard two acoustic tones with a frequency interval of 2 octaves (e.g. $250-1000 \mathrm{~Hz}$ ). The patient had to indicate which of these two tones had a pitch closer to the pitch of the electric stimulus. After the choice of the patient, more comparisons were made with a frequency interval of 2 octaves (e.g. $1000-4000 \mathrm{~Hz}$ and $500-2000 \mathrm{~Hz}$ ) to check in which direction (i.e. lower or higher frequencies) the most resembling frequency was to be found. Subsequently, the frequency interval was decreased to 1 octave (e.g. 500 $1000 \mathrm{~Hz}$ ). Again, several comparisons were made to obtain a reliable idea of which tone was most similar to the electric stimulus. This method was repeated for frequency intervals of $1 / 2,1 / 4$, and $1 / 8$ octaves between the two acoustic stimuli. An example of this procedure is provided in Supplementary Material 3. In the end, the patient indicated that one of the two acoustic stimuli had a pitch as equal as possible to the stimulus in the CI ear. We calculated the geometric means of the four matched frequencies (runs $1-4$ ) per electrode per patient, referred to as "pitch match" in the remainder of this paper.

The experiment lasted approximately 2 hours per patient, and therefore we could not test all 22 electrodes.

\section{Insertion angle of $\mathrm{CI}$ electrode}

In order to take the intracochlear electrode position per patient into account, the insertion angle of the CI electrode array was measured. Postoperative HRCT scans were obtained for 
all patients. We were able to compute the intracochlear position of specific electrode contacts using an in-house developed method, implemented in MATLAB (MATLAB R2015b, MathWorks, Natick, USA).

In short, this method first traces the center-line of the electrode array in the HRCTscan, and then detects the electrode contact positions by means of correlation with a model, which is based on the known dimensions of the electrode array. A plane was aligned to the basal turn of the cochlea by fitting it through the first full $180^{\circ}$ turn, to which the HRCT scan was reformatted. After manual identification by an experienced radiologist of two reference points per HRCT scan (the top of the modiolus and the most lateral point of the horizontal semi-circular canal ${ }^{17,20}$ (Figure 2a), the intracochlear electrode contact positions were transformed to a cylindrical coordinate system, and represented by their elevation, radius, and insertion angle (Figure $\mathbf{2 b}$ ). In the current study, we made use of the insertion angles for electrodes $3,7,11,15$, and 19 only.

These insertion angles use the line from the modiolus to the horizontal semi-circular canal as 0 -degree line. ${ }^{17}$ In contrast, Stakhovskaya et al. expressed their insertion angles from the 0 -degree line between the modiolus and the round window. ${ }^{7}$ However, the round window is not easily identified anymore on postoperative scans. ${ }^{17}$ To be able to compare our insertion angles to the angles reported by Stakhovskaya et al., we therefore added 34.6 degrees ${ }^{17}$ to the Stakhovskaya values (see Supplementary Material 1).

Knowing the insertion angle per electrode for all patients, we could calculate the predicted frequency.7 This predicted frequency is referred to as the "Stakhovskaya reference line" in the remainder of this paper. 
Figure 2. Automated assessment of intracochlear electrode position
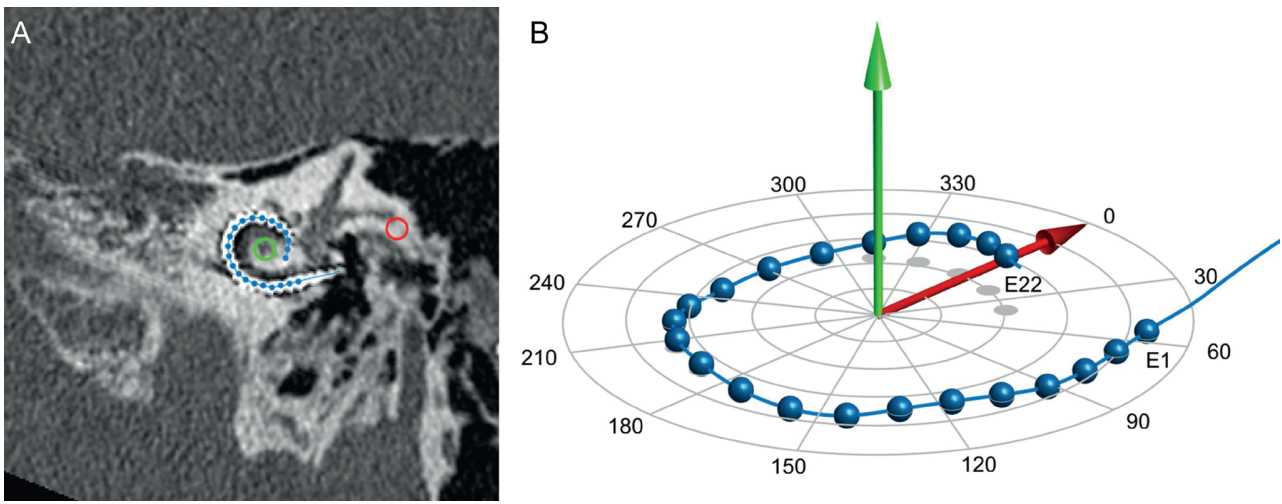

Legend:

a) Reformatted slice through the basal turn of the cochlea of patient 6 . The circles indicate the location of the two reference points: the top of the modiolus (green) and the most lateral point of the horizontal semi-circular canal (red). The 22 blue dots indicate the location of the individual electrode contacts. The distance between the center of the electrode contacts is $0.55 \mathrm{~mm}$ for electrodes $1-8,0.60 \mathrm{~mm}$ for electrodes $8-17,0.65 \mathrm{~mm}$ for electrodes $17-18$, and $0.45 \mathrm{~mm}$ for electrodes $18-22$.

b) The cylindrical coordinate system as defined by the two reference points in Figure 2a (same patient). The green arrow points towards the top of the modiolus. The red arrow points towards the most lateral point of the horizontal semi-circular canal $\left(0^{\circ}\right.$ angle). E1 = electrode 1 (most basal), E22 = electrode 22 (most apical).

\section{Data analysis}

We were interested in the mismatch between the pitch matches of our patients compared to the Stakhovskaya reference line and the Cochlear reference line. A two-sided one sample t-test was used to test if the pitch matches per electrode differed significantly from the reference lines.

We examined if there was a correlation between the mismatch and the patient characteristics listed in Table 1 (age, DoD, experience with CI, $\mathrm{PTA}_{\mathrm{be}}$ ) using linear regression. Furthermore, we tested if a correlation existed between the mismatch and the performance, using the Consonant-Vowel-Consonant (CVC) phoneme recognition scores 3 months after CI activation for the CI-ear only (better ear masked with masking noise).

Data analysis was performed using SPSS Statistics software version 21. A $P$-value of $<0.05$ was considered statistically significant. 


\section{RESULTS}

In total, we presented 2,878 comparisons of two acoustic tones to ten patients in four runs on five electrodes, resulting in a mean $( \pm \mathrm{SD})$ of $14.4 \pm 3.6$ comparisons per patient per run per electrode. We noticed that the task was often difficult for patients, which may have contributed to the observed large intra- and intersubject variability. As an example, the pitch matches of the four runs of patients 4 and 8 are plotted in Figure $\mathbf{3 a}$ and $\mathbf{3 b}$. The coordinates for the Stakhovskaya reference line differ between patients, since the coordinates are adjusted based on the insertion angle of the electrode contacts per patient. The coordinates for the Cochlear reference line are identical, since both patients used the standard frequency allocation by Cochlear (Table 2). Both within one patient between runs as well as between patients, large differences were measured. For example, the pitch matches for patient 8 for electrode 11 were $156 \mathrm{~Hz}$ in run $1,125 \mathrm{~Hz}$ in runs 2 and 3, whereas the pitch match was $2000 \mathrm{~Hz}$ in run 4 . For a closer inspection of all measurements (four runs on five electrodes in ten patients), see Supplementary Material 1.

Figure 3. Example of four runs for two patients

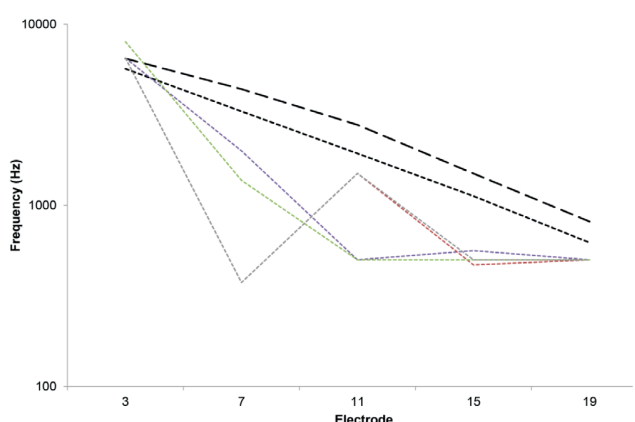

A

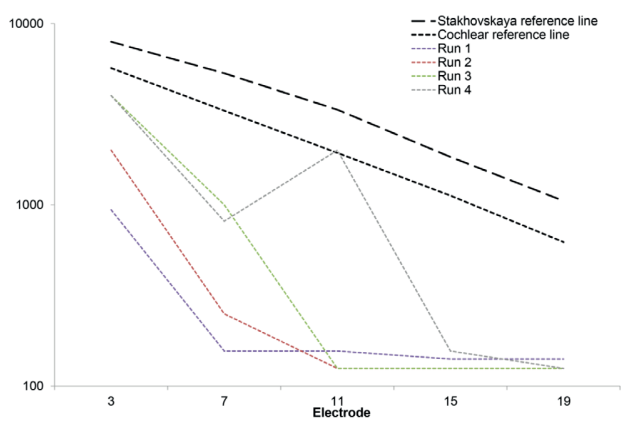

B

Legend: Examples for two patients of the four runs per electrode (panel A: patient 4, panel B: patient 8). On the horizontal axis the electrode number is depicted, the vertical axis represents frequency. As a comparison, the Stakhovskaya and Cochlear reference lines are also plotted. 
Figure 4 shows the insertion angles of all 22 electrodes for all ten patients. The insertion angle increases with increasing electrode number. The mean insertion angle of the most basal electrode (electrode 1 ) was $68^{\circ} \pm 20^{\circ}\left(\right.$ median $65^{\circ}$, range $37-102^{\circ}$ ), whereas the mean insertion angle of the most apical electrode (electrode 22 ) was $442^{\circ} \pm 55^{\circ}$ (median $450^{\circ}$, range $\left.351-537^{\circ}\right)$. The range in insertion angles was therefore larger for the most apical electrodes $\left(186^{\circ}\right)$ than for the most basal electrodes $\left(65^{\circ}\right)$. For a closer inspection of the insertion angles per electrode per patient, please see Supplementary Material 1.

Figure 4. Insertion angle per electrode number

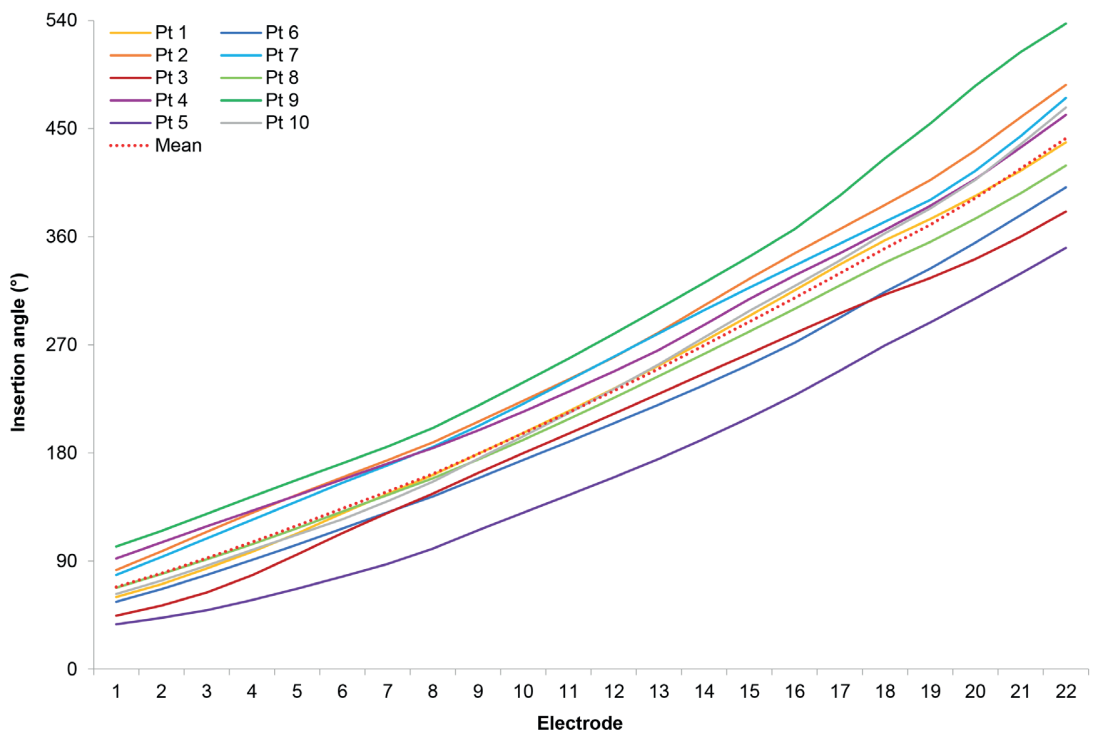

Legend: Insertion angle in degrees according to the cochlear coordinate system as defined by Verbist et al. ${ }^{17}$ The insertion angle increases with increasing electrode number (electrode 1 is the most basal electrode, electrode 22 is the most apical electrode).

In Figure 5, all pitch matches are plotted against the insertion angle. The logarithmic vertical axis shows tone frequency and also denotes musical tones (A4 $=440 \mathrm{~Hz})$. With different symbols and colors per patient, the four runs are plotted as data points per electrode against their corresponding insertion angle in degrees horizontally. The geometric means of these individual data points per electrode are shown as lines with corresponding colors. Three 
additional lines are presented: the dashed bold black line represents the Stakhovskaya reference line, the thin black dashed line represents the Cochlear reference line, and the dotted red line indicates the geometric mean of all patients' pitch matches. For the latter two lines, the insertion angle per electrode was the mean insertion angle per electrode of all patients.

Figure 5. Pitch matches per insertion angle for electrodes 3, 7, 11, 15, and 19.

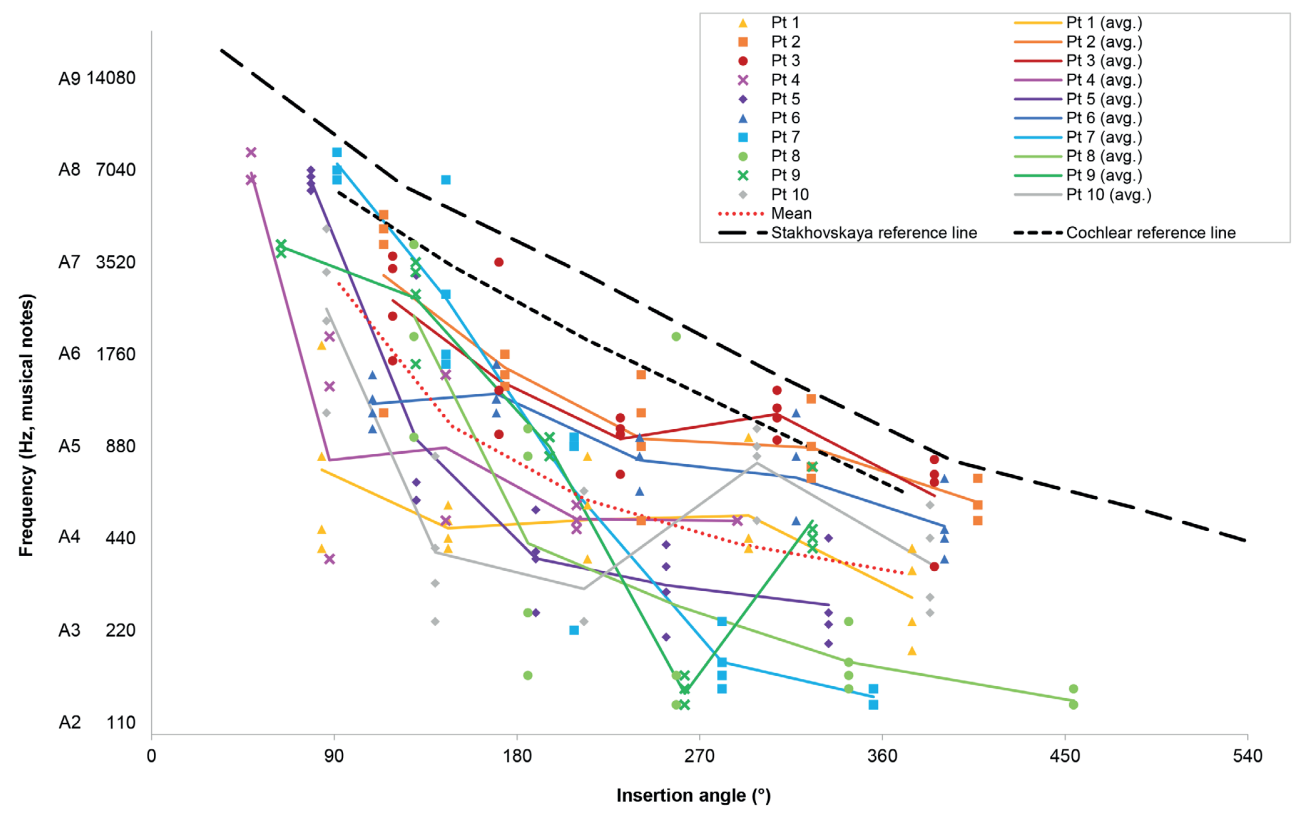

Legend: The logarithmic vertical axis shows tone frequency and also denotes musical tones. The four runs are plotted as data points per electrode $(3,7,11,15,19$; the left most data point per patient is electrode 3 , the right most data point per patient is electrode 19) against their corresponding insertion angle in degrees horizontally. When there are not four separate data points visible, some data points overlap. The dashed bold black line represents the Stakhovskaya reference line, the thin black dashed line represents the Cochlear reference line, and the dotted red line indicates the geometric mean of all patients' pitch matches.

As expected by the tonotopic organization of the cochlea and the programming of the CI, we observed that the frequency of the pitch matches decreased with increasing electrode number (i.e. towards the apex of the cochlea). However, the geometric mean of all patients' pitch matches (dotted red line) was found to be well below the Stakhovskaya and Cochlear 
reference lines.

To illustrate the mismatch between the patients' average line and the Stakhovskaya and Cochlear reference lines, in Figure 6 we expressed this difference in octaves vertically against electrode number horizontally. For all electrodes, the average mismatch is 2.0 octaves lower than the Stakhovskaya reference line, and 1.3 octaves lower than the Cochlear reference line. The mismatch is larger for electrodes in the middle area of the electrode array compared to electrodes in the apical and basal areas of the electrode array. The mismatch is significant for each of the five tested electrodes $(T(9)<-2.883, P<0.02$, two-sided).

Figure 6. Octave difference to reference lines

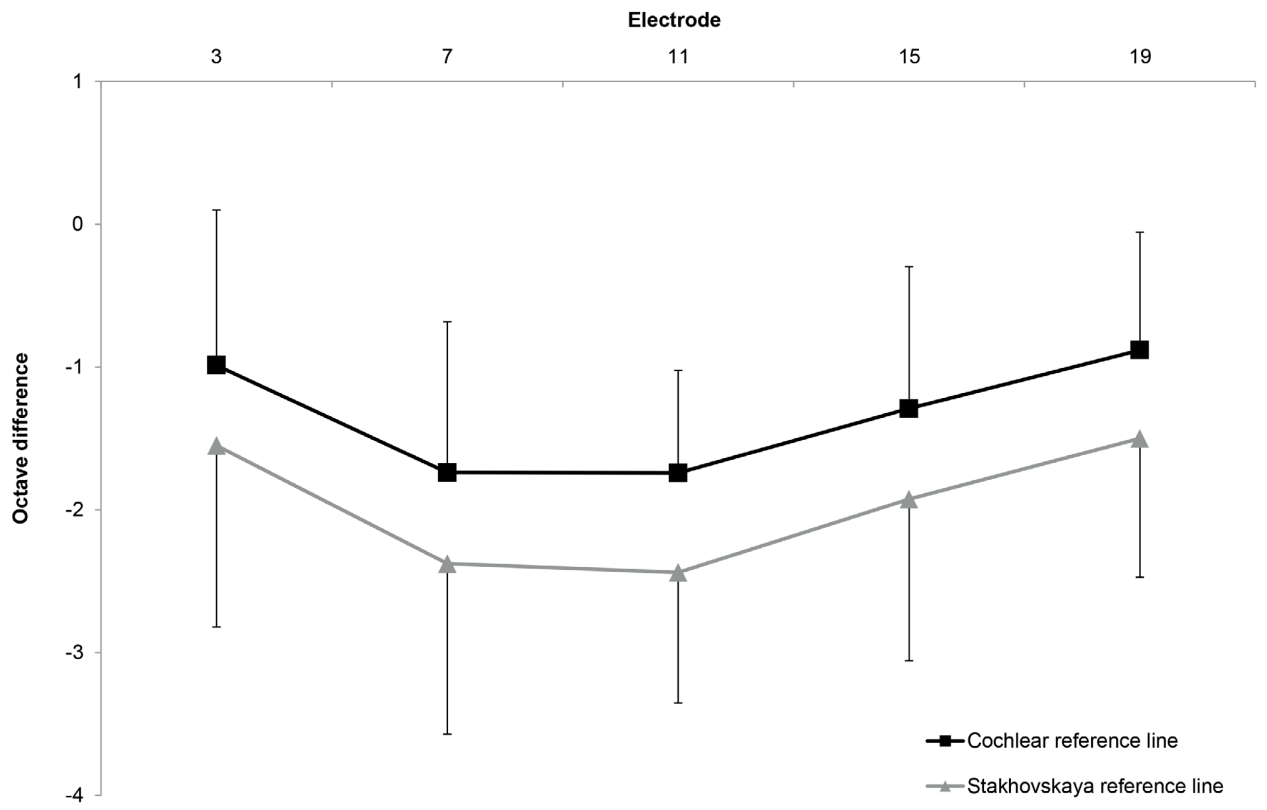

Legend: On the horizontal axis the electrode number is depicted, whereas the vertical axis represents the octave difference between the averaged line for patients and the Stakhovskaya reference line (grey triangles, grey line) and the Cochlear reference line (black squares, black line). Error bars represent standard deviations.

A weak correlation existed between the $\mathrm{PTA}_{\mathrm{be}}$ and the mismatch to the Stakhovskaya reference line $\left(R^{2}=0.41, P<0.05\right)$ and the Cochlear reference line $\left(R^{2}=0.52, P<0.05\right)$ (Figure 7). Strangely, this correlation shows that the higher the $\mathrm{PTA}_{\mathrm{be}}$ is, the smaller the mismatch; 
which is in contrast with what we would expect. The other patient characteristics (age, DoD and experience with CI) were not correlated to the mismatch (for all $P>0.1$; see Supplementary Material 4). Importantly, we observed that there was no significant correlation between performance (median CVC phoneme recognition scores 81\% (48-94\%), Table 1) and the mismatch to the Stakhovskaya reference line $\left(R^{2}=0.06, P>0.1\right)$ and the Cochlear reference line $\left(R^{2}=0.01, P>0.1\right)$.

Figure 7. Correlation between octave difference and Pure Tone Average of the better ear

Pure Tone Average of better ear (dB HL)

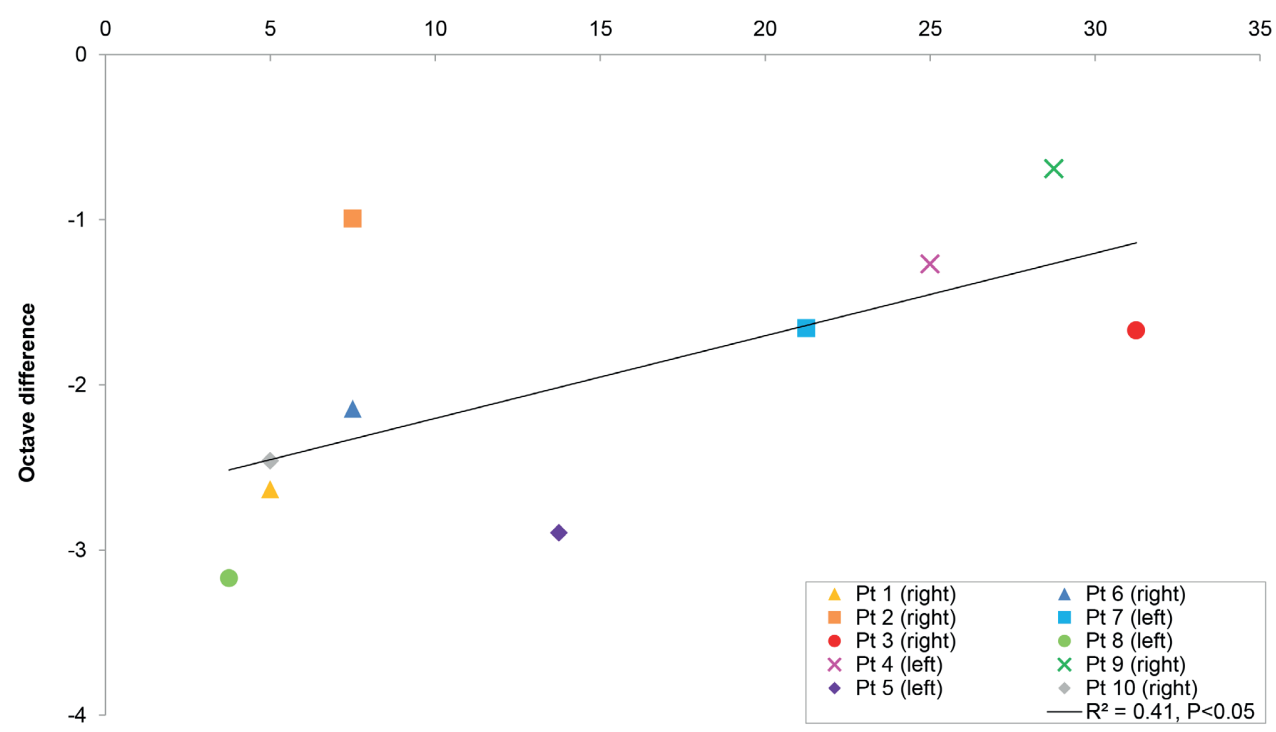

Legend: X-axis: Pure Tone Average of better ears (PTA ; $500,1000,2000,4000 \mathrm{~Hz}$ ), Y-axis: octave differences per patient relative to the Stakhovskaya reference line (data points relative to Cochlear reference line not depicted; see text for $R^{2}$ and $P$-values). The legend also denotes the side of the better ears. 


\section{DISCUSSION}

\section{Interpretation of results}

\section{Comparison with literature}

The mismatch of 2.0 octaves lower than the Stakhovskaya reference line and 1.3 octaves lower than the Cochlear reference line is similar to the mismatch observed by several other studies that investigated electro-acoustic pitch match in patients with residual hearing in the contralateral ear. ${ }^{2,5,6,11,12}$ There are several explanations for the observed mismatch to the Greenwood function ${ }^{4}$ (for organ of Corti) or Stakhovskaya's spiral ganglion cell map ${ }^{7}$, as outlined by Devocht et al. ${ }^{20}$ First, there could be methodological shortcomings in the pitch matching procedures: the results of pitch matching experiments may not have been reliable, for example when the "sanity checks" proposed by Carlyon et al. were not performed.22 These checks aim at discarding "unreliable pitch match data"; however, since it is unknown beforehand which data are reliable and which are not, we decided not to discard any data. Moreover, we think that a large intra- and intersubject variability is an important finding to report. Second, tonotopical reorganization may have occurred after CI activation, allowing the plastic neural system to get accustomed to the new frequency allocation. ${ }^{2,15}$ Consequently, pitch matching experiments do not reproduce the anatomic frequency allocation, but may result from a match at the level of the cortex in the reorganized CI hemisphere. Third, the degree of contralateral residual hearing may influence the pitch matches. ${ }^{16}$ In previous studies, patients often had moderate to severe hearing loss in the better ear., ${ }^{5,616}$ Our patient population had good hearing in the better ear ( $\mathrm{PTA}_{\mathrm{be}}$ maximum $30 \mathrm{~dB} \mathrm{HL}$ at time of inclusion). Still, we observed a weak correlation between the $\mathrm{PTA}_{\mathrm{be}}$ and the mismatch (Figure 7).

One additional explanation could be that the mismatch occurs because the electrical current between the electrode array and the extracochlear reference electrode finds its way via the trajectory of least resistance, which may differ from the histologically observed radial fiber trajectories as assumed by Stakhovskaya et al. ${ }^{7}$ Moreover, the extracochlear electrode may be positioned differently in every patient due to anatomical differences and surgical 
reasons, which also leads to different current trajectories.

In contrast, other studies found that the matched pitch was indeed similar to the Stakhovskaya reference line. ${ }^{16,22,23}$ Rader et al. (2016) observed so far unparalleled pitch matches for the lower frequency range in patients with SSD and a CI, by adapting the electric pulse rate to place-predicted frequency. ${ }^{23}$ Adjusting the pulse rate is however a completely different approach than our experimental setting.

Finally, we observed a large variance between maximum insertion angles of the most apical electrode (difference between the deepest and shallowest insertion of electrode 22 was $186^{\circ}$ ) in our patient group. This variance is in line with other reports on insertion angles ${ }^{8}$, even after correction for the added 34.6 degrees for the difference in definitions of the 0 -degree line. ${ }^{7,17}$ The insertion angle may be related to improved speech perception, since greatest benefits were observed for the deepest simulated CI insertion in some series, ${ }^{1,24}$ whereas others found insertion angle not to be a predicting factor for performance on word or phoneme recognition tasks. ${ }^{25}$

\section{$\underline{\text { Reliability of results }}$}

Pitch matching experiments are very ambiguous. ${ }^{26}$ It is a hard task for patients and requires a long attention span. A difficulty that all patients pointed out was that it was hard to compare the pitch of two unidentical stimuli: the sound of an activated electrode is not at all similar to a pure tone..$^{27}$ However, a $1 / 3$ noise band or warble tone did not make the comparison any easier for several of our patients. The difficulty of the task may have contributed to the observed large intra- and intersubject variability for the pitch matches.

We furthermore analyzed the data with the hypothesis of octave ambiguity. An octave ambiguity is a confusion between two tones with a difference of a plural of one octave. Octave ambiguities are also present in normal-hearing subjects. ${ }^{23,28}$ We calculated the octave differences between 1) the four runs per electrode per patient, and 2) the four runs per electrode and the Stakhovskaya and Cochlear reference lines per patient. We observed 
a uniform distribution of these octave differences for all electrodes, i.e. we did not observe a peak at exactly $-2.0,-1.0,1.0$ or 2.0 octaves. All individual data matches (runs $1-4$ ) per electrode per patients are presented in Supplementary Material 1 for more precise inspection of the octave differences. The absence of octave ambiguities is concordant with previous reports. ${ }^{11,23}$

Furthermore, we neither observed a bias established by the preceding signal or "sequential bias"22 (i.e. always a lower pitch match than the final geometric mean in the runs after a higher-numbered electrode had been tested just before).

\section{Methodological considerations}

There are several strengths that characterize our study. Our patients have good hearing in their better ear, resulting in a pitch perception that is identical to pitch perception of normalhearing cochlea. In contrast, most other published series considered patients with moderate to severe cochlear hearing loss in the "better" ear, resulting in a distorted pitch perception, ${ }^{13}$ which probably influenced their results. Furthermore, we determined the insertion angle based on HRCT scans by a semi-automatic in-house developed method, which yields better knowledge of the exact intracochlear electrode array position and insertion angles for specific electrode contacts.

We must also take some limitations of our approach into account. First, we did not match the loudness at all acoustic frequencies that we tested. We felt that this would have resulted in too much patient effort and thus compromise the willingness of patients to participate. Consequently, in our current results the pitch matches may have been influenced by (mainly high-frequency) hearing loss in the better ear. However, we know that the influence of loudness on pitch perception is negligible in the tested range (50-65 dB HL). ${ }^{19}$ Moreover, as noted previously, our patient sample had relatively good hearing in their better ear. Second, we did not present stimuli to the two ears alternatingly. Instead, we used an almost continuous electric stimulus. As a consequence, the loudness of the stimulus may have 
decreased in the CI ear hampering adequate matching. However, this is not very probable, because $200 \mathrm{~ms}$ silent gaps provided sufficient time for nerve condition restoration. Third, the duration of the acoustic stimulus was not fixed, but the researcher manually produced the tones via a computer and determined which tones were next to be presented. Finally, the order of electrode measurements (Supplementary Material 2) was identical for all patients and not randomized. Although we had good reasons not to randomize the order of electrode measurements, we cannot rule out the possible influence of a bias on the data.

\section{Clinical implications}

We observed a mismatch between the pitch match results from our patient group and the Stakhovskaya and Cochlear reference lines, as did previous articles. Interestingly, Landsberger et $a .^{8}$ adequately noted that there are three ways to deal with this mismatch: 1) by using electrode arrays that reach a deeper insertion angle, 2) by using different frequency-toelectrode translation tables, or 3) by letting patients adjust to the new frequency-place map hoping that neural plasticity will compensate for the frequency shift. However, it remains an

intriguing question whether the observed mismatch must lead to clinical consequences as listed above.

Some evidence points in the direction that it is indeed important to deliver auditory information at the correct cochlear place ${ }^{3,24}$, and changing the frequency allocation of the CI based on pitch match experiments or using different surgical insertion techniques may therefore be warranted. In contrast, others found that electrode position (i.e. correct tonotopic position) does not influence performance on word or phoneme recognition tasks. ${ }^{25}$ It might be the case that the neural system easily adapts to the new frequency-place situation, ${ }^{29}$ and consequently a mismatch hardly influences performance. In our sample, we observed no significant correlation between performance on a phoneme recognition test and the mismatch $\left(R^{2}=0.06, P>0.1\right)$. This contradicts the argumentative expectation that the phoneme scores might have been (even) higher, or asymptotic performance could have been reached earlier, 
if the observed mismatch between the pitch matches and the reference lines would have been smaller. ${ }^{24}$ However, for reliable pitch matches, patients they must first be accustomed to electric input through their CI. But as soon as there is electric input, reorganization of neural tissue occurs due to plasticity. ${ }^{8,30}$ Therefore, results of pitch matching experiments should be interpreted with caution.

\section{CONCLUSION}

Pitch matching experiments are very ambiguous. Methodologically, the pitch matching task is hard for patients. There is a large intra- and intersubject variability. Our results show that the pitch matches were 2.0 and 1.3 octaves lower than the Stakhovskaya and Cochlear reference lines, respectively. This mismatch is in line with most previous articles describing similar experiments. Given the methodological considerations, and the unclear correlation between the mismatch and performance on a phoneme recognition test, pitch matching results must not necessarily lead to altering the frequency allocation of the CI or to inserting the CI electrode array differently. 


\section{Author contributions}

JPMP: conception and design, acquisition of data, analysis and interpretation of data, drafting the article and revising it critically for important intellectual content, final approval of the version to be submitted.

EB: acquisition of data, analysis and interpretation of data, revising the article critically for important intellectual content, final approval of the version to be submitted.

WG: conception and design, revising the article critically for important intellectual content, final approval of the version to be submitted, principal investigator.

GAvZ: conception and design, analysis and interpretation of data, revising the article critically for important intellectual content, final approval of the version to be submitted.

\section{Conflict of interest}

Wilko Grolman received unrestricted research grants from Cochlear Ltd., Med-El GmbH and Advanced Bionics. The authors have no other funding, financial relationships or conflicts of interest to declare.

\section{Funding}

This study was partly funded by Cochlear Ltd. as an unrestricted research grant. By research contract, Cochlear Ltd. did not have influence on the study design, data collection, analysis, data interpretation, and publication. 


\section{SUPPLEMENTARY MATERIAL}

The supplementary material can be accessed online via http://dx.doi.org/10.1016/j. heares.2016.10.009.

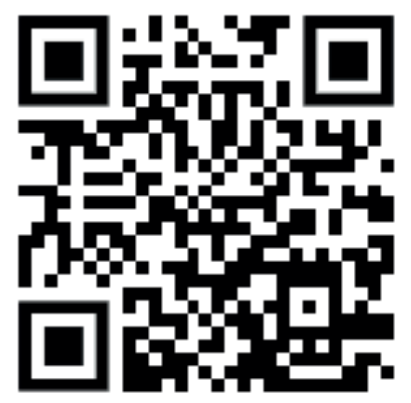

Supplementary Material 1: Supplementary data tables.

Supplementary Material 2: Order of electrode measurements.

Supplementary Material 3: Example of test procedure.

Supplementary Material 4: Results of statistical tests. 


\section{REFERENCES}

1. Buchman CA, Dillon MT, King ER, Adunka MC, Adunka OF, Pillsbury HC. Influence of cochlear implant insertion depth on performance: a prospective randomized trial. Otol Neurotol 2014;35(10):1773-1779.

2. Vermeire K, Landsberger DM, Van de Heyning PH, Voormolen M, Kleine Punte A, Schatzer R, Zierhofer C. Frequency-place map for electrical stimulation in cochlear implants: Change over time. Hear Res 2015;326:8-14.

3. Oxenham AJ, Bernstein JG, Penagos H. Correct tonotopic representation is necessary for complex pitch perception. Proc Natl Acad Sci USA 2004;101(5):1421-1425.

4. Greenwood DD. A cochlear frequency-position function for several species--29 years later. J Acoust Soc Am 1990;87(6):2592-2605.

5. Dorman MF, Spahr T, Gifford R, Loiselle L, McKarns S, Holden T, Skinner M, Finley C. An electric frequency-to-place map for a cochlear implant patient with hearing in the nonimplanted ear. J Assoc Res Otolaryngol 2007;8(2):234-240.

6. Boëx C, Baud L, Cosendai G, Sigrist A, Kós MI, Pelizzone M. Acoustic to electric pitch comparisons in cochlear implant subjects with residual hearing. J Assoc Res Otolaryngol 2006;7(2):110-124.

7. Stakhovskaya O, Sridhar D, Bonham BH, Leake PA. Frequency map for the human cochlear spiral ganglion: implications for cochlear implants. J Assoc Res Otolaryngol 2007;8(2):220-233.

8. Landsberger DM, Svrakic M, Roland JT Jr, Svirsky M. The Relationship Between Insertion Angles, Default Frequency Allocations, and Spiral Ganglion Place Pitch in Cochlear Implants. Ear Hear 2015;36(5):e207-213.

9. Erixon E, Rask-Andersen H. How to predict cochlear length before cochlear implantation surgery. Acta Otolaryngol 2013;133(12):1258-1265.

10. Van der Marel KS, Briaire JJ, Wolterbeek R, Snel-Bongers J, Verbist BM, Frijns JHM. Diversity in cochlear morphology and its influence on cochlear implant electrode 
position. Ear Hear 2014;35(1):e9-20.

11. Baumann U, Nobbe A. The cochlear implant electrode-pitch function. Hear Res 2006;213(1-2):34-42.

12. Schatzer R, Vermeire K, Visser D, Krenmayr A, Kals M, Voormolen M, Van de Heyning P, Zierhofer C. Electric-acoustic pitch comparisons in single-sided-deaf cochlear implant users: frequency-place functions and rate pitch. Hear Res 2014;309:26-35.

13. Gaeth JH, Norris TW. Diplacusis in unilateral high-frequency hearing losses. J Speech Hear Res 1965;8:63-75.

14. Xu J, Xu SA, Cohen LT, Clark GM. Cochlear view: postoperative radiography for cochlear implantation. Am J Otol 2000;21:49-56.

15. McDermott H, Sucher C, Simpson A. Electro-acoustic stimulation. Acoustic and electric pitch comparisons. Audiol Neurotol 2009;14(Suppl 1):2-7.

16. Vermeire K, Nobbe A, Schleich P, Nopp P, Voormolen MH, Van de Heyning PH. Neural tonotopy in cochlear implants: an evaluation in unilateral cochlear implant patients with unilateral deafness and tinnitus. Hear Res 2008;245(1-2):98-106.

17. Strange P. The sense of pitch and local increase in threshold. J Physiol 1955;129(2):225236.

18. Verbist BM, Joemai RMS, Briaire JJ, Teeuwisse WM, Veldkamp WJH, Frijns JHM. Cochlear coordinates in regard to cochlear implantation: a clinically individually applicable 3 dimensional CT-based method. Otol Neurotol 2010;31(5):738-744.

19. Peters JPM, Van Zon A, Smit AL, Van Zanten GA, De Wit GA, Stegeman I, Grolman W. CINGLE-trial: Cochlear Implantation for siNGLE-sided deafness, a Randomized controlled trial and economic evaluation. BMC Ear, Nose and Throat Disorders 2015;15:3.

20. Verbist BM, Skinner MW, Cohen LT, Leake PA, James C, Boëx C, Holden TA, Finley CC, Roland PS, Roland JT Jr, Haller M, Patrick JF, Jolly CN, Faltys MA, Briaire JJ, Frijns JHM. Consensus panel on a cochlear coordinate system applicable in histologic, physiologic, and radiologic studies of the human cochlea. Otol Neurotol 2010;31(5):722-730. 
21. Devocht EM, Dees G, Arts RA, Smits JJ, George EL, van Hoof M, Stokroos RJ. Revisiting Place-Pitch Match in CI Recipients Using 3D Imaging Analysis. Ann Otol Rhinol Laryngol 2016;125(5):378-384.

22. Carlyon RP, Macherey O, Frijns JH, Axon PR, Kalkman RK, Boyle P, Baguley DM, Briggs J, Deeks JM, Briaire JJ, Barreau X, Dauman R. Pitch comparisons between electrical stimulation of a cochlear implant and acoustic stimuli presented to a normal-hearing contralateral ear. J Assoc Res Otolaryngol 2010;11(4):625-640.

23. Rader T, Döge J, Adel Y, Weissgerber T, Baumann U. Place dependent stimulation rates improve pitch perception in cochlear implantees with single-sided deafness. Hear Res 2016;339:94-103.

24. Zhou X, Li H, Galvin JJ 3rd, Fu QJ, Yuan W. Effects of insertion depth on spatial speech perception in noise for simulations of cochlear implants and single-sided deafness. Int J Audiol 2016;1:1-8.

25. Van der Marel KS, Briaire JJ, Verbist BM, Muurling TJ, Frijns JHM. The influence of cochlear implant electrode position on performance. Audiol Neurotol 2015;20(3):202211.

26. Van den Brink G. On the relativity of pitch. Perception 1982;11(6):721-731.

27. Lazard DS, Marozeau J, McDermott HJ. The sound sensation of apical electric stimulation in cochlear implant recipients with contralateral residual hearing. PLoS One 2012;7(6):e38687.

28. Borra T, Versnel H, Kemner C, van Opstal AJ, van Ee R. Octave effect in auditory attention. Proc Natl Acad Sci U S A 2013;17;110(38):15225-15230.

29. Eggermont JJ. Acquired hearing loss and brain plasticity. Hear Res 2017;343:176-190.

30. Zeng FG, Tang Q, Lu T. Abnormal pitch perception produced by cochlear implant stimulation. PLoS One 2014;9(2):e88662. 


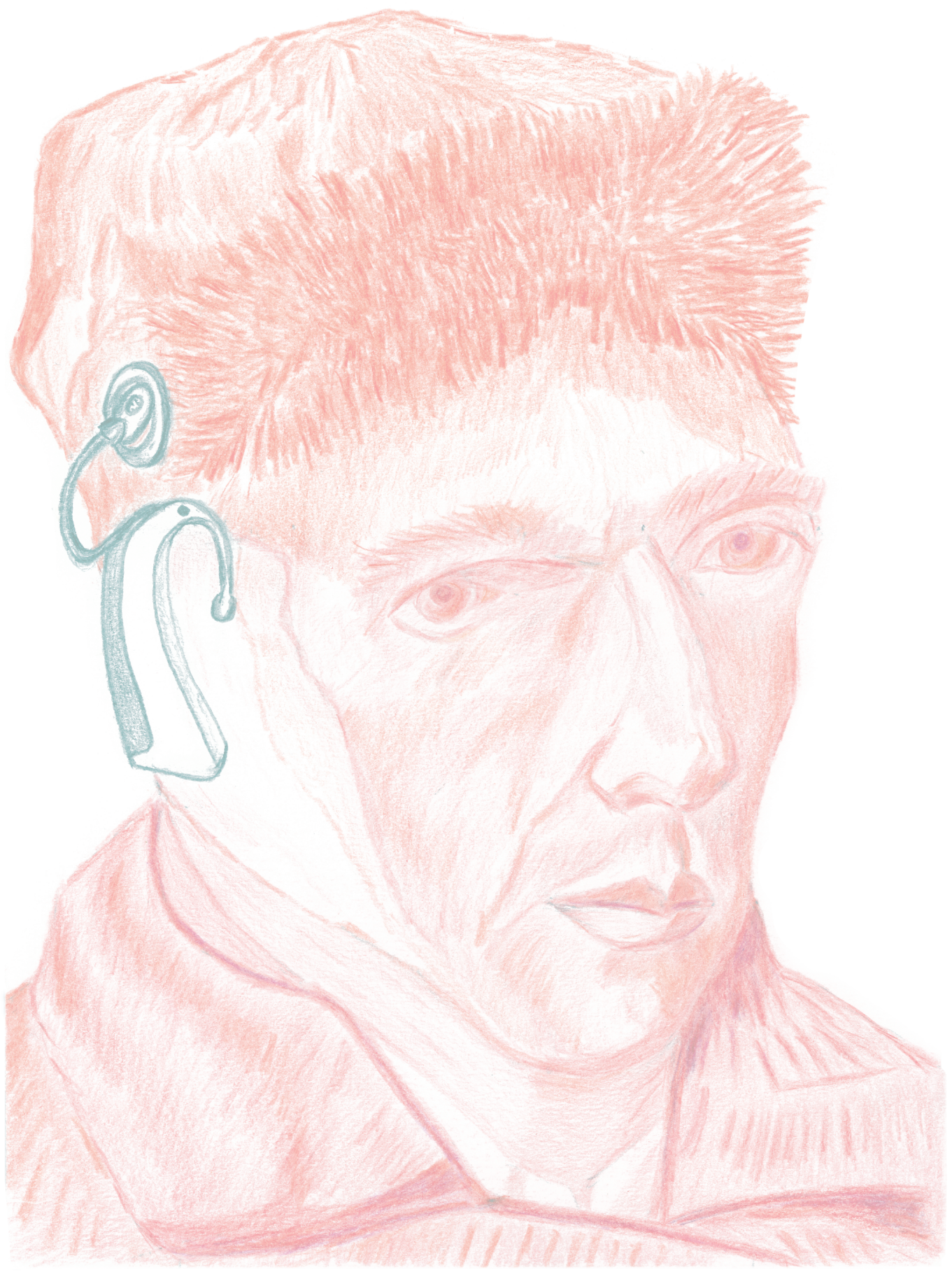




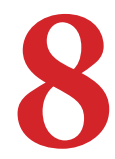

\section{Comparison of Place-versus-Pitch Mismatch between a Perimodiolar and Lateral Wall Cochlear Implant Electrode Array in Patients with Single-Sided Deafness and a Cochlear Implant}




\section{ABSTRACT}

\section{Background}

In electric-acoustic pitch matching experiments in patients with single-sided deafness (SSD) and a cochlear implant (CI), the observed "mismatch" between perceived pitch and predicted pitch based on the amended Greenwood frequency map, ranges from -1 to -2 octaves. It is unknown if and how this mismatch differs for perimodiolar versus lateral wall electrode arrays.

\section{Objectives}

We aimed to investigate if the type of electrode array design is of influence on the electricacoustic pitch match.

\section{Method}

Fourteen patients $(n=8$ with CI422 + lateral wall electrode array, $n=6$ with CI512 + perimodiolar electrode array; Cochlear Ltd.) compared the pitch of acoustic stimuli to the pitch of electric stimuli at two test sessions (average interval 4.3 months). We plotted these "pitch matches" per electrode contact against insertion angle, calculated from high-resolution computed tomography scans. The difference between these pitch matches and two references (the spiral ganglion map and the default frequency allocation by Cochlear Ltd.) was defined as "mismatch".

\section{Results}

We found average mismatches of -2.2 octave for the CI422 group and - 1.3 octave for the CI512 group. For any given electrode contact, the mismatch was smaller for the CI512 electrode array than for the CI422 electrode array. For all electrode contacts together, there was a significant difference between the mismatches of the two groups $(p<0.05)$. Results remained stable over time, with no significant difference between the two test sessions considering all 
electrode contacts. Neither group showed a significant correlation between the mismatch and phoneme recognition scores.

\section{Conclusion}

The pitch mismatch was smaller for the perimodiolar electrode array than for the lateral wall electrode array. 


\section{INTRODUCTION}

A cochlear implant (CI) is an auditory prosthesis that partially restores auditory input in case of a deafened cochlea. A CI has an electrode array, which is surgically placed in the cochlea and distributes auditory information tonotopically: the lowest frequencies to the deepest inserted electrode contact (for all CI brands a center frequency of about $200 \mathrm{~Hz}$ ), the highest frequencies to the shallowest inserted electrode contact $(8,000 \mathrm{~Hz})$. The CI thereby mimics the tonotopy of auditory information of a normal ear. Patients with single-sided deafness (SSD) and a CI must fuse the electrical input from their CI with the acoustic input from their better ear. A possible mismatch in pitch between ears may have detrimental effects on the fusion of the signals from the two ears, which may limit performance. ${ }^{1,2}$

As the electrical stimulus activates spiral ganglion cells and not the organ of Corti, the classical place-pitch relation ${ }^{3}$ has been amended for CI stimulation..$^{4-6}$ In pitch matching experiments in SSD patients implanted with a CI, the perceived pitch is 1 to 2 octaves lower than the pitch predicted by the amended place-pitch relation. ${ }^{4,7-9}$ Some of this mismatch may be due to the fact that in several studies ${ }^{2,4,7-11}$ the determination of the electrode array's position was inaccurate, due to the use of planar X-ray imaging. ${ }^{12}$ Furthermore, the better ear in these patients was moderately or severely impaired, possibly resulting in distorted pitch perception. $^{13}$

In a previous paper, we described electric-acoustic pitch match experiments in ten patients with SSD and a CI. ${ }^{14}$ In contrast to previous studies ${ }^{4,7-9}$, we determined the insertion angle of the electrode array using high resolution computed tomography (HRCT) scans by a semiautomatic in-house developed method. ${ }^{15}$ This method yields better knowledge of the exact intracochlear electrode array position and insertion angles for specific electrode contacts compared to planar X-ray imaging. Additionally, we performed our experiments in a group of patients with near-normal hearing in their better ear, decreasing the risk of distorted pitch perception. ${ }^{13}$ Even with these optimized methods, we also found a mismatch of about 2 octaves compared to the histological findings in human temporal bones of Stakhovskaya 
et $a .^{5}$ We also explored if the mismatch was of influence on performance on a phoneme recognition test and found that they were not correlated. ${ }^{14}$

In the current paper, we aimed to investigate if the type of electrode array design is of influence on the electric-acoustic pitch match. We compared lateral wall electrode arrays and perimodiolar electrode arrays, with greater or smaller distance between the electrode array and spiral ganglion cells (located in the modiolus), respectively. The influence of the electrode array design in relation to performance has been investigated ${ }^{16,17}$ : some authors concluded that a lateral wall electrode array led to better performance ${ }^{18}$, whereas others found that proximity to the modiolus (i.e. perimodiolar) is correlated with better performance. ${ }^{19-21}$ Van der Beek et al. hypothesized that with the electrode array closer to the modiolus, "spatial selectivity" is enhanced: electrode contacts in close proximity to the spiral ganglion cells have a better chance of stimulating a more narrow tonotopic region of the cochlea than electrode contacts that are located farther away. ${ }^{21}$ Consequently, there is a better discrimination between electrode contacts that leads to improved speech perception. ${ }^{19}$ Additionally, we performed a re-test after the initial experiment, to investigate if the results of the electric-acoustic pitch match experiments were stable over time, like they were in the experiments conducted by Vermeire et al. ${ }^{2}$

\section{METHODS}

A large part of the methods we used was identical to the methods that we detailed in Peters et al. ${ }^{14}$ We will briefly repeat these methods, and elaborate where new methods were used.

\section{Patients}

Fourteen patients with SSD and a CI participated in the current experiment, divided into two groups. The "CI422" group consisted of eight patients implanted with a CI with a lateral wall electrode array (Cochlear ${ }^{\circledR}$ CI422, Slim Straight electrode array). The "CI512" group consisted of six patients implanted with a CI with a perimodiolar electrode array (Cochlear ${ }^{\circledast}$ 
CI512, Contour Advance electrode array). All patients provided Informed Consent for participation in this study, which was ethically approved by the Institutional Review Board of the University Medical Center Utrecht (NL45288.041.13; and its later amendments). One of the inclusion criteria of the study was a Pure Tone Average threshold in the better ear $\left(\mathrm{PTA}_{\mathrm{be}}\right.$; $500,1000,2000$ and $4000 \mathrm{~Hz}$ ) of maximum $30 \mathrm{~dB}$ HL in the better ear, and of at least $70 \mathrm{~dB}$ HL in the poor ear. For details about other inclusion and exclusion criteria, see the study protocol. ${ }^{22}$ All patient characteristics are summarized in Table $\mathbf{1 .}$

Table 1. Patient characteristics of the included patients

\begin{tabular}{|c|c|c|c|c|c|c|c|c|c|c|}
\hline $\begin{array}{l}\text { CI } \\
\text { type }\end{array}$ & Patient & Gender & $\begin{array}{l}\text { Age at } \\
\text { CI acti- } \\
\text { vation } \\
\text { (years) }\end{array}$ & $\begin{array}{l}\text { Deaf } \\
\text { Ear }\end{array}$ & Etiology & $\begin{array}{l}\text { Dura- } \\
\text { tion of } \\
\text { deafness } \\
\text { (years) }\end{array}$ & $\begin{array}{l}\text { PTA bet- } \\
\text { ter ear } \\
(0.5-4 \\
\text { kHz) (dB } \\
\text { HL) }\end{array}$ & $\begin{array}{l}\text { Expe- } \\
\text { rience } \\
\text { at Test } \\
\text { (months) }\end{array}$ & $\begin{array}{l}\text { Experi- } \\
\text { ence at } \\
\text { Re-test } \\
\text { (months) }\end{array}$ & $\begin{array}{l}\text { CVC } \\
\text { pho- } \\
\text { neme } \\
\text { score }^{\star}\end{array}$ \\
\hline \multirow{8}{*}{ CI422 } & Pt 1 & M & 67.4 & Left & Labyrinthitis & 3.3 & 31.3 & 6.3 & 16.6 & $48 \%$ \\
\hline & Pt 2 & M & 58.5 & Right & Unknown & 9.9 & 13.8 & 3.4 & 9.9 & $59 \%$ \\
\hline & Pt 3 & M & 47.0 & Left & Ménière’s disease & 4.7 & 7.5 & 3.1 & 5.9 & $83 \%$ \\
\hline & Pt 4 & M & 37.5 & Right & Unknown & 3.2 & 21.3 & 6.5 & 12.0 & $93 \%$ \\
\hline & Pt 5 & M & 64.1 & Left & Unknown & 4.2 & 28.8 & 3.2 & 7.7 & $94 \%$ \\
\hline & Pt 6 & $\mathrm{~F}$ & 23.9 & Left & Sudden deafness & 1.7 & 5.0 & 3.4 & 6.7 & $78 \%$ \\
\hline & Pt 7 & $\mathrm{~F}$ & 73.1 & Left & Labyrinthitis & 1.1 & 13.8 & 3.0 & 6.6 & $65 \%$ \\
\hline & Pt 8 & $\mathrm{~F}$ & 43.5 & Left & Sudden deafness & 2.0 & 10.0 & 3.4 & 6.9 & $26 \%$ \\
\hline \multirow{6}{*}{ CI512 } & Pt 9 & $\mathrm{~F}$ & 46.5 & Left & Sudden deafness & 8.2 & 15.0 & 2.9 & 6.5 & $64 \%$ \\
\hline & Pt 10 & $\mathrm{~F}$ & 38.0 & Right & Sudden deafness & 0.9 & 8.8 & 3.2 & 6.9 & $86 \%$ \\
\hline & Pt 11 & M & 65.1 & Right & Sudden deafness & 1.0 & 18.8 & 3.2 & 5.9 & $65 \%$ \\
\hline & Pt 12 & $\mathrm{~F}$ & 56.4 & Left & Sudden deafness & 0.9 & 5.0 & 2.5 & 6.4 & $89 \%$ \\
\hline & Pt 13 & M & 58.7 & Right & Unknown & 5.9 & 18.8 & 3.4 & 5.9 & $61 \%$ \\
\hline & Pt 14 & $\mathrm{~F}$ & 61.3 & Left & Sudden deafness & 1.0 & 21.3 & 2.7 & 6.7 & $89 \%$ \\
\hline
\end{tabular}

\section{Legend:}

PTA $=$ Pure Tone Average $(500,1000,2000$ and $4000 \mathrm{~Hz})$.

${ }^{\star} \mathrm{CVC}=$ Consonant-Vowel-Consonant phoneme score at 3 months after CI activation for the CI-ear only (better ear masked with masking noise).

Patient 1 (Pt 1) had a PTA of the better ear of $30.0 \mathrm{~dB}$ HL at time of inclusion, but his hearing slightly deteriorated.

All patients were tested twice, first after a median duration of 3.2 months after CI activation (“Test”), and a second time after a median duration of 6.7 months after CI activation ("Re- 
test"). The average time between the Test and Re-test session was 4.3 months. Of the eight patients in the CI422 group, the data of the Test session of the first six patients were also presented in our previous paper. ${ }^{14}$ In Figure 1, the average hearing thresholds for the better ear are displayed per group for all frequencies 125-8000 Hz. There was no significant difference between the $\mathrm{PTA}_{\mathrm{be}}$ of the CI422 group compared to the CI512 group (Mann-Whitney U test, $\mathrm{p}=0.897)$.

Figure 1. Average pure tone hearing thresholds (dB HL) per group. Error bars represent standard deviations.

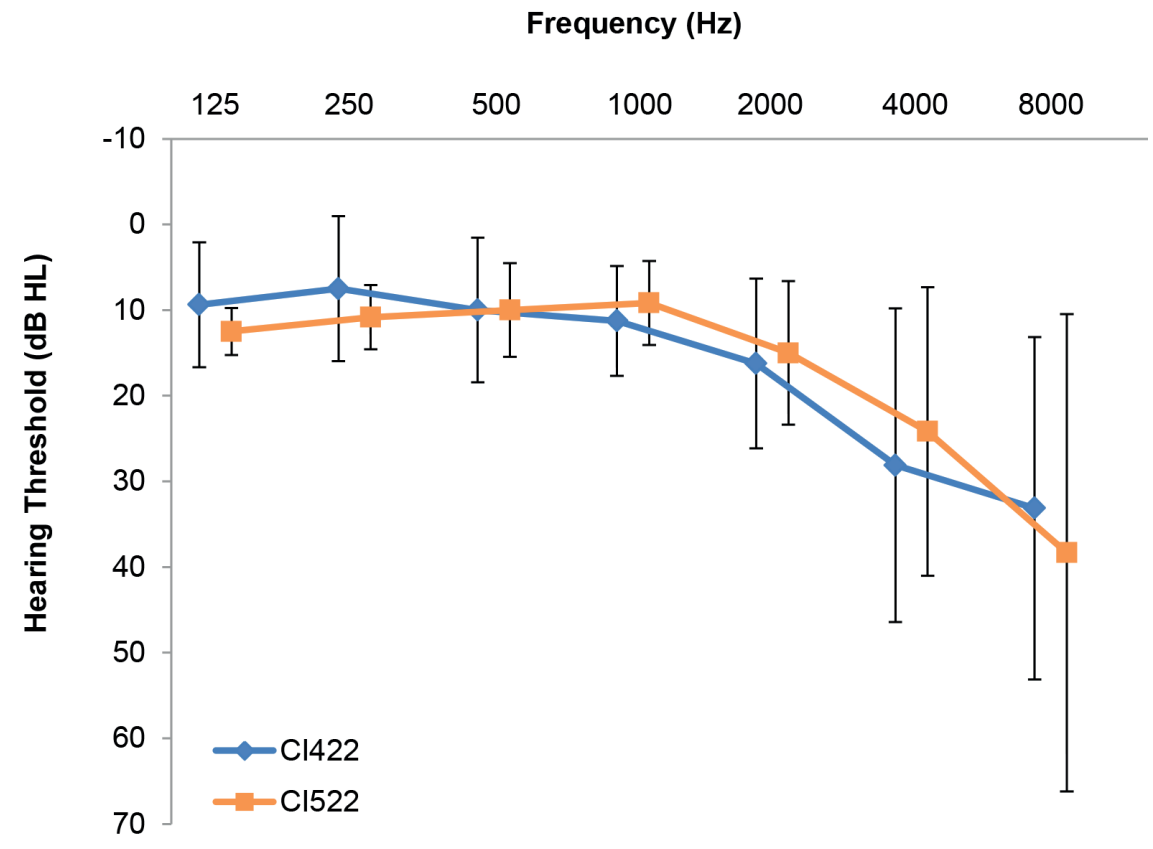

All patients were satisfied with their CI and wore the device often, except Pt 8 (CI422 group with Pt 8: median use 8.8 h/day, without Pt 8: 12.1 h/day; CI512 group: 12.1 h/day).There was no significant difference between groups with or without Pt 8 (Mann-Whitney U test, $p>.05)$. Pt 8 reported that she could not get used to the 'shrill' and 'electric' sound of the $\mathrm{CI}$, even after many attempts to personalize the frequency mapping, including switching off 
several electrode contacts. She performed poorer than expected (Table 1). Nevertheless, she did participate in Test and Re-test experiments. One year after implantation, she became a non-user.

\section{Cochlear Implants}

The Cochlear ${ }^{\oplus}$ CI422 has a Slim Straight electrode array, which is a lateral wall electrode array. The Cochlear ${ }^{\circledast}$ CI512 has a Contour Advance electrode array, which is a perimodiolar electrode array. Both electrode array types consist of 22 electrode contacts: electrode contact number 1 (E1) is located most basally (high frequency tones) in the cochlea, and E22 is located most apically (low frequency tones). The allocated center frequencies per electrode contact are identical for the CI422 and the CI512. The dimensions of both electrode array types and the frequency allocation per electrode contact are depicted in Table 2 . Note that the CI512 Contour Advance electrode array $(11.70 \mathrm{~mm})$ is shorter than the CI422 Slim Straight electrode array $(18.75 \mathrm{~mm})$. 


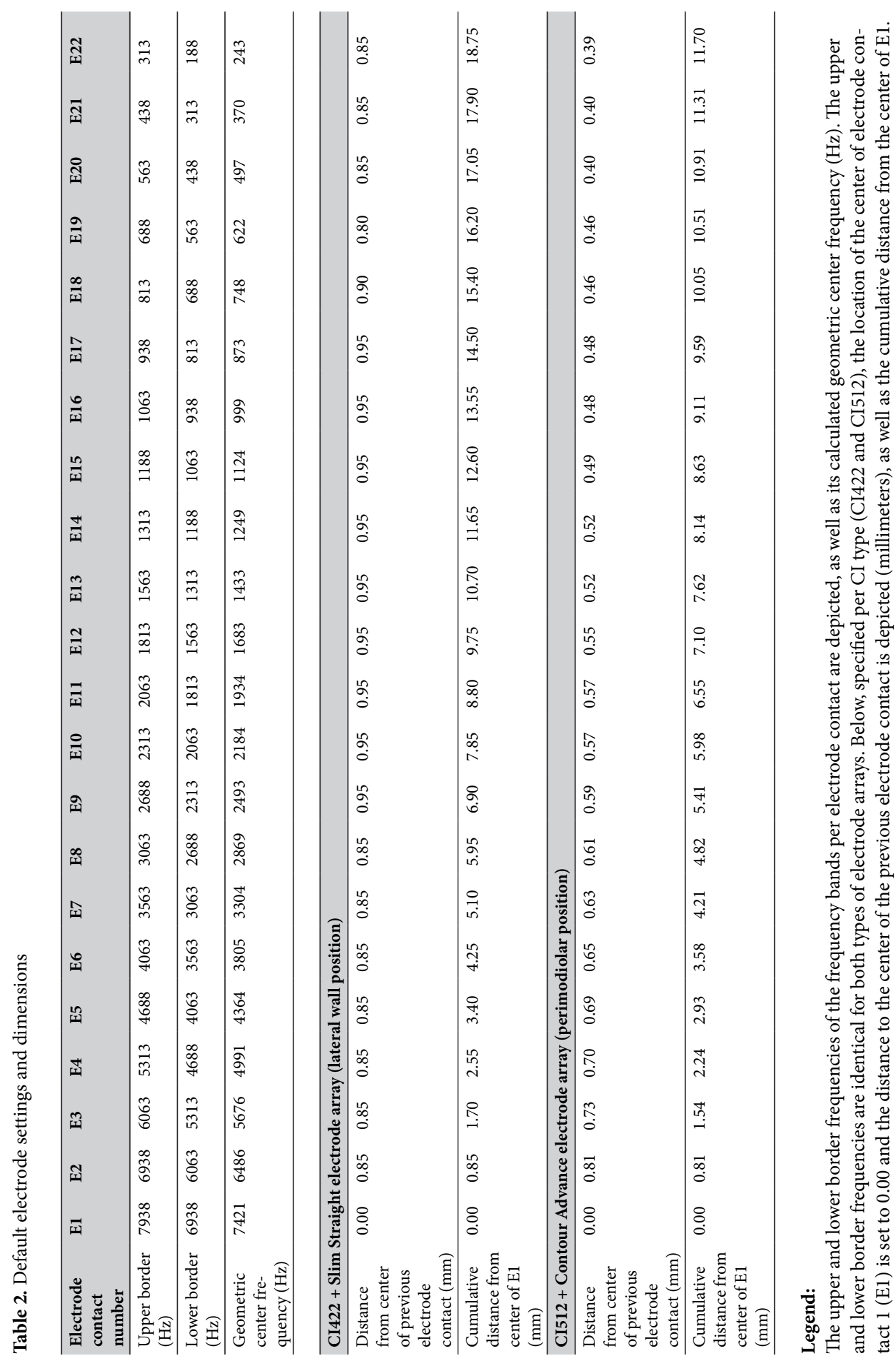


For some patients (two patients in CI422 group, two patients in CI512 group), specific electrode contacts were switched off during CI rehabilitation because of high impedances. Consequently, the center frequencies of the remaining active electrode contacts differed from default because of the automatic reallocation (see Supplementary Material 1 for frequency allocations per patient). The geometric mean of the center frequencies per electrode contact of all patients is referred to as the "Cochlear reference line" in this paper.

\section{Stimuli and experimental design}

The experiments took place in an acoustically insulated, single-walled and internally heavily damped room (IAC Acoustics $\mathrm{GmbH}$, Niederkrüchten, Germany), with noise reduction coefficient $>0.8$, inside a custom built already quiet room. To be able to use the data from our previous experiment, we used the same electric and acoustic stimuli in this experiment. ${ }^{14}$ In short, we electrically stimulated five electrode contacts separately (E3, E7, E11, E15, E19) four times in all patients at a comfortable level (C-level of the most frequently used program). The electric stimulus consisted of a continuously repeated biphasic pulse train (pulse duration $25 \mu$ s, pulse repetition rate $900 \mathrm{~Hz}$, train duration $5000 \mathrm{~ms}$ ) followed by a silent interval of $200 \mathrm{~ms}$. The acoustic stimuli consisted of pure tones, generated by a clinical audiometer and presented from a loudspeaker at $110 \mathrm{~cm}$ height (head level in seated position) and $130 \mathrm{~cm}$ distance in front of the patient. The manually-presented acoustic stimuli had a minimum duration of $500 \mathrm{~ms}$. For the first run of measurements per electrode, we adjusted the level of a $1 \mathrm{kHz}$ acoustic tone (median $60 \mathrm{~dB} \mathrm{HL}$, range $50-65 \mathrm{~dB} \mathrm{HL}$ ) to match it to the loudness of the electric stimulus in the CI ear (C-level of the most frequently used program). In this range $(50-65 \mathrm{~dB} \mathrm{HL})$, the influence of loudness on pitch perception is negligible. ${ }^{23}$ The task was to compare the pitch of two acoustic stimuli to the pitch of the electric stimulus, and select the acoustic stimulus with greatest similarity in pitch. The difference between the two acoustic tones gradually decreased ( 2 octaves to $1 / 8$ octave), to end at the acoustic stimulus with a pitch "the most similar" to the pitch of the electric stimulus. We then calculated the 
geometric mean of the matched frequencies per electrode contact per patient ("pitch match").

\section{Insertion angle of CI electrode array}

The intracochlear electrode array position was imaged using postoperative HRCT scans. Image acquisition and analysis are detailed in Bennink et al. ${ }^{15}$ In short, each HRCT scan was filtered, and a 3D bounding box was manually positioned on the cochlea, in which the centerline of the electrode array was automatically tracked. The variation in CT values along this centerline was used to determine the electrode contact positions. The correlation of the CI geometry, as described in Table 2, with the variation in CT values was calculated at varying positions along the centerline. Since the CI512 is a preshaped perimodiolar implant, the correlation was also calculated for a varying stretch (0 to $15 \%)$ with respect to its specified dimensions, whereas for the CI422 a compression (0 to 10\%) was allowed. The results were visually verified on a $4 \mathrm{~mm}$ thick reformatted projection of the scan, and another local optimum was chosen if the global optimum was found to be incorrect.

The transformation of the resulting CT coordinates to a cylindrical coordinate system (insertion angle, radius, elevation) requires fitting a plane through the basal turn of the cochlea, and the manual selection of two reference points. ${ }^{24}$ The selection of these two reference points (the top of the modiolus and the most lateral point of the horizontal semicircular canal) was carried out by an experienced radiologist. The top of the modiolus defines the center of the cylindrical coordinate system, whereas the line between the two reference points defines the $-34.6^{\circ}$ angle with respect to the position of the round window. ${ }^{25}$

With knowledge of the insertion angle per electrode contact for all patients (see Supplemental Material 1), we could calculate the predicted frequency using the Stakhovskaya histology results. ${ }^{5}$ This predicted frequency is referred to as the "Stakhovskaya reference line" in this paper.

We expressed the intracochlear electrode array insertion as insertion angle instead of insertion depth, because the size of the cochlea may differ amongst patients ${ }^{5,26,27}$ and 
the intracochlear position of the electrode array may lead to different insertion angles for identical insertion depths.

\section{Data analysis}

We compared the mismatch between the pitch matches of our patients to the Stakhovskaya reference line and the Cochlear reference line. We analyzed this mismatch per group (CI422 and CI512) and per experiment (Test and Re-test). A two-sided one sample t-test was used to test if the pitch matches per electrode contact differed significantly from the reference lines. For between-group comparisons, the Mann-Whitney U test was used; for within-group comparisons, the Wilcoxon signed-rank test was used.

Additionally, we checked if a correlation existed between the mismatch and the patient characteristics listed in Table 1 (age, duration of deafness, experience with CI, $\left.\mathrm{PTA}_{\mathrm{be}}\right)$. Finally, we also checked if there was a correlation between the mismatch and the performance on a speech recognition task (Consonant-Vowel-Consonant (CVC) phoneme recognition scores 3 months after CI activation for the CI-ear only (better ear masked with speech-shaped noise, presented via an insert earphone, at a speech-to-masking level ratio of $-10 \mathrm{~dB}$ or worse)); see Table 1 . The standard Dutch CVC phoneme recognition task was used, in which eleven CVC words with 33 phonemes per list are presented. ${ }^{28}$ The lists were presented in a free field condition at $65 \mathrm{~dB}$ SPL. Two lists were presented in order to improve measurement accuracy of the CVC-score.

Data analysis was performed using SPSS Statistics software version 22. A p-value of $<0.05$ was considered statistically significant.

\section{RESULTS}

\section{Intracochlear electrode array position}

Figure 2a shows the radius (distance from electrode contact to the modiolus axis) relative to the insertion angle per group. The CI512 electrode array is shorter than the CI422 electrode 
array (dimensions presented in Table 2), and thus shallower inserted in the cochlea. The median insertion angle of E22 for the CI512 group was $307.3^{\circ}$, whereas the median insertion angle of E22 for the CI422 group was $399.7^{\circ}$. The radius of the CI512 electrode array was smaller than the radius of the CI422 electrode array for corresponding electrode contacts, which can be attributed to the preformed curvature of the CI512 electrode array.

Figure 2a. Average radius versus insertion angle per group.

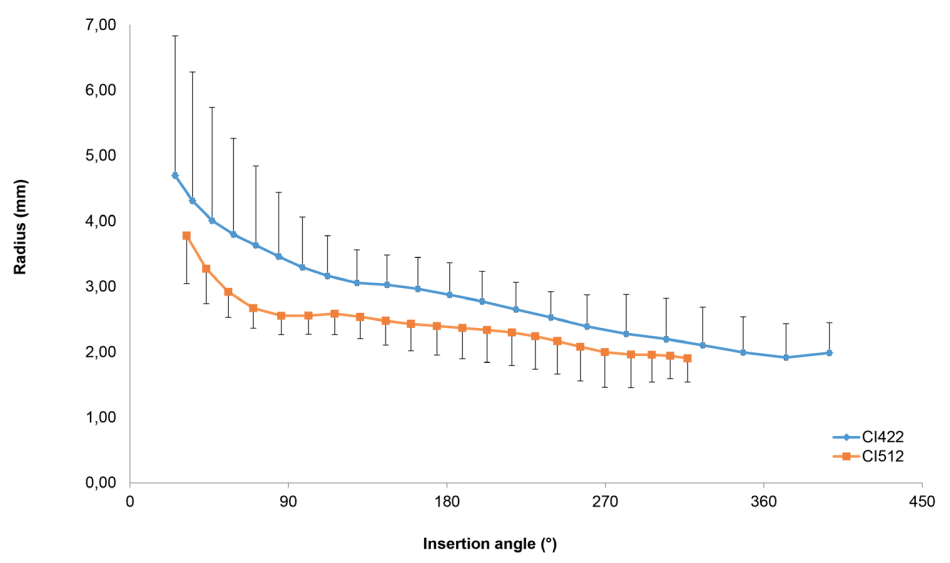

Legend: When inserted, the radius (distance from electrode contact to modiolus axis) of the CI512 electrode array was smaller than the radius of the CI422 electrode array at the same insertion angle. The electrode array of the CI512 is shorter than the CI422 electrode array (see also Table 2). Error bars represent standard deviations.

Figure 2b. Average elevation versus insertion angle per group.

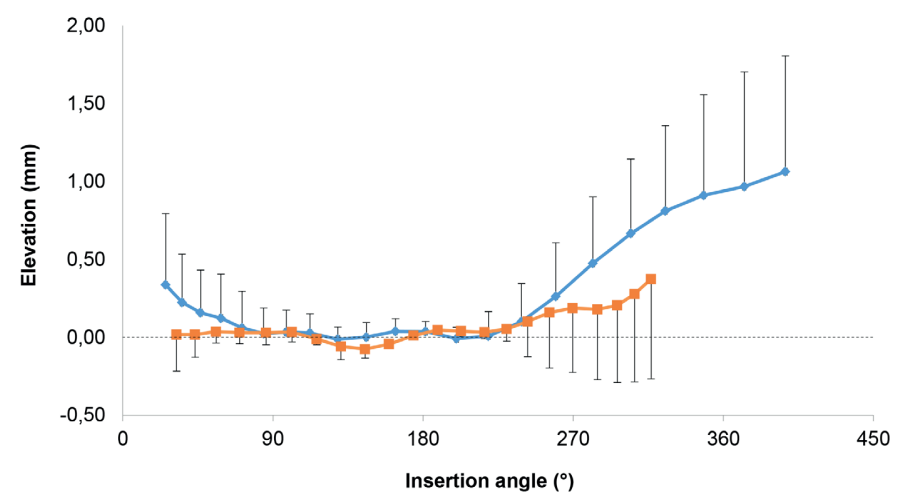

Legend: The maximum elevation (from the basal turn of the cochlea) of the deepest inserted electrode contact (E22) was smaller for the CI512 electrode array than for the CI422 electrode array. The electrode array of the CI512 is shorter than the CI422 electrode array (see also Table 2). Error bars represent standard deviations. 
In Figure $\mathbf{2} \mathbf{b}$, the elevation of the electrode contacts from the basal turn of the cochlea was plotted versus insertion angle per group. Again, we can observe that the CI512 electrode array is shorter, and shallower inserted. Because of its shorter length, it did not reach as high into the second cochlear turn as the CI422 electrode array (averaged maximum elevation of E22 for CI512 group: $0.37 \mathrm{~mm}$, for CI422 group: $1.06 \mathrm{~mm}$ ).

Insertion angles showed great variability in our population, not only between groups, but also between individual patients. The electrode array of Pt 8 (CI422 group) was inserted very shallow (insertion angle E22: $235.0^{\circ}$ ), resulting in 4-5 electrode contacts positioned outside the cochlea. This may have contributed to her poor performance, dissatisfaction and finally becoming a non-user. Because E3 was positioned outside the cochlea, we did not use the insertion angle of this electrode contact for this patient in our calculations. In contrast, the electrode array of Pt 14 (CI512 group) was positioned deep in the cochlea, with E22 positioned at $421.4^{\circ}$ (relatively deep for CI512 group). For all individual data on insertion angles per electrode contact, see Supplemental Material 1.

\section{Pitch match}

In total, we presented 8,169 comparisons to fourteen patients in two experiments consisting of four runs on five electrode contacts, resulting in a mean ( \pm standard deviation, SD) of 14.8 \pm 3.6 comparisons per patient per run per electrode contact per experiment. For a closer inspection of all individual measurements, please see Supplemental Material 1. Like in our previous experiment, we noticed that the task was often difficult for patients. ${ }^{14}$ The difficulty of the task may have contributed to the observed large intra- and intersubject variability, which was comparable to our previous observations. As discussed in our previous report, we again observed no octave ambiguities (in a two-tone comparison, octave ambiguity is the identification by a listener of a higher harmonic as the fundamental tone, which means that a frequency at integer multiple value of fundamental frequency is confounded with the fundamental frequency itself), which was also concordant with other series. ${ }^{8,29}$ 
In Figure 3, the averaged pitch matches per group and per experiment are depicted for electrode contacts E3, E7, E11, E15, and E19. The logarithmic vertical axis shows frequency and also denotes musical tones (e.g. A4 $=440 \mathrm{~Hz}$ ), whereas the linear horizontal axis shows in the insertion angle (degrees) of the electrode array. The two black lines represent the Stakhovskaya reference line and the Cochlear reference line. The averaged pitch matches for CI422 (blue) and CI512 (orange) are presented, split for experiments Test (solid line) and Re-test (dashed line). For the details of the coordinates of the two reference lines, see Supplemental Material 1. As expected by the tonotopic organization of the cochlea and the programming of the CI, we observed that the frequency of the pitch matches decreased with increasing insertion angle (i.e. towards the apex of the cochlea). Again, we can observe that the insertion of the CI512 electrode array was shallower than the insertion of the CI422 electrode array. For both groups, there was a significant difference ("mismatch") between the averaged data points per electrode contact (Test and Re-test experiments) and the Stakhovskaya reference line (two-sided one sample t-test, $p<0.001$; for complete statistics, see Supplemental Material 2). Compared to the Cochlear reference line, the CI422 group differs statistically significantly on all electrode contacts $(p<0.005)$, whereas the difference for the CI512 group was not statistically significant for E15 and E19 (at insertion angles of 230.17 and $284.56^{\circ}$, respectively; $p>0.05$ ). Importantly, the average mismatch of the CI512 group was smaller than the average mismatch of the CI422 group. 
Figure 3. Average pitch match versus insertion angle per group

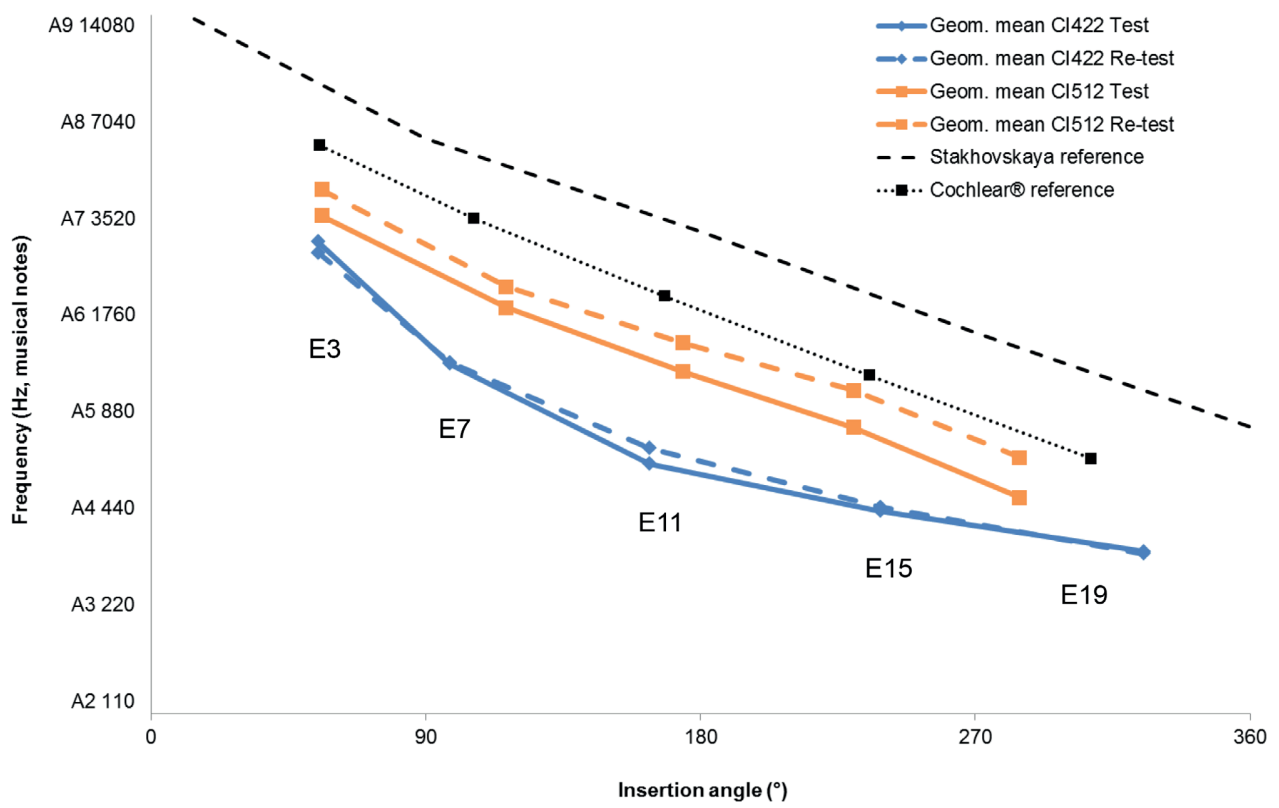

Legend: The averaged pitch matches (geometric mean, in $\mathrm{Hz}$ and musical notes, e.g. A4 = 440 Hz) per group (CI422 in blue, CI512 in orange) and per experiment (Test is solid line, Re-test is dashed line) and per electrode (E3, E7, E11, E15, E19) are plotted against the insertion angle (degrees). Also plotted are the Stakhovskaya reference line (striped black line) and the Cochlear reference line (dotted black line). For the x-coordinates of the Cochlear reference line, we used the averaged insertion angles per electrode contact for all patients. For readability of this figure, standard deviations are not included here. These can be observed in Figure 4.

\section{Mismatch}

To easily compare the two groups per electrode contact, we expressed the mismatch in octaves compared to the Stakhovskaya reference line (by definition 0 on y-axis in Figure 4; data relative to Cochlear reference not shown). We calculated the average mismatch relative to the references for all electrode contacts (E3, E7, E11, E15, and E19) for both experiments (Test and Re-test) per group. The average mismatch relative to the Stakhovskaya reference was -2.2 octave for the CI422 group, and -1.3 octave for the CI512 group (relative to the Cochlear reference: -1.4 octave and -0.5 , respectively). 
Figure 4. Mismatch expressed per electrode contact per group and experiment.

\section{Electrode}

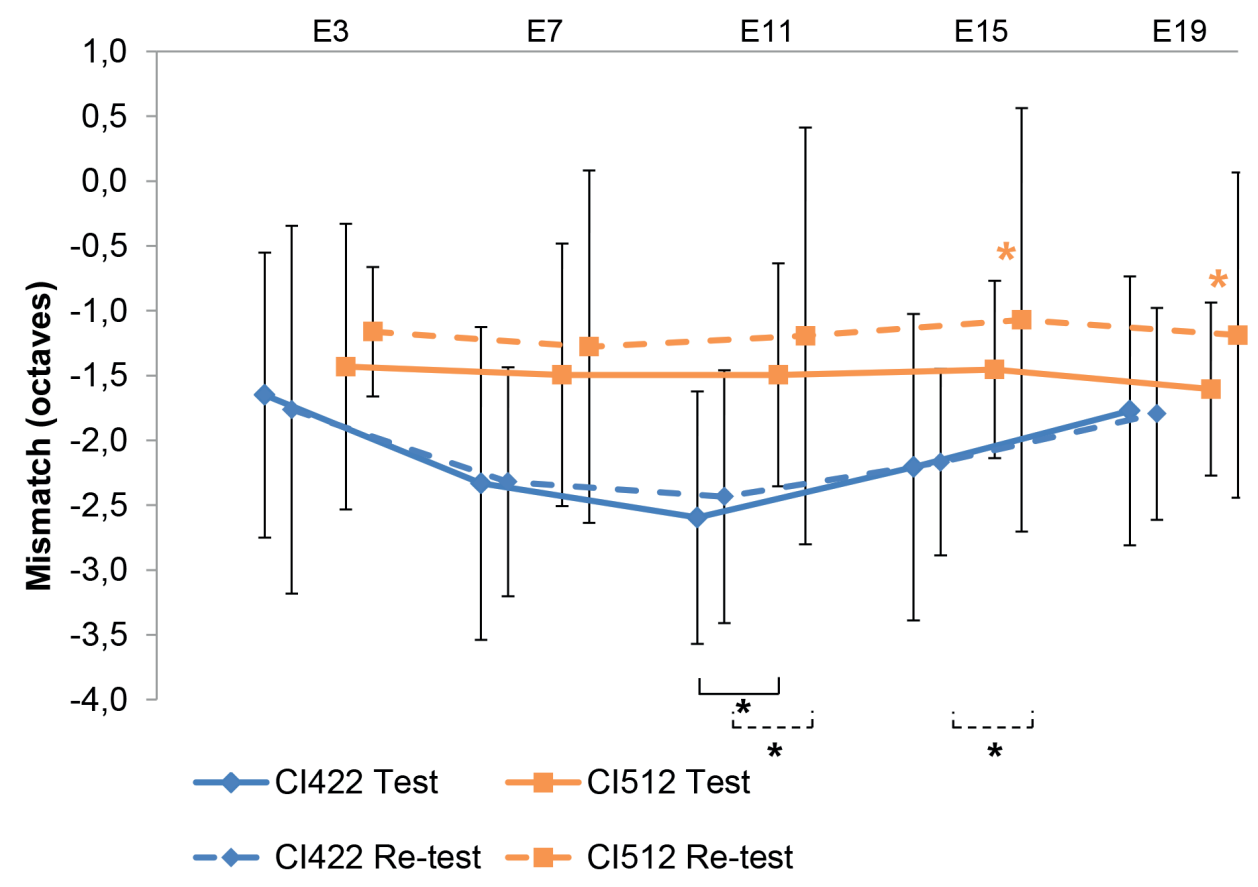

Legend: The mismatch relative to the Stakhovskaya reference is plotted per electrode (data relative to Cochlear reference not shown). Data points are displayed separately for the CI422 and CI512 groups, as well as for the Test and Re-test experiments. For the CI422 group, there is no significant difference between Test and Re-test for any of the electrodes. For the CI512 group, E15 and E19 differ significantly between Test and Re-test, indicated with orange asterisks. For E11, there is a significant difference between the two groups for both Test and Re-test, indicated with black asterisks. For E15, the difference between the two groups is statistically significant for Re-test, but not statistically significant for Test.

\section{Within-group comparisons}

For the CI422 group, there was neither a significant difference between Test and Re-test experiments for all electrode contacts together (Wilcoxon, $p=0.900$ ), nor for any of the individual electrode contacts ( $p>0.208)$. For the CI512 group, there was a significant difference between the Test and Re-test experiments ( $p=0.003$ ). Looking at specific electrode contacts, E3, E7, and E11 did not differ significantly between Test and Re-test, but E15 and E19 differed significantly between the two experiments $(p<0.05)$, indicated with orange asterisks in Figure 4. 


\section{Between-group comparisons}

For all electrode contacts together, there was a significant difference between the mismatches of the two groups (Mann-Whitney $\mathrm{U}$ test, $p<0.005$ ). When taking individual electrode contacts into account, there was no statistically significant difference between the groups for Test or Re-test for E3, E7, and E19 ( $p>0.14)$. For E11, there is a significant difference between the two groups for both Test and Re-test $(p<0.05)$. For E15, the difference between the two groups is statistically significant for Re-test $(p=0.013)$, but not for Test $(p=0.108)$, indicated with black asterisks in Figure 4.

\section{Correlations}

Due to the limited amount of data, correlations between patient characteristics and observed mismatch are of limited meaning, but our curiosity prevailed. We averaged the mismatch for all electrode contacts and for Test and Re-test experiments per patient. There were no correlations between the observed mismatch and patient characteristics age, duration of deafness, and experience with CI (maximum $\mathrm{R}^{2}: 0.191, p>0.05$ ). There was a significant correlation between the observed mismatch and $\mathrm{PTA}_{\text {be }}$ for the CI512 group $\left(\mathrm{R}^{2}\right.$ : 0.8259 , $p=0.012)$ as well as for the CI422 group $\left(\mathrm{R}^{2}: 0.6342, p<0.05\right)$ (Figure 5a). In this analysis we excluded outlier Pt 8 (green data point in Figure 5a). For all patients (including Pt 8), the effect was also significant $\left(\mathrm{R}^{2}: 0.3560, p=0.024\right)$. 
Figure 5a. Mismatch versus Pure Tone Average of the better ear

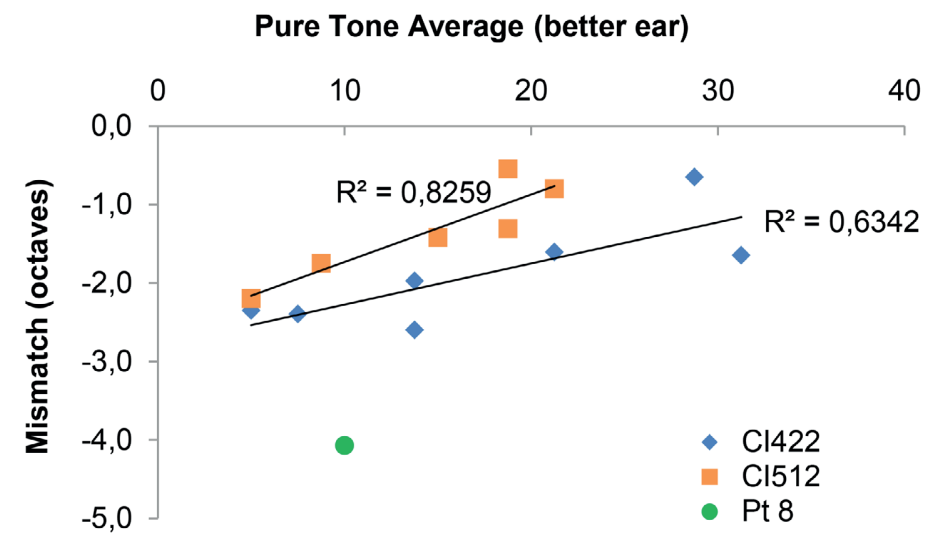

Legend: Mismatch (octaves) versus pure tone average of the better ear $\left(\mathrm{PTA}_{\mathrm{be}}\right)(\mathrm{dB} \mathrm{HL})$. There was a significant correlation between the observed mismatch and PTAbe for the CI512 group as well as for the CI422 group. Patient 8 (Pt 8, green dot) was excluded from this analysis. For all patients, the effect was also significant.

For both groups, there was no significant correlation between mismatch and the CVC phoneme recognition score (CI422 group, $\mathrm{R}^{2}: 0.1709, p>0.05$; CI512 group, $\mathrm{R}^{2}: 0.2248$, $p>0.05$ ) (Figure 5b; Pt 8 (green data point) was excluded again). For all patients including Pt 8 , there was no significant correlation either $\left(R^{2}: 0.2737, p>0.05\right)$. For complete statistical analyses, see Supplemental Material 2. 
Figure 5b. Mismatch versus CVC phoneme score.

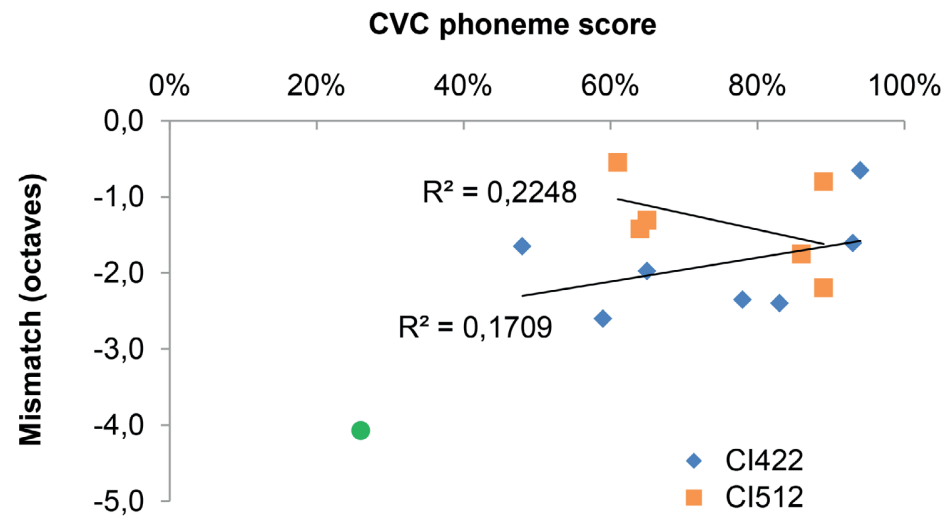

Legend: Mismatch (octaves) versus the score on a consonant-vowel-consonant (CVC) phoneme recognition task, 3 months after CI activation for the CI ear only. For both groups, there was no significant correlation between mismatch and the CVC phoneme score. Pt 8 (green dot) was excluded from this analysis. For all patients, the effect was also not significant.

\section{DISCUSSION}

In this study, we compared the results of electric-acoustic pitch match experiments of patients with a CI422 and a lateral wall electrode array and patients with a CI512 and a perimodiolar electrode array. Because of its shorter length, the CI512 electrode array was inserted shallower than the longer CI422 electrode array, but it was positioned closer to the modiolus axis due to its preformed curvature. We found average mismatches relative to the Stakhovskaya reference of -2.2 octave for the CI422 group and -1.3 octave for the CI512 group (relative to the Cochlear reference: -1.4 octave and -0.5 octave, respectively). For any given electrode contact, the mismatch to the two references was smaller for the CI512 electrode array than for the CI422 electrode array. For all electrode contacts together, there was a significant difference between the mismatches of the two groups. Results remained stable over time, with no significant difference between Test and Re-test experiments considering all electrode contacts. Neither group showed a significant correlation between the mismatch and CVC phoneme recognition score. 


\section{Observed mismatch}

Like in our previous report, the task appeared to be hard for the patients. ${ }^{14}$ They had difficulty matching the pitch of the acoustic stimulus to the pitch of the electric stimulus, partly because the stimuli sounded different. The difficulty of the task may have contributed to the observed large intra- and intersubject variability (see Supplementary Material 1). Although pitch-matching and pitch-fusion experiments involve different tasks, it is conceivable that the outcome variability observed in this study is related to the earlier reported finding that patients can experience broad binaural pitch fusion between the CI ear and the better ear, equal to bilateral CI users, bilateral HA users and bimodal CI users. ${ }^{30-32}$ In spite of the task difficulty, the average results were stable over the observed time (average interval between Test and Re-test experiments was 4.3 months) for both groups. The good reproducibility is in line with the only other report investigating electric-acoustic pitch matches over time. ${ }^{2}$

The average mismatch to the Stakhovskaya reference line was -2.2 octave for the CI422 group and -1.3 octave for the CI512 group. The difference in mismatch may partly be attributed to the distance to the modiolus, which is, for any given electrode (Figure 2a), shorter for a perimodiolar electrode array compared to a lateral wall electrode array. The range of observed mismatches is in line with our previous report (-2.0 octaves for ten SSD patients with CI422 $)^{14}$, and also concordant with other series evaluating various CI types in SSD patients..$^{2,4,7-9}$ There are several other explanations proposed for the observed mismatch, including methodological shortcomings in the pitch matching procedures ${ }^{33}$, tonotopical reorganization after $\mathrm{CI}$ activation ${ }^{2,10}$, the electrical current between the electrode contacts and the extracochlear reference electrode following the trajectory of least resistance which may differ from the histologically observed radial fiber trajectories ${ }^{5,14}$, and finally the degree of contralateral residual hearing ${ }^{11}$. One paper observed no mismatch between their electricacoustic pitch match comparisons and the Stakhovskaya reference line. ${ }^{34}$ They only used data that remained after so-called "sanity checks" of the experiments: presumably unreliable pitch match data were discarded. As we pointed out earlier in this paragraph, and in our previous 
paper $^{14}$, we observed a large intra- and intersubject variability. We feel it is important to report all measurements and thereby present this variability, and chose not discard any data.

Remember that the audiograms of the two groups were similar (Figure 1), and thus hearing threshold differences in the better ear cannot explain the difference in mismatch between the two groups. Psychoacoustic experiments in patients with asymmetric hearing loss showed that high-frequency hearing loss distorts pitch coding for pure tones with a frequency in the hearing loss range, resulting in pathologic hearing (diplacusis). ${ }^{13}$ In normalhearing subjects, the pitch of a pure tone may differ slightly between ears ${ }^{31}$, but a fused single pitch is perceived in bilateral stimulation. Then, in the frequency range of the hearing loss, the pitch perceived in the poor ear is lower than the pitch perceived in the better ear, and becomes lower as the hearing loss increases. Translated to our experiment, the pitch perceived in the better ear is lower when the hearing loss is bigger and concomitantly the mismatch will decrease, while the CI-evoked pitch for a specific electrode contact is constant. This could explain the correlation we detected between the mismatch and PTA $_{\text {be }}$ (Figure 5a): with increasing hearing loss, the mismatch becomes smaller. We investigated a group of SSD patients with mild to moderate (mainly high-frequency) hearing loss in the better ear; when comparing our mismatch results to the results of groups with severe to profound (highfrequency) hearing loss ${ }^{2,4,7-9}$, the found mismatch was about equal. Apparently, the effects of distorted pitch perception due to high-frequency hearing loss are small compared to those of the, still unknown, cause(s) of the mismatch.

\section{Intracochlear electrode array position}

For both groups, we observed a large variance between maximum insertion angles of the most apical electrode contact (deepest and shallowest insertion of E22 in CI422 group: 537 and $235^{\circ}$ (median $399.7^{\circ}$ ), whereas in CI512 group: $421^{\circ}$ and $242^{\circ}\left(\right.$ median $\left.307.3^{\circ}\right)$. The large variation in insertion depth was also reported by Landsberger et al. ${ }^{6}$ In contrast, they found a median insertion angle of the most apical electrode contact of the Contour Advance electrode 
array of $375^{\circ}$, which was deeper than the median insertion angle of $307.3^{\circ}$ in our study.

Some colleagues stated that deeper insertion leads to improved speech perception ${ }^{1,35}$, whereas others disagree. ${ }^{36} \mathrm{~A}$ similar debate continues about the position of the electrode array: some argue that a lateral wall electrode array led to better performance ${ }^{18}$, whereas others found that a perimodiolar position of the electrode array was correlated with better performance. ${ }^{19-21}$

\section{Methodological considerations}

We presented the first series in which we investigated electric-acoustic pitch matches between two electrode array types in patients with SSD. Our patients have good hearing in their better ear, not resulting in a distorted pitch perception. ${ }^{13}$ Furthermore, we determined the insertion angle based on HRCT scans by a semiautomatic method, with which we could also determine radius and elevation of the electrode contacts. ${ }^{15}$

There are also limitations of our approach. First, we did not match the loudness at all acoustic frequencies that we tested. This would have resulted in too much patient effort and thus compromise the willingness of patients to participate. Consequently, in our current results the pitch matches may have been influenced by (mainly high-frequency) hearing loss in the better ear. However, we know that the influence of loudness on pitch perception is negligible in the tested range. ${ }^{23}$ Second, there was a small sample size (CI422 group: $\mathrm{n}=$ 8, CI512 group: $\mathrm{n}=6$ ). Third, the intracochlear position of the electrode array in Pt 8 was very shallow, resulting in 4-5 disabled basal electrode contacts. Consequently, we did not consider E3 in this patient. Fourth, our semiautomatic method to determine intracochlear electrode array position did not allow us to determine if a scalar shift of the electrode array had occurred, which may also be of influence on performance and possibly mismatch. ${ }^{37}$ Finally, we tested performance using a CVC phoneme recognition score only. Since this is not a test for binaural hearing, other tests might be required to determine performance in this population. 


\section{Implications of findings}

In our previous report, we argued that the results of pitch matching experiments should be interpreted with caution: observed mismatches should not necessarily lead to adaptation of the default frequency allocation settings, since we did not observe a correlation between mismatch and performance. ${ }^{14}$ This conclusion was in contrast with evidence pointing in the direction that it is important to deliver auditory information at the correct cochlear place. ${ }^{35,38,39}$ Possibly, reorganization of the neural system to the new frequency-place situation may result in little impact of mismatch on performance. ${ }^{40}$ In our present study, we found that the pitch mismatch was smaller for the perimodiolar electrode array than for the lateral wall electrode array. However, our study is limited by a small number of patients per group. Therefore, we cannot draw conclusions regarding implications for clinicians and patients.

\section{CONCLUSION}

We found average mismatches relative to the Stakhovskaya reference of -2.2 octave for the CI422 group and -1.3 octave for the CI512 group (relative to the Cochlear reference: -1.4 octave and -0.5 octave, respectively). For any given electrode contact, the mismatch to the two references was smaller for the CI512 electrode array than for the CI422 electrode array. For all electrode contacts together, there was a significant difference between the mismatches of the two groups. Results remained stable over time (average interval between Test and Re-test experiments: 4.3 months), with no significant difference between Test and Re-test experiments considering all electrode contacts. Neither group showed a significant correlation between the mismatch and CVC phoneme recognition score. 


\section{Conflict of Interest}

The authors have no conflicts of interest to declare.

\section{Funding}

This study was partly funded by Cochlear Ltd. as an unrestricted research grant. By research contract, Cochlear Ltd. did not have influence on the study design, data collection, analysis, data interpretation, and publication.

\section{SUPPLEMENTARY MATERIAL}

The supplementary material can be accessed online via www.karger.com/doi/10.1159/000499154

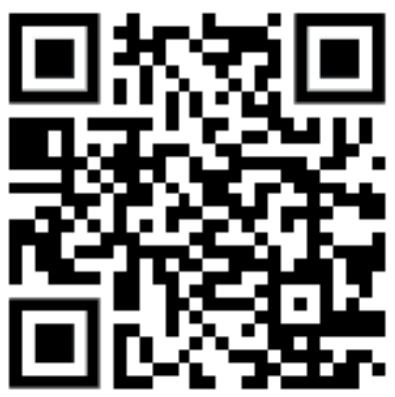

Supplemental Material 1 Individual patient data

Supplemental Material 2 Statistics 


\section{REFERENCES}

1. Buchman CA, Dillon MT, King ER, Adunka MC, Adunka OF, Pillsbury HC. Influence of cochlear implant insertion depth on performance: a prospective randomized trial. Otol Neurotol 2014;35(10):1773-1779.

2. Vermeire K, Landsberger DM, Van de Heyning PH, Voormolen M, Kleine Punte A, Schatzer R, Zierhofer C. Frequency-place map for electrical stimulation in cochlear implants: Change over time. Hear Res 2015;326:8-14.

3. Greenwood DD. A cochlear frequency-position function for several species--29 years later. J Acoust Soc Am 1990;87(6):2592-2605.

4. Dorman MF, Spahr T, Gifford R, Loiselle L, McKarns S, Holden T, Skinner M, Finley C. An electric frequency-to-place map for a cochlear implant patient with hearing in the nonimplanted ear. J Assoc Res Otolaryngol 2007;8(2):234-240.

5. Stakhovskaya O, Sridhar D, Bonham BH, Leake PA. Frequency map for the human cochlear spiral ganglion: implications for cochlear implants. J Assoc Res Otolaryngol 2007;8(2):220-233.

6. Landsberger DM, Svrakic M, Roland JT Jr, Svirsky M. The Relationship Between Insertion Angles, Default Frequency Allocations, and Spiral Ganglion Place Pitch in Cochlear Implants. Ear Hear 2015;36(5):e207-213.

7. Boëx C, Baud L, Cosendai G, Sigrist A, Kós MI, Pelizzone M. Acoustic to electric pitch comparisons in cochlear implant subjects with residual hearing. J Assoc Res Otolaryngol 2006;7(2):110-124.

8. Baumann U, Nobbe A. The cochlear implant electrode-pitch function. Hear Res 2006;213(1-2):34-42.

9. Schatzer R, Vermeire K, Visser D, Krenmayr A, Kals M, Voormolen M, Van de Heyning P, Zierhofer C. Electric-acoustic pitch comparisons in single-sided-deaf cochlear implant users: frequency-place functions and rate pitch. Hear Res 2014;309:26-35.

10. McDermott H, Sucher C, Simpson A. Electro-acoustic stimulation. Acoustic and electric 
pitch comparisons. Audiol Neurotol 2009;14(Suppl 1):2-7.

11. Vermeire K, Nobbe A, Schleich P, Nopp P, Voormolen MH, Van de Heyning PH. Neural tonotopy in cochlear implants: an evaluation in unilateral cochlear implant patients with unilateral deafness and tinnitus. Hear Res 2008;245(1-2):98-106.

12. Xu J, Xu SA, Cohen LT, Clark GM. Cochlear view: postoperative radiography for cochlear implantation. Am J Otol 2000;21:49-56.

13. Gaeth JH, Norris TW. Diplacusis in unilateral high-frequency hearing losses. J Speech Hear Res 1965;8:63-75.

14. Peters JPM, Bennink E, Grolman W, Van Zanten GA. Electro-acoustic pitch matching experiments in patients with single-sided deafness and a cochlear implant: Is there a need for adjustment of the default frequency allocation tables? Hear Res 2016;342:124133.

15. Bennink E, Peters JPM, Wendrich AW, Vonken EJ, Van Zanten GA, Viergever MA. Automatic Localization of Cochlear Implant Electrode Contacts in CT. Ear Hear 2017;38(6):e376-e384.

16. Von Wallenberg E, Briggs R. Cochlear's unique electrode portfolio now and in the future. Cochlear Implants Int 2014;15(Suppl 1):S59-61.

17. Gibson P, Boyd P. Optimal electrode design: Straight versus perimodiolar. Eur Ann Otorhinolaryngol Head Neck Dis 2016;133(Suppl 1):S63-65.

18. O’Connell BP, Hunter JB, Gifford RH, Rivas A, Haynes DS, Noble JH, Wanna GB. Electrode Location and Audiologic Performance After Cochlear Implantation: A Comparative Study Between Nucleus CI422 and CI512 Electrode Arrays. Otol Neurotol 2016;37(8):1032-1035.

19. Holden LK, Finley CC, Firszt JB, Holden TA, Brenner C, Potts LG, Gotter BD, Vanderhoof SS, Mispagel K, Heydebrand G, Skinner MW. Factors affecting open-set word recognition in adults with cochlear implants. Ear Hear 2013;34(3):342-360.

20. Esquia Medina GN, Borel S, Nguyen Y, Ambert-Dahan E, Ferrary E, Sterkers O, Grayeli 
AB. Is electrode-modiolus distance a prognostic factor for hearing performances after cochlear implant surgery? Audiol Neurotol 2013;18(6):406-413.

21. Van der Beek FB, Boermans PP, Verbist BM, Briaire JJ, Frijns JHM. Clinical evaluation of the Clarion CII HiFocus 1 with and without positioner. Ear Hear 2005;26(6):577-592.

22. Peters JPM, Van Zon A, Smit AL, Van Zanten GA, De Wit GA, Stegeman I, Grolman W. CINGLE-trial: Cochlear Implantation for siNGLE-sided deafness, a Randomized controlled trial and economic evaluation. BMC Ear, Nose and Throat Disorders 2015;15:3.

23. Strange P. The sense of pitch and local increase in threshold. J Physiol 1955;129(2):225236.

24. Verbist BM, Joemai RMS, Briaire JJ, Teeuwisse WM, Veldkamp WJH, Frijns JHM. Cochlear coordinates in regard to cochlear implantation: a clinically individually applicable 3 dimensional CT-based method. Otol Neurotol 2010;31(5):738-744.

25. Verbist BM, Skinner MW, Cohen LT, Leake PA, James C, Boëx C, Holden TA, Finley CC, Roland PS, Roland JT Jr, Haller M, Patrick JF, Jolly CN, Faltys MA, Briaire JJ, Frijns JHM. Consensus panel on a cochlear coordinate system applicable in histologic, physiologic, and radiologic studies of the human cochlea. Otol Neurotol 2010;31(5):722-730.

26. Erixon E, Rask-Andersen H. How to predict cochlear length before cochlear implantation surgery. Acta Otolaryngol 2013;133(12):1258-1265.

27. Van der Marel KS, Briaire JJ, Wolterbeek R, Snel-Bongers J, Verbist BM, Frijns JHM. Diversity in cochlear morphology and its influence on cochlear implant electrode position. Ear Hear 2014;35(1):e9-20.

28. Bosman AJ, Smoorenburg GF. Intelligibility of Dutch CVC syllables and sentences for listeners with normal hearing and with three types of hearing impairment. Audiology $1995 ; 34(5): 260-284$.

29. Rader T, Döge J, Adel Y, Weissgerber T, Baumann U. Place dependent stimulation rates improve pitch perception in cochlear implantees with single-sided deafness. Hear Res 2016;339:94-103.

30. Reiss LA, Ito RA, Eggleston JL, Wozny DR. Abnormal binaural spectral integration in 
cochlear implant users. J Assoc Res Otolaryngol 2014;15:235-248.

31. Reiss LA, Shayman CS, Walker EP, Bennet KO, Fowler JR, Hartling CL, Glickman B, Lasarev MR, Oh Y. Binaural pitch fusion: Comparison of normal-hearing and hearingimpaired listeners. J Acoust Soc Am 2017;141:1909-1920.

32. Reiss LA, Fowler JR, Hartling CL, Oh Y. Binaural Pitch Fusion in Bilateral Cochlear Implant Users. Ear Hear 2018;39(2):390-397.

33. Devocht EM, Dees G, Arts RA, Smits JJ, George EL, van Hoof M, Stokroos RJ. Revisiting Place-Pitch Match in CI Recipients Using 3D Imaging Analysis. Ann Otol Rhinol Laryngol 2016;125(5):378-384.

34. Carlyon RP, Macherey O, Frijns JH, Axon PR, Kalkman RK, Boyle P, Baguley DM, Briggs J, Deeks JM, Briaire JJ, Barreau X, Dauman R. Pitch comparisons between electrical stimulation of a cochlear implant and acoustic stimuli presented to a normal-hearing contralateral ear. J Assoc Res Otolaryngol 2010;11(4):625-640.

35. Zhou X, Li H, Galvin JJ 3rd, Fu QJ, Yuan W. Effects of insertion depth on spatial speech perception in noise for simulations of cochlear implants and single-sided deafness. Int J Audiol 2016;1:1-8.

36. Van der Marel KS, Briaire JJ, Verbist BM, Muurling TJ, Frijns JHM. The influence of cochlear implant electrode position on performance. Audiol Neurotol 2015;20(3):202211.

37. Shaul C, Dragovic AS, Stringer AK, O'Leary SJ, Briggs RJ. Scalar localisation of perimodiolar electrodes and speech perception outcomes. J Laryngol Otol 2018;(11):10001006.

38. Oxenham AJ, Bernstein JG, Penagos H. Correct tonotopic representation is necessary for complex pitch perception. Proc Natl Acad Sci USA 2004;101(5):1421-1425.

39. Reiss LA, Eggleston JL, Walker EP, Oh Y. Two Ears Are Not Always Better than One: Mandatory Vowel Fusion Across Spectrally Mismatched Ears in Hearing-Impaired Listeners. J Assoc Res Otolaryngol 2016;17(4):341-356.

40. Eggermont JJ. Acquired hearing loss and brain plasticity. Hear Res 2017;343:176-190. 


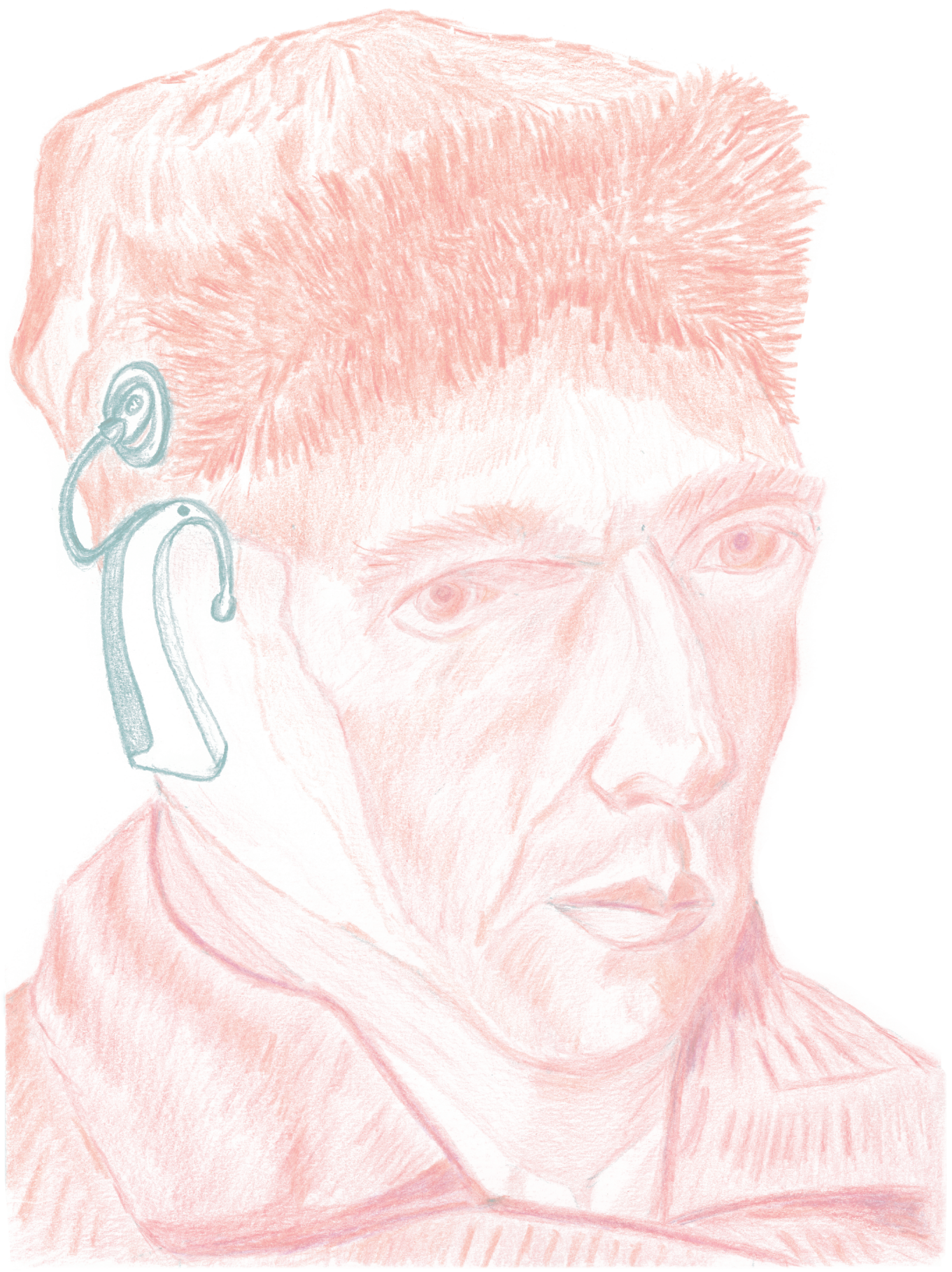




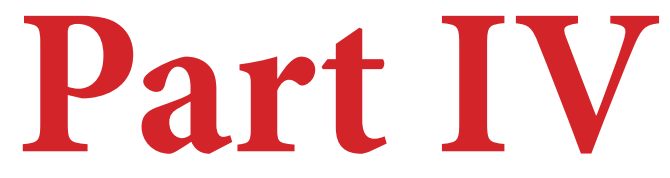

Quality of reporting 


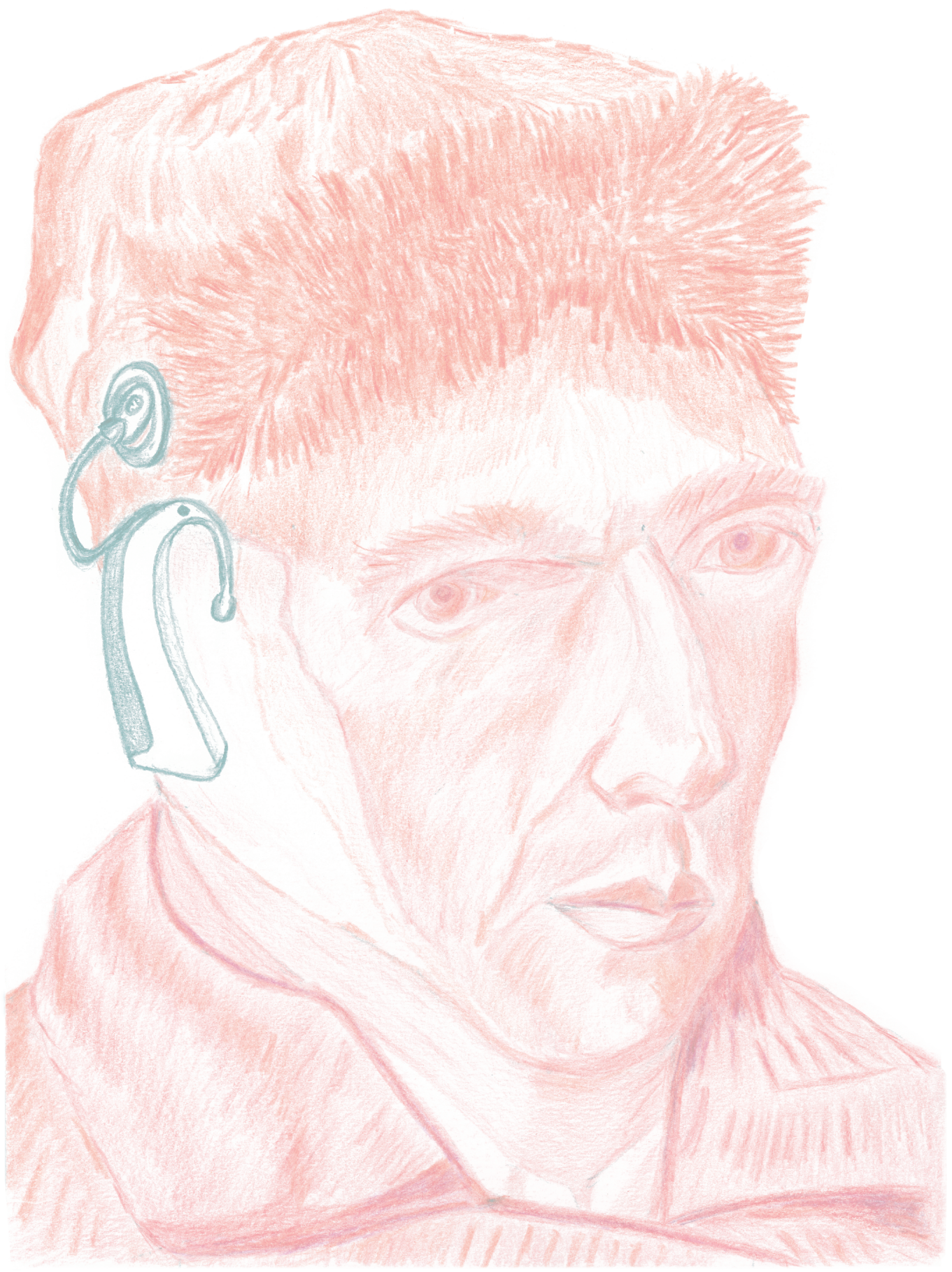




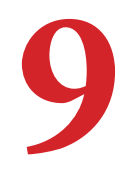

\section{Reporting Quality of Systematic Reviews and Meta-Analyses of Otorhinolaryngologic Articles Based on the PRISMA Statement}




\section{ABSTRACT}

\section{Background}

Systematic reviews (SRs) and meta-analyses (MAs) provide the highest possible level of evidence. However, poor conduct or reporting of SRs and MAs may reduce their utility. The PRISMA Statement (Preferred Reporting Items for Systematic reviews and Meta-Analyses) was developed to help authors report their SRs and MAs adequately.

\section{Objectives}

Our objectives were to (1) evaluate the quality of reporting of SRs and MAs and their abstracts in otorhinolaryngologic literature using the PRISMA and PRISMA for Abstracts checklists, respectively, (2) compare the quality of reporting of SRs and MAs published in Ear Nose Throat (ENT) journals to the quality of SRs and MAs published in the 'gold standard' Cochrane Database of Systematic Reviews (CDSR), and (3) formulate recommendations to improve reporting of SRs and MAs in ENT journals.

\section{Methods}

On September 3, 2014, we searched the Pubmed database using a combination of filters to retrieve SRs and MAs on otorhinolaryngologic topics published in 2012 and 2013 in the top 5 ENT journals (ISI Web of Knowledge 2013) or CDSR and relevant articles were selected. We assessed how many, and which, PRISMA (for Abstracts) items were reported adequately per journal type.

\section{Results}

We identified large differences in the reporting of individual items between the two journal types with room for improvement. In general, SRs and MAs published in ENT journals (n $=31$ ) reported a median of $54.4 \%$ of the PRISMA items adequately, whereas the 49 articles published in the CDSR reported a median of $100.0 \%$ adequately (difference statistically 
significant, $p<0.001)$. For abstracts, medians of $41.7 \%$ for ENT journals and $75.0 \%$ for the CDSR were found $(p<0.001)$.

\section{Conclusion}

The reporting of SRs and MAs in ENT journals leaves room for improvement and would benefit if the PRISMA Statement were endorsed by these journals. 


\section{INTRODUCTION}

Systematic reviews (SRs) and meta-analyses (MAs) have the highest possible level of evidence in medical literature. ${ }^{1}$ Both SRs and MAs combine the results of a number of trials. Poorly conducted primary trials, even randomised controlled trials (RCTs), could lead to the introduction of bias (systematic inclination inhibiting impartial judgment). Bias may reduce the utility of SRs and MAs. Clear presentation of what was planned, done and found in SRs and MAs is essential to value its findings, because clinicians use the results directly in clinical care.

In 1999 the QUORUM Statement (QUality Of Reporting Of Meta-analyses) was developed to help authors report meta-analyses adequately. ${ }^{2}$ In 2009, the QUORUM Statement was replaced by the PRISMA Statement (Preferred Reporting Items for Systematic reviews and Meta-Analyses) to also apply to SRs. ${ }^{3,4}$ Adherence to the QUORUM ${ }^{5}$ or PRISMA ${ }^{6-10}$ Statements improved the quality of reporting of published SRs and MAs.

To our knowledge, the quality of reporting of SRs and MAs has not been assessed in otorhinolaryngologic literature yet. In current medicine, where evidence-based medicine is taking a prominent place, adequate reporting of the findings of SRs and MAs is important, also for clinical practice and patient care. Therefore, our primary aim was to assess the quality of reporting of (abstracts of) SRs and MAs in otorhinolaryngologic literature. Our second aim was to compare the quality of reporting of SRs and MAs published in Ear Nose Throat (ENT) journals to otorhinolaryngologic SRs and MAs published in the Cochrane Database of Systematic Reviews (CDSR). Finally, we formulated recommendations to improve the reporting of SRs and MAs in otorhinolaryngology. 


\section{METHODS}

\section{Journals}

We included SRs from the top 5 ENT journals, based on ISI Web of Knowledge 2013 impact factors (www.webofknowledgde.com). The top 5 ENT journals are Head \& Neck (Head Neck), Hearing Research (Hear Res), Ear \& Hearing (Ear Hear), Rhinology and Journal of the Association for Research in Otolaryngology (JARO) (Table 1). None of these top 5 ENT journals endorse the PRISMA Statement in their instructions to authors (evaluated on November $\left.6^{\text {th }}, 2014\right)$. To compare the quality of reporting of SRs and MAs published in ENT journals to the 'gold standard' of systematic reviews of the literature, SRs and MAs published in the CDSR were extracted. The CDSR does endorse the PRISMA Statement.

Table 1. Impact Factors (2013) of the Top 5 Ear Nose Throat (ENT) journals and Cochrane Database of Systematic Reviews

\begin{tabular}{llr}
\hline Journal & & Impact Factor $^{*}$ \\
\hline ENT journals (journal abbreviation) & 1. Head \& Neck (Head Neck) & 3.006 \\
& 2. Hearing Research (Hear Res) & 2.848 \\
& 3. Ear \& Hearing (Ear Hear) & 2.833 \\
& 4. Rhinology & 2.779 \\
& 5. Journal of the Association for Research in & 2.547 \\
& Otolaryngology (JARO) &
\end{tabular}

Cochrane Database of Systematic Reviews (CDSR)

\section{Legend:}

* Source: ISI Web of Knowledge 2013, Journal Citations Reports via www.webofknowledge.com, accessed on September 3rd, 2014.

\section{Search (date of search: 3 September 2014)}

We performed a Pubmed search using five filters. First, an adapted version of the Cochrane ENT search filter was used to retrieve otorhinolaryngologic articles (Supporting Information 1). ${ }^{11}$ Second, to retrieve only SRs and MAs, the Pubmed filter for SRs and MAs was used. ${ }^{12}$ Third, a date restriction was applied to retrieve only articles indexed in 2012 and 2013. Fourth, 
a filter was used to search only in the top 5 ENT journals and a filter was used to search only in the CDSR. Fifth, editorials, letters, news and comments as publication type were excluded (for complete search: see Supporting Information 1). Finally, a combination was made with a search syntax for the ENT journals and the CDSR using Boolean operator AND.

\section{Study selection}

Two authors (JPMP and IS) independently assessed titles, abstracts and full texts of the retrieved articles to check if the study was indeed a SR or MAs and if it was conducted in the otorhinolaryngologic field. To be considered as a study in the otorhinolaryngologic field, studies must assess patient populations generally treated by otorhinolaryngologists or investigate a procedure generally performed by otorhinolaryngologists, including head and neck surgery (Supporting Information 1).

Throughout this paper, we adopt the definition of SRs and MAs of the PRISMA Statement ${ }^{3}$ and Cochrane Collaboration (http://handbook.cochrane.org): "a systematic review is a review of a clearly formulated question that uses systematic and explicit methods to identify, select, and critically appraise relevant research, and to collect and analyze data from the studies that are included in the review. Statistical methods (meta-analysis) may or may not be used to analyze and summarize the results of the included studies. Meta-analysis refers to the use of statistical techniques in a systematic review to integrate the results of included studies".

Discrepancies between the two reviewers were discussed until consensus was reached. When no consensus could be reached, an independent otorhinolaryngologist was consulted.

\section{PRISMA Statement adherence}

The PRISMA 2009 checklist was used to score the quality of reporting. ${ }^{3}$ The included articles were read and assessed independently by two authors (JPMP and IS). We evaluated the 
number of items of the PRISMA checklist that were adequately reported. The total number of items on the PRISMA checklist is 27, however, item 2 (Abstract) was scored separately (see PRISMA for Abstracts adherence). Some items of the PRISMA checklist (item 14, 16, 21, and 23) are specific for meta-analysis only. They were not scored as missing or inadequately reported when not applicable. A more detailed explanation on how items were assessed can be found in Supporting Information 2. Differences in opinion were discussed until consensus was reached.

\section{PRISMA for Abstracts adherence}

The abstracts of the included SRs and MAs were assessed using the PRISMA for Abstracts checklist. ${ }^{13}$ The PRISMA for Abstracts checklist is a detailed checklist of what items should be reported in abstracts of SRs and MAs. The total number of items on the PRISMA for Abstracts checklist is 12. A more detailed explanation on how all 12 items were assessed can be found in Supporting Information 3.

\section{$\underline{\text { Data analysis }}$}

We calculated descriptive statistics per item of the PRISMA checklist and the PRISMA for Abstracts checklist. Using the Chi-square test, we evaluated if there was a statistically significant difference between the two journal types in the reporting per item.

Furthermore, we divided the number of adequately reported PRISMA items by a possible total of 26 items (Item 2, Abstract, was scored separately) if the assessed paper was a MA and divided by a possible total of 22 items if the assessed paper was a SR, resulting in a percentage. The higher the percentage, the more adequately the SR or MA was reported. For the assessment of the abstracts, the number of adequately reported PRISMA for Abstracts items was divided by a possible total of 12 . Subsequently, we calculated the median and mean percentages (and 95\% confidence intervals (CI)) per journal type to be able to compare the reporting of specific PRISMA (for Abstracts) items between the SRs and MAs published in 
ENT journals and in the CDSR. The two-tailed Mann-Whitney U test for two independent samples was used to compare PRISMA (for Abstracts) scores for SRs and MAs published in ENT journals and in CDSR.

Statistical tests were performed using SPSS v20 statistics package. A $p$-value of $<$ 0.05 was considered statistically significant.

\section{RESULTS}

\section{$\underline{\text { Search }}$}

The search process is shown in Figure 1. The combined search syntaxes yielded 36 articles from ENT journals and 91 articles from the CDSR (Supporting Information 1).

Figure 1. Flowchart of search (date of search: 3 September 2014)

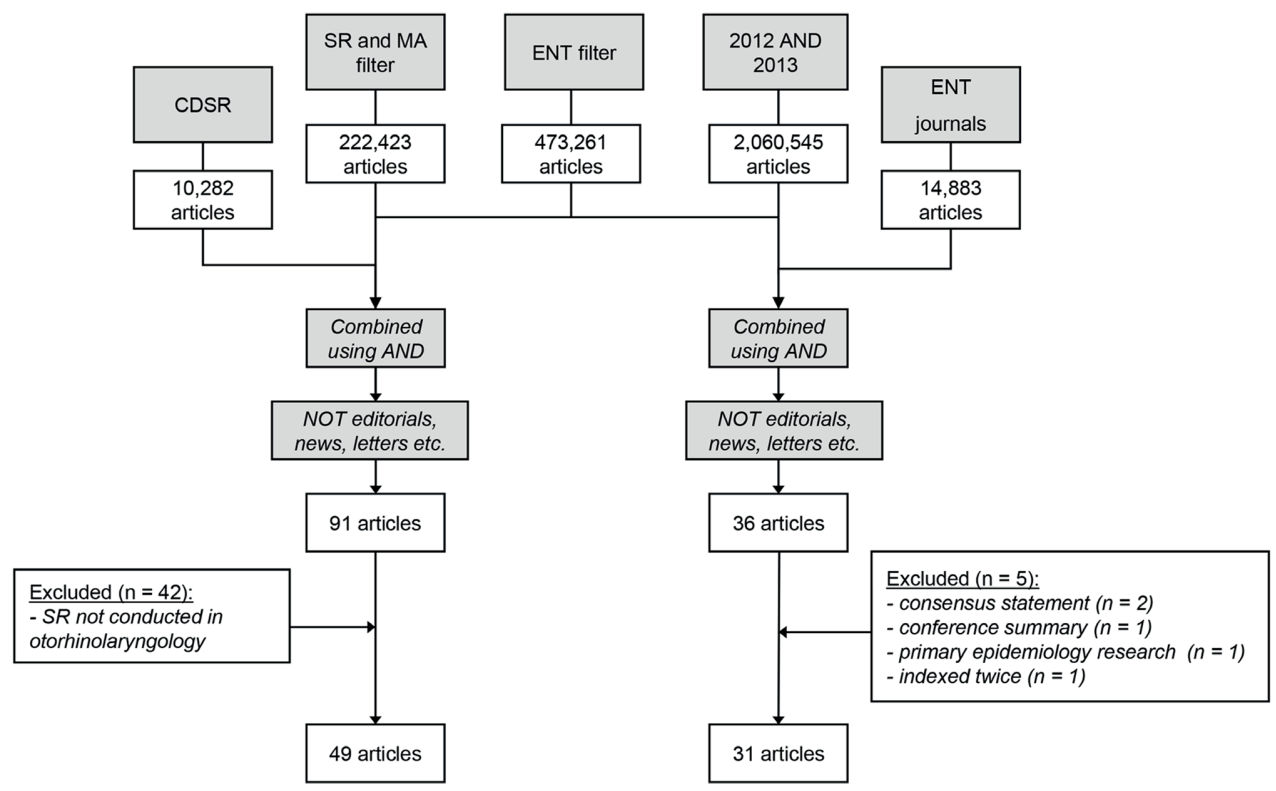

Legend: $\mathrm{SR}=$ Systematic Review, MA = meta-analysis, ENT $=$ Ear, Nose, Throat, CDSR = Cochrane Database of Systematic Reviews. 


\section{$\underline{\text { Study selection }}$}

All 36 articles published in ENT journals qualified as studies that were conducted in the otorhinolaryngologic research field. Not all retrieved articles were true SRs or MAs (i.e. the Pubmed filter is not 100\% specific for SRs and MAs): 2 papers were consensus statements, 1 paper was a summary of a conference, 1 paper was an epidemiologic paper including SRs and 1 paper was indexed twice; the latest published version of this article was included. The remaining 31 articles (Head Neck $n=24$, Hear Res $n=1$, Ear Hear $n=2$, Rhinology $n=4$, JARO $\mathrm{n}=0$ ) were further analysed, 26 were SRs and 5 were MAs.

All 91 articles published in CDSR were SRs or MAs. Of these, 42 did not qualify as studies conducted in the otorhinolaryngologic research field. The remaining 49 articles were included for data analysis. Of these, 10 were pure SRs and 39 also conducted a meta-analysis. These 49 SRs were conducted in large part by the Cochrane Ear, Nose and Throat Disorders group $(n=25)$, but also by the Acute Respiratory Infections group ( $\mathrm{n}=18)$, the Anaesthesia group $(n=2)$, the Childhood Cancer group, the Occupational Safety and Health group, the Developmental, Psychosocial and Learning Problems group, and the Cystic Fibrosis and Genetic Disorders group (each $n=1)$.

\section{$\underline{\text { PRISMA }}$}

The exact percentages of adequately reported PRISMA items for SRs and MAs published in ENT journals and CDSR are presented in Table 2, as well as the significance of the difference between the two journal types. A graphic illustration is provided in Figure 2. 


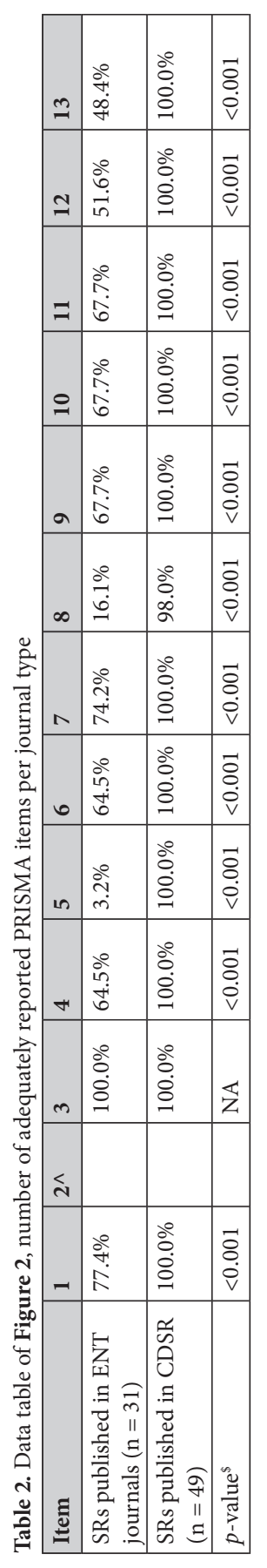

\begin{tabular}{|c|c|c|c|c|}
\hline ה & $\begin{array}{l}\stackrel{\circ}{\circ} \\
\underset{j}{\mathrm{~m}}\end{array}$ & 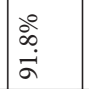 & $\mid \begin{array}{l}\overrightarrow{0} \\
\dot{\vec{v}}\end{array}$ & \multirow{15}{*}{ 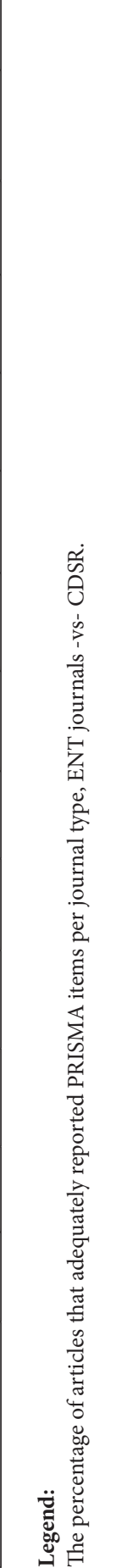 } \\
\hline ה: & 官 & 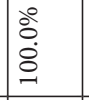 & \begin{tabular}{|l|}
$\vec{\Delta}$ \\
$\dot{\vec{v}}$ \\
\end{tabular} & \\
\hline in & $\begin{array}{l}\stackrel{\circ}{\circ} \\
\dot{\infty} \\
i n\end{array}$ & 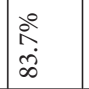 & 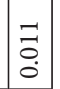 & \\
\hline I & 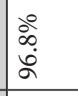 & 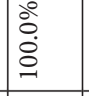 & \begin{tabular}{l} 
\\
\multirow{2}{*}{} \\
0 \\
\end{tabular} & \\
\hline 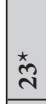 & 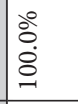 & 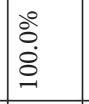 & $\vec{z}$ & \\
\hline ( & $\begin{array}{l}\text { भें } \\
\text { भे }\end{array}$ & 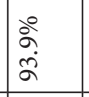 & \begin{tabular}{|l|}
$\overrightarrow{\vec{o}}$ \\
$\dot{\vec{v}}$ \\
$\dot{v}$
\end{tabular} & \\
\hline$\frac{\pi}{2}$ & 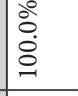 & 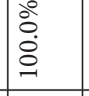 & $\mathbb{z}$ & \\
\hline กิ & $\begin{array}{l}\text { o̊ } \\
\text { in } \\
\end{array}$ & $\begin{array}{l}\text { के } \\
\text { گू }\end{array}$ & \begin{tabular}{|l|}
$\overrightarrow{8}$ \\
$\dot{\vec{\theta}}$ \\
\end{tabular} & \\
\hline 2 & 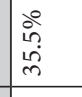 & 客 & \begin{tabular}{|l|}
$\vec{\Xi}$ \\
$\dot{\vec{v}}$ \\
$\mathrm{v}$
\end{tabular} & \\
\hline$\stackrel{\infty}{\circ}$ & 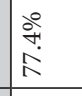 & 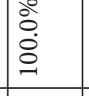 & \begin{tabular}{|l|}
$\overrightarrow{\grave{a}}$ \\
$\dot{\vec{v}}$
\end{tabular} & \\
\hline$\cong$ & $\begin{array}{l}\stackrel{0}{2} \\
\hat{1} \\
0\end{array}$ & 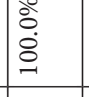 & \begin{tabular}{|l|}
$\overrightarrow{\grave{O}}$ \\
$\dot{\dot{\theta}}$
\end{tabular} & \\
\hline 屯。 & 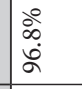 & 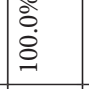 & 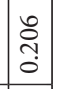 & \\
\hline 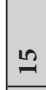 & $\begin{array}{l}\stackrel{\circ}{0} \\
\text { ì }\end{array}$ & $\begin{array}{l}\stackrel{\circ}{\circ} \\
\text { à }\end{array}$ & $\mid \begin{array}{l}\vec{\Delta} \\
\dot{\vec{v}}\end{array}$ & \\
\hline \pm & 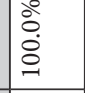 & $\begin{array}{l}0 \\
0 \\
\infty \\
\infty \\
\infty\end{array}$ & 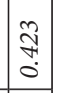 & \\
\hline Е & 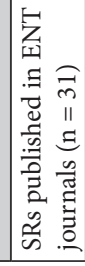 & 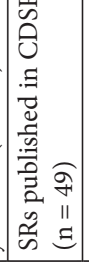 & 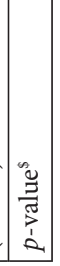 & \\
\hline
\end{tabular}


Figure 2. Number of adequately reported PRISMA items per journal type

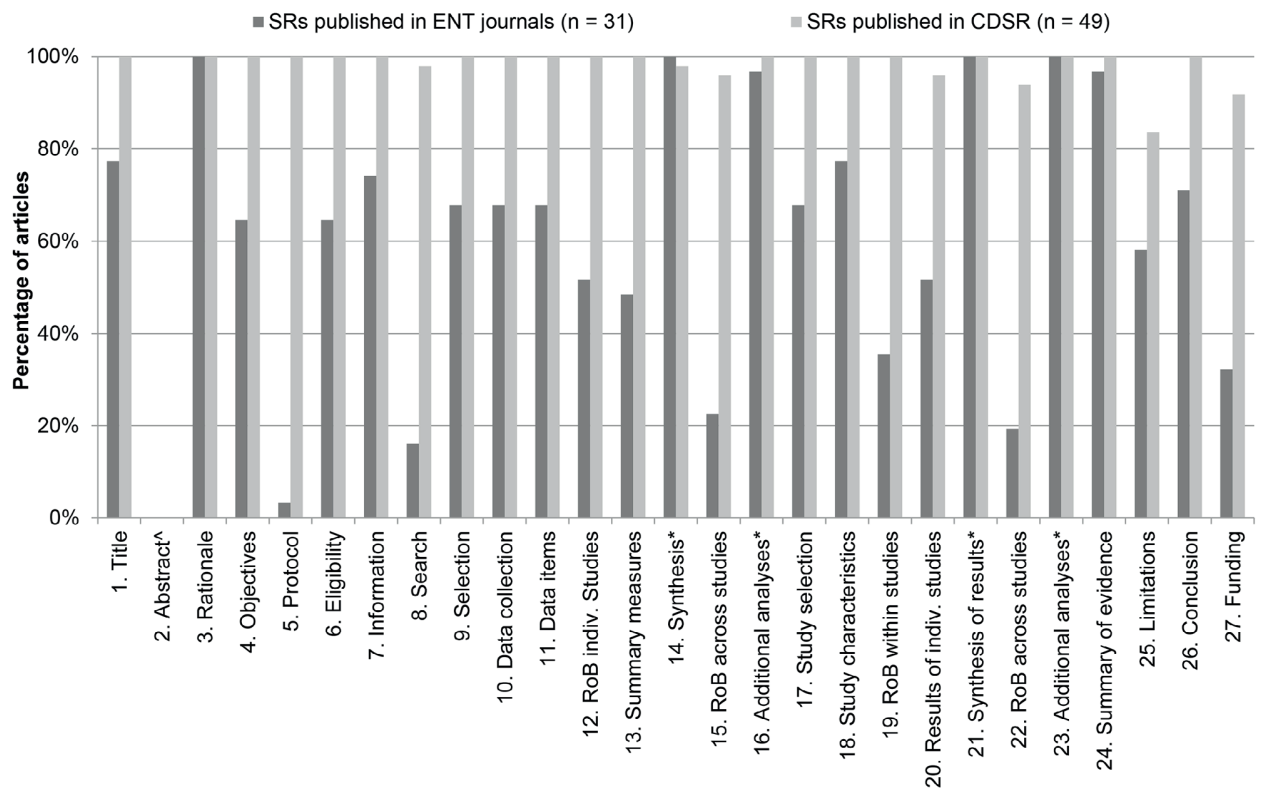

Legend: The percentage of articles that adequately reported PRISMA items is plotted per journal type (ENT journals [dark grey bars] -vs- CDSR [light grey bars]). For exact percentages, see Table 2.

$\wedge$ Item 2 is scored separately, see Figure 3. Items 14, 16, 21 and 23 are optional items. For details on scoring, see Supporting Information 2.

SRs and MAs published in ENT journals reported several individual items inadequately more frequently than SRs and MAs published in the CDSR. First, only 3\% of SRs published in ENT journals refer to a published review protocol of their studies (item 5). Second, less than 20\% of SRs or MAs reported their full search syntax (item 8), either in the main text or in online supplementary material. Third, more than three quarters of the studies did not report the assessment of risk of bias across studies (items 15 and 22) adequately, possibly reflecting that the risk of bias across studies was not considered in their reviews. Moreover, the articles failed to report an assessment of the risk of bias within the included studies adequately (item 19). Finally, more than two thirds of the articles did not report their source of funding, thereby omitting possible conflicts of interest (item 27). Unlike the articles published in ENT journals, 
SRs from the CDSR reported a vast majority of PRISMA item adequately.

In sum, the 31 articles published in the top 5 ENT journals reported a median of 54.4\% (mean 62.2\%, 95\% CI: 54.4\%-71.7\%) of the PRISMA items adequately, whereas the 49 articles published in the CDSR reported a median of $100.0 \%$ (mean 98.2\%, 97.3\%-99.1\%) adequately. The difference in the reporting of PRISMA items between the two journal types is statistically significant $(p<0.001)$.

\section{PRISMA for Abstracts}

The exact percentages of adequately reported PRISMA for Abstracts items for SRs and MAs published in ENT journals and CDSR are reported in Table 3 (together with statistical significance), with a graphic illustration in Figure 3.

Table 3. Data table of Figure 3, number of adequately reported PRISMA for Abstract items per journal type

\begin{tabular}{|c|c|c|c|c|c|c|c|c|c|c|c|c|}
\hline Item & 1 & 2 & 3 & 4 & 5 & 6 & 7 & 8 & 9 & 10 & 11 & 12 \\
\hline $\begin{array}{l}\text { SRs } \\
\text { published } \\
\text { in ENT } \\
\text { journals ( } \mathrm{n} \\
=31 \text { ) }\end{array}$ & $77,4 \%$ & $38,7 \%$ & $12,9 \%$ & $9,7 \%$ & $12,9 \%$ & $35,5 \%$ & $74,2 \%$ & $71,0 \%$ & $25,8 \%$ & $87,1 \%$ & $0,0 \%$ & $0,0 \%$ \\
\hline $\begin{array}{l}\text { SRs } \\
\text { published } \\
\text { in CDSR ( } \mathrm{n} \\
=49 \text { ) }\end{array}$ & $100,0 \%$ & $49,0 \%$ & $100,0 \%$ & $100,0 \%$ & $57,1 \%$ & $100,0 \%$ & $100,0 \%$ & $100,0 \%$ & $91,8 \%$ & $98,0 \%$ & $0,0 \%$ & $0,0 \%$ \\
\hline$p$-value ${ }^{\$}$ & $<0.001$ & 0.359 & $<0.001$ & $<0.001$ & $<0.001$ & $<0.001$ & $<0.001$ & $<0.001$ & $<0.001$ & 0.051 & 0.423 & 0.423 \\
\hline
\end{tabular}

\section{Legend:}

The percentage of articles that adequately reported PRISMA for Abstract items per journal type, ENT journals -vsCDSR.

$\$$ Chi-square test. $P$-values in italic typeface highlight a difference that was not statistically significantly different between the two journal types. 
Figure 3. Number of adequately reported PRISMA for Abstract items per journal type.

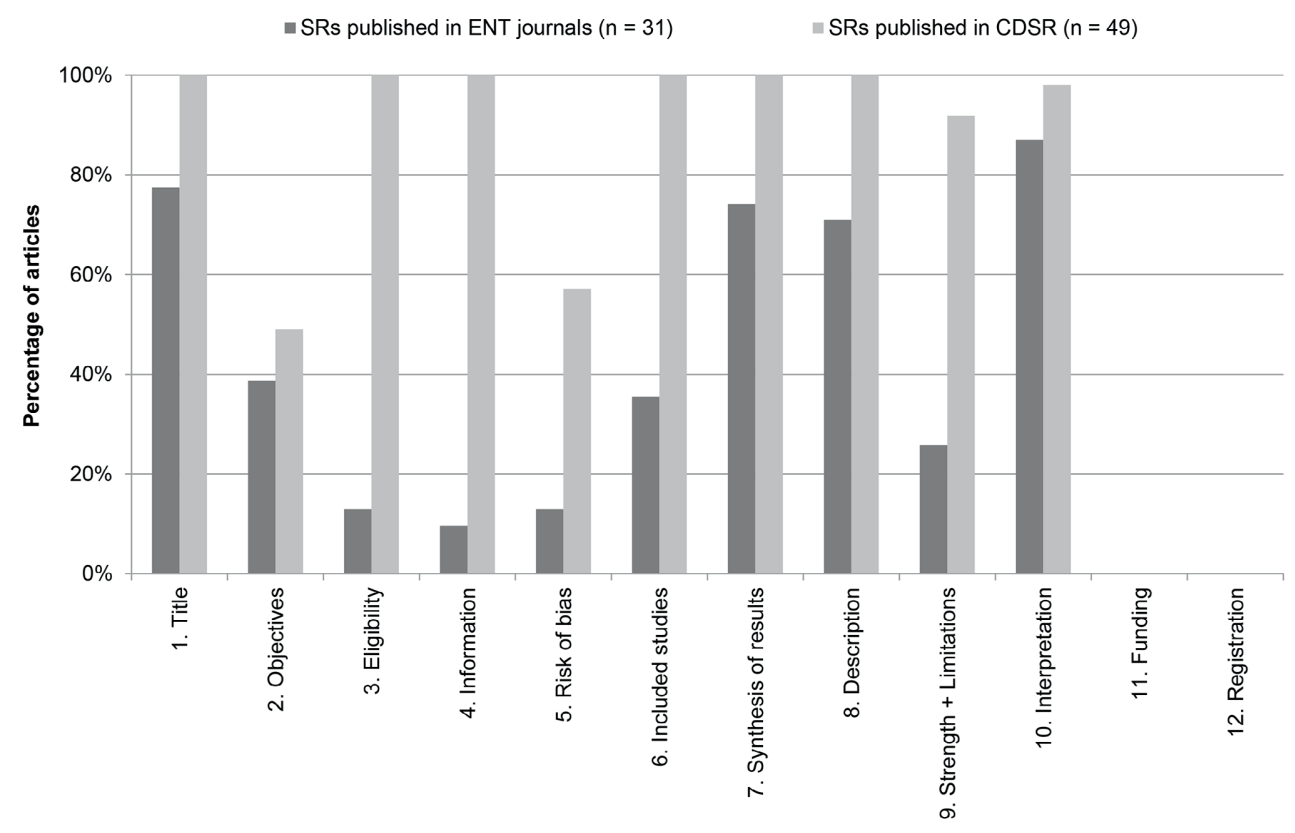

Legend: The percentage of articles that adequately reported PRISMA for Abstract items is plotted per journal type (ENT journals [dark grey bars] -vs- CDSR [light grey bars]). For exact percentages, see Table 3. For details on scoring, see Supporting Information 3.

Only $\sim 10 \%$ of articles published in ENT journals reported the methods of their studies adequately (items 3-5, Eligibility criteria, Information sources and Risk of bias, respectively). Moreover, the strengths and limitations (item 9) of their studies are adequately discussed in the abstracts of only one quarter of the studies.

In contrast to the full text assessment, SRs published in the CDSR reported individual items of the PRISMA for Abstracts checklist inadequately more frequently. For instance, often a specific outcome was not reported in the abstract, therefore failing to adequately report Objectives (item 2) according to the PICOS structure (Patient, Intervention, Comparison, Outcome and Study design). Also the assessment of the risk of bias was not always reported in the abstracts. Although it had always been performed, we assessed whether it was reported adequately in the abstract or not. Finally, SRs and MAs published in both ENT journals and 
in the CDSR failed to report details on funding and registration in their abstracts (items 11 and 12).

In sum, the articles published in ENT journals reported a median of $41.7 \%$ (mean $31.7 \%, 30.2 \%-44.0 \%)$ of PRISMA for Abstracts items adequately, whereas the articles published in the CDSR reported a median of 75.0\% (mean 75.2\%, 73.1\%-76.2\%) adequately. The difference in the reporting of PRISMA for Abstracts items between the two journal types is statistically significant $(p<0.001)$.

\section{DISCUSSION}

To our knowledge, this study is the first to assess the quality of reporting of SRs and MAs in otorhinolaryngologic literature. We found that the current quality of reporting is suboptimal and we identified areas in which improvement is needed. To help authors improve their reporting, the PRISMA Statement was developed. Articles by authors who adhered to the PRISMA Statement were associated with improved quality of reporting. ${ }^{6-10}$

We found several individual items of the PRISMA checklist that require extra attention (Figure 2) in the reporting of SRs and MAs published in ENT journals. First, an unpublished study protocol reduces reproducibility and prohibits comparison of the intended and final review procedures. Therefore, the review is prone to publication bias. ${ }^{14}$ Recently, the PRISMA-P (protocol) initiative was launched, helping authors with developing a review protocol. ${ }^{15,16}$ Second, if risk of bias within and across studies is not assessed and the quality of the included studies is not critically appraised, readers are unable to value the results of the review. Finally, it is important to be transparent about the financial support that the review received, as specific types of sponsorship may be associated with positive study results. ${ }^{17}$ The International Committee of Medical Journal Editors (ICMJE) has published a conflicts of interest form and checklist (http://www.icmje.org/conflicts-of-interest/), which may help in improving the transparency of financial support of review authors.

In our study, we identified a significant difference between the quality of reporting 
of SRs and MAs published in ENT journals versus those published in the CDSR: medians of $54.4 \%$ versus $100.0 \%$ of PRISMA items were reported adequately. In other research fields, similar deficits were identified previously. For example, in dentistry, orthodontics and radiology, the reporting of trial registration, funding and risk of bias within and across studies was suboptimal. ${ }^{7,9,18}$ In line with our findings, the quality of reporting of SRs and MAs published in the CDSR was significantly better than that of SRs and MAs published in subject specific journals. ${ }^{7,16,19}$

This study also assessed PRISMA for Abstracts items separately. The methodology and discussion section of abstracts are generally not reported adequately by SRs and MAs published in ENT journals (Figure 3). Abstracts of SRs and MAs published in ENT journals reported a median of $41.7 \%$ of PRISMA for Abstracts items adequately versus $75.0 \%$ for SRs and MAs published in CDSR. Likewise, an extensive recent study by Hopewell et al. found many inadequately reported items (participants, harms, strengths and limitations and funding source) in conference abstracts. ${ }^{20}$

Compliance with reporting guidelines (either QUORUM or PRISMA) is associated with improved reporting. ${ }^{5-10,19}$ Therefore, we think it is important that journals editors endorse reporting guidelines to help authors improve reporting. The CDSR already refers to the PRISMA Statement in their instructions to authors. However, none of the top 5 ENT journals endorse the PRISMA Statement (www.prisma-statement.com/endorsers.htm), nor do they refer to the Statement in the instructions to authors section on their websites.

A strength of our study is that all items were scored separately by two authors, and an independent otorhinolaryngologist was consulted if no consensus was reached. Moreover, the two authors independently selected the studies to be included. Furthermore, we included SRs and MAs from 2012 and 2013, resulting in a sufficient number of articles for our analyses. Lastly, we transparently provided all details of the search (Figure 1 and Supporting Information 1) and the scoring of items (Supporting Information 2 and 3).

Our study also has limitations. One may wonder if the comparison of SRs and MAs published in ENT journals to SRs and MAs published in the CDSR is valid. We do not 
advocate that all SRs and MAs in otorhinolaryngology should be Cochrane SRs because of their superior methodological quality, since the process of writing a Cochrane SR is timeconsuming and the length of Cochrane SRs may deter clinicians from using them. However, the quality of reporting of SRs and MAs published in the CDSR is superior to the quality of reporting of SRs and MAs published in ENT journals. The PRISMA checklist is a useful tool to help authors report SRs and MAs better. This analysis of the quality of reporting of SRs and MAs in ENT may serve as a benchmark for future assessments of the quality of reporting of SRs and MAs in otorhinolaryngology.

\section{$\underline{\text { Recommendation }}$}

Transparent reporting of what was done and found in SRs and MAs ensures that clinicians can value the results. To help authors improve the quality of reporting of SRs and MAs, the PRISMA Statement was developed. ${ }^{3,4}$ We advise authors to report their otorhinolaryngologic SRs and MAs according to the PRISMA Statement, as adherence to the PRISMA Statement is associated with improved quality of reporting. ${ }^{6-10}$ We suggest to implement the PRISMA-P Statement in the development of a protocol for a SR or MA. ${ }^{15,16}$ Furthermore, we recommend editors of ENT journals to endorse the PRISMA Statement in the Instructions to Authors section on their websites. A next step to raise awareness of the importance of adequate reporting is an active implementation strategy of reporting guidelines. This strategy has been shown to be more effective in improving the quality of reporting, but this requires editorial effort and time..$^{21,22}$

\section{CONCLUSION}

The quality of reporting of SRs and MAs in ENT journals is suboptimal compared to the quality of reporting of SRs and MAs in CDSR. Large differences in individual items exist. As reporting according to the PRISMA Statement is associated with improved quality of reporting, authors and editors of ENT journals should adhere to the PRISMA Statement. 


\section{SUPPORTING INFORMATION}

Supporting Information can be accessed online via

https://journals.plos.org/plosone/article?id=10.1371/journal.pone.0136540

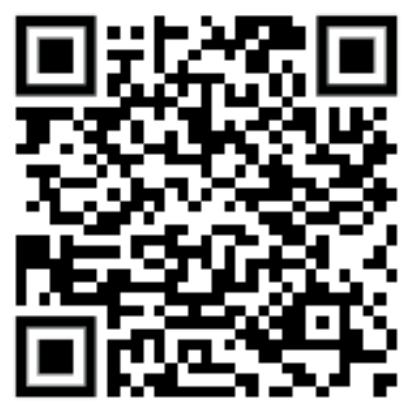

Supporting Information 1 Complete search syntax

Supporting Information 2 Explanation of criteria to score as 'adequately reported PRISMA item'

Supporting Information 3 Explanation of criteria to score as 'adequately reported PRISMA for Abstracts item' 


\section{REFERENCES}

1. Oxford Centre for Evidence-based Medicine - Levels of Evidence (March 2009) via http://www.cebm.net/oxford-centre-evidence-based-medicine-levels-evidencemarch-2009/, accessed 18 November 2014

2. Moher D, Cook DJ, Eastwood S, Olkin I, renne D, Stroup DE. Improving the quality of reports of meta-analyses of randomised controlled trials: the QUOROM statement. Quality of Reporting of Meta-analyses. Lancet 1999;354(9193):1896-1900.

3. Moher D, Liberati A, Tetzlaff J, Altman DG, for the PRISMA Group. Preferred reporting items for systematic reviews and meta-analyses: the PRISMA statement. PLoS Med 2009;6(7):e10000097.

4. Liberati A, Altman DG, Tetzlaff J, Mulrow C, Gøtzsche PC, Ioannidis JP, Clarke M, Devereaux PJ, Kleijnen J, Moher D. The PRISMA statement for reporting systematic reviews and meta-analyses of studies that evaluate healthcare interventions: explanation and elaboration. BMJ 2009;339:b2700.

5. Delaney A, Bagshaw SM, Ferland A, Manns B, Laupland KB, Doig CJ. A systematic evaluation of the quality of meta-analyses in the critical care literature. Crit Care 2005;9(5):R575-582.

6. Tao KM, Li XQ, Zhou QH, Moher D, Ling CQ, Yu WF. From QUOROM to PRISMA: a survey of high-impact medical journals' instructions to authors and a review of systematic reviews in anesthesia literature. PLoS One 2011;6(11):e27611.

7. Fleming PS, Seehra J, Polychronopoulou A, Fedorowicz Z, Pandis N. A PRISMA assessment of the reporting quality of systematic reviews in orthodontics. Angle Orthod 2013;83(1):158-163.

8. Panic N, Leoncini E, de Belvis G, Ricciardi W, Boccia S. Evaluation of the endorsement of the preferred reporting items for systematic reviews and meta-analysis (PRISMA) statement on the quality of published systematic review and meta-analyses. PLoS One 2013;8(12):e83138. 
9. Tunis AS, McInnes MD, Hanna R, Esmail K. Association of study quality with completeness of reporting: have completeness of reporting and quality of systematic reviews and meta-analyses in major radiology journals changed since publication of the PRISMA statement? Radiology 2013;269(2):413-426.

10. Stevens A, Shamseer L, Weinstein E, Yazdi F, Turner L, Thielman J, Altman DG, Hirst A, Hoey J, Palepu A, Schulz KF, Moher D. Relation of completeness of reporting of health research to journals' endorsement of reporting guidelines: systematic review. BMJ 2014;348:g3804.

11. The editorial team, Cochrane Ear Nose and Throat Disorders Group. About the Cochrane Collaboration (Cochrane Reviews Group (CRGs)), 2012 issue 7, art. no.: ENT. CENTRAL search strategy. Available: http://onlinelibrary.wiley.com/o/cochrane/ clabout/articles/ENT/sect0-meta.html, accessed 3 September 2014.

12. National Institutes of Health, US National Library of Medicine. Search Strategy Used to Create the Systematic Reviews Subset on PubMed, available via http://www.nlm.nih.gov/ bsd/pubmed subsets/sysreviews strategy.html, accessed 3 September 2014.

13. Beller EM, Glasziou PP, Altman DG, Hopewell S, Bastian H, Chalmers I, Gøtzsche PC, Lasserson T, Tovey D, for the PRISMA for Abstracts Group. PRISMA for Abstracts: reporting systematic reviews in journal and conference abstracts. PLoS Med 2013;10(4):e1001419.

14. Silagy CA, Middleton P, Hopewell S. Publishing protocols of systematic reviews: comparing what was done to what was planned. JAMA 2002;287(21):2831-2834.

15. Moher D, Shamseer L, Clarke M, Ghersi D, Liberati A, Petticrew M, Shekelle P, Stewart LA, for the PRISMA-P Group. Preferred reporting items for systematic review and metaanalysis protocols (PRISMA-P) 2015 statement. Syst Rev 2015;4(1):1.

16. Shamseer L, Moher D, Clarke M, Ghersi D, Liberati A, Petticrew M, Shekelle P, Stewart LA, for the PRISMA-P Group. Preferred reporting items for systematic review and metaanalysis protocols (PRISMA-P) 2015: elaboration and explanation. BMJ 2015;350:g7647. 
17. Sun GH, Houlton JJ, MacEachern MP, Bradford CR, Hayward RA. Influence of study sponsorship on head and neck cancer randomized trial results. Head Neck 2013;35(10):1515-1520.

18. Seehra J, Fleming PS, Polychronopoulou A, Pandis N. Reporting completeness of abstracts of systematic reviews published in leading dental specialty journals. Eur J Oral Sci 2013;121(2):57-62.

19. Moher D, Tetzlaff J, Tricco AC, Sampson M, Altman DG. Epidemiology and reporting characteristics of systematic reviews. PLoS Med 2007;4(3):e78.

20. Hopewell S, Boutron I, Altman DG, Ravaud P. Deficiencies in the publication and reporting of the results of systematic reviews presented at scientific medical conferences. J Clin Epidemiol 2015;68(12):1488-1495.

21. Pandis N, Shamseer L, Kokich VG, Fleming PS, Moher D. Active implementation strategy of CONSORT adherence by a dental specialty journal improved randomized clinical trial reporting. J Clin Epidemiol 2014;67(9):1044-1048.

22. Hopewell S, Ravaud P, Baron G, Boutron I. Effect of editors' implementation of CONSORT guidelines on the reporting of abstracts in high impact medical journals: interrupted time series analysis. BMJ 2012;344:e4178. 



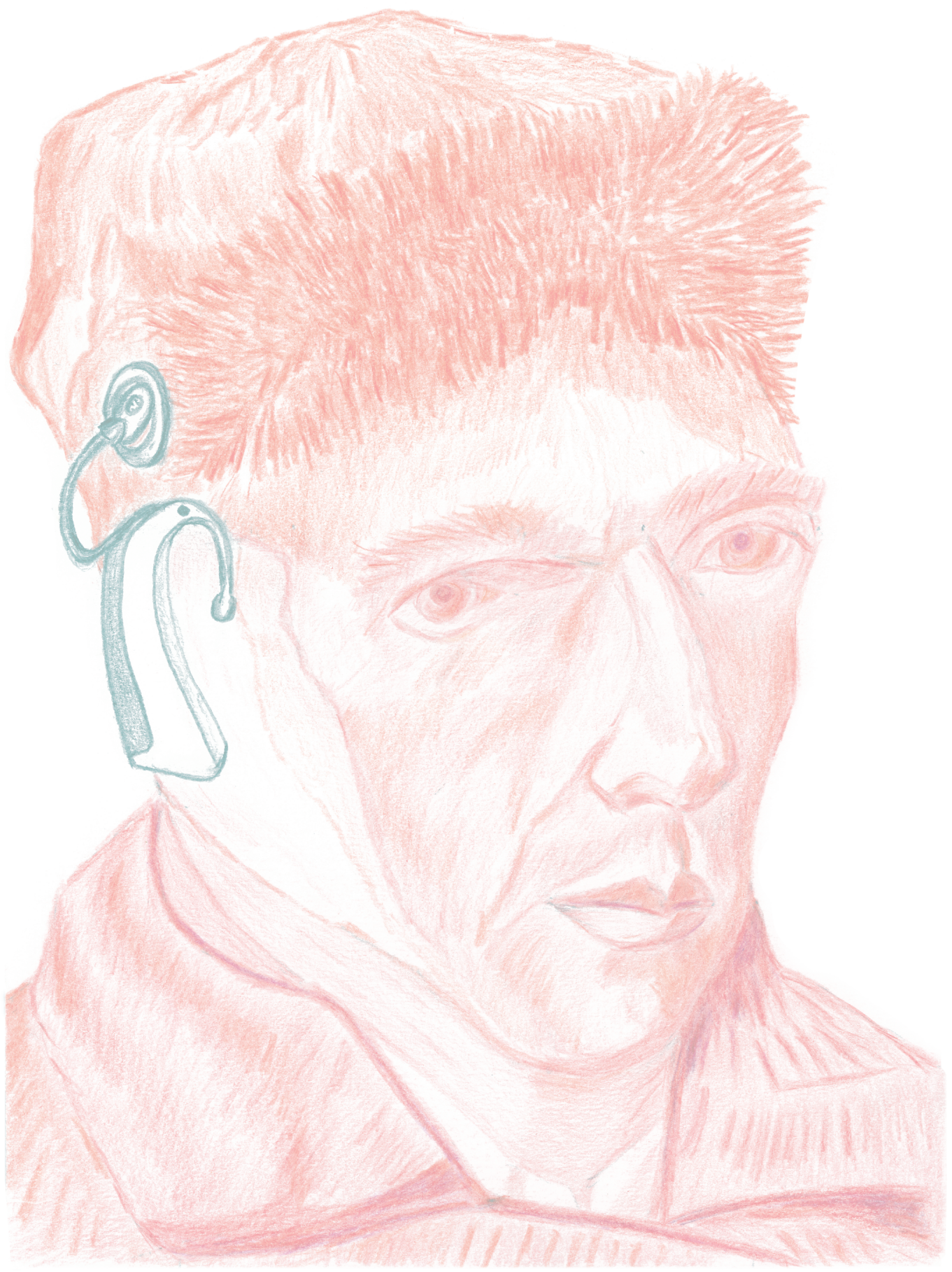




\section{0}

\section{Assessment of the Quality of Reporting of Randomised Controlled Trials in}

Otorhinolaryngologic Literature - Adherence to the CONSORT Statement 


\section{ABSTRACT}

\section{Background}

Randomised Controlled Trials (RCTs) are the preferred study design when comparing therapeutical interventions in medicine. To improve clarity, consistency and transparency of reporting RCTs, the Consolidated Standards of Reporting Trials (CONSORT) statement was developed.

\section{Objectives}

Our objectives were (1) to assess the quality of reports and abstracts of RCTs in otorhinolaryngologic literature by using CONSORT checklists, (2) to compare the quality of reports and abstracts of otorhinolaryngologic RCTs between the top 5 general medical journals and the top 5 otorhinolaryngologic journals, and (3) to formulate recommendations for authors and editors of otorhinolaryngologic (or Ear, Nose, Throat; 'ENT') journals.

\section{Methods}

Based on 2012 ISI Web of Knowledge impact factors, the top 5 general medical and top 5 ENT journals were selected. On 25 June 2014, using a highly sensitive Cochrane RCT filter and ENT filter, possibly relevant articles since January $1^{\text {st }}, 2010$ were retrieved and relevant RCTs were selected. We assessed how many CONSORT items were reported adequately in reports and abstracts and compared the two journal types.

\section{Results}

Otorhinolaryngologic RCTs $(n=15)$ published in general medical journals reported a mean of $92.1 \%$ (95\% confidence interval: $89.5 \%-94.7 \%$ ) of CONSORT items adequately, whereas RCTs $(\mathrm{n}=18)$ published in ENT journals reported a mean of $71.8 \%(66.7 \%-76.8 \%)$ adequately $(p<0.001)$. For abstracts, means of 70.0\% (63.7\%-76.3\%) and 32.3\% (26.6-38.0\%) were found, respectively $(p<0.001)$. Large differences for specific items exist between the two journal types. 


\section{Conclusion}

The quality of reporting of RCTs in otorhinolaryngologic journals is suboptimal. RCTs published in general medical journals have a higher quality of reporting than RCTs published in ENT journals. We recommend authors to report their trial according to the CONSORT Statement and advise editors to endorse the CONSORT Statement and implement the CONSORT Statement in the editorial process to ensure more adequate reporting of RCTs and their abstracts. 


\section{INTRODUCTION}

Randomised Controlled Trials (RCTs) are the preferred type of study design when comparing therapeutical interventions in medicine. This study design prevents selection and confounding bias and permits blinding of participants and researchers. ${ }^{1}$

However, poor reporting of RCTs can reduce their usefulness. ${ }^{2,3}$ Clinicians and researchers must have access to clear, transparent and complete information to assess the quality and results of a trial accurately. Various aspects of RCTs may lead to bias when reported inadequately. To be able to assess the risk of publication bias and selective reporting, trials are advised to be registered in trial registers before patient enrolment and trial protocols should be published. ${ }^{4,5}$ Finally, adequate reporting of the randomisation procedure, allocation concealment and blinding is essential to value the trial and its results. ${ }^{3,6,7}$

To improve clarity and transparency of reporting of trials, the Consolidated Standards of Reporting Trials (CONSORT) statement (www.consort-statement.org) was developed in $1996^{8}$ and revised in $2001^{9}$ and $2010^{10,11}$. The CONSORT Statement and the corresponding checklist summarize all essential items that should be reported in an RCT. Several extensions have been published since, e.g. CONSORT for Abstracts ${ }^{12}$, CONSORT for harms ${ }^{13}$ and CONSORT for non-pharmacological treatments ${ }^{14}$. Journal endorsement of the CONSORT Statement may beneficially influence the completeness of reporting of trials. ${ }^{15-17}$

The objective of this study was to assess the quality of reports and abstracts of RCTs in otorhinolaryngologic literature. Therefore, we scored articles in the otorhinolaryngologic research field with CONSORT checklists. Second, we compared the quality of reporting RCTs between the top 5 general medical journals and the top 5 otorhinolaryngologic journals. Finally, we aimed to formulate recommendations for authors and editors of otorhinolaryngologic journals. 


\section{METHODS}

\section{Journals}

We selected the top 5 general medical journals and top 5 otorhinolaryngologic journals (Ear Nose Throat, 'ENT journals'), based on their 2012 ISI Web of Knowledge impact factors (www.webofknowledge.com, date of access June $25^{\text {th }}, 2014$ ). The journals and impact factors are shown in Table 1. The top 5 journals in general medical literature are New England Journal of Medicine (NEJM), followed by The Lancet (Lancet), Journal of the American Medical Association (JAMA), British Medical Journal (BMJ) and PLOS Medicine (PLOS Med). In ENT literature, Ear \& Hearing (Ear Hear) is the journal with the highest impact factor, followed by Journal of the Association for Research in Otolaryngology (JARO), Head \& Neck (Head Neck), Hearing Research (Hear Res) and Audiology \& Neurotology (Audiol Neurotol).

Table 1. Impact Factors 2012 Top 5 general medical and ENT journals.

\begin{tabular}{lr}
\hline Journal & Impact Factor $^{*}$ \\
\hline General medical journals & 51.658 \\
\hline 1. New England Journal of Medicine (NEJM) & 39.060 \\
2. The Lancet (Lancet) & 29.978 \\
3. Journal of the American Medical Association (JAMA) & 17.215 \\
4. British Medical Journal (BMJ) & 15.253 \\
5. PLOS Medicine (PLOS Med) & 3.262 \\
\hline ENT journals & 2.952 \\
\hline 1. Ear \& Hearing (Ear Hear) & 2.833 \\
2. Journal of the Association for Research in Otolaryngoloy (JARO) & 2.537 \\
3. Head \& Neck (Head Neck) & 2.318 \\
4. Hearing Research (Hear Res) & \\
5. Audiology \& Neurotology (Audiol Neurotol) & \\
\hline
\end{tabular}

\section{Legend:}

* Source: ISI Web of Knowledge 2012, Journal Citations Reports (JCR) via www.webofknowledge.com, accessed on June $25^{\text {th }}, 2014$.

ENT $=$ Ear Nose Throat. 


\section{$\underline{\text { Search }}$}

We searched Pubmed on June $25^{\text {th }}, 2014$ for relevant literature using two filters developed and tested by the Cochrane Collaboration. First, an adapted version of the ENT search filter was used to identify otorhinolaryngologic articles (Supporting Information 1 ). ${ }^{18}$ Second, to retrieve only RCTs, the highly sensitive RCT filter was used. ${ }^{19}$ A date restriction was applied to yield only articles published since January $1^{\text {st }}, 2010$ (for complete search: see Supporting Information 1). Finally, a combination was made with a search syntax for the top 5 general medical journals and the top 5 ENT journals respectively using Boolean operator AND.

\section{Study selection}

Two authors (JPMP and IS) independently assessed titles and abstracts of the retrieved articles to check if the study was indeed conducted in the otorhinolaryngologic field and if it was an RCT. To be considered as a study in the otorhinolaryngologic field, studies must assess patients generally treated by otorhinolaryngologists or investigate a procedure generally performed by otorhinolaryngologists, including head and neck surgery (Supporting Information 1). To be considered an RCT, studies must have randomised their human population in two or more groups receiving a therapeutic intervention. Secondary analysis of previously reported RCTs or economic evaluations were excluded. Discrepancies between the two independent reviewers were discussed until consensus was reached.

\section{$\underline{\text { CONSORT } 2010 \text { adherence }}$}

To score the quality of reporting, the most recent version of the CONSORT Statement was used

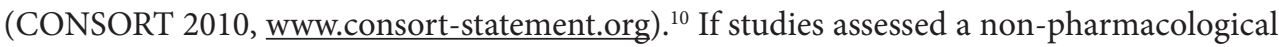
treatment, the additions stated in the CONSORT Statement for non-pharmacological interventions were taken into account and scored according to the descriptions in this extension. ${ }^{14}$ When scoring item 19 (Harms), we took the additions for reporting harms as explained in the CONSORT extension for reporting harms into account. ${ }^{13}$ 
The included articles were read full text independently by two authors (JPMP and IS) and differences in opinion were discussed until consensus was reached. We evaluated the number of items of the CONSORT 2010 checklist that were adequately reported. A more detailed explanation on how items were assessed can be found in Supporting Information 2. Next, we compared reporting of specific items between the general medical journals and ENT journals.

\section{CONSORT for Abstracts adherence}

We scored item 1b (Structured summary) of the CONSORT checklist separately on the checklist specifically designed to assess abstracts, published along with the CONSORT for Abstracts extension. ${ }^{12}$ A more detailed explanation on how all 16 items were assessed can be found in Supporting Information 3. Again, we also compared the adequate reporting of items per journal type.

Furthermore, we hypothesised that abstracts with more words would report more items adequately. To test this hypothesis, we counted the words of the abstract excluding title, authors names, affiliations, journal and volume numbers, and key words. These counts are shown with standard deviations and range.

\section{$\underline{\text { Data analysis }}$}

In total, there are 37 CONSORT (sub)items. Item 1b (Structured summary) was scored separately. The number of adequately reported items was thus divided by a possible total of 36, resulting in a percentage. The higher the percentage, the more adequately authors reported their trial. For the assessment of abstracts, the number of adequately reported items was divided by a possible total of 16 , because one of the items on the checklist is specific to conference abstracts only (name of authors), and therefore not scored.

Means, medians and 95\% confidence intervals (CI) were calculated. The two-tailed Mann-Whitney $\mathrm{U}$ test for 2 independent samples was used to compare CONSORT scores 
and CONSORT for Abstracts scores for articles published in general medical journals with ENT journals. The correlation between adequate reporting of CONSORT for Abstracts items and number of words was calculated using Spearman's rho. Statistical tests were performed using SPSS v20 statistics package. A $p$-value of $<0.05$ was considered statistically significant.

\section{RESULTS}

\section{Search}

The search process is shown in Figure 1. The combined search syntaxes yielded 40 articles in the general medical journals and 69 articles in the ENT journals.

Figure 1. Flowchart of search

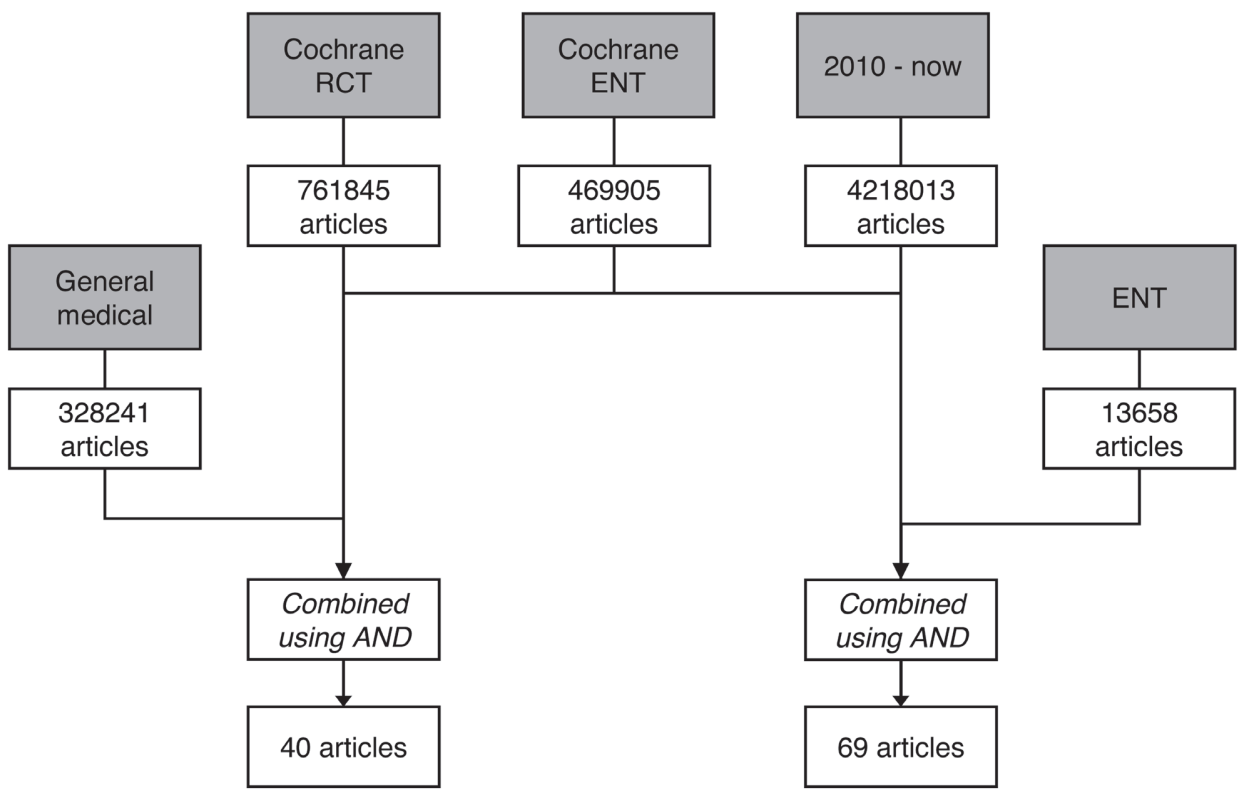

Legend: Date of search: June $25^{\text {th }}, 2014$. For complete search syntaxes: see Supporting Information 1. 


\section{$\underline{\text { Study selection }}$}

Of the 40 articles in the general medical journals, 3 articles were neither considered otorhinolaryngologic research nor an RCT and were therefore excluded. Seventeen RCTs were not considered otorhinolaryngologic and finally 17 otorhinolaryngologic studies were no RCTs. We included the remaining 15 RCTs in otorhinolaryngology (Table 2).

Of the 69 articles in the ENT journals, 1 article was excluded because it was neither an RCT nor conducted in otorhinolaryngology. We excluded 5 RCTs not performed in otorhinolaryngology and excluded 45 non-RCT studies in otorhinolaryngology. This large number of exclusions can be explained by phrases like 'stimuli were presented randomly' or 'in random order' that were detected by our highly sensitive RCT filter. In the end, we included 18 RCTs on otorhinolaryngologic topics (Table 3).

Tables 2 and 3. Assessment of retrieved articles in general medical journals (left) and ENT journals (right)

\begin{tabular}{|c|c|c|c|c|}
\hline & \multicolumn{2}{|c|}{ ENT? } & \\
\hline & & yes & no & \\
\hline \multirow[t]{2}{*}{ RCT? } & yes & 15 & 17 & 32 \\
\hline & no & 5 & 3 & 8 \\
\hline & & 20 & 20 & 40 \\
\hline
\end{tabular}

\begin{tabular}{|l|l|l|l|l|}
\cline { 3 - 4 } \multicolumn{2}{c|}{} & \multicolumn{2}{l|}{ ENT? } & \multicolumn{1}{|l|}{} \\
\cline { 2 - 4 } \multicolumn{2}{c|}{ RCT? } & yes & no & \\
\cline { 2 - 4 } & yes & 18 & 5 & $\mathbf{2 3}$ \\
\hline & no & 45 & 1 & $\mathbf{4 6}$ \\
\hline & $\mathbf{6 3}$ & $\mathbf{6}$ & $\mathbf{6 9}$ \\
\cline { 2 - 3 } & & & \multicolumn{2}{c}{}
\end{tabular}

Legend:

ENT $=$ Ear Nose Throat, RCT $=$ Randomised Controlled Trial

\section{CONSORT 2010 adherence}

The 15 articles published in general medical journals (NEJM 4, Lancet 1, JAMA 7, BMJ 3, PLOS Med 0) reported a mean of $92.1 \%$ (95\% CI: 89.5\%-94.7\%; median 91.9\%) of CONSORT items adequately. The 18 articles published in ENT journals (Ear Hear 2, JARO 1, Head Neck 7, Hear Res 2, Audiol Neurotol 6) reported a mean of 71.8\% (66.7\%-76.8\%; median 74.3\%) of CONSORT items adequately. Importantly, between these two journal types, there is a statistically significant difference in adequately reported CONSORT 2010 items (MannWhitney U test, $p<0.001)$. 
We also compared the difference in reporting individual items between the general medical and ENT journals. Figure 2 shows how many articles reported individual CONSORT items adequately, sorted per journal type. Several striking differences can be observed. First, the authors of two out of three RCTs in ENT journals did not mention that they conducted an RCT in the title of their manuscript. Even though the authors of one in five RCTs in general medical journals also not stated this, the number is much higher for articles published in ENT journals. Second, articles in ENT journals hardly stated sample size calculations (28\%, item $7 \mathrm{a})$ and subsequently inadequately reported why the trial was ended (39\%, item 14b). Third, both articles in general medical journals and in ENT journals reported Randomisation items (items 8a and 8b, 9, 10; Sequence generation, Allocation concealment mechanism, Implementation, respectively) inadequately; however, articles in ENT journals reported worse $(44 \%, 33 \%, 11 \%, 22 \%$ respectively). Fourth, items 20 and 21 (Limitations and Generalisability, respectively) were adequately reported in only $\sim 40 \%$ of articles in ENT journals. Fifth, only $11 \%$ of articles published in ENT journals stated the name of trial registry and the registration number (item 23), whereas this was $100 \%$ in general medical journals. Lastly, not one article in ENT journals stated where the full trial protocol could be accessed (40\% for general medical journals, item 24). 
Figure 2. Reporting of CONSORT items per journal type.

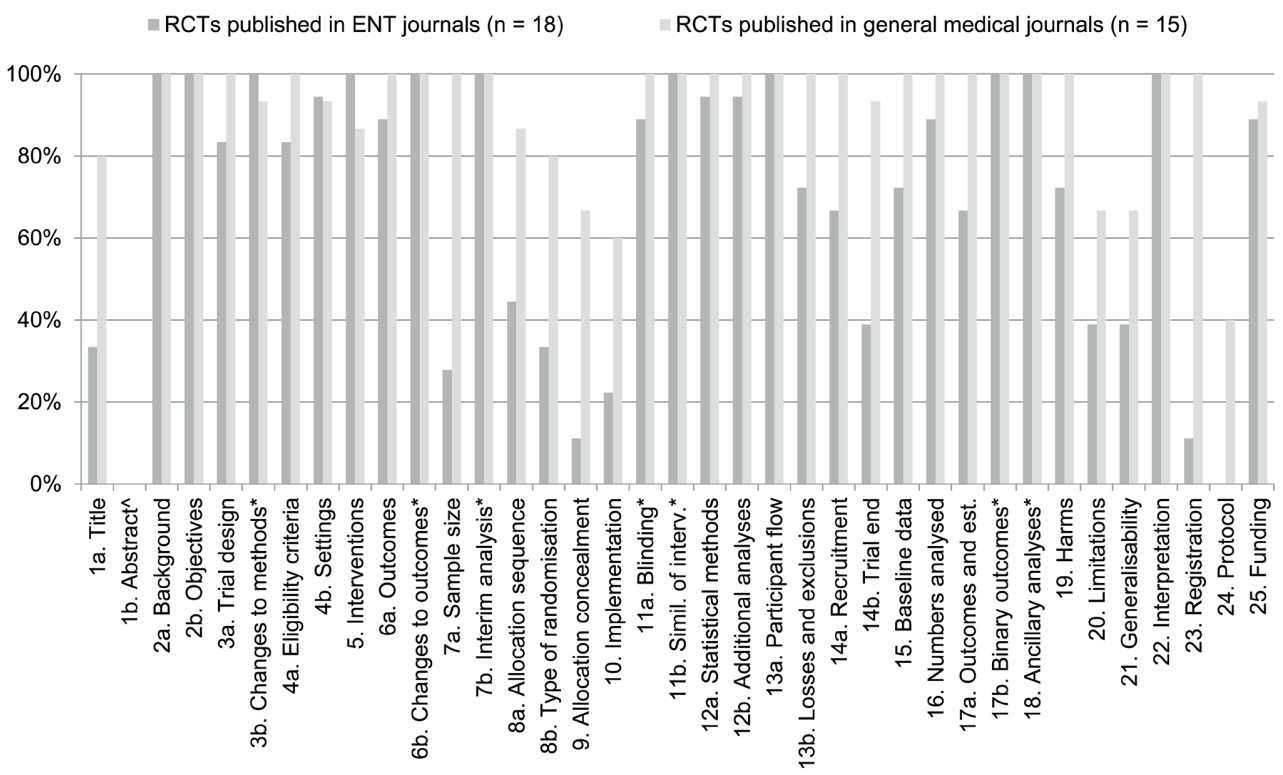

Legend: The percentage of articles reporting CONSORT items adequately (in \%), sorted per journal type (articles published in general medical journals, $\mathrm{n}=15$; articles published in ENT journals, $\mathrm{n}=18$ ), using the CONSORT 2010 checklist.

$\wedge$ Item 1b: item Structured summary is assessed with the specific CONSORT for Abstracts checklist, see Figure 3.

${ }^{*}$ Marked items concern optional items. When possible in the study and adequately reported, the item was scored as 'adequately reported. When possible, but not reported, the item was scored as 'inadequately reported'. If not possible, the item was not scored as 'inadequately reported', but left open.

\section{CONSORT for Abstracts adherence}

We assessed the reporting of abstracts with the CONSORT for Abstracts checklist separately. Articles published in general medical journals reported a mean of $70.0 \%$ (63.7\%-76.3\%; median 68.6\%) of CONSORT for Abstracts items, whereas articles published in ENT journals reported a mean of 32.3\% (26.6-38.0\%; median 31.6\%) of CONSORT for Abstracts items ( $p$ $<0.001)$.

When taking a closer look on individual CONSORT for Abstracts items, again there are large differences (Figure 3). First, no abstract of an article published in ENT journals adequately described Participants (item 3). Second, both abstracts of articles from ENT journals and general medical journals reported Randomisation (item 7$)$ inadequately (0 and 
19\%, respectively). Finally, Recruitment, Numbers analysed, Harms, Trial registration and Funding were adequately reported in $<20 \%$ of all articles published in ENT journals.

The abstracts of articles published in general medical journals had a mean of 337.7 \pm 35.7 [267-405] words, whereas abstracts of articles published in ENT journals had a mean of $204.3 \pm 69.1$ [84-331] words. This difference was statistically significant $(p<0.001)$. Furthermore, there was a significant correlation between abstract word count and number of adequately reported CONSORT for Abstracts items (Spearman's rho: $p=0.01$, correlation coefficient 0.736, sig (two-tailed) 0.000).

Figure 3. Reporting of CONSORT for Abstracts items per journal type.

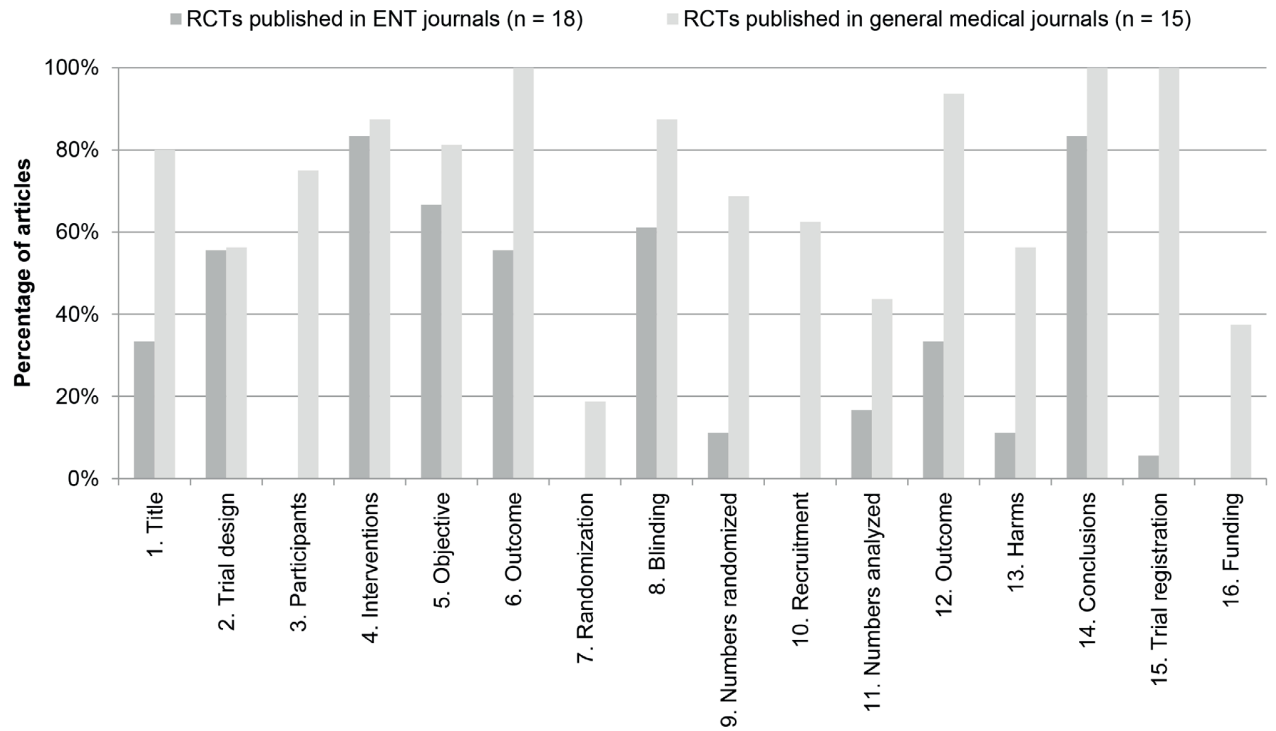

Legend: The percentage of articles reporting CONSORT for Abstract items adequately (in \%), sorted per journal type (articles published in general medical journals, $\mathrm{n}=15$; articles published in ENT journals, $\mathrm{n}=18$ ). On the original CONSORT for Abstracts checklist, item 2 (author names) is specific for conference abstracts only. Therefore, we renumbered the subsequent items. 


\section{DISCUSSION}

To our knowledge, this is the first study evaluating the quality of reports and abstracts of RCTs in otorhinolaryngologic literature. We evaluated the quality of reporting using the CONSORT (for Abstracts) checklists. ${ }^{10,12}$ We compared articles published in general medical journals with articles published in ENT journals and found adequate reporting of CONSORT items in $92.1 \%(89.5-94.7 \%)$ and $71.8 \%(66.7-76.8 \%)$ respectively $(p<0.001)$. Large differences in the quality of reporting RCTs were observed between both journal types.

There were several items essential in RCTs that were reported inadequately (Figure 2). First, $72 \%$ of articles published in ENT journals failed to report details of the sample size calculation (versus $0 \%$ failure in general medical journals). Also in other areas of expertise, less than half of the articles described sample size calculations correctly. ${ }^{1,17,20,21}$ The randomisation procedure is considered of utmost importance in RCTs. Yet articles did not report this process sufficiently: CONSORT items Sequence generation, Allocation concealment mechanism, Implementation were inadequately reported in $>50 \%$ of articles published in ENT journals. This figure was again congruent with other medical specialties ${ }^{1,3,21-23}$ and a recent Cochrane review $^{17}$ on the completeness of reporting RCTs. Studies published in ENT journals were not registered or did not mention trial registration (89\%) in a trial register and none of the articles had a previously published study protocol, yielding a risk of bias by selective reporting. ${ }^{4,6}$ Clinical trial registration before patient enrolment is part of the Declaration of Helsinki and is required by the International Committee of Medical Journal Editors (ICMJE). ${ }^{24}$ The articles published in general medical journals scored better on all these items. It has been highlighted before that reporting of essential items was better in general medical journals; it is thought to result from more stringent requirements by the editorial offices of the general medical journals. $^{7}$

In the analysis of reporting CONSORT for Abstracts items, again a large difference between articles published in general medical journals and ENT journals was observed (Figure 3). A significant correlation between the number of words and correctly reporting 
CONSORT for Abstract items was found (Spearman's rho, $p=0.01$ ), suggesting that authors simply need a sufficient amount of words to correctly report all necessary study information in their abstracts. In Table 4, the maximum number of abstract words is sorted for the top 5 general medical and ENT journals, showing large differences between these types.

Table 4. Maximum number of words in abstracts and endorsement of CONSORT Statement, per journal.

\begin{tabular}{lll}
\hline Journal & Maximum number of words & Endorse CONSORT? \\
\hline General medical journals & & \\
\hline 1. NEJM & 250 & Yes \\
2. Lancet & 300 & Yes \\
3. JAMA & 350 & Yes \\
4. BMJ & No fixed limit, 'to encourage full reporting' & Yes \\
5. PLOS Med & 300 & Yes \\
\hline ENT journals & & \\
\hline 1. Ear Hear & 500 & No \\
2. JARO & 250 & No \\
3. Head Neck & 150 & No \\
4. Hear Res & NA & No \\
5. Audiol Neurotol & '10 lines' & No \\
\hline
\end{tabular}

Legend:

Source: Instructions to Authors section on journals' websites, accessed July $17^{\text {th }}, 2014$. NA = not available.

Table 4 also shows that all general medical journals have endorsed the CONSORT Statement, meaning that these journals refer to the CONSORT Statement in the Instructions to Authors section on their websites (NEJM, Lancet) or even request a completed checklist to be uploaded with the submission of their manuscript (BMJ, JAMA, PLOS Med; personal communication of first author with editors, July 2014). None of the ENT journals report the CONSORT Statement in the Instructions to Authors section on their website (date of access July $\left.8^{\text {th }}, 2014\right)$, even though there are more than 200 medical journals that endorse CONSORT worldwide. ${ }^{25,26}$

Strengths of our study include our transparent search strategy to retrieve all RCTs. It can be easily reproduced, since all complete syntaxes are provided or referred to 
(Supporting Information 1). Next, we were very thorough in our analysis and for instance contacted editorial offices for further information. We gave an overview of all CONSORT items and did not select a few CONSORT items that we considered most important. If possible, we used an appropriate CONSORT extension to assess individual articles or items, e.g. non-pharmacologic treatments, CONSORT for Abstracts or Harms. ${ }^{12-14}$ Furthermore, we compared our data with other (surgical) medical specialties to put our findings in a broader perspective. Finally, we transformed our results into practical recommendations for authors and editors (see Recommendations).

Our study also has some limitations. First, interpreting phrases in articles and assessing them on a qualitative scale will always remain subjective. We think we countered this sufficiently by ensuring that two independent reviewers assessed whether articles were RCTs and were conducted in the otorhinolaryngologic field and by making our scoring system publicly available (Supporting Information 2 and 3). Another limitation could be that we performed our search only in the Pubmed database, excluding other medical databases. However, Pubmed is one of the largest and most widely available databases comprising over 24 million citations. ${ }^{27}$ Furthermore, we used the CONSORT 2010 checklist to assess the quality of included articles. One may argue that authors of articles published in 2010 had no chance to report according to this version of the CONSORT Statement. Nonetheless, previous versions of the CONSORT Statement were long available to help authors report adequately. ${ }^{8,9}$ Ultimately, reporting according to the CONSORT Statement does not necessarily result in better reporting of studies. For example, the three studies that reported according to the CONSORT Statement that were published in general medical journals reported a mean of 98.2\% (96.4-100\%) of CONSORT items adequately. This is, however, a higher mean score than the mean score of $90.5 \%(88.0 \%-93.1 \%)$ of the articles not reporting according to the CONSORT Statement published in general medical journals 


\section{Recommendations}

Correct reporting of clinical trials is an important aspect of good research and essential for clinicians and researchers to value the results of trials. To assist adequate reporting, the CONSORT Statement is a helpful tool. We advise authors in the otorhinolaryngologic field to use the CONSORT Statement when reporting an RCT. It has been shown that reporting according to the CONSORT Statement has a beneficial influence on the quality of reporting. ${ }^{15-17}$ We recommend editors of ENT journals to endorse the CONSORT Statement in the Instructions for Authors section on their website and to make it a requirement that a CONSORT checklist is submitted along with a new manuscript. Finally, a larger maximum number of words should be allowed for abstracts of RCTs to assure accurate description of all essential trial information.

\section{CONCLUSION}

The quality of reports of RCTs in otorhinolaryngologic literature assessed with the CONSORT checklists is suboptimal. Therefore the value of these trials for clinicians and researchers is limited. RCTs published in general medical journals (that all endorse the CONSORT Statement) have a higher quality of reporting than articles published in ENT journals. We advise authors to report their trial according to the CONSORT Statement. Finally, we recommend editors to endorse the CONSORT Statement and to require the submission of a completed CONSORT checklist along with a new manuscript prior to peer-review to ensure more adequate reporting of RCTs. 


\section{SUPPORTING INFORMATION}

Supporting Information can be accessed online via:

https://journals.plos.org/plosone/article?id=10.1371/journal.pone.0122328

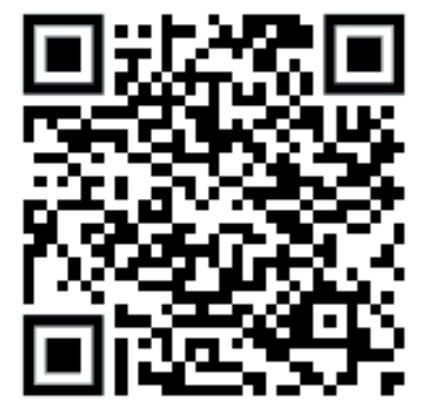

Supporting Information 1 Complete search syntax

Supporting Information 2 Explanation of criteria to score as 'adequately reported CONSORT item'

Supporting Information 3 Explanation of criteria to score as 'adequately reported CONSORT for Abstract item' 


\section{REFERENCES}

1. Agha R, Cooper D, Muir G. The reporting quality of randomised controlled trials in surgery: a systematic review. Int J Surg 2007;5(6):413-422.

2. Jüni P, Altman DG, Egger M. Systematic reviews in health care: assessing the quality of controlled clinical trials. BMJ 2001;323:42-46.

3. Chan AW, Altman DG. Epidemiology and reporting of randomised trials published in PubMed journals. Lancet 2005;365(9465):1159-1162.

4. Van de Wetering FT, Scholten RJ, Haring T, Clarke M, Hooft L. Trial registration numbers are underreported in biomedical publications. PLOS One 2012;7(11):e49599.

5. Chan AW, Hróbjartsson A, Haahr MT, Gøtzsche PC, Altman DG. Empirical evidence for selective reporting of outcomes in randomized trials. JAMA 2004;291(20):2457-2465.

6. Hewitt C, Hahn S, Torgerson DJ, Watson, J, Bland JM. Adequacy and reporting of allocation concealment: review of recent trials published in four general medical journals. BMJ 2005;30(7499):1057-1058.

7. Mills EJ, Wu P, Gagnier J, Devereaux PJ. The quality of randomized trial reporting in leading medical journals since the revised CONSORT statement. Contemp Clin Trials 2005;26(4):480-487.

8. Begg C, Cho M, Eastwood S, Horton R, Moher D, Olkin I, Pitkin R, Rennie D, Schulz KF, Simel D, Stroup DF. Improving the quality of reporting of randomized controlled trials. The CONSORT Statement. JAMA 1996;276:637-639.

9. Moher D, Schulz KF, Altman DG. The CONSORT Statement: revised recommendations for improving the quality of reports of parallel-group randomized trials. Lancet 2001;357(9263):1191-1194.

10. Schulz KF, Altman DG, Moher D, for the CONSORT group. CONSORT 2010 statement: updated guidelines for reporting parallel group randomised trials. BMJ 2010;340:c332.

11. Moher D, Hopewell S, Schulz KF, Montori V, Gøtzsche PC, Devereaux PJ, Elbourne D, Egger M, Altman DG, for the CONSORT group. CONSORT 2010 explanation and 
elaboration: updated guidelines for reporting parallel groups randomised trials. BMJ 2010;340:c869.

12. Hopewell S, Clarke M, Moher D, Wager E, Middleton P, Altman DG, Schulz KF, for the CONSORT group. CONSORT for reporting randomized controlled trials in journal and conference abstracts: explanation and elaboration. PLOS Med 2008;5(1):e20.

13. Ioannidis JP, Evans SJ, Gøtzsche PC, O’Neill RT, Altman DG, Schulz K, Moher D, for the CONSORT group. Better reporting of harms in randomized trials: an extension of the CONSORT Statement. Ann Intern Med 2004;141(10):781-788.

14. Boutron I, Moher D, Altman DG, Schulz K, Ravaud P, for the CONSORT group. Extending the CONSORT Statement to randomized trials of nonpharmacologic treatment: an explanation and elaboration. Ann Intern Med 2008;148:295-309.

15. Moher D, Jones A, Lepage L, for the CONSORT group. Use of the CONSORT statement and quality of reports of randomized trials: a comparative before-and-after evaluation. JAMA 2001;285(15): 1992-1995.

16. Plint AC, Moher D, Morrison A, Schulz K, Altman DG, Hill C, Gaboury I. Does the CONSORT checklist improve the quality of reports of randomised controlled trials? A systematic review. Med J Aust 2006;185(5):263-267.

17. Turner L, Shamseer L, Altman DG, Weeks L, Peters J, Kober T, Dias S, Schulz KF, Plint AC, Moher D. Consolidated standards of reporting trials (CONSORT) and the completeness of reporting randomized controlled trials (RCTs) published in medical journals. Cochrane Database Syst Rev 2012;11:MR000030

18. The editorial team, Cochrane Ear Nose and Throat Disorders Group. About the Cochrane Collaboration (Cochrane Reviews Group (CRGs)), 2012 issue 7, art. no.: ENT. CENTRAL search strategy. Available: http://onlinelibrary.wiley.com/o/cochrane/ clabout/articles/ENT/sect0-meta.html. Accessed 25 June 2014.

19. The Cochrane Collaboration (March 2011). Cochrane Highly Sensitive Search Strategy for identifying randomized trials in MEDLINE: sensitivity-maximizing version (2008 
revision); PubMed format. Cochrane Handbook for Systematic Reviews for Interventions, Box 6.4.a. Available handbook.cochrane.org. Accessed 25 June 2014.

20. Lai TY, Wong VW, Lam RF, Cheng AC, Lam DS, Leung GM. Quality of reporting of key methodological items of randomized controlled trials in clinical ophthalmic journals. Ophthalmic Epidemiol 2007;14(6):390-398.

21. Rios LP, Odueyungbo A, Moitri MO, Rahman, Thabane L. Quality of reporting randomized controlled trials in general endocrinology literature. J Clin Endocrinol Metab 2008;93(10):3810-3816.

22. Agha RA, Camm CF, Edison E, Orgill DP. The methodological quality of randomized controlled trials in plastic surgery needs improvement: a systematic review. J Plast Reconstr Aesthet Surg 2013;66(4):447-452.

23. Balasubramanian SP, Wiener M, Alshameeri Z, Tiruvoipati, Elbourne D, Reed MW. Standards of reporting randomized controlled trials in general surgery: can we do better? Ann Surg 2006;244(5):663-667.

24. International Committee of Medical Journal Editors (September 2004). Clinical Trial Registration, News \& Editorials. Available: http://www.icmje.org/news-and-editorials/ clin trial sep2004.pdf. Accessed 29 July 2014.

25. Altman DG. Endorsement of the CONSORT Statement by high impact medical journals: survey of instructions for authors. BMJ 2005;330:1056-1057.

26. Hopewell S, Altman DG, Moher D, Schulz KF. Endorsement of the CONSORT Statement by high impact factor medical journals: a survey of journal editors and journal 'Instructions to Authors'. Trials 2008;9:20.

27. National Institutes of Health, National Library of Medicine. Pubmed Help. http://www. ncbi.nlm.nih.gov/books/NBK3827/. Accessed 29 July 2014. 



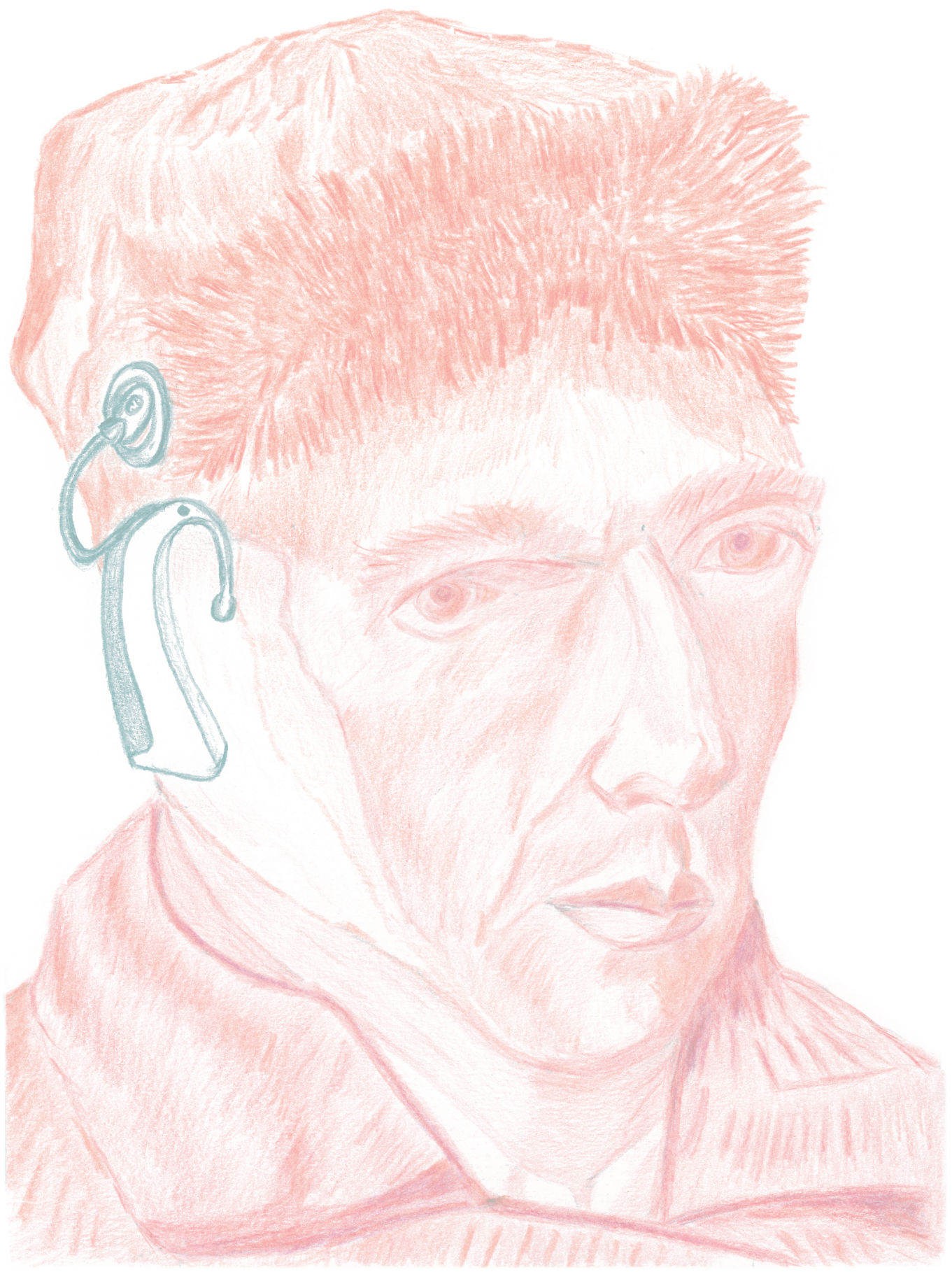




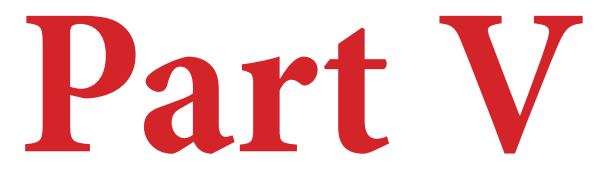

Discussion 


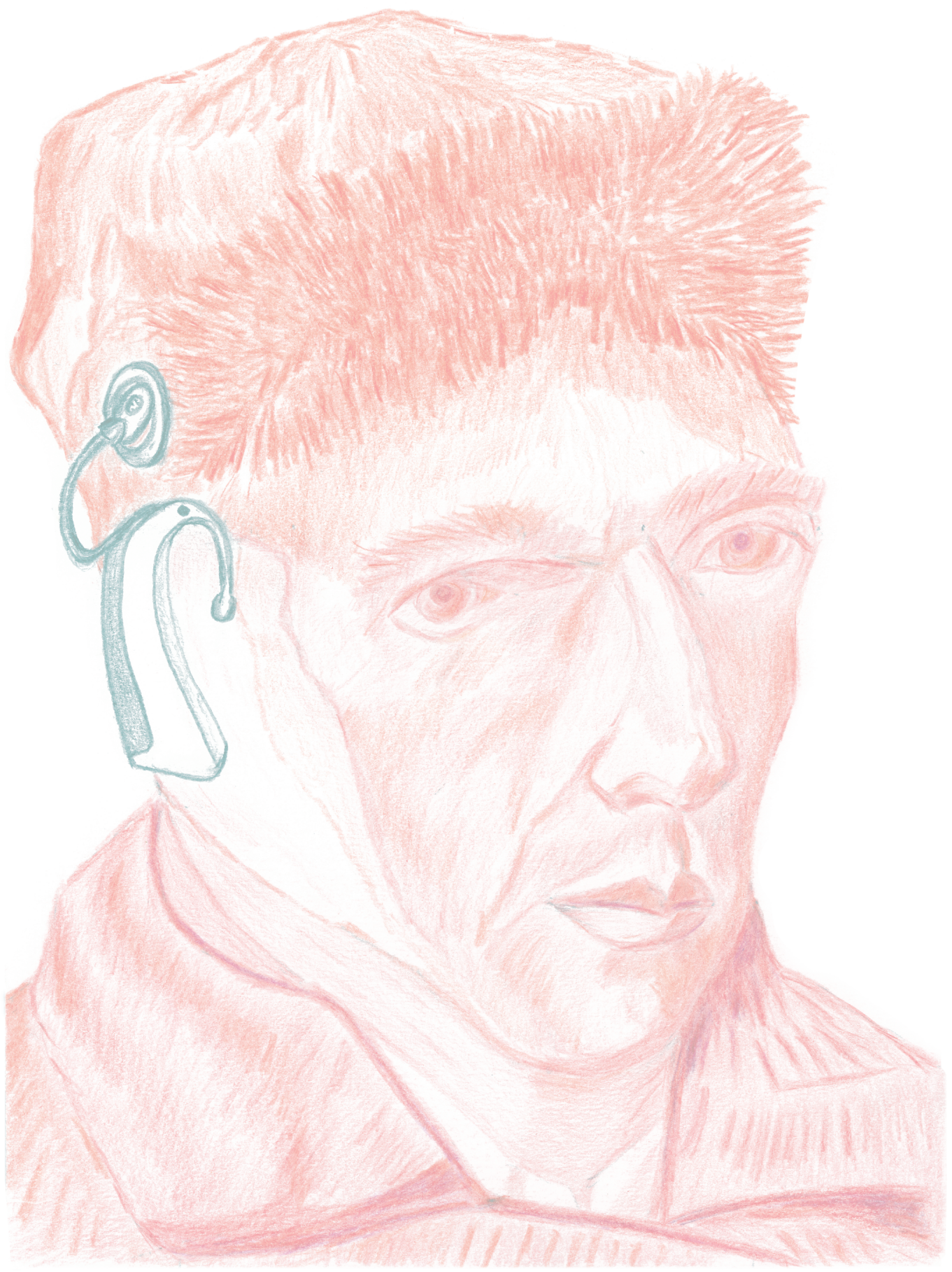




\section{1}

Summary and General Discussion 


\subsection{Summary and discussion}

\subsubsection{Part I: Introduction and literature reviews}

In this thesis, we published two literature reviews on the treatment options for single-sided deafness (SSD).

In Chapter 2, we systematically reviewed the literature on the clinical outcome of Bone Conduction Devices (BCDs) and Contralateral Routing of Sound hearing aids (CROS) for patients with SSD. Due to large heterogeneity between studies, pooling of results was not feasible. In this review no high level of evidence studies comparing BCD and CROS in patients with SSD were retrieved. The included studies were prone to selection bias, small sample sizes, double reporting of patients, and unclear inclusion criteria. All studies did not show a clear advantage of BCD or CROSS on speech perception in noise or sound localization compared to the unaided situation. Quality of life did not differ significantly after treatment with either BCD or CROS; however, subjective speech communication did improve with both treatment modalities.

Since publication of this review, no other comparative studies between BCD and CROS were published. One other systematic review comparing CROS hearing aids and BCDs for patients with SSD was published and its conclusions were concordant with ours. ${ }^{1}$

In Chapter 3, we performed a systematic review of the literature to evaluate the clinical outcomes of cochlear implantation for patients with SSD. Due to large heterogeneity between studies, we were not able to pool the results in a meta-analysis. Again, there were no high level of evidence studies concerning cochlear implantation in patients with SSD. The included studies had retrospective study designs, unclear eligibility criteria, small sample sizes with lack of statistical power, or no power calculations. All studies suggested important benefits of cochlear implantation regarding sound localization, quality of life (QoL) and tinnitus. Varying results were reported for speech perception in noise, possibly caused by the large clinical heterogeneity between studies (different test set-ups, configurations, languages, 
sentence tests etc.).

Several individual studies have been published investigating cochlear implantation for adults with SSD since publication of our systematic review, all with similar results. ${ }^{2-6}$ Though, all these studies were case series or cohort studies and were therefore prone to several forms of bias, as were the studies included in our systematic review. Moreover, none of these case series or cohort studies compared outcomes of CI to BCDs or CROS hearing aids.

With the identified lack of high-quality literature, we aimed to design a high-quality study to evaluate BCD, CROS and CI for patients with SSD. For therapeutic interventions, the study design with the highest level of evidence is a Randomized Controlled Trial (RCT).

\subsubsection{Part II: CINGLE-trial}

Based on the available scientific literature, we designed an RCT to evaluate the treatment options for SSD. In Chapter 4, the study protocol of this RCT (acronym: CINGLE-trial; Cochlear Implantation for siNGLE-sided deafness) is presented. The study protocol was published to help reduce publication bias and improve transparency, since others can check if all intended measurements have actually taken place. In the CINGLE-trial, 120 adult single-sided deaf patients (duration of deafness $>3$ months and maximum 10 years; pure tone average threshold (at $0.5,1,2,4 \mathrm{kHz}$ ) of the best ear: maximum $30 \mathrm{~dB} \mathrm{HL}$, and of the poor ear: minimum $70 \mathrm{~dB} \mathrm{HL}$ ) were included and randomized to treatment groups: cochlear implant (CI), trial period 'first BCD, then CROS', or trial period 'first CROS, then BCD'. After the trial periods, patients in the two latter groups chose with which treatment option they wished to continue: $\mathrm{BCD}, \mathrm{CROS}$, or no treatment. Outcomes of interest were speech perception in noise, sound localization, tinnitus and quality of life.

The short-term results of the CINGLE-trial were presented in Chapter 5. After 3 and 6 months of follow-up, the results showed that cochlear implantation led to improved speech perception in noise, depending on the test configuration. In the $\mathrm{S}_{0} \mathrm{~N}_{0}$ configuration, the CI group performed significantly better than the BCD and CROS groups, and also when 
compared to baseline. In the $\mathrm{S}_{\mathrm{pe}} \mathrm{N}_{\mathrm{be}}$ configuration, there was an advantage for all treatment groups compared to baseline. However, in the $\mathrm{S}_{\mathrm{be}} \mathrm{N}_{\mathrm{pe}}$ configuration, $\mathrm{BCD}$ and CROS groups performed worse, whereas the CI group improved. Sound localization improved in the CI group only. On both the Tinnitus Questionnaire and Tinnitus Handicap Inventory, tinnitus burden decreased in the CI group, whereas there was no significant change in the BCD and CROS groups compared to baseline. Moreover, three out of 28 patients reported total suppression of tinnitus after cochlear implantation. Patients with no tinnitus at baseline did not develop tinnitus after any intervention. In general, all treatment groups improved on disease-specific QoL.

An important strength of our trial is the random allocation of patients to intervention groups, ensuring equal distribution of patient characteristics across groups. Second, we compared CI to BCD and CROS in the same trial in a between-group analysis. Third, we had very few missing data. The most important limitation to consider is the limited generalizability of our results, because we included only a subset of patients with SSD. Only those who seeked help and met our in- and exclusion criteria were eligible for inclusion. Second, neither patients nor personnel were blinded for the allocated intervention; this is a well-known problem in nonpharmacological studies. ${ }^{7}$

\subsubsection{Part III: vocoder and pitch match experiments}

So far, it was unknown how this electrical stimulation sounds. In Chapter 6, we aimed to answer the question 'What does a CI sound like?'. Patients with SSD and a CI form a unique population, since they can compare the sound of their CI to simulations of the CI sound played to their non-implanted ear. We presented speech and music stimuli to the CI-ear of our SSD patients, and subsequently asked them to compare the stimuli to modified versions of these stimuli presented to their normal-hearing ear. We obtained a fairly good impression of what a CI may sound like for SSD patients. The average grade for similarity was 6.8 (out of 10) for speech stimuli and 6.3 for music stimuli. This knowledge may help to better inform 
and educate patients and family members about the sound of a CI.

We evaluated another aspect of the unique possibility of comparing the acoustic to the electric ear in Chapter 7. A pitch mismatch between the ears may negatively bear on the fusion of the signals from the two ears, which may limit auditory performance. Patients listened to two acoustic stimuli and we asked them to select the tone with the pitch closest to the pitch of one activated electrode contact, ultimately resulting in a "pitch match" per electrode contact. This matched pitch was compared to two references (the spiral ganglion map, and the default frequency allocation by manufacturer Cochlear Ltd.), and the difference was defined as "mismatch". There was no significant correlation between this mismatch and performance (consonant-vowel-consonant phoneme recognition score). We concluded that no changes in clinical fitting strategies were warranted.

In Chapter 8, a similar analysis was done, but now the purpose was to investigate if the type of electrode array is of influence on the electric-acoustic pitch match. For any given electrode contact, the mismatch was smaller for the CI512 (perimodiolar) electrode array than for the CI422 (lateral wall) electrode array. The results remained stable over time (mean interval between test sessions was 4.3 months), with no significant difference between two test sessions considering all electrode contacts. Neither group showed a significant correlation between the mismatch and phoneme recognition scores.

\subsubsection{Part IV: Quality of reporting}

In Part I of this thesis, two systematic reviews were presented. In Part II, we described the study protocol and the short-term results of a RCT. As a final part in this thesis, we described two studies in which we evaluated the quality of reporting of systematic reviews and RCTs in the field of otorhinolaryngology. Adequate reporting of systematic reviews and RCTs is important, because only then readers can access and understand all relevant and necessary information. 
In Chapter 9, our objective was to evaluate the quality of reporting of systematic reviews and meta-analyses and their abstracts in otorhinolaryngologic literature using the Preferred Reporting Items for Systematic reviews and Meta-Analyses (PRISMA) (for Abstracts) checklist. A similar analysis was described in Chapter 10, but now focusing on the quality of reporting of randomized controlled trials (RCTs) and their abstracts by the using Consolidated Standards of Reporting Trials (CONSORT) (for Abstracts) checklist. We found that the reporting of systematic reviews and RCTs in otorhinolaryngologic journals leaves room for improvement. The quality of reporting may improve if authors would adhere to the CONSORT and PRISMA checklists, and if these two statements would be endorsed by the editorial boards of journals in otorhinolaryngology.

\subsection{Future directions of research}

\subsubsection{Detailed analysis of trial period BCD and CROS}

In Chapter 5, the results of our RCT evaluating CI, BCD, CROS and no treatment were presented after 3 and 6 months follow-up. As described in the study protocol (Chapter 4), when randomized to the $\mathrm{BCD} / \mathrm{CROS}$ groups, patients could opt for $\mathrm{BCD}, \mathrm{CROS}$, or no treatment. The proportion of patients opting for a BCD after the trial period in our study (29.4\%) was on the low end of the range described in literature for patients opting for a BCD after a BCD trial period (32.0-69.6\%). ${ }^{8}$ This lower proportion opting for BCD may be explained by the cross-over design in our trial: patients also tested the CROS hearing aid. Moreover, the proportion of patients opting for a BCD could have been influenced by the type of BCD offered during the trial period; we noticed that the satisfaction with the newer Baha 5 Power was significantly higher than with the older Baha ${ }^{\circledR}$ BP110.

To perform a detailed analysis of the trial period, we administered disease-specific QoL questionnaires to all patients in the trial period. We aim to evaluate their experiences with BCD and CROS with these questionnaires, amongst other outcomes, in the near future. 
We hope to learn more about the satisfaction of patients with either device, and hopefully identify prognostic factors (e.g. duration of SSD, pure tone audiometry of better ear, transcranial attenuation ${ }^{9,10}$ ) that may help us to predict satisfaction with either device.

\subsubsection{Long-term results after cochlear implantation}

\section{Tinnitus}

In Chapter 5 we described that tinnitus burden decreased in the CI group, whereas there was no significant change in the BCD and CROS groups compared to baseline and compared to the group with no treatment. After cochlear implantation, three out of 28 patients patients reported total suppression of tinnitus. Patients with no tinnitus at baseline did not develop tinnitus after any intervention. For patients the tinnitus burden can be very incapacitating. Therefore, we hope to further analyze tinnitus burden in the future, using the Tinnitus Burden Questionnaire. For the CI group, we asked patients to complete this questionnaires with the CI switched on and off; we will be able to see if the suppressing effect of the CI on tinnitus is still present when the CI is switched off.

\section{Duration of CI use}

In Chapter 5, we described that the median duration of CI use per day during the first 6 months after fitting was 12.7 hours [range 2.0 - 16.3 hours], based on the electronic data logging of the speech processor. There were no non-users in the CI group during the first 6 months of follow-up and no serious adverse events occured. Later follow-up moments are not completed by all patients yet, but we know from the already completed 12- and 24-months follow-up moments that not all patients wore their CI the whole day, and some patients even did not wear their CI at all anymore. These patients indicated that the sound of the CI was distracting them from hearing with their better ear, and they felt hindered by the device. It will be interesting to see how device use develops with longer follow-up, because non-use may occur when follow-up time is longer. ${ }^{6,11}$ Median duration of CI use per day will remain 
an important factor to consider. If patients hardly use the device since they experience only little benefit, one should wonder if the necessary surgery and additional costs are justified.

Finally, in the near future we aim to analyze correlations between outcomes (also on true binaural tasks such as speech perception in noise and sound localization) and patient characteristics (e.g. duration of SSD, pure tone average of the better ear, etiology etc.). If any correlations exist, this may help us to predict outcomes and thereby improve counselling of patients.

\section{Cost utility analysis}

An important aspect of new medical interventions is its cost utility (cost/benefit ratio). Whether the costs of a new treatment option are reimbursed depends, amongst other factors, on the effectiveness of the new treatment: is society willing to pay (much) more for extra (or equal) benefit?

Associated with the costs of cochlear implantation are not only the device costs, but also costs for surgery, nursing and anaesthetic personnel and all costs for the rehabilitation phase (audiologists and speech and language therapists). These costs are much higher than the costs of the conventional treatment options; CROS hearing aids are only slightly more expensive than conventional hearing aids, and no surgery or rehabilitation phase is required. For the BCD, surgery is shorter and less invasive, and only a short rehabilitation phase is needed. Furthermore, in the CINGLE-trial participants note work- and healthcare-related costs in a cost diary which they keep monthly during the first two years of study participation (see Chapter 4).

In health economics, benefit is defined as the gain in utility. Utility is expressed as a number between 0 (death) and 1 (perfect health). In the CINGLE-trial, utility is measured with the TTO, EQ5D and HUI3 questionnaires.

Based on a cost utility analysis with data from the 1- or 2-year follow-up moment, we will be able to state the costs of additional gain in utility of the treatment options in the future. It is then up to society and the reimbursement agencies to decide if cochlear implan- 
tation should be reimbursed as a treatment option for SSD or not. A recent modelling study on patients with SSD found that cochlear implantation may be cost-effective compared with no intervention, but BCDs were unlikely to be cost-effective. ${ }^{12}$ Importantly, on a societal level, the costs of not providing a treatment should also be taken into account to make a balanced decision.

\subsubsection{Vocoder and pitch match experiments}

In Chapter 6, we aimed to answer the question “What does a CI sound like?". Based on the experiments described, we obtained a fairly good idea of what a CI sounds like. In cooperation with researchers from Arizona State Univerisity (Phoenix, AZ, USA) we developed several simulations that aimed to approximate the sound of a CI. Based on these succesfull first experiments, we created plans to approach the sound of a CI even better. These experiments are currently ongoing and will be analyzed in a later stage. First, we developed an automated user interface so the patient can listen to stimuli independent of the researcher, and they can listen to stimuli several times. At the end of the automated program that aims to approximate the sound of a CI, patients are offered several options to adjust the sound simulation so the similarity of the sound simulation approaches the sound of their CI even better. Simultaneously, the research group from Arizona also created newer simulations with which they reached a similarity to the CI sound of 8.8 on a scale of $1-10 .{ }^{13}$ The most common alterations to a clean signal were band-pass or low-pass filtering, spectral peak smearing, and F0 contour flattening. Even better counselling of CI candidates and their family members will be possible with these improved methods to approach the sound of a CI.

Based on the experiments described in Chapters 7 and 8, we concluded that there was no significant correlation between the defined place-to-frequency mismatch and performance. The results remained stable over time, with no significant difference between two test sessions considering all electrode contacts. The pitch mismatch was smaller for the perimodiolar electrode array than for the lateral wall electrode array. In the future we aim to correlate 
the place-to-frequency mismatch and the intracochlear position of the electrode contacts to true binaural performance tests (e.g. speech perception in noise, sound localization).

\subsubsection{Quality of reporting}

In Chapters 9 and 10, we described that the reporting of both systematic reviews and RCTs in otorhinolaryngologic journals leaves room for improvement. The quality of reporting may improve if authors would adhere to the CONSORT and PRISMA checklists, and if these two statements would be endorsed by the editorial boards of journals in otorhinolaryngology.

It would be interesting to repeat our analysis in 5-10 years and evaluate if the quality of reporting has increased, and evaluate if editorial boards recommend the use of reporting guidelines. 


\subsection{Conclusions}

There were no high level of evidence studies comparing BCD, CROS and CI for patients with SSD. Based on available literature, cochlear implantation seemed to be a promising treatment option, which needed further analysis in an RCT. Consequently, we developed an RCT in which these treatment options for patients with SSD were investigated.

The short-term results of this RCT showed that speech perception in noise improved in all configurations for the CI group. For the speech the BCD and CROS groups, speech perception in noise improved when speech was presented to the poor ear, but deteriorated when speech was presented to the better ear. Only in the CI group, sound localization improved and tinnitus burden/distress decreased. In general, all treatment options improved disease-specific QoL on most subscales of the used questionnaires.

Long-term objective and subjective outcomes will be presented in the future, as well as data about patient satisfaction derived from the duration of device use, and also adverse events will be monitored. Finally, a detailed analysis of costs of the treatments compared to (gained) utility will provide information to health care policy makers to decide whether cochlear implantation should be reimbursed as a treatment option for SSD.

In additional experiments in the CI group, we obtained a fairly good idea of what a CI sounds like, which may help the counselling of CI candidates and their family members. Futhermore, we concluded that there was no significant correlation between the defined place-to-frequency mismatch and performance. Therefore, the default frequency allocation table should not be altered.

Finally, we found that both the reporting of systematic reviews and of RCTs in otorhinolaryngologic journals leaves room for improvement. The quality of reporting would probably benefit if authors and editors adhere to reporting guidelines. 


\section{REFERENCES}

1. Kitterick PT, Smith SN, Lucas L. Hearing Instruments for Unilateral Severe-to-Profound Sensorineural Hearing Loss in Adults: A Systematic Review and Meta-Analysis. Ear Hear 2016;37(5):495-507.

2. Mertens G, De Bodt M, Van de Heyning P. Evaluation of Long-Term Cochlear Implant Use in Subjects With Acquired Unilateral Profound Hearing Loss: Focus on Binaural Auditory Outcomes. Ear Hear 2017;38(1):117-125.

3. Galvin JJ 3rd, Fu QJ, Wilkinson EP, Mills D, Hagan SC, Lupo JE, Padilla M, Shannon RV. Benefits of Cochlear Implantation for Single-Sided Deafness: Data From the House Clinic-University of Southern California-University of California, Los Angeles Clinical Trial. Ear Hear 2019;40(4):766-781.

4. Peter N, Liyanage N, Pfiffner F, Huber A, Kleinjung T. The Influence of Cochlear Implantation on Tinnitus in Patients with Single-Sided Deafness: A Systematic Review. Otolaryngol Head Neck Surg 2019;161(4):576-588.

5. Kurz A, Rak K, Hagen R, Ehrmann-Müller D. Evaluating the Decision for Cochlear Implantation in Individuals With Single-Sided Deafness (SSD); Implementing the SSD Consensus Protocol Into Clinical Routine. Otol Neurotol 2020;41(6):727-735.

6. Muigg F, Bliem HR, Kühn H, Seebacher J, Holzner B, Weichbold VW. Cochlear implantation in adults with single-sided deafness: generic and disease-specific long-term quality of life. Eur Arch Otorhinolaryngol 2020;277(3):695-704.

7. Boutron I, Tubach F, Giraudeau B, Ravaud P. Blinding was judged more difficult to achieve and maintain in nonpharmacologic than pharmacologic trials. J Clin Epidemiol 2004;57(6):543-550.

8. Wendrich AW, Kroese TE, Peters JPM, Cattani G, Grolman W. Systematic Review on the Trial Period for Bone Conduction Devices in Single-Sided Deafness: Rates and Reasons for Rejection. Otol Neurotol 2017;38(5):632-641. 
9. Stenfelt S. Bilateral fitting of BAHAs and BAHA fitted in unilateral deaf persons: acoustical aspects. Int J Audiol 2005;44(3):178-189.

10. Stenfelt S. Transcranial attenuation of bone-conducted sound when stimulation is at the mastoid and at the bone conduction hearing aid position. Otol Neurotol 2012;33(2):105114 .

11. Sullivan CB, Al-Qurayshi Z, Zhu V, Liu A, Dunn C, Gantz BJ, Hansen MR. Long-term audiologic outcomes after cochlear implantation for single-sided deafness. Laryngoscope 2020;130(7):1805-1811.

12. Ontario Health (Quality). Implantable devices for single-sided deafness and conductive or mixed hearing loss: a health technology assessment. Ont Health Technol Assess Ser (Internet) 2020;20(1):1-165. Available from: https://www.hqontario.ca/Evidenceto-Improve-Care/Health-Technology-Assessment/Reviews-And-Recommendations/ Implantable-Devices-for-Single-Sided-Deafness-and-Conductive-or-Mixed-HearingLoss, accessed 1 April 2020.

13. Dorman MF, Natale SC, Baxter L, Zeitler DM, Carlson ML, Lorens Artur, Skarzynski H, Peters JPM, Torres JH, Noble JH. Approximations to the Voice of a Cochlear Implant: Explorations With Single-Sided Deaf Listeners. Trends Hear 2020;24:2331216520920079. 


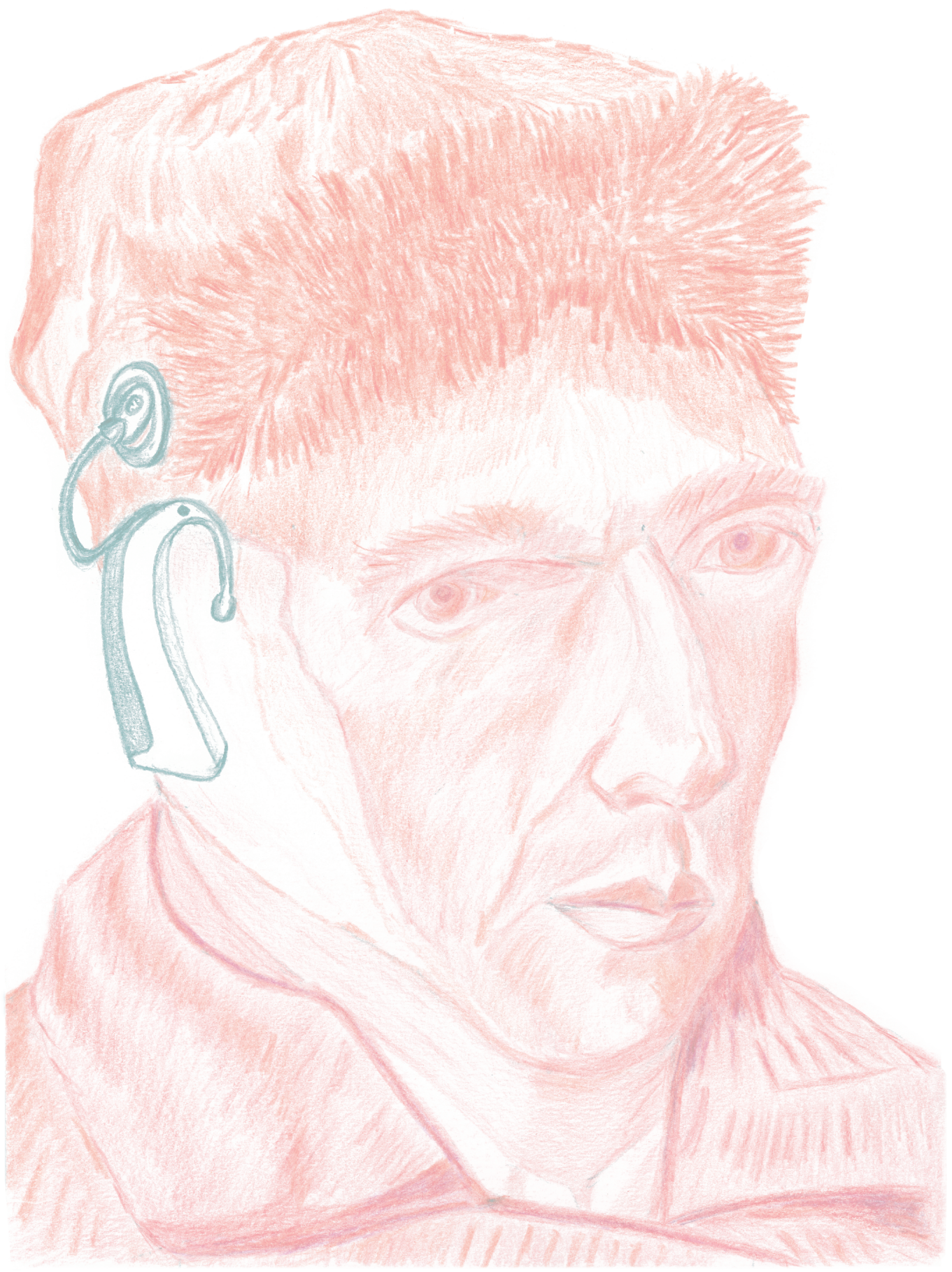




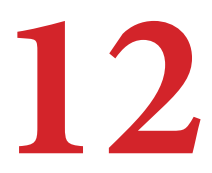

Samenvatting in het Nederlands 


\subsection{Deel I: Introductie en overzicht van literatuur}

Hoofdstuk 1 bevat een algemene inleiding op het onderwerp van dit proefschrift: het optimaliseren van de behandeling van eenzijdige doofheid. De werking van het gehoor wordt besproken, alsook het 'binauraal horen': horen met twee oren. Doordat we kunnen horen met twee oren, kunnen we geluiden lokaliseren en beter spraak verstaan in rumoer (ruis) dan met één oor. Er zijn drie effecten die bijdragen aan binauraal horen: squelch effect, summatieeffect en hoofdschaduweffect.

Bij eenzijdige doofheid kunnen patiënten geen gebruik maken van deze effecten, en dus verstaan zij spraak in ruis moeilijker en lokaliseren zij geluiden moeizamer. Bovendien ervaren eenzijdig dove patiënten in het dove oor vaak oorsuizen (tinnitus), wat mogelijk verklaard wordt door het gebrek aan signalen vanuit het slakkenhuis naar het brein. Als gevolg van deze problemen ervaren deze patiënten vaak een lagere kwaliteit van leven.

Er zijn twee huidige behandelingen voor eenzijdige doofheid: 1. Het CROS-toestel (Contralateral Routing of Sound hearing aid (CROS)) bestaat uit twee hoortoestellen: één hoortoestel aan de dove zijde brengt het geluid van die zijde naar het andere hoortoestel aan de goede zijde). 2. Het beengeleider hoortoestel (Bone Conduction Device (BCD)) zit met een schroef in de schedel aan de dove zijde. Het apparaat vangt geluiden aan de dove zijde op en geeft deze geluidstrillingen door aan de schedel, waardoor ook de vloeistof in het slakkenhuis aan de goede zijde gaat trillen. Het effect van een BCD kan gesimuleerd worden met een BCD op een strakke band. Zowel CROS als BCD brengen het geluid dus naar het goede slakkenhuis; er wordt geen signaal aangeboden aan het dove slakkenhuis.

In tegenstelling tot de twee huidige behandelingen biedt een cochleair implantaat (CI) een signaal aan het slakkenhuis aan de dove zijde aan. Geluid wordt door een processor omgezet in elektrische informatie en afgegeven aan een elektrode in het slakkenhuis. Hiermee wordt geluidsinformatie via de gehoorzenuw naar het brein gebracht en kunnen patiënten dus theoretisch weer 'horen met twee oren'. Wij verwachten dat zij dus beter spraak in ruis kunnen verstaan en beter geluiden kunnen lokaliseren. Bovendien hebben zij mogelijk, door 
de herstelde informatievoorziening aan de dove zijde, minder last van tinnitus. Met deze voordelen is hopelijk de kwaliteit van leven beter.

Om deze drie behandelingen goed te vergelijken, moet een vergelijkende studie zo min mogelijk risico op bias (een verstoring van uitkomsten door externe factoren, waardoor de gemeten resultaten niet de werkelijkheid representeren) hebben. Een gerandomiseerde studie waarbij patiënten in behandelgroepen worden ingedeeld door het lot, kan een groot aantal vormen van bias wegnemen. Daarom heeft dit type studie de voorkeur om behandelingen te vergelijken.

In Hoofdstuk 2 hebben we een literatuuroverzicht gemaakt van de reeds beschikbare studies naar de twee huidige behandelingen (CROS en BCD) van eenzijdige doofheid. We stelden criteria op waaraan de studies moesten voldoen om ze mee te nemen in onze beoordeling. De geschikte studies waren zeer divers van opzet, waardoor het niet mogelijk was om de resultaten samen te voegen. Daarom hebben we de resultaten per uitkomstmaat beschreven. De studies die geschikt waren voor beoordeling bleken allemaal van matige kwaliteit en bevatten een gemiddeld tot hoog risico op verschillende vormen van bias. Met inachtneming van deze beperkingen, beschreven de studies over het algemeen dat er geen voordeel van BCD en CROS bestond bij het spraakverstaan in ruis of geluidslokalisatie vergeleken met de situatie zonder deze hulpmiddelen. Ook verbeterde de kwaliteit van leven niet; wel werd de 'kwaliteit van horen', gemeten met enkele vragenlijsten, beter. We concludeerden dat er studies naar de toepassing van BCD en CROS bij patiënten met eenzijdige doofheid met een betere kwaliteit moeten plaatsvinden.

Een soortgelijk literatuuroverzicht schreven we in Hoofdstuk 3 over de toepassing van CI's bij eenzijdige dove patiënten. Met eenzelfde methode beoordeelden we artikelen; ook nu konden we de resultaten van verschillende studies niet samenvoegen. Wederom bleek de kwaliteit van de studies matig door verschillende vormen van bias. De geïncludeerde studies laten een 
voordeel van CI zien bij geluidslokalisatie in vergelijking met geen behandeling. Ook is er minder tinnituslast en was de kwaliteit van leven verbeterd. Het effect op spraakverstaan in ruis was afhankelijk van in welke configuratie de test werd afgenomen (spraak aangeboden richting goede oor en ruis richting dove oor, of vice versa). Ook nu concludeerden we dat er studies zouden moeten plaatsvinden met een hogere kwaliteit om de effecten van CI bij eenzijdig dove patiënten te beoordelen.

\subsection{Deel II: CINGLE-studie}

Hiertoe hebben we een studie-opzet gemaakt, waarin we een gerandomiseerde vergelijking maken tussen de verschillende behandelingen van eenzijdige doofheid. In Hoofdstuk 4 staat het protocol van deze studie (CINGLE-studie, Cochlear Implantation for siNGLE-sided deafness). We beschrijven dat we 120 volwassenen willen includeren, bij wie de eenzijdige doofheid tussen de 3 maanden en 10 jaar bestaat, en bij wie de gemiddelde gehoordrempel in het goede oor maximaal $30 \mathrm{~dB}$ is en in het slechte oor minimaal $70 \mathrm{~dB}$ is. Zij werden gerandomiseerd naar de groepen: 1. CI, 2. proefperiode met eerst BCD, dan CROS, en 3. proefperiode met eerst CROS, dan BCD. In de groepen 2 en 3 konden de patiënten na twee keer zes weken beide toestellen op proef (BCD op hoofdband en CROS-toestel) te hebben geprobeerd, besluiten welke optie zij het fijnst vonden: BCD (welke dan werd geïmplanteerd), CROS-toestel, of geen behandeling. Hiermee ontstonden dus in totaal 4 groepen (CI, BCD, CROS en geen behandeling) waarbij we geïnteresseerd waren in spraakverstaan in ruis, geluidslokalisatie, verminderen van tinnituslast (vragenlijsten) en kwaliteit van leven/horen (vragenlijsten).

De korte termijn resultaten van de CINGLE-studie worden beschreven in Hoofdstuk 5. We hebben de effecten gemeten 3 en 6 maanden na start van de behandelingen (de totale followup termijn is 5 jaar). Ook wij meten dat het voordeel bij spraakverstaan in ruis afhankelijk 
is van de configuratie waarin gemeten wordt. Als spraak en ruis beiden van voren worden aangeboden, presteert de CI-groep beter dan de BCD- en CROS-groep, en ook beter dan de patiënten zonder behandeling. Als de spraak richting het dove oor en de ruis richting het goede oor wordt aangeboden, bieden alle behandelingen voordeel. Echter, als de spraak richting het goede oor en de ruis richting het dove oor wordt aangeboden, verslechtert de prestatie van de BCD- en CROS-groep, terwijl de prestatie van de CI-groep zelfs dan beter wordt. Geluidslokalisatie wordt alleen beter na behandeling met een CI. Op twee tinnitusvragenlijsten blijkt er in de CI-groep minder last van tinnitus te bestaan dan vóór de behandeling, terwijl er geen verschil gevonden wordt voor de BCD- en CROS-groep en de patiënten zonder behandeling. Over het algemeen verbeterde de kwaliteit van leven bij alle drie de behandelgroepen, en bleef deze onveranderd bij de patiënten zonder behandeling.

Een belangrijke kracht van de CINGLE-studie is de gerandomiseerde indeling van patiënten naar groepen, want hiermee worden bekende en onbekende factoren die mogelijk van invloed zijn op de uitkomsten gelijk verdeeld over de behandelingen. Verder biedt onze studie de mogelijkheid om de behandelingen onderling te vergelijken, in plaats van slechts één (of twee) behandelingen te onderzoeken. Een nadeel van onze studie is dat we patiënten onderzocht hebben die mogelijke geen representatieve steekproef zijn van alle patiënten met eenzijdige doofheid. Immers, alleen de mensen met klachten zochten onze hulp. Daarnaast onderzochten we alleen de mensen die voldeden aan de criteria om mee te 'mogen' doen aan de studie. We kunnen onze resultaten dus niet extrapoleren naar alle eenzijdig dove patiënten.

\subsection{Deel III: Vocoder-experiment en toonhoogtevergelijkingsexperimenten}

In dit deel van het proefschrift hebben we aanvullende experimenten gedaan bij de CI-patiënten uit de CINGLE-studie. Zij vormen een unieke populatie, want hun niet-geïmplanteerde oor heeft een goed gehoor; zij kunnen dus het CI-geluid vergelijken met geluiden in hun goede oor. 
In Hoofdstuk 6 poogden we uit te zoeken hoe een cochleair implantaat klinkt. We hebben spraak- en muziekfragmenten aangeboden aan het CI-oor van eenzijdig dove CI-patiënten (goede oor gemaskeerd), en vervolgens lieten we hen bewerkte versies horen aan het goede oor (CI uit). We vroegen hen een cijfer voor de overeenkomst tussen beide geluiden te geven.

Het gemiddelde cijfer voor overeenkomst bij de spraakfragmenten was 6,8/10 en voor muziekfragmenten $6,3 / 10$. Hiermee hebben we een redelijk idee van hoe een CI klinkt, wat kan helpen bij de voorlichting over cochleaire implantatie aan CI-kandidaten en familieleden.

Hoofdstuk 7 beschrijft een experiment waarbij CI-patiënten gevraagd werd om de toonhoogte van één elektrisch gestimuleerde electrode in hun CI-oor te vergelijken met een akoestisch aangeboden toon aan hun goede oor. Als de waargenomen toonhoogte tussen de oren verschilt, kan dit mogelijk leiden tot een slechtere fusie in het brein, wat mogelijk de prestatie belemmert. Patiënten luisterden naar twee akoestische tonen die steeds meer op elkaar leken en kozen daarbij steeds de toon welke qua toonhoogte het meest in de buurt kwam bij de toonhoogte die zij hoorden bij de geactiveerde electrode in hun CI-oor. De toon die uiteindelijk het meest paste noemden we de "match". Vervolgens werd, na positiebepaling van de electrode middels CT-scans, gekeken of de gematchte toon op de te verwachten plek lag, en dus overeen kwam met de normaalhorende zijde.

Er bleek een verschil te bestaan tussen de waargenomen toonhoogtes en de referenties ("mismatch"). Echter, deze mismatch was niet gecorreleerd met de prestatie op een spraakverstaan-test. Mogelijk past het brein zich al snel aan de vernieuwde input aan. De afwezige correlatie met spraakverstaan leidde er toe dat wij concludeerden dat de standaard frequentie-indeling van CI's niet aangepast zou moeten worden.

Een vervolg op deze experimenten wordt besproken in Hoofdstuk 8. We wilden nu 
onderzoeken of er een verschil in mismatch is tussen CI-patiënten met een perimodiolaire electrode (vlakbij de kern van het slakkenhuis) versus CI-patiënten met een elektrode die 'in de buitenbocht' van het slakkenhuis ligt. De mismatch voor de perimodiolaire electrode bleek kleiner. Ook wilden we onderzoeken of de resultaten veranderden na enige tijd (gemiddeld 4,3 maanden tussen de metingen). De resultaten bleven stabiel over deze periode. Wederom was er geen correlatie tussen de mismatch en prestatie.

\subsection{Deel IV: Kwaliteit van rapporteren}

We hebben in dit proefschrift twee literatuuroverzichten (systematische reviews, hoofdstuk 2 en 3) opgenomen, alsook een gerandomiseerde studie beschreven (hoofdstuk 5). Lezers kunnen de resultaten van deze studies alleen begrijpen en goed interpreteren als de studies volledig en adequaat gerapporteerd zijn. Om auteurs hierbij te helpen zijn er richtlijnen gepubliceerd welke adequaat rapporteren faciliteren. In dit laatste deel maakten we een overzicht van hoe de kwaliteit van rapporteren van deze studies is binnen de KNO-literatuur.

In Hoofdstuk 9 hebben we de kwaliteit van rapporteren van systematische reviews binnen de KNO beoordeeld met behulp van de PRISMA-checklist (Preferred Reporting Items for Systematic reviews and Meta-Analyses). Een soortgelijke beoordeling maakten we in Hoofdstuk 10 over de kwaliteit van rapporteren van gerandomiseerde studies met behulp van de CONSORT-checklist (Consolidated Standards of Reporting Trials).

We concludeerden in beide hoofdstukken dat de kwaliteit van rapporteren van beide studietypes binnen de KNO niet optimaal is. We adviseerden dat auteurs zouden moeten rapporteren volgens de richtlijnen en dat KNO-tijdschriften dit ook zouden eisen bij nieuw aangeboden artikelen. 


\subsection{Deel V: Discussie}

Hoofdstuk 11 is een samenvattende discussie van de voorgaande hoofdstukken uit het proefschrift, zoals ook dit hoofdstuk een samenvatting is. Verder wordt er aandacht besteed aan toekomstige onderzoeken die volgen na de opgedane kennis uit dit proefschrift. Zo zullen we een nadere analyse uitvoeren van de proefperiode van de CINGLE-patiënten die BCD en CROS geprobeerd hebben, om hopelijk te achterhalen of er factoren zijn die een succesvolle proef kunnen voorspellen. Ook zal er extra aandacht besteed worden aan de vermindering van tinnituslast die we in de CI-groep gemeten hebben. Naarmate de follow-up van de CINGLEpatiënten langer wordt, zullen we ook kunnen registreren hoe lang patiënten tevreden blijven met hun behandeling. Inmiddels weten we dat niet alle CI-patiënten hun CI de gehele dag dragen. Ook zal er een kosteneffectiviteitsanalyse volgen: weegt de gezondheidswinst op tegen de (meer)kosten van de nieuwe behandeling? Tot slot gaan we ook verder met de experimenten die alleen in deze unieke populatie mogelijk zijn: we willen nog beter te weten komen hoe een CI klinkt. Daarvoor gaan we met een geautomatiseerde interface en meer opties om geluidsimulaties te maken op zoek naar een nog betere overeenkomst tussen het CI-geluid en onze simulaties. Ook willen we de eerder gemeten mismatch nog correleren aan uitkomstmaten die binauraal horen meten (spraakverstaan in ruis, geluidslokalisatie) en kunnen we onderzoeken of er een verband bestaat tussen de intracochleaire electrodepositie op CT-scans en deze uitkomstmaten.

\subsection{Conclusies}

In de literatuur bestonden nog geen studies van goede kwaliteit die de behandelingen (CROS, BCD, CI) voor eenzijdige doofheid vergelijken. Gebaseerd op de beschikbare literatuur leek CI een veelbelovende behandeling, wat nader onderzocht zou moeten worden in een gerandomiseerde studie.

Hierop hebben een gerandomiseerde studie ontworpen waarin de behandelingen 
voor eenzijdige doofheid werden onderzocht. We hebben de korte termijn resultaten beschreven. Voor de CI-groep verbeterde het spraakverstaan in ruis in alle configuraties. Voor de BCD- en CROS groep verbeterde het spraakverstaan in ruis wanneer spraak richting het dove oor werd aangeboden (en ruis richting het goede oor), echter verslechterde het spraakverstaan in ruis in de omgekeerde configuratie. Alleen in de CI-groep verbeterde de geluidslokalisatie en was er minder tinnituslast. Over het algemeen was er een betere kwaliteit van leven voor alle behandelgroepen.

In de toekomst zullen we de lange termijn resultaten presenteren, en volgen er nadere analyses naar onder andere gebruiksduur, tinnituslast en kosteneffectiviteit.

Patiënten met een CI en een niet-geïmplanteerd oor met een goed gehoor bieden de unieke mogelijkheid om te weten te komen hoe een CI klinkt. We hebben voor spraaken muziekfragmenten een redelijk idee hoe een CI klinkt. In de toekomst hopen we met betere simulaties nog beter te weten hoe een CI klinkt. In experimenten waarbij CI-patiënten de toonhoogte van een geactiveerde electrode van het CI vergeleken met de toonhoogte van akoestisch aangeboden tonen aan het goede oor, bleek dat er een mismatch tussen beide oren bestond. Deze mismatch was echter niet van invloed was op de prestatie op een spraakverstaan-test, en daarom zou de standaard frequentie-indeling van CI's niet moeten wijzigen.

Tot slot concludeerden we dat de kwaliteit van rapporteren van literatuuroverzichten en gerandomiseerde studies binnen de KNO suboptimaal is. Teneinde de kwaliteit van rapporteren te verbeteren, is ons advies aan auteurs om te rapporteren volgens de opgestelde richtlijnen en het advies aan KNO-tijdschriften is om hierop te controleren. 


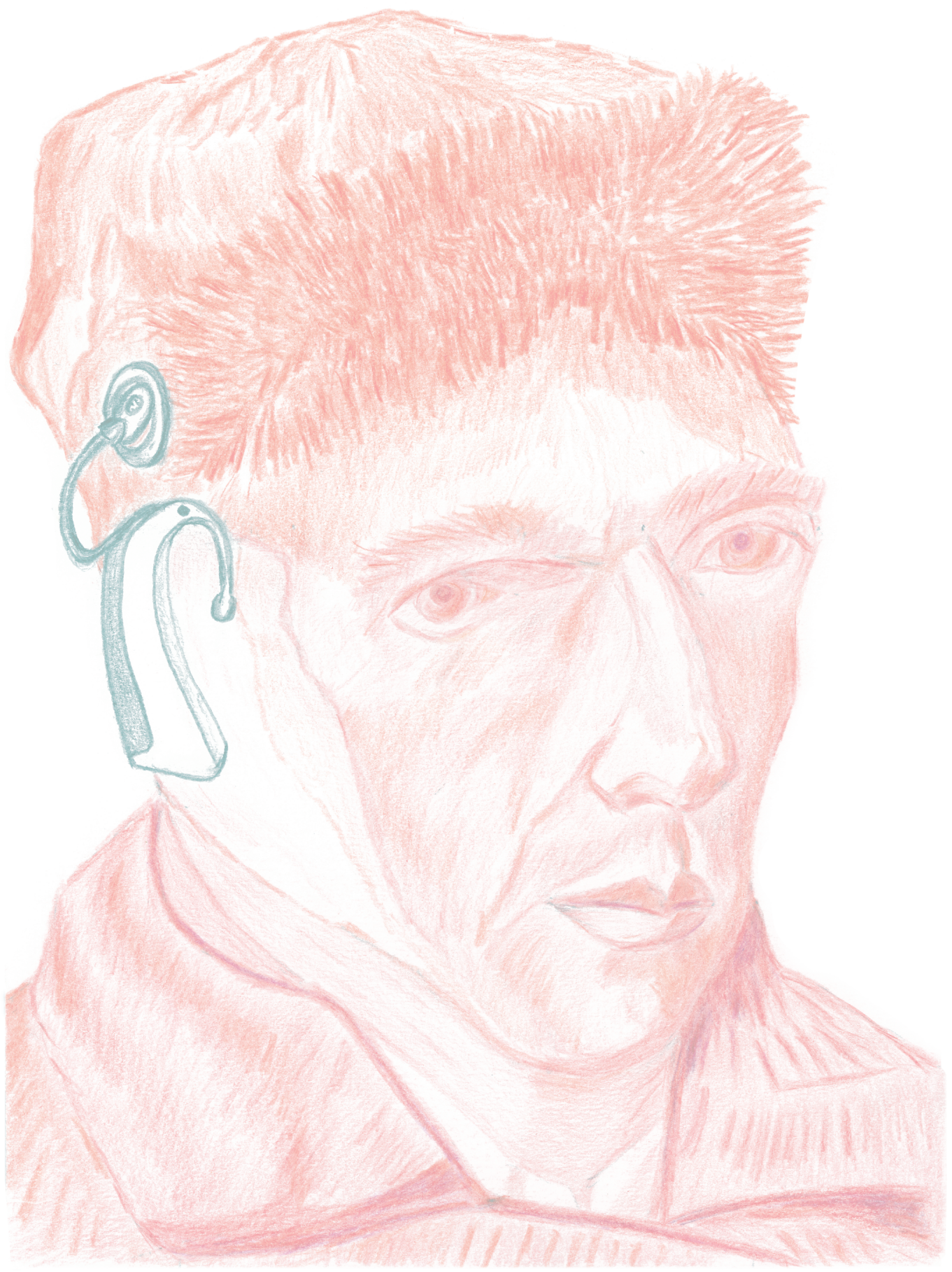


Appendices 


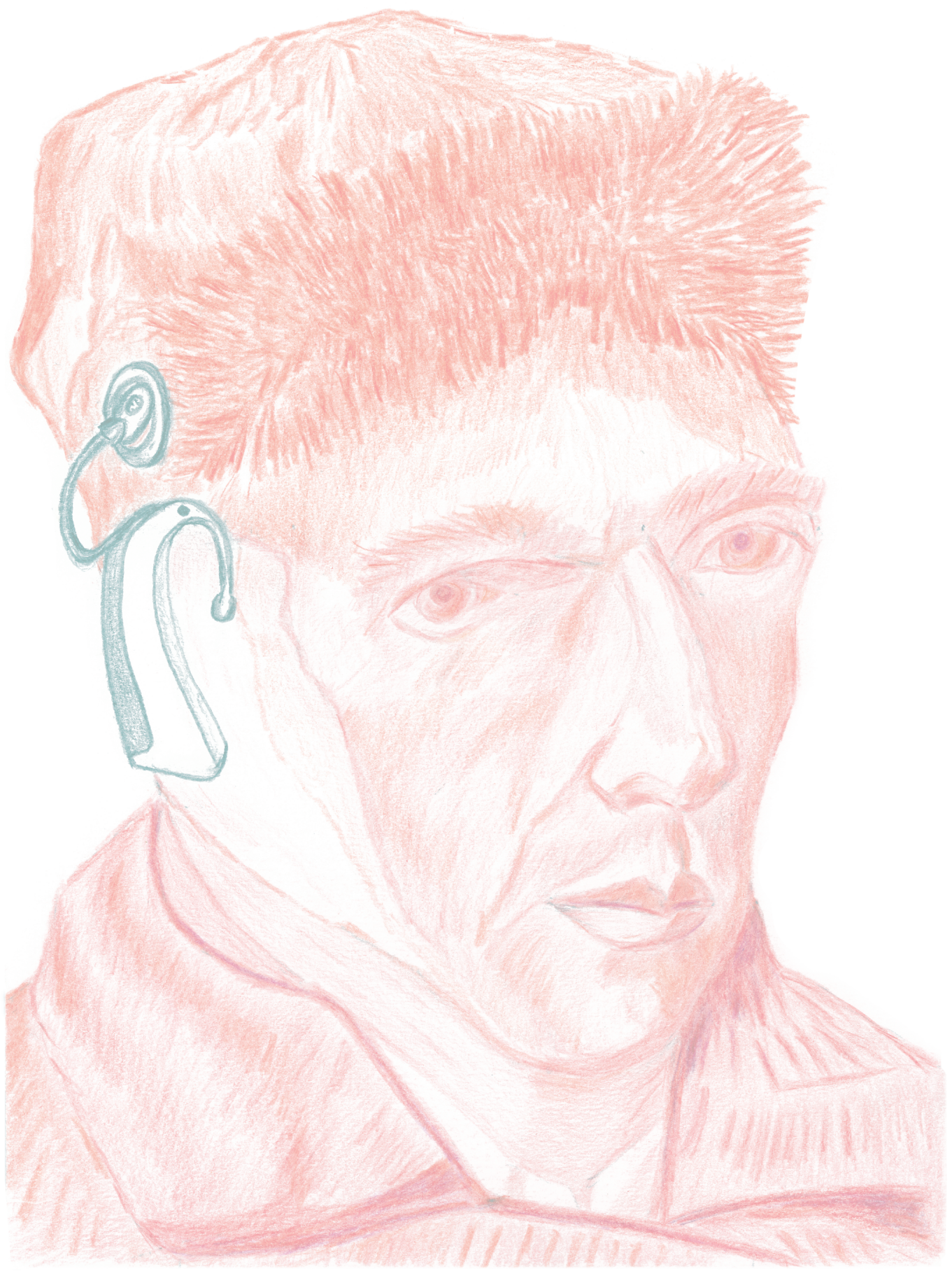




\section{List of Abbreviations}




\section{LIST OF ABBREVIATIONS}

APHAB

AHL

BAHA

$\operatorname{BCD}(\mathrm{s})$

$\mathrm{BKB}$

CDSR

CI

CIC

CINAHL

CINGLE-trial

CoD

CONSORT

CROS(S)

CVC

DAI

$\mathrm{dB}(\mathrm{HL})(\mathrm{SPL})(\mathrm{SNR})$
Abbreviated Profile for Hearing Aid Benefit, with subscales: EC:

ease of communication, RV: listening under reverberant conditions, BN: listening in background noise, AS: aversiveness of sounds

Asymmetric Hearing Loss

Bone Anchored Hearing Aid

Bone Conduction Device(s)

Bamford-Kowal-Bench speech test

Cochrane Database of Systematic Reviews

Cochlear Implant

Completely-in-the-canal hearing aid

Cumulative Index to Nursing and Allied Health Literature

Cochlear Implantation for siNGLE-sided deafness trial

Classification of deafness

Consolidated Standards of Reporting Trials

Contralateral Routing of Sound (System) hearing aid

Consonant-Vowel-Consonant

Direct Acoustic Input

decibel (Hearing Loss) (Sound Pressure Level) (signal-to-noise ratio)

DoD Duration of Deafness

DoE Directness of Evidence

Dmic Directional microphone

ENT Ear Nose Throat

EQ5D Euro-QoL 5D questionnaire

FU 
GBI

GHABP

HA

HADS

HINT

HL

HRCT

HSM

HUI3

$\mathrm{Hz}$

IC

ICMJE

ICUR

ILD

IOI-HA

IRB

ISTS

ITD

LIST

LoE

MA

$\mathrm{NH}$

$\mathrm{OlSa}$

PCS

Pt(s)

PTA $_{(\text {be) }}$
Glasgow Benefit Inventory

Glasgow Hearing Aid Benefit Profile

Hearing aid

Hospital Anxiety Distress Scale

Hearing In Noise Test

Hearing Loss

High Resolution Computed Tomography scan

Hochmair-Schulz-Moser sentence test (German)

Health Utilities Index 3 questionnaire

Hertz

Informed Consent

International Committee of Medical Journal Editors

Incremental Cost Utility Ratio

Interaural Level Differences

International Outcome Inventory for Hearing Aids

Institutional Review Board (in Dutch: Medisch Ethische

Toetsingscommissie)

International Speech Test Signal

Interaural Time Differences

Leuven Intelligibility Sentence Test

Level of Evidence

Meta-Analysis

Normal-hearing

Oldenburger sentence test (German)

Prospective Case Series

Patient(s)

Pure Tone Average (of the better ear) of 500, 1000, 2000 and $4000 \mathrm{~Hz}$ 
PRISMA

QoL

QUORUM

RCT

RCS

RoB

$\mathrm{S}_{0} \mathrm{~N}_{0}$

SAINT

$\mathrm{S}_{\mathrm{be}} \mathrm{N}_{\mathrm{pe}}$

SD

$\mathrm{S}_{\mathrm{pe}} \mathrm{N}_{\text {be }}$

SR

SRTn

SPIRIT

SSD

SSDq

SSQ

TBQ

THI

TQ

TRQ

TTO
Preferred Reporting Items for Systematic Reviews and Meta-

Analyses

Quality of Life

QUality Of Reporting Of Meta-analyses

Randomized Controlled Trial

Retrospective Case Series

Risk of Bias

Speech and Noise from 0 degrees azimuth (front), where 0 can

be any angle

Source Azimuth Identification in Noise Test

Speech from the side of the better ear, Noise from the side of the

poor ear

Standard Deviation

Speech from the side of the poor ear, Noise from the side of the

better ear

Systematic Review

Speech Reception Threshold in noise

Standard Protocol Items: Recommendations for Interventional

Trials

Single-Sided Deafness

Single-Sided Deafness questionnaire

Speech, Spatial and Qualities of hearing scale

Tinnitus Burden Questionnaire

Tinnitus Handicap Inventory

Tinnitus Questionnaire

Tinnitus Reaction Questionnaire

Time Trade Off questionnaire 
UMC Utrecht

U-STARR

VAS
University Medical Center Utrecht

Utrecht Sentence Test with Adaptive Randomized Roving levels

Visual Analogue Scale 


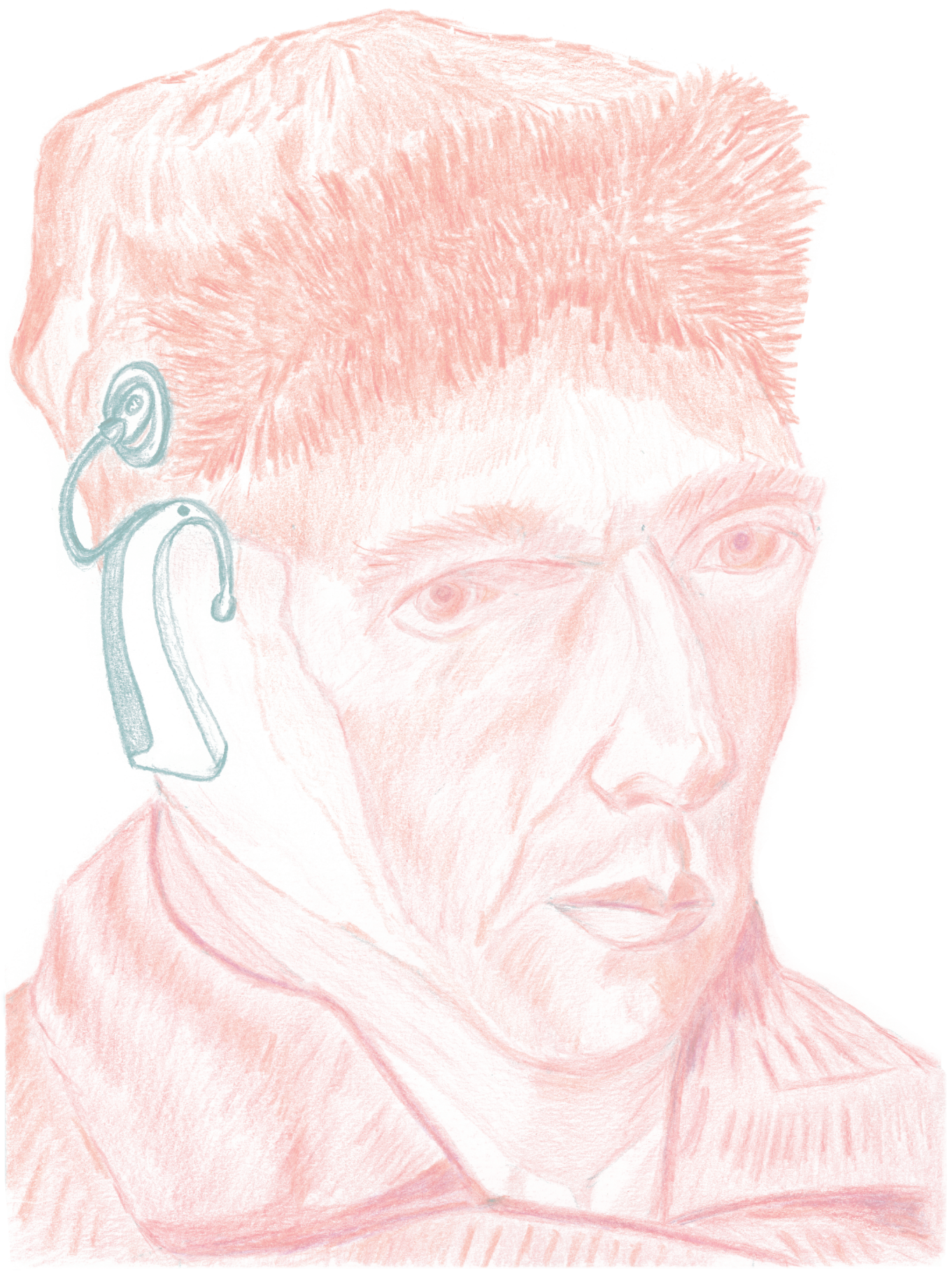




\section{List of Publications}




\section{LIST OF PUBLICATIONS (in chronological order)}

1. MF Dorman, SC Natale, L Baxter, DM Zeitler, ML Carlson, A Lorens, H Skarzynski, JPM Peters, JH Torres, JH Noble. Approximations to the Voice of a Cochlear Implant: Explorations With Single-Sided Deaf Listeners. Trends in Hearing 24, 2020;24:2331216520920079.

2. JPM Peters, PMW van Kempen, SMM Robijn, HGXM Thomeer. Angina Bullosa Hemorrhagica: Post-traumatic Swelling in the Oral Cavity - A Case Report. Journal of Advanced Oral Research 2020;11(1):97-100.

3. PMW van Kempen, JPM Peters, HGXM Thomeer. Een vrouw met een zwelling in de mond. Ned Tijdschr Geneeskd 2019;163. pii: D3837.

4. JAA van Heteren, I van Beurden, JPM Peters, AL Smit, I Stegeman. Trial registration, publication rate and characteristics in the research field of otology: A cross-sectional study. PloS One 2019;14(7).

5. Chapter 8

JPM Peters, E Bennink, GA van Zanten. Comparison of place-versus-pitch mismatch between a Perimodiolar and Lateral Wall Cochlear implant electrode Array in patients with single-sided deafness and a Cochlear implant. Audiology \& Neurotology 2019;24(1):38-48.

6. Chapter 6

JPM Peters, AW Wendrich, RHM Van Eijl, KS Rhebergen, H Versnel, W. Grolman. The Sound of a Cochlear Implant Investigated in Patients With Single-Sided Deafness and a Cochlear Implant. Otology \& Neurotology 2018;39(6):707-714.

7. A Bezdjian, SFL Klis, JPM Peters, W Grolman, I Stegeman. Quality of reporting of otorhinolaryngology articles using animal models with the ARRIVE statement. Laboratory Animals 2018;52(1):79-87.

8. JPM Peters, I Stegeman, W Grolman, L Hooft. The risk of bias in randomized controlled trials in otorhinolaryngology: hardly any improvement since 1950. BMC Ear, 
Nose and Throat Disorders 2017;17(1):3.

9. E Bennink, JPM Peters, AW Wendrich, E Vonken, GA van Zanten, MA Viergever. Automatic localization of cochlear implant electrode contacts in CT. Ear and Hearing 2017;38(6):e376-e384.

10. AW Wendrich, TE Kroese, JPM Peters, G Cattani, W Grolman. Systematic review on the trial period for bone conduction devices in single-sided deafness: rates and reasons for rejection. Otology \& Neurotology 2017;38(5):632-641.

11. M Hendriksma, MHMA Joosten, JPM Peters, W Grolman, I Stegeman. Evaluation of the quality of reporting of observational studies in otorhinolaryngology-based on the STROBE statement. PLoS One 2017;12(1).

\section{2. $\underline{\text { Chapter } 7}$}

JPM Peters, E Bennink, W Grolman, GA van Zanten. Electro-acoustic pitch matching experiments in patients with single-sided deafness and a cochlear implant: Is there a need for adjustment of the default frequency allocation tables? Hearing Research 2016;342:124-133.

13. SA Lyons, T Su, LET Vissers, JPM Peters, AL Smit, W Grolman. Fascia compared to one-piece composite cartilage-perichondrium grafting for tympanoplasty. Laryngoscope 2016;126(7):1662-1670.

14. JPM Peters, GGJ Ramakers, AL Smit, W Grolman. Cochlear implantation in children with unilateral hearing loss: A systematic review. Laryngoscope 2016;126(3):713-721.

\section{Chapter 4}

JPM Peters, A van Zon, AL Smit, GA van Zanten, GA de Wit, I Stegeman, W Grolman. CINGLE-trial: cochlear implantation for siNGLE-sided deafness, a randomised controlled trial and economic evaluation. BMC Ear, Nose and Throat Disorders 2015;15(1).

\section{Chapter 3}

A Van Zon, JPM Peters, I Stegeman, AL Smit, W Grolman. Cochlear implantation for patients with single-sided deafness or asymmetrical hearing loss: a systematic review of the evidence. Otology \& Neurotology 2015;36(2):209-219. 
17. Chapter 9

JPM Peters, L Hooft, W Grolman, I Stegeman. Reporting quality of systematic reviews and meta-analyses of otorhinolaryngologic articles based on the PRISMA statement. PLoS One 2015;10(8).

18. Chapter 10

JPM Peters, L Hooft, W Grolman, I Stegeman. Assessment of the quality of reporting of randomised controlled trials in otorhinolaryngologic literature-adherence to the CONSORT statement. PLoS One 2015;10(3).

19. Chapter 2

JPM Peters, AL Smit, I Stegeman, W Grolman. Bone conduction devices and contralateral routing of sound systems in single-sided deafness. Laryngoscope 2015;125(1):218226.

20. DMA Kamalski, JPM Peters, T de Boorder, SFL Klis, W Grolman. Influence of laser-assisted cochleostomy on acoustically evoked compound action potentials in the guinea pig. Otology \& Neurotology 2014;35(8):1306-1311.

21. DMA Kamalski, JPM Peters, T de Boorder, F Trabalzini, SFL Klis, W. Grolman. Effect of KTP laser cochleostomy on morphology in the guinea pig inner ear. ORL 2014;76(2):7075.

22. JPM Peters, W Kramer. Traumatische dwarslaesies bij kinderen; vroege en late gevolgen. Ned Tijdschr Geneeskd 2013;157(27):A5788. 



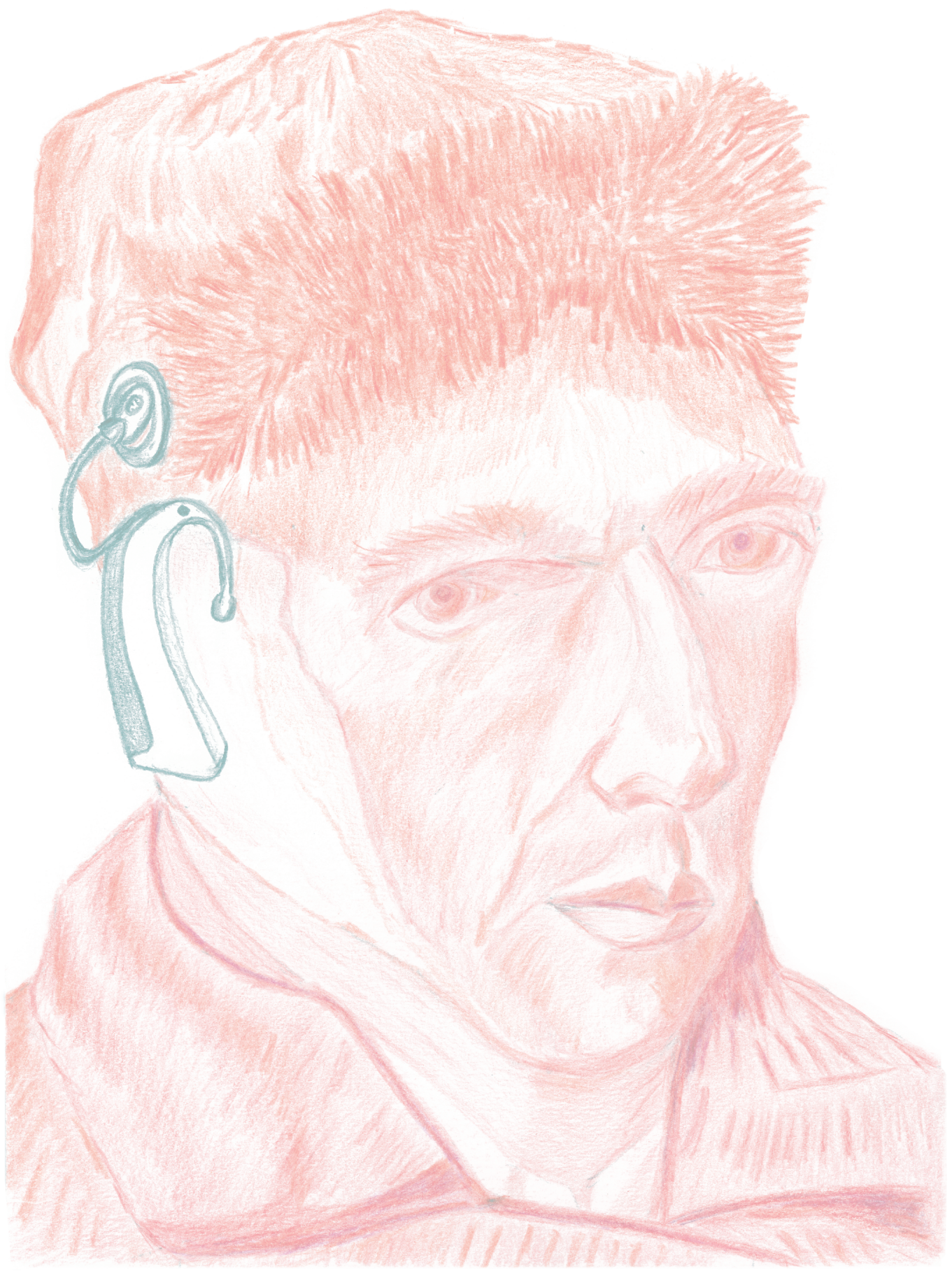




\section{PhD Portfolio}




\section{PhD PORTFOLIO - GRADUATE SCHOOL OF LIFE SCIENCES (GSLS)}

Name:

H.P.M. (Jeroen) Peters

PhD programme: $\quad$ Clinical and Experimental Neuroscience

\section{Educational programme}

\section{General courses}

2013, 2017 BROK-course, and re-registration

2014

Introduction to Brain Center Rudolf Magnus (BCRM)

BCRM Summerschool, Apeldoorn

- Workshop Time management

- Workshop Tutoring Master students

- Workshop Grant writing

- Workshop What is important for a scientific career

- Workshop Flirten - establishing a network

- Workshop How to make an effective poster presentation

- Workshop Scientific writing

- Workshop Blik-opener: career orientation

2016

ONWAR retreat, Woudschoten; poster presentation

1.0

Subtotal

\section{Theoretical / disciplinary courses}

2014

Workshop Statistical course, UMC Utrecht 
Courses of other GS-LS PhD programmes and/or attended external courses or meetings

2014

2014

2014

2015

2016

2016

2016

2016

2016

2018
Research Day Division of Surgical Specialties; poster presentation 0.3

Nederlandse Vereniging voor Audiologie, Utrecht; oral

0.3 presentation

Cochlear Science and Research Seminar,

Toulouse, France; oral presentation

Basis Kwalificatie Onderwijs (BKO), UMC Utrecht

Mid-Winter Meeting Association of Research in

1.2

Otolaryngology, San Diego, CA, USA; two poster presentations

0.3

presentation

Stichting Experimenteel Onderzoek Heelkundige

0.3

Specialismen (SEOHS), Utrecht; oral presentation

0.6

Cochlear Science and Research Seminar,

London, UK; oral presentation

0.9

Cochlear Implants Congress 2018, Antwerp, Belgium; oral presentation

2014 - 2020, 2x/yr Nederlandse Vereniging voor KNO-Heelkunde, Nieuwegein; in total three oral presentations

2016 - current Residency Otorhinolaryngology

Subtotal

14.1

TOTAL NUMBER OF CREDITS (EC) =

29.1 


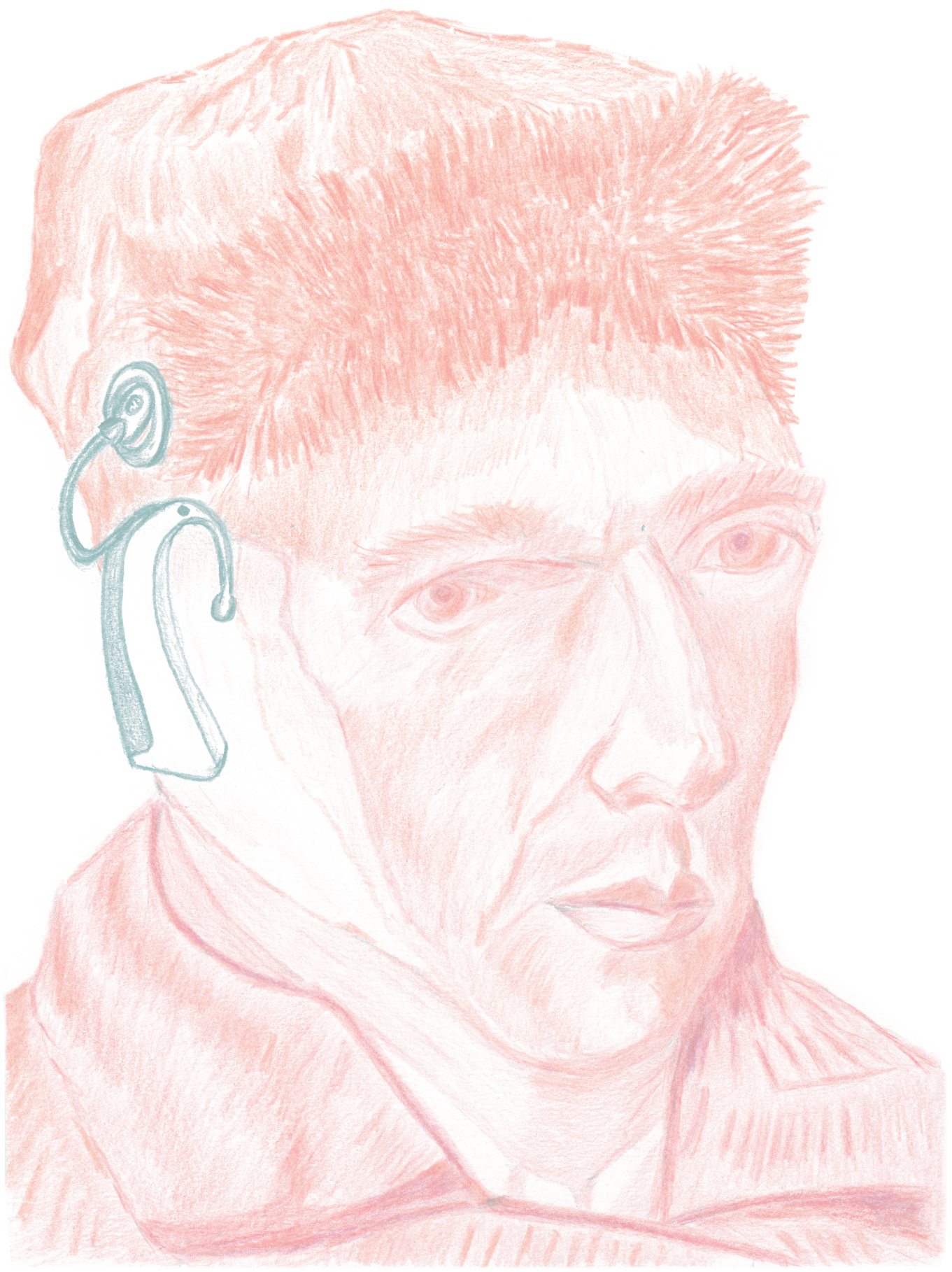




\section{Acknowledgements | Dankwoord}




\section{ACKNOWLEDGEMENTS | DANKWOORD}

Op deze plek wil ik alle mensen bedanken die - op welke manier dan ook - hebben bijgedragen aan het tot stand komen van dit proefschrift. Met name wil ik bedanken:

Alle deelnemers aan de CINGLE-studie, want zonder jullie hulp was dit onderzoek niet mogelijk geweest.

Prof. dr. Stokroos, beste Robert, bedankt voor je begeleiding bij dit project. Ik weet nog goed dat ik mijn eerste promotie-overleg met je had, vlak nadat je in Utrecht kwam werken. Jouw heldere blik op het gehele project ('een proeve van bekwaamheid') en het aanwakkeren van mijn intrinsieke motivatie ('de enige motivatie') hebben geholpen om dit proefschrift te maken tot wat het geworden is.

Prof. dr. Grolman, beste Wilko, bedankt voor de kans die je me bood tijdens mijn wetenschapsstage in het laatste jaar van mijn studie. Naast het afronden van het dierexperimenteel onderzoek vertrouwde je me ook het opzetten van een studieprotocol naar de behandelingen van eenzijdige doofheid toe. Ik herinner me goed de discussies die we voerden over de opzet van de CINGLE-studie in Utrecht, Amsterdam en in het vliegtuig naar Toulouse.

Dr. Smit, beste Diane, bedankt voor het begeleiden van het CINGLE-project vanaf de start. In het begin hebben we veel nagedacht over de opzet van de studie en over hoe we de groepen het best konden vergelijken. Aan het eind heb ik veel gehad aan je verhelderende ideeën over hoe de resultaten te analyseren en wat 'wetenschappelijke juistheid' is. Ik heb ook erg genoten van het kletsen over andere dingen dan de wetenschap op eerdere promotieborrels, niet in de laatste plaats op jouw eigen promotiefeest!

Dr. Van Zanten, beste Bert, bedankt voor het brainstormen over de audiologische kant van de CINGLE-studie en met name de aanvullende experimenten met de CI-patiënten. Ik herin- 
ner me vooral de vele uren die we besteed hebben aan de analyse en interpretatie van de resultaten van de pitchmatch-experimenten, eerst op jouw werkkamer op het Functiecentrum en later (zelfs na je pensioen!) ook bij jou thuis.

Leden van de leescommissie, prof. dr. Bleys, prof. dr. De Boer, prof. dr. Ramsey, prof. dr. Van Dijk, dr. Hol, bedankt voor het kritisch beoordelen van dit proefschrift.

Beste medewerkers van het Audiologisch Centrum en met name het CI-team, bedankt voor het meewerken aan de CINGLE-studie! In het bijzonder wil ik de logopedistes, audiologen en secretaresses bedanken voor hun vele werk! Een extra woord van dank voor Guido is op zijn plaats: bedankt voor de extra metingen en ook voor je geduld en behulpzaamheid.

Beste Niels en Filiep van Cochlear, bedankt voor de jarenlange prettige samenwerking en jullie voortdurende, nauwe betrokkenheid en behulpzaamheid bij ons project.

Beste Sjaak, Digna en Tjeerd, maar ook Huib, Dyan en Ferry, dank voor de prettige kennismaking met wetenschappelijk (dierexperimenteel) onderzoek die jullie me boden in mijn laatste jaar Geneeskunde. Jullie enthousiasme heeft me tijdens die stage, maar ook daarna, enorm gemotiveerd. Ook Inge en Lotty wil ik bedanken voor de interessante projecten die we samen hebben voltooid toen ik nog fulltime onderzoeker was, waarvan er ook twee als hoofdstuk in mijn proefschrift zijn opgenomen. Beste Ivonne, dank voor het mogelijk maken van het onderzoeksblok in het voorjaar van 2020, waarin dit proefschrift voltooid kon worden.

Beste collega arts-assistenten KNO, bedankt voor de gezelligheid in het UMC, maar ook op skireis of op weekend in Brabant. Beste collega-onderzoekers door de jaren heen (op H02, G02, G05 of inmiddels zelfs Q-gebouw), bedankt voor de gezelligheid tijdens de lunch, koffie(half?)uurtje, in de koffie(chocomel?)trein, tijdens bootcamp, tijdens spinning, op summerschool, tijdens congressen en in 2020 ook via Skype-borrels!

Beste Anne en Jan, “mede-CINGLE-aars”, bedankt voor de prettige samenwerking op dit 
project. Anne, al sinds 2015 werken we samen en toen ik eenmaal de kliniek in ging, liet ik de studie met een gerust hart in jouw handen achter. Jan, ook al weer enkele jaren versterk jij ons team, en ook bij jou is de studie in deskundige handen. Ik kijk er naar uit om over enkele jaren ook jullie proefschriften met CINGLE-resultaten te lezen!

Ook veel dank aan alle vrienden die voor de nodige ontspanning tijdens deze lange periode hebben gezorgd. De "dokters" (en alle babies) voor de welkome medische relativering, Kraggenburg en MUST voor alle verhalen over kamperen/vissen en natuurlijk de jaarlijkse decemberactiviteit en -borrel, Complex (ook degenen zonder racefiets ;)) en ook MàD, en natuurlijk mijn Bestuur met dit jaar als hoogtepunt de hilarische ZOOM-pubquizen en een lang weekend Texel zonder kinderen! ;)

Allert en Pronk, mooi dat jullie mij op deze bijzondere dag letterlijk en figuurlijk bijstaan!

Lieve afdeling Schiedam, bedankt voor de ontspanning tijdens weekendjes weg in België/ Duitsland of in Schiedam (of logeren in Utrecht!). Het blijft geweldig om met jullie Scotland Yard of Cards Against Humanity te spelen! En Ten, bedankt voor het prachtige ontwerp voor de omslag van dit proefschrift!

Lieve PiKaKi, of het nou in Utrecht is of in Rosmalen, Maastricht, Heimbach, Reykjavik, Oslo, Balestrand, Helsinki of Edinburgh; als er maar een kelder is, dan komt het wel goed! Lieve Michelle en Sophie, bedankt voor jullie interesse in mijn werk, ook al verschilt het zo enorm van jullie eigen werk. Lieve pap en mam, Pim en Karin, bedankt voor de niet-aflatende interesse en support tijdens de afgelopen jaren. Jullie kunnen het nu nog eens allemaal rustig nalezen. ;)

Lieve Lidwien, met het einde van dit proefschrift "komen we (alweer) in een nieuwe fase"... 
Ik hoop dat er nog vele fases met jou komen! Ik hou van jou.

Lieve Amber en Jolijn, een volgende keer als we een boekje gaan lezen zal ik dit 'boekje' eens uit de kast pakken en jullie vertellen over de behandelingen van eenzijdige doofheid... "En ze leefden nog lang en gelukkig". 


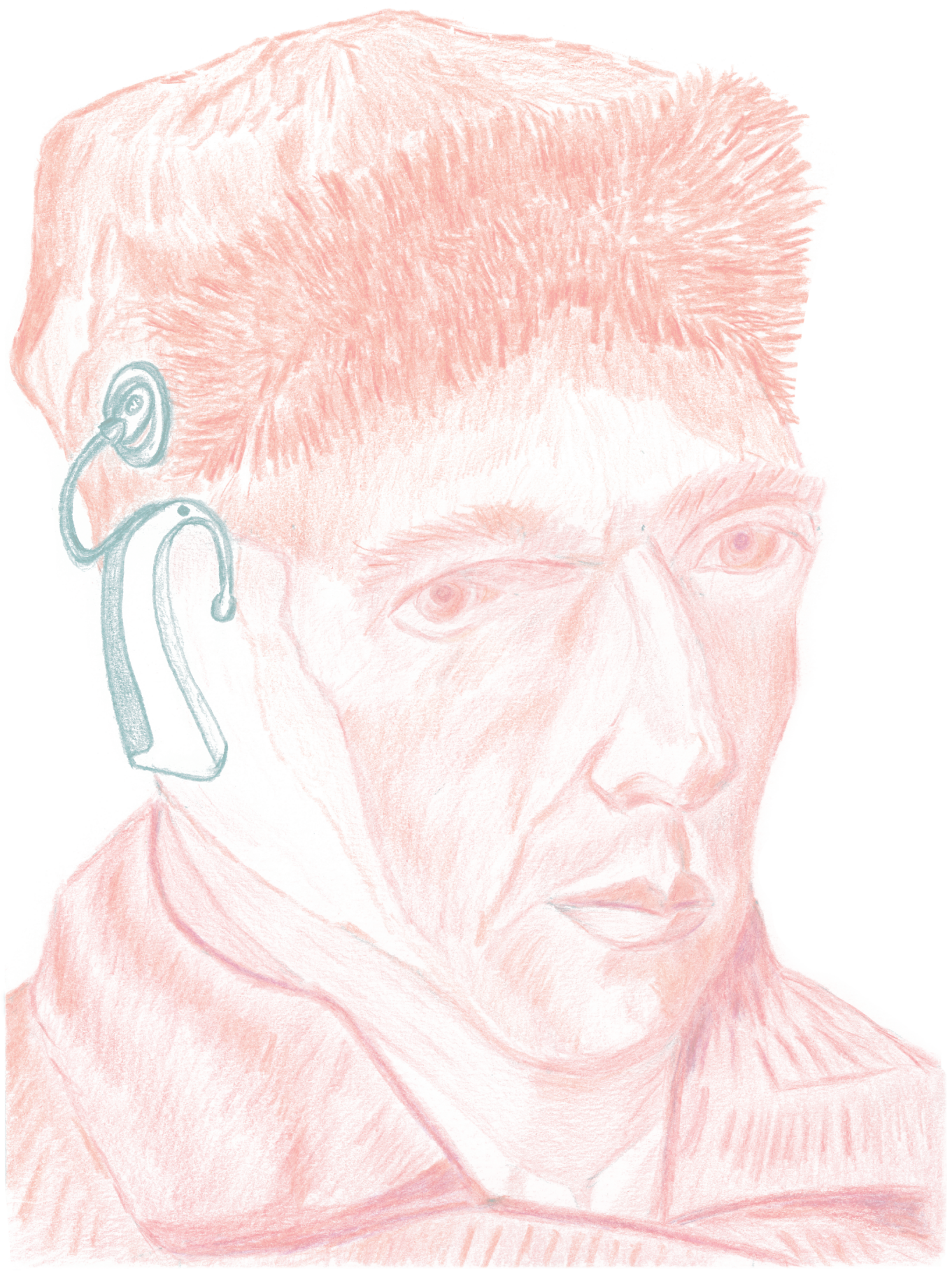




\section{About the Author | Curriculum Vitae}




\section{ABOUT THE AUTHOR | CURRICULUM VITAE}

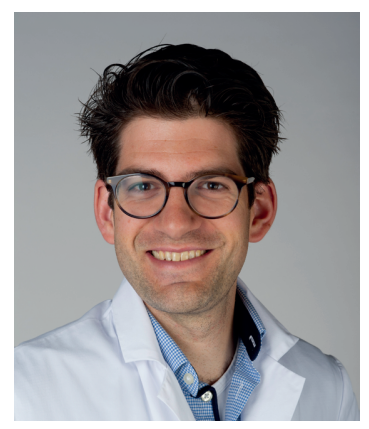

Hieronymus Petrus Maria (Jeroen) Peters was born in Houten, the Netherlands on December $4^{\text {th }}, 1986$. He attended the Elzendaalcollege in Boxmeer and received his gymnasium diploma (cum laude) in 2005.

He moved to Utrecht in August 2005 to start his study Medicine at Utrecht University. After the first year, he graduated for his "propedeuse" (cum laude). He spent two internships abroad: Gynaecology and Obstetrics at the Queen Elizabeth Central Hospital in Blantyre, Malawi and Social Medicine at the Policlínico Belén in Cusco, Peru. He paused his study for one year to devote his time to the fulltime board membership of student's association C.S. Veritas. In his final year of medical school, he did an extra internship Otorhinolaryngology and he completed a research internship at the lab of the Department of Otorhinolaryngology of the UMC Utrecht. After his graduation, he continued working as a researcher in this lab for several months. Meanwhile, he was given the chance to develop a research protocol that ultimately led to this $\mathrm{PhD}$ project under supervision of prof. dr. W. Grolman.

Jeroen officially started his $\mathrm{PhD}$ on January 1st, 2014 focusing on the rehabilitation for single-sided deafness in a randomized controlled trial, of which this thesis is the final product (promotor: prof. dr. R.J. Stokroos, co-promotores: dr. A.L. Smit, dr. G.A. van Zanten). He presented parts of his work at various national and international conferences.

In December 2016, he started his residency at the Department of Otorhinolaryngology and Head \& Neck Surgery of the UMC Utrecht (supervisors: drs. I. Ligtenberg - van der Drift and prof. dr. R.J. Stokroos), and completed parts of his training at Meander Medical Center in Amersfoort (supervisor dr. E.H. van den Akker), St. Antonius Hospital in Nieuwegein (supervisor dr. M.P. Copper), and Gelre Ziekenhuizen in Apeldoorn (supervisor dr. Tj.D. Bruintjes).

He currently lives in the center of Utrecht, together with Lidwien and their daughters Amber (Feb 2018) and Jolijn (Jan 2020). 

This thesis describes the research that was performed by H.P.M. (Jeroen) Peters at the University Medical Center Utrecht in Utrecht, the Netherlands as part of his $\mathrm{PhD}$.

In Part I an introduction on the rehabilitation of single-sided deafness (SSD) is presented. Two literature reviews are described: first, the current treatment options (bone conduction devices and contralateral routing of sound hearing aids) for SSD are discussed. The second review focuses on cochlear implantation for SSD.

Part II describes the study protocol of a randomized controlled trial investigating the treatment options for adult SSD patients. Also, we discuss the short-term results on speech perception in noise, sound localization, tinnitus, and quality of life.

In Part III details additional experiments that were performed in SSD patients implanted with a cochlear implant. They have the unique ability to compare sounds of their cochlear implant to sounds presented to their contralateral normal-hearing ear.

In Part IV, the quality of reporting of systematic reviews and randomized controlled trials in otorhinolaryngology is discussed.

Finally, in Part V, the findings of the previous chapters are summarized and discussed, and future directions for research are provided. 